ANNOUNCEMENT

Assistance Recipients and Non-M\&O/M\&I Contractors

PART I: STI PRODUCT DESCRIPTION

(To be completed by Recipient/Contractor

\section{A. STI Product Identifiers}

1. REPORT/PRODUCT NUMBER(s)

None

2. DOE AWARD/CONTRACT NUMBER(s)

DE-FC36-01ID14209

3. OTHER IDENTIFYING NUMBER(s)

MTU \#010236, E21710

\section{B. Recipient/Contractor}

Michigan Technological University, Houghton, MI

C. STI Product Title

Final Technical Report

\section{Author(s)}

Huang, X.; Hwang, J.Y

E-mail Address(es):

xihuang@mtu.edu; jhwang@mtu.edu

\section{E. STI Product Issue Date/Date of Publication}

03/28/2005

(mm/dd/yyyy)

F. STI Product Type (Select only one)

1. TECHNICAL REPORT

$\triangle$ Final $\square$ Other (specify)

2. CONFERENCE PAPER/PROCEEDINGS

Conference Information (title, location, dates)

\section{JOURNAL ARTICLE}

a. TYPE: $\square$ Announcement Citation Only $\square$ Preprint $\square$ Postprint

b. JOURNAL NAME

c. VOLUME d. ISSUE

e. SERIAL IDENTIFIER (e.g. ISSN or CODEN)

OTHER, SPECIFY

G. STI Product Reporting Period ( $\mathrm{mm} / \mathrm{dd} / \mathrm{yyyy})$

$09 / 24 / 2001$

Thru 12/31/2004
H. Sponsoring DOE Program Office

Golden Field Office

I. Subject Categories (list primary one first)

32: Energy Conservation, Consumption, and Utilization

Keywords

Steelmaking, microwave energy

J. Description/Abstract

Included after second page of form

K. Intellectual Property/Distribution Limitations

(must select at least one; if uncertain contact your Contracting Officer (CO))

$\triangle 1$. UNLIMITED ANNOUNCEMENT (available to

U.S. and non-U.S. public; the Government assumes no liability for disclosure of such data)

2. COPYRIGHTED MATERIAL: Are there any restrictions based on copyright? $\square$ Yes $\square$ No If yes, list the restrictions as retained in your contract

3. PATENTABLE MATERIAL: THERE IS PATENTABLE MATERIAL IN THE DOCUMENT INVENTION DISLOSURE SUBMITTED TO DOE: DOE Docket Number: S(Sections are marked as restricted distribution pursuant to 35 USC 205)

$\square$ 4. PROTECTED DATA: $\square$ CRADA $\square$ Other If other, specify

Release date ( $\mathrm{mm} / \mathrm{dd} /$ yyyy)

5. SMALL BUSINESS INNOVATION RESEARCH (SBIR) DATA

Release date (Required,

(No more than 4 years from date listed in part 1.E above)

6. SMALL BUSINESS TRANSFER (STTR) DATA

Release date (Required,

No more than 4 years from date listed in part 1.E above)

7. OFFICE OF NUCLEAR ENERGY APPLIED TECHNOLOGY

L. Recipient/Contractor Point of Contact Contact for additional information (contact or organization name to be included in published citations and who would receive any external questions about the content of the STI Product or the research contained therein)

Dr. Xiaodi (Scott) Huang, Sr Research Scientist

Name and/or Position

xihuang@mtu.edu 906-487-1822

E-mail

Phone

Michigan Technological University 


\section{PART II: STI PRODUCT MEDIA/FORMAT and LOCATION/TRANSMISSION}

(To be completed by Recipient/Contractor)

\section{A. Media/Format Information:}

1. MEDIUM OF STI PRODUCT IS:

$\triangle$ Electronic Document $\square$ Computer medium

$\square$ Audiovisual material $\square$ Paper $\square$ No full-text

2. SIZE OF STI PRODUCT 172 pages

3. SPECIFY FILE FORMAT OF ELECTRONIC DOCUMENT BEING TRANSMITTED, INDICATE:

$\square$ SGML $\square$ HTML $\square$ XML $\triangle$ PDF Normal $\square$ PDF Image

$\square$ WP-Indicate Version (5.0 or greater)

Platform/operating system

$\square$ MS-Indicate Version (5.0 or greater)

Platform/operating system

$\square$ Postscript

4. IF COMPUTER MEDIUM OR AUDIOVISUAL

a. Quantity/type (specify)

b. Machine compatibility (specify)

c. Other information about product format a user needs to know:

B. Transmission Information:

STI PRODUCT IS BEING TRANSMITTED:

$\square$ 1. Electronic via Elink

$\square$ 2. Via mail or shipment to address indicated in award document (Paper products, $C D-R O M$, diskettes, videocassettes, et.)

\2a. Information product file name (of transmitted electronic format)

14209 Final Technical Report.pdf

\section{PART III: STI PRODUCT REVIEW/RELEASE INFORMATION}

(To be completed by DOE)

\section{A. STI Product Reporting Requirement Review:}

$\square$ 1. THIS DELIVERABLE COMPLETES ALL REQUIRED DELIVERABLES FOR THIS AWARD

$\square$ 2. THIS DELIVERABLE FULFILLS A TECHNICAL REPORTING REQUIREMENT, BUT SHOULD NOT BE DISSEMINATED BEYOND DOE.

\section{B. DOE Releasing Official}

$\square$ 1. I VERIFY THAT ALL NECESSARY REVIEWS HAVE BEEN COMPLETED AS DESCRIBED IN DOE G 241.1-1A, PART II, SECTION 3.0 AND THAT THE STI PRODUCT SHOULD BE RELEASED IN ACCORDANCE WITH THE INTELLECTUAL PROPERTYIDISTRIBUTION LIMITATION ABOVE.

Released by (name)

Date $(\mathrm{mm} / \mathrm{dd} / \mathrm{yyyy})$

E-mail

Phone 


\section{DOE F 241.3 \\ Section J. Description/Abstract}

Steel is a basic material broadly used by perhaps every industry and individual. It is critical to our nation's economy and national security. Unfortunately, the American steel industry is losing competitiveness in the world steel production field. There is an urgent need to develop the next generation of steelmaking technology for the American steel industry.

Direct steelmaking through the combination of microwave, electric arc, and exothermal heating is a revolutionary change from current steelmaking technology. This technology can produce molten steel directly from a shippable agglomerate, consisting of iron oxide fines, powdered coal, and ground limestone. This technology is projected to eliminate many current intermediate steelmaking steps including coking, pellet sintering, blast furnace (BF) ironmaking, and basic oxygen furnace (BOF) steelmaking. This technology has the potential to a) save up to $45 \%$ of the energy consumed by conventional steelmaking; b) dramatically reduce the emission of $\mathrm{CO}_{2}, \mathrm{SO}_{2}$, $\mathrm{NO}_{\mathrm{x}}$, VOCs, fine particulates, and air toxics; c) substantially reduce waste and emission control costs; d) greatly lower capital cost; and e) considerably reduce steel production costs. This technology is based on the unique capability of microwaves to rapidly heat steelmaking raw materials to elevated temperature, then rapidly reduce iron oxides to metal by volumetric heating. Microwave heating, augmented with electric arc and exothermal reactions, is capable of producing molten steel. This technology has the components necessary to establish the "future" domestic steel industry as a technology leader with a strong economically competitive position in world markets.

The project goals were to assess the utilization of a new steelmaking technology for its potential to achieve better overall energy efficiency, minimize pollutants and wastes, lower capital and operating costs, and increase the competitiveness of the U.S. steel industry. The objectives associated with this goal were to a) generate a solid base of technical, marketing, economic, and policy data, b) develop energy, environmental, and economic targets, c) more definitively assess opportunities and barriers, d) accumulate knowledge and experience for defining direction for the next phase of development, and e) promote learning and training of students.

This project consisted of seven tasks:

Task 1 Direct Steelmaking Tests and Technology Assessment

Task 2 Theoretical Flowsheet Development

Task 3 Evaluation of Equipment, Supplies and Worker Environment

Task 4 Evaluation of Steel Company and Supplier Company Interaction and Logistics

Task 5 Energy and Environmental Assessments

Task 6 Marketing and Economic Assessments

Task 7 Evaluation of Policies, Regulations, and Affected Agencies

After three years of successful investigation by Michigan Technological University's multidisciplinary team, with assistance from steel companies, equipment suppliers, an engineering firm, and iron ore mining companies, the following accomplishments have been achieved. 
- Successfully designed, built and continuously modified the world's first bench-scale MW/EAF furnace capable of producing molten steel from iron ore agglomerate in 15-30 minutes

- Successfully conducted a great number of steelmaking tests using this bench-scale MW/EAF furnace and a single mode microwave furnace. The produced steels were of good quality. Operation parameters and relationships among possible influencing factors have been established.

- A series of steelmaking tests were successfully conducted and have proven the technical feasibility, simplicity and extremely low equipment cost on the bench-scale level.

- The potential industry applications of this technology have been better defined based on the experiences and knowledge gained from the project.

- A solution has been found to build large MW/EAF steelmaking systems for industrial operations at low capital cost with present manufacturing technologies and capabilities.

- A general picture of potential changes in worker environment, and steel company and material supplier interaction and logistics has been established.

- A theoretical energy analysis and energy consumption calculation based on the best steelmaking test show a great potential for energy savings with this new steelmaking technology.

- Gas analyses on the off-gas generated during MW/EAF bench-scale steelmaking tests have shown no emission problems. The potential environmental benefits have been estimated.

- The marketing assessment identified a marketing strategy for this technology's commercialization.

- The economic assessment on this technology conduced by an experienced steelmaking engineering firm has shown great reductions in capital cost and operating cost.

- The study of policies, regulations and affected agencies did not find obstacles for implementation of this technology.

- The direction for the next phase of development has been defined.

- Two Ph.D students, two MS students and more than ten undergraduate students have been involved in the project. 
FINAL TECHNICAL REPORT

\title{
Novel Direct Steelmaking By Combining Microwave, Electric Arc, and Exothermal Heating Technologies
}

\author{
DE-FC36-01ID14209 \\ Project Period: September 2001 through December 2004 \\ Submitted: March 31, 2005
}

Principal Investigator: Dr. Xiaodi Huang, 906-487-1822, xihuang@mtu.edu

Co-Investigator: Dr J.Y Hwang, 906-487-2600, jhwang@mtu.edu

\author{
Michigan Technological University \\ Institute of Materials Processing \\ 1400 Townsend Drive \\ Houghton, MI 49931-1295
}

\section{Project Team Members:}

Contributing investigators at Michigan Tech: Dr. B.D. Solomon, Dr. M.C. Roberts, Dr. G.A. Campbell, Dr. Z. Xu, R.C. Greenlund, A.M. Hein, S. Shi, J. Da

Graduate students: Shaolong Qu, PhD; Yongqing Wang, PhD candidate; Laura Devoni, MS; Xiang Sun, MS

External Contributors: Noramco Engineering Corporation, Cleveland-Cliffs Inc., U.P. Steel, Cober Electronics, US Steel, and UP Fabricating Company

Acknowledgment: This report is based upon work supported by the U.S. Department of Energy under Award No. DE-FC36-01ID14209.

Disclaimer: Any findings, opinions, and conclusions or recommendations expressed in this report are those of the author(s) and do not necessarily reflect the views of the Department of Energy. 


\section{TABLE OF CONTENTS}

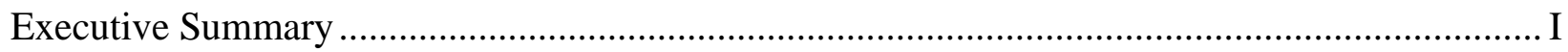

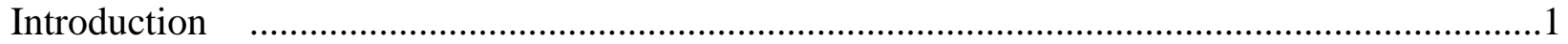

Existing Steel Production Technology................................................................................

The New Steel Production Technology ……………….....................................................2

Project Goals and Objectives .....................................................................................2

Project Work Plan .....................................................................................................

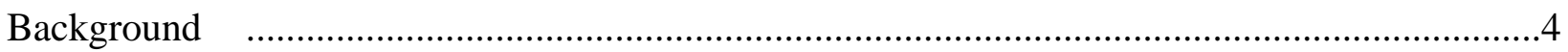

Project Team Members .................................................................................................

Microwave/Material Interactions...................................................................................

Microwave in General...................................................................................

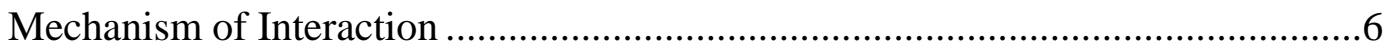

Microwave Adsorption ....................................................................................6

Microwave Processing ...........................................................................................

Microwave Non-Thermal Effect......................................................................

Microwave Processing Systems......................................................................

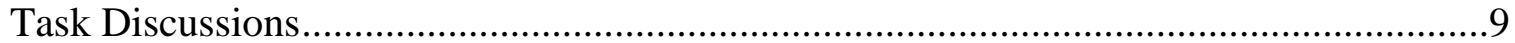

Task 1. Direct Steelmaking Tests and Technology Assessment....................................................10

Baseline Microwave Steelmaking..................................................................................10

Experimental Method.......................................................................................10

Sample Designation ...............................................................................

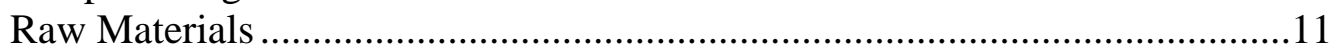

Raw Materials Processing...............................................................................11

Direct Microwave Steelmaking …………...................................................11

Sample Examination ...............................................................................12

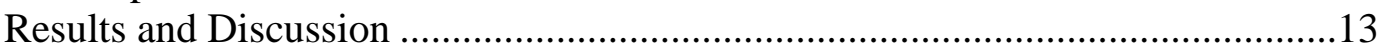

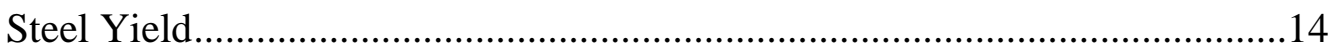

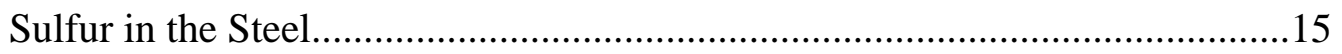

Carbon in the Steel................................................................................15

Phosphorus in the Steel ..................................................................................16

Effects of Iron Ore Types .....................................................................16

Effects of Binder Types ............................................................................16

Pellet Compact Density...................................................................................16

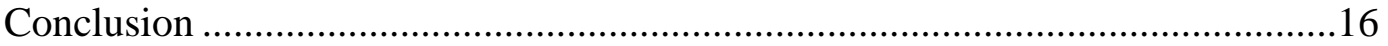

Bench-Scale MW/EAF Steelmaking ..........................................................................18

Microwave Assisted Electric Arc Furnace Design .................................................18

Experimental Method......................................................................................19

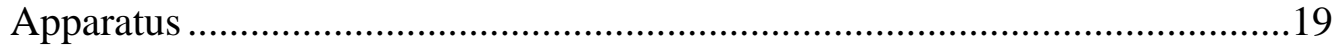

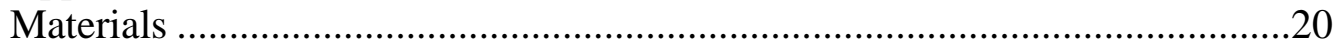

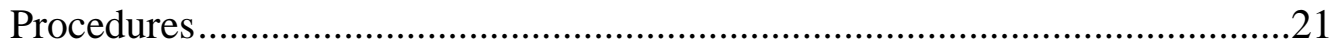


Reduction of Pellet Sample in MW/EAF...............................................21

Coating Crucible Inner Surface ............................................................21

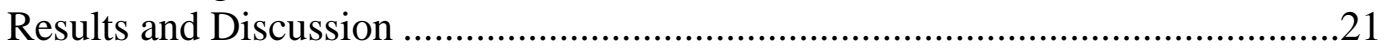

Binders and Lime in Pellet Sample.............................................................22

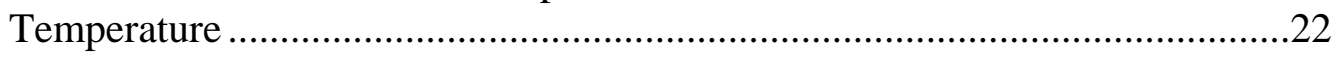

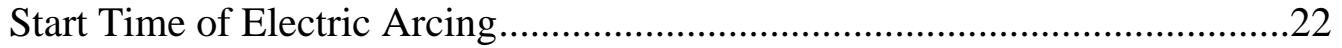

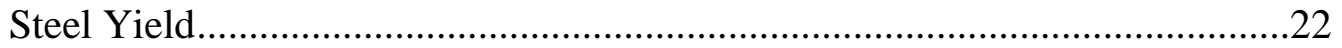

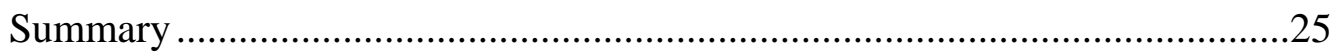

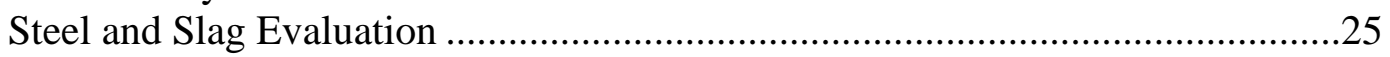

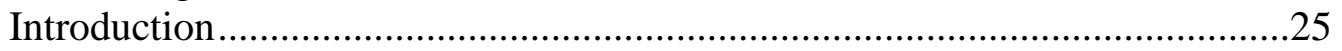

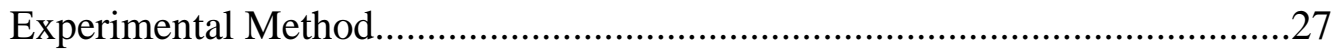

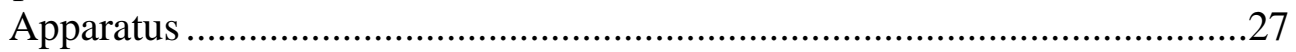

Materials and Procedures.....................................................................27

Steel composition analysis..........................................................22

Sample preparation for XRD study .................................................27

Slag treatment and composition analysis............................................27

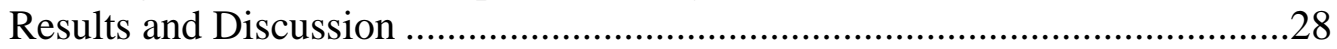

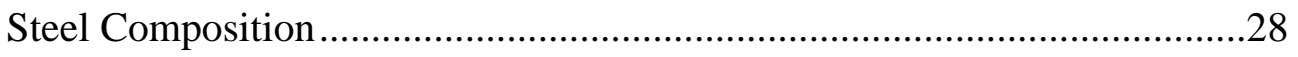

Slag Composition...............................................................................29

Effect of Coal Ratio on Carbon Content of Steel ........................................31

Factors Effecting Sulfur Content of Steel.................................................31

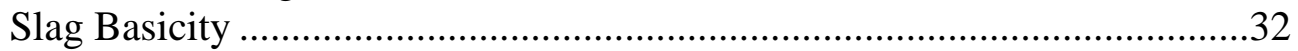

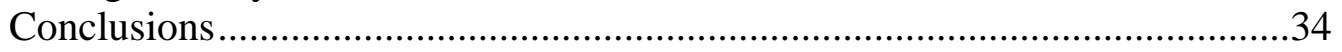

Iron Ore Reduction by Microwave Assisted Hybrid Heating.........................................35

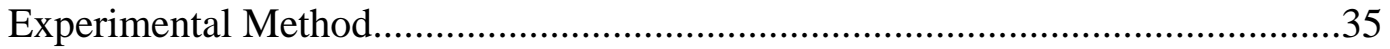

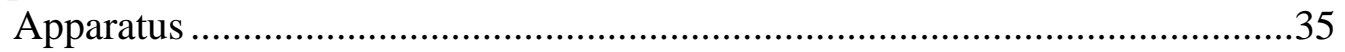

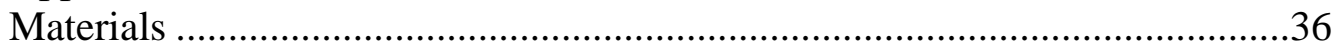

Reducing Agents ..............................................................................36

Experimental Procedure .....................................................................36

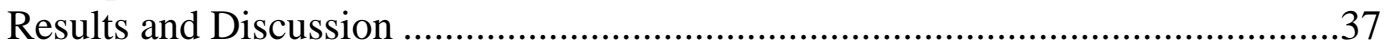

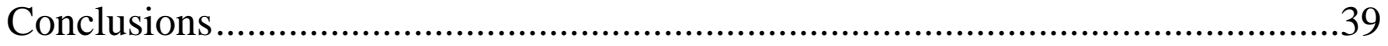

Task 2. Potential Applications and Flowsheet Development ..................................................40

Direct Steelmaking by Batch Operation .....................................................................40

Direct Steelmaking by Continuous Operation ..........................................................41

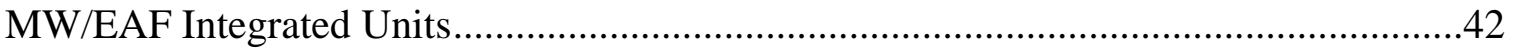

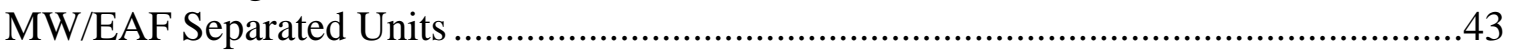

MW Assisted DRI Production (Hybrid Heating) .....................................................44

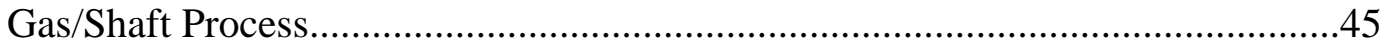

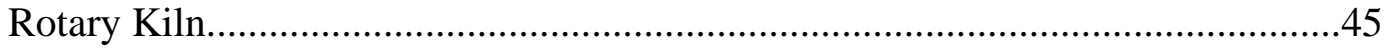

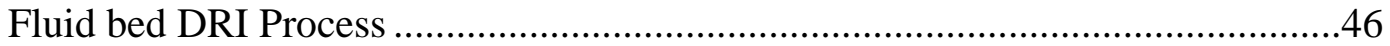

Traveling Grate Process ............................................................................47

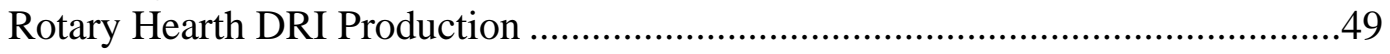

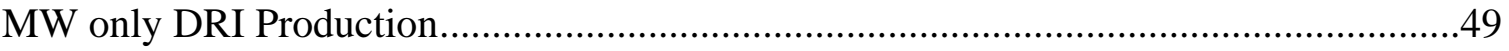


Task 3. Evaluation of Equipment, Supplies, and Worker Environment .....................................51

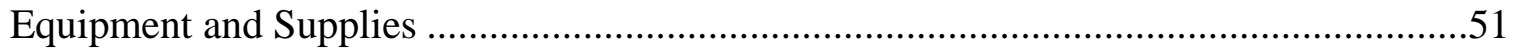

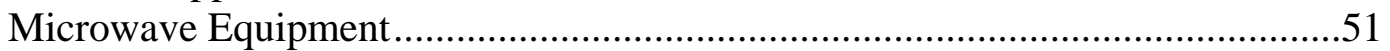

Radio Frequency Equipment..................................................................52

EAF Manufacturers..............................................................................53

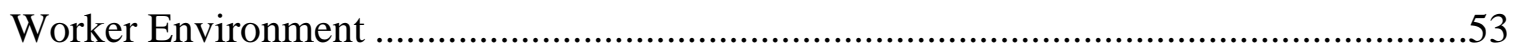

Task 4. Evaluation of Steel Company and Supplier Company Interaction and Logistics .............55

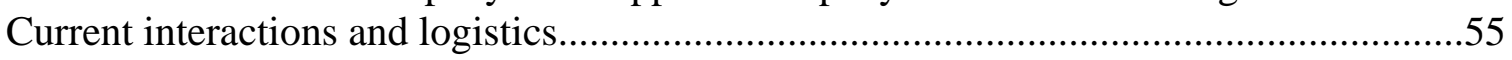

Potential changes to interactions and logistics........................................................58

Scenario 1. Deployment of MW assisted DRI production at mines .....................59

Scenario 2. Deployment of MW assisted DRI production by independent DRI producers ..........................................................59

Scenario 3. Deployment of MW assisted DRI production or direct steelmaking at mini mills ................................................................................60

Conclusion

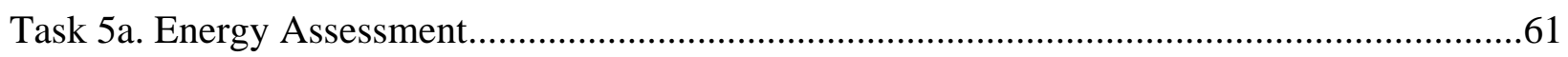

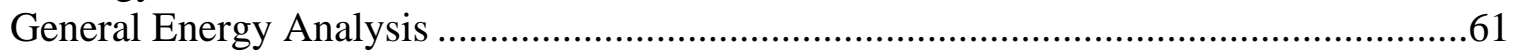

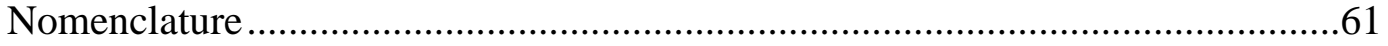

Temperature Change in MW/EAF Process....................................................62

Thermodynamic Analysis of Chemical Reactions..............................................63

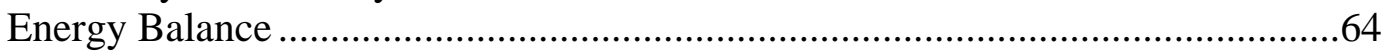

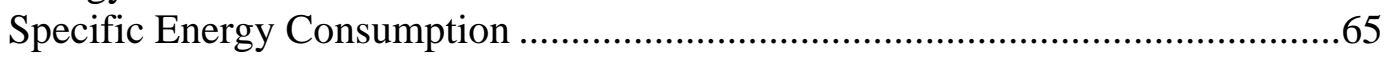

Minimum Coal to Magnetite Ratio for Complete Reduction ..............................66

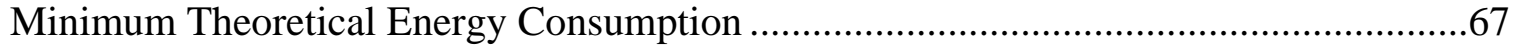

Basic Assumptions and Equations ..............................................................67

Minimum Theoretical Energy Consumption Formulas ....................................68

Minimum Theoretical Energy Consumption Computation ................................71

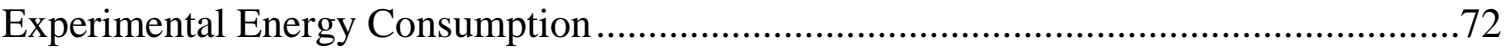

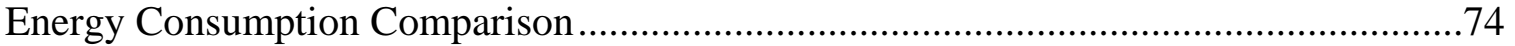

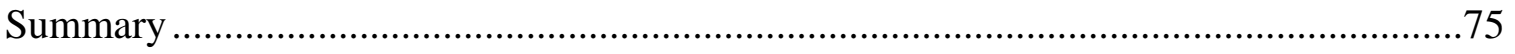

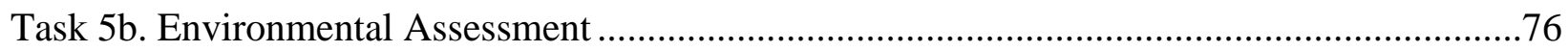

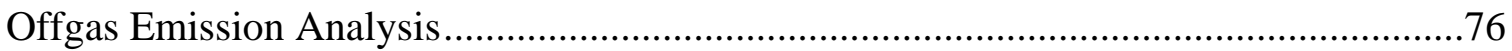

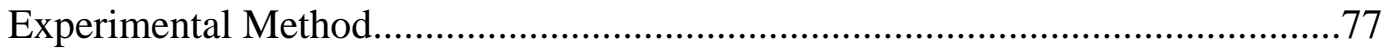

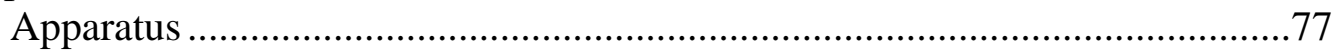

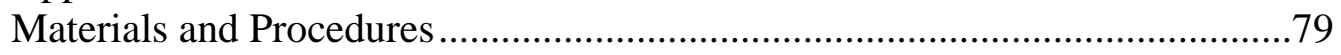

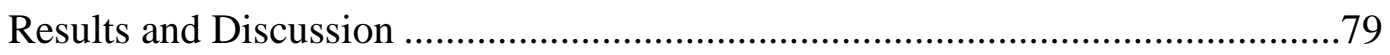

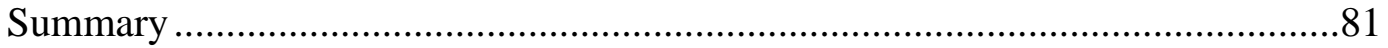

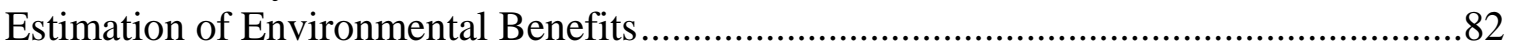

Task 6. Marketing and Economic Assessments...................................................................84

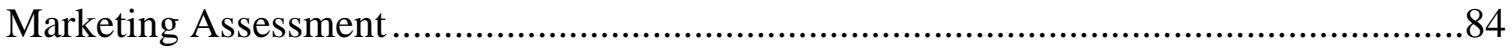

Current steel-making and iron-making technologies ........................................84

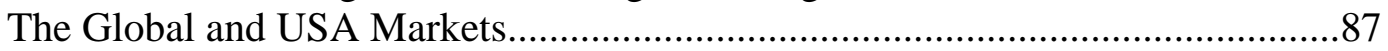


Use of Microwave Technology to Improve Competitiveness .............................89

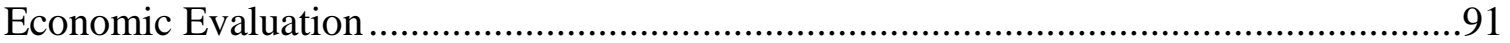

Economic Feasibility of a 500,000 TPY Plant..............................................................91

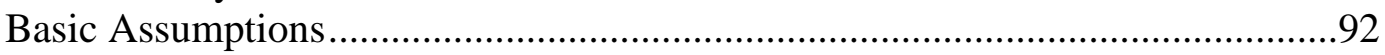

Applications to be analyzed ..................................................................92

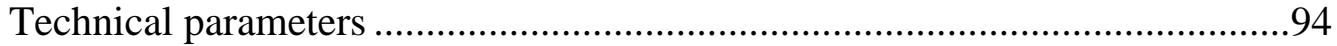

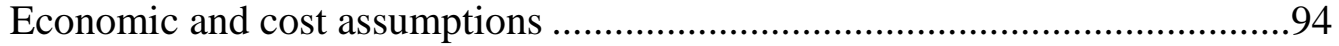

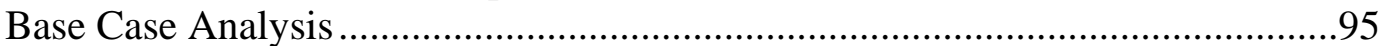

Sensitivity Analysis .............................................................................96

Task 7. Evaluation of Policies, Regulations, and Affected Agencies......................................97

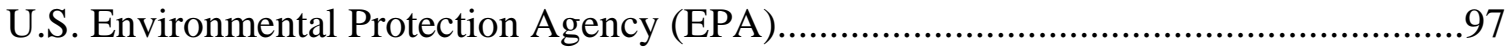

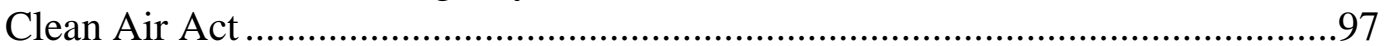

Clean Water Act..................................................................................................100

Resource Conservation and Recovery Act .....................................................101

Emergency Planning and Community Right-to-Know Act ...............................102

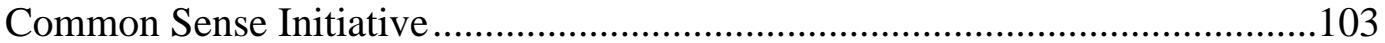

Occupational Safety and Health Administration (OSHA) ..........................................104

U.S. Department of Transportation (DOT) .................................................................104

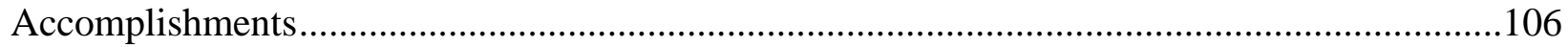

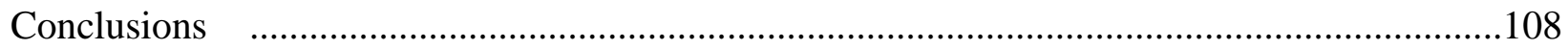

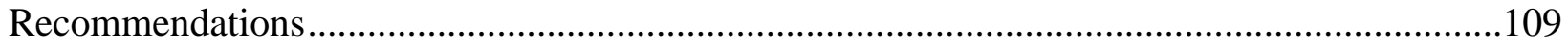

References

\section{LIST OF APPENDICES}

Appendix A: Baseline microwave steelmaking test parameters and results................................1

Appendix B: Summary of MW/EAF Steelmaking Results ...............................................26

Appendix C: Composition of Offgases in the MW/EAF Process............................................29

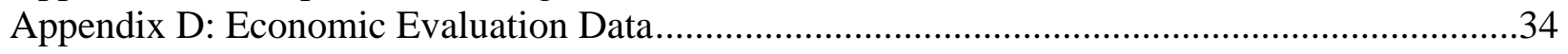




\section{LIST OF FIGURES}

1. Current Steelmaking Process

2. New Microwave/EAF Steelmaking Technology …….....................................................2

3. Mechanism of interaction between microwave and materials ..................................................6

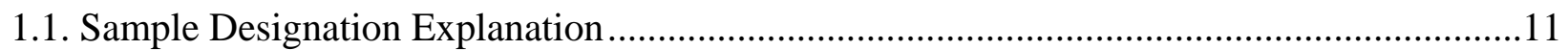

1.2. Wavemat MCR200 Microwave Furnace ..........................................................................12

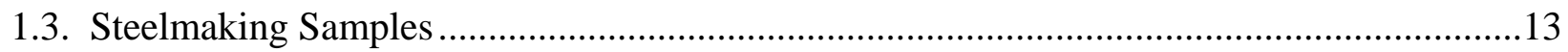

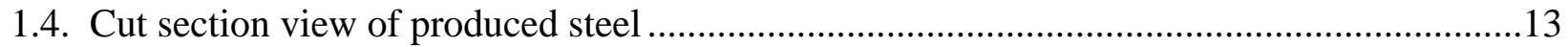

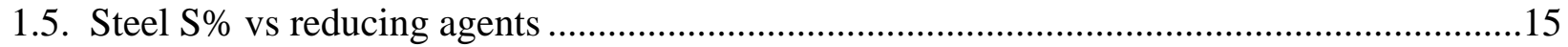

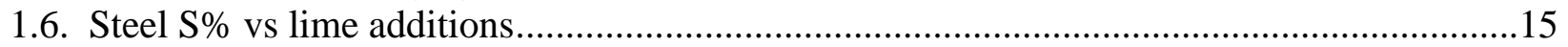

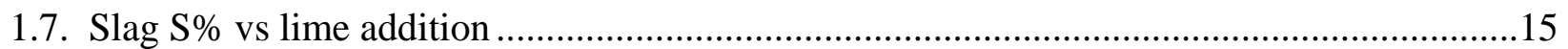

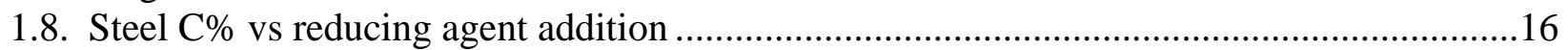

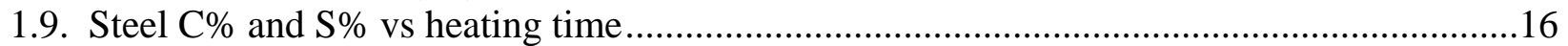

1.10 Schematic diagram of microwave assisted electric arc furnace............................................18

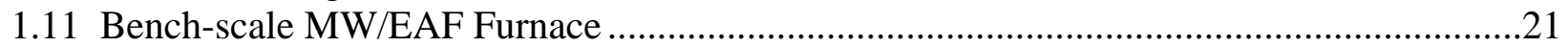

1.12 Technician loading fireclay crucible into furnace chamber...................................................21

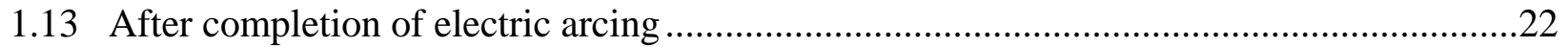

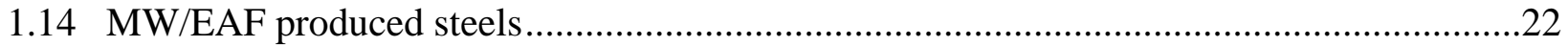

1.15 Effects of electric arcing time and composition on steel yield ............................................23

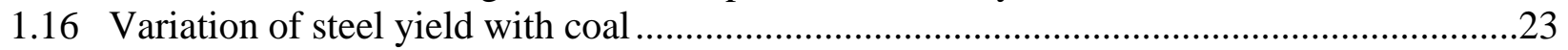

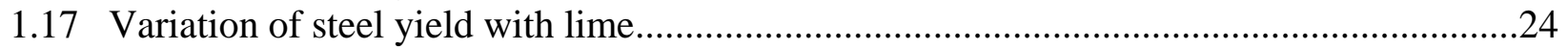

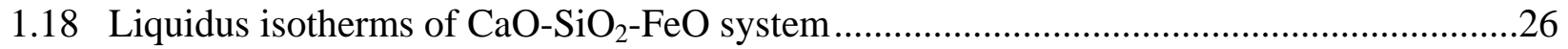

1.19 XRD spectrums of low steel yield slag..............................................................................30

1.20 Variation of carbon content with pellet coal ratio ............................................................31

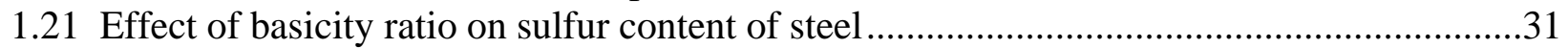

1.22 Effects of crucible and lining on sulfur content of steel .................................................32

1.23 Variation of slag basicity ratio with lime ratio of pellet sample with fireclay crucible.........32

1.24 Basicity ratio of slag generated in fireclay crucible with lining ..........................................33

1.25 Basicity ratios of slag generated in magnesia crucible with or without lining .......................33

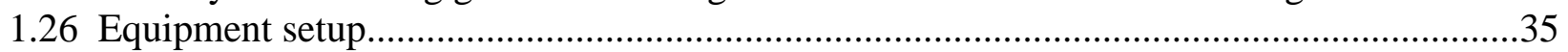

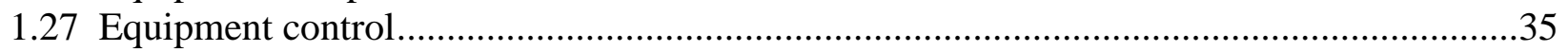

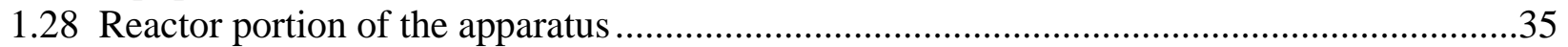

1.29 Schematic diagram of hybrid heating apparatus ................................................................36

1.30 Comparison of conventional and microwave assisted magnetite reduction .........................37

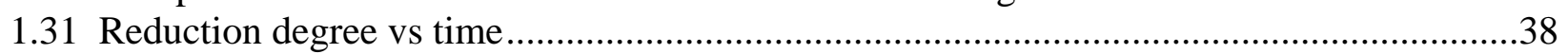

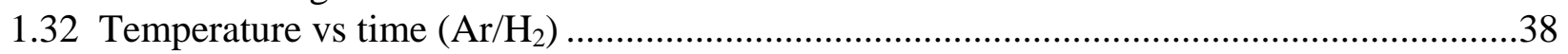

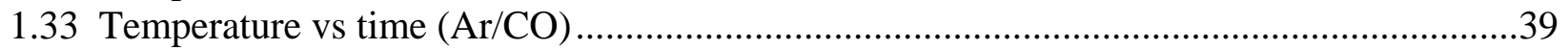

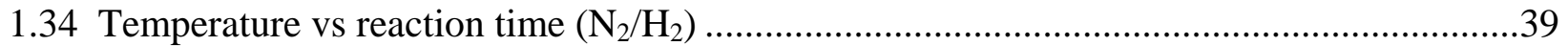

2.1. Batch Operation Microwave Electric Arc Furnace.............................................................41

2.2. Continuous Operation Microwave Electric Arc Furnace..........................................................4

2.3. Flowsheet Comparison of conventional versus MW/EAF steelmaking ..................................42

2.4. Flowsheet for steelmaking using separated MW/EAF units.............................................43 
2.5. Conceptual design of MW/EAF unit based on $1 \mathrm{~kW}$ MW generators .................................44

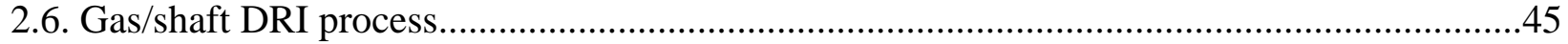

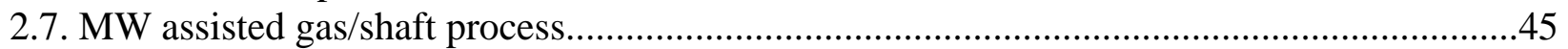

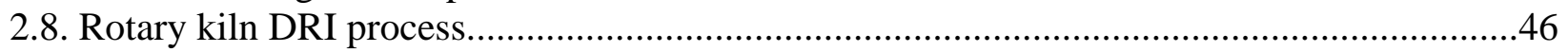

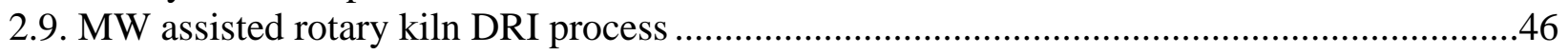

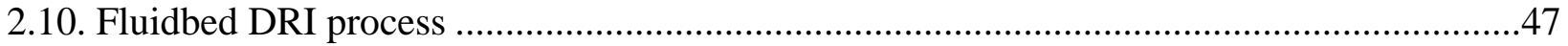

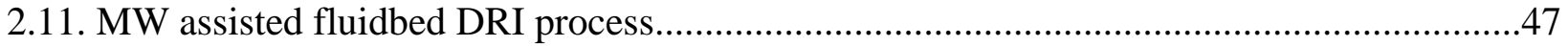

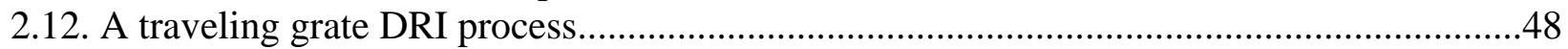

2.13. MW assisted traveling grate DRI process..................................................................48

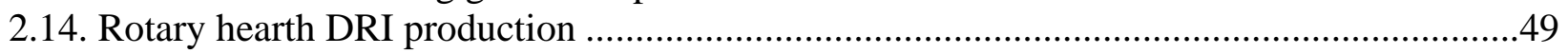

2.15. MW DRI reducing furnace and gaseous fuel generator.................................................50

3.1. Industrial Microwave Heating System.........................................................................51

3.2. Microwave system to calcine refractory gold ores and concentrates .................................51

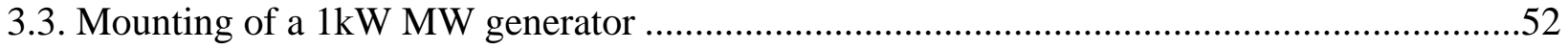

3.4. MW assisted furnace using multiple 1kW MW generators ...........................................52

3.5. Industries and processes that utilize radio frequency heating ...........................................53

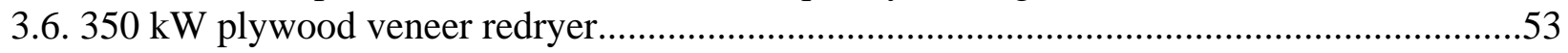

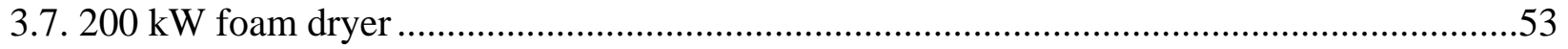

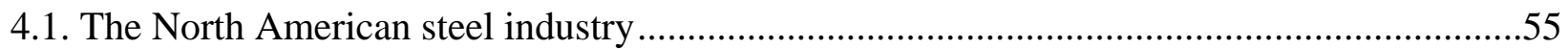

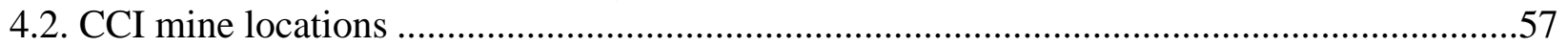

5.1. Variation of burden temperature in MW/EAF process .................................................62

5.2. Photograph of constituent parts in offgas analysis system ...........................................78

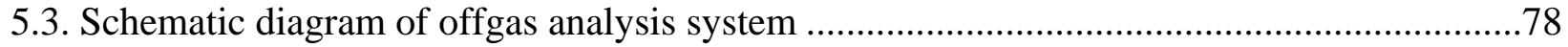

6.1. Material Flows in Microwave Steel Making ...............................................................93 


\section{LIST OF TABLES}

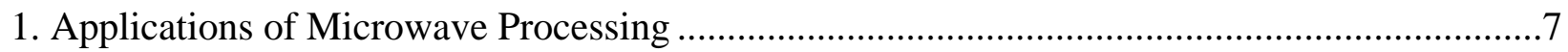

1.1. Composition of the ores (\%) ........................................................................................11

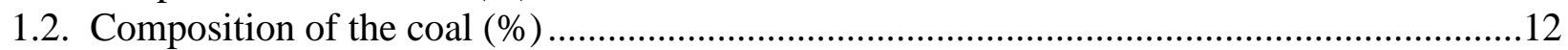

1.3. Composition of Starch, Bentonite, Graphite, and Lime (\%)..........................................12

1.4. Pellet compositions and steel yield ..................................................................................

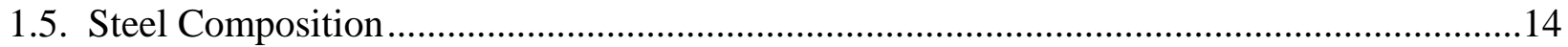

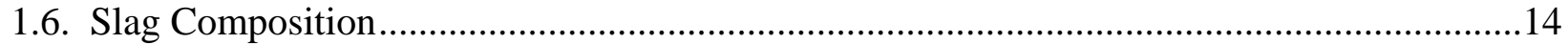

1.7. Pellet composition by weight ratio ...........................................................................20

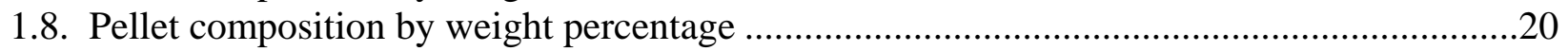

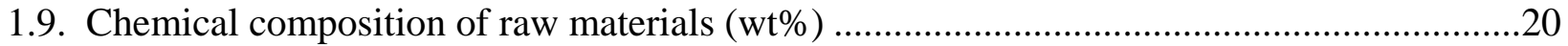

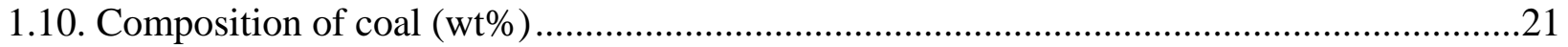

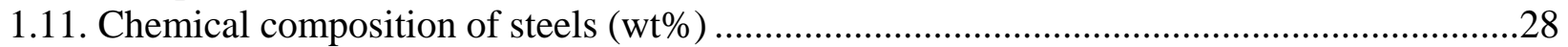

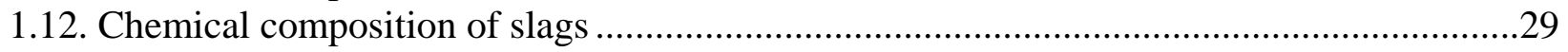

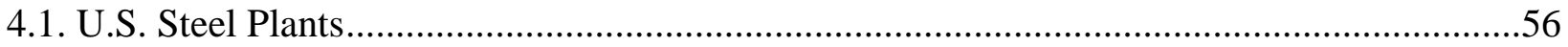

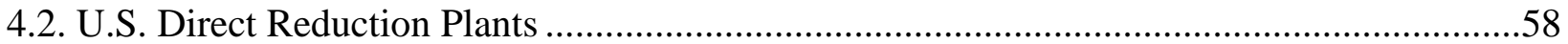

5.1. Minimum ratios of different coals to magnetite ore with $81.2 \%$ magnetite .............................66

5.2. Minimum theoretical energy consumption using high volatile coal.......................................71

5.3. Minimum theoretical energy consumption using low volatile coal........................................72

5.4. Experimental results and compositions of pellet sample and raw materials ..........................73

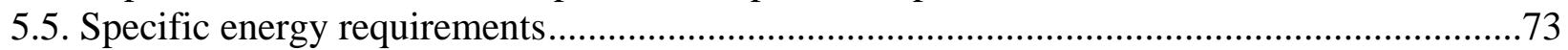

5.6. Specific energy consumption comparisons...........................................................................

5.7. Average composition of offgas from the MW/EAF process ………………...........................8

5.8. Estimated intensities of combustion-related pollutants from the MW/EAF process...............80

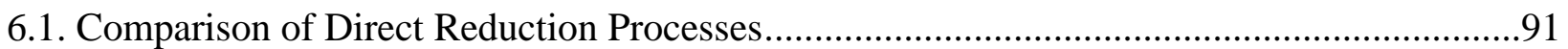

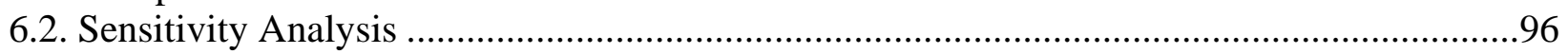




\section{EXECUTIVE SUMMARY}

Steel is a basic material broadly used by perhaps every industry and individual. It is critical to our nation's economy and national security. Unfortunately, the American steel industry is losing competitiveness in the world steel production field. There is an urgent need to develop the next generation of steelmaking technology for the American steel industry.

Direct steelmaking through the combination of microwave, electric arc, and exothermal heating is a revolutionary change from current steelmaking technology. This technology can produce molten steel directly from a shippable agglomerate, consisting of iron oxide fines, powdered coal, and ground limestone. This technology is projected to eliminate many current intermediate steelmaking steps including coking, pellet sintering, blast furnace (BF) ironmaking, and basic oxygen furnace (BOF) steelmaking. This technology has the potential to a) save up to $45 \%$ of the energy consumed by conventional steelmaking; b) dramatically reduce the emission of $\mathrm{CO}_{2}, \mathrm{SO}_{2}$, $\mathrm{NO}_{x}$, VOCs, fine particulates, and air toxics; c) substantially reduce waste and emission control costs; d) greatly lower capital cost; and e) considerably reduce steel production costs. This technology is based on the unique capability of microwaves to rapidly heat steelmaking raw materials to elevated temperature, then rapidly reduce iron oxides to metal by volumetric heating. Microwave heating, augmented with electric arc and exothermal reactions, is capable of producing molten steel. This technology has the components necessary to establish the "future" domestic steel industry as a technology leader with a strong economically competitive position in world markets.

The project goals were to assess the utilization of a new steelmaking technology for its potential to achieve better overall energy efficiency, minimize pollutants and wastes, lower capital and operating costs, and increase the competitiveness of the U.S. steel industry. The objectives associated with this goal were to a) generate a solid base of technical, marketing, economic, and policy data, b) develop energy, environmental, and economic targets, c) more definitively assess opportunities and barriers, d) accumulate knowledge and experience for defining direction for the next phase of development, and e) promote learning and training of students.

This project consisted of seven tasks:

Task 1 Direct Steelmaking Tests and Technology Assessment

Task 2 Theoretical Flowsheet Development

Task 3 Evaluation of Equipment, Supplies and Worker Environment

Task 4 Evaluation of Steel Company and Supplier Company Interaction and Logistics

Task 5 Energy and Environmental Assessments

Task 6 Marketing and Economic Assessments

Task 7 Evaluation of Policies, Regulations, and Affected Agencies

After three years of successful investigation by Michigan Technological University's multidisciplinary team, with assistance from steel companies, equipment suppliers, an engineering firm, and iron ore mining companies, the following accomplishments have been achieved. 
- Successfully designed, built and continuously modified the world's first bench-scale MW/EAF furnace capable of producing molten steel from iron ore agglomerate in 15-30 minutes

- Successfully conducted a great number of steelmaking tests using this bench-scale MW/EAF furnace and a single mode microwave furnace. The produced steels were of good quality. Operation parameters and relationships among possible influencing factors have been established.

- A series of steelmaking tests were successfully conducted and have proven the technical feasibility, simplicity and extremely low equipment cost on the bench-scale level.

- The potential industry applications of this technology have been better defined based on the experiences and knowledge gained from the project.

- A solution has been found to build large MW/EAF steelmaking systems for industrial operations at low capital cost with present manufacturing technologies and capabilities.

- A general picture of potential changes in worker environment, and steel company and material supplier interaction and logistics has been established.

- A theoretical energy analysis and energy consumption calculation based on the best steelmaking test show a great potential for energy savings with this new steelmaking technology.

- Gas analyses on the off-gas generated during MW/EAF bench-scale steelmaking tests have shown no emission problems. The potential environmental benefits have been estimated.

- The marketing assessment identified a marketing strategy for this technology's commercialization.

- The economic assessment on this technology conduced by an experienced steelmaking engineering firm has shown great reductions in capital cost and operating cost.

- The study of policies, regulations and affected agencies did not find obstacles for implementation of this technology.

- The direction for the next phase of development has been defined.

- Two Ph.D students, two MS students and more than ten undergraduate students have been involved in the project. 


\section{INTRODUCTION}

The American steel industry is vital to our economy and national security. Unfortunately, the industry is losing competitiveness in the world steel production. In the last several years, more than 30 steel companies have claimed bankruptcy, including long time giants such as LTV, Bethlehem, National, Weirton, Rouge, etc. Domestic steel production has shrunk from 130 million tons in 1976 to 90 million tons in 2003, while world steel production increased from 533 million tons in 1976 to 960 million tons in 2003. Currently, the U.S. is one of the largest importers of steel in the world, importing about 30 million tons per year. Our steel industry is no longer competitive in the world market. The shortage of steel and the recent skyrocketing prices have left many industries that utilize steel in desperation.

The U.S. government and the industry have realized the need to develop the next generation steel production technology. In 2001, the U.S. Department of Energy issued a RFP to look for the technology which would meet its technical feasibility, energy efficiency, cost savings, environmental goals, and eliminate the use of coke. Novel direct steelmaking by combining microwave, electric arc and exothermal heating technologies was selected as one of candidates.

The steel produced in the U.S. comes from two types of operations: integrated mills and minimills. Integrated mills utilize a blast furnace (BF) to produce liquid iron from iron ore and then remove excessive carbon in liquid iron in a basic oxygen furnace (BOF) to produce high quality but expensive steel. Minimills employ electric arc furnaces (EAF) to melt steel scrap with DRI (Direct Reduced Iron) and produce generally lower quality but lower cost steel. The aging domestic integrated steel mills' processes require the use of expensive, polluting coke, and will require huge investments to update their equipment. Integrated mills are losing market share to the minimills, and account for about $50 \%$ of the 90 million tons of domestic production.

The primary technical barrier for the U.S. steel industry is how to reduce iron oxide to iron with coal instead of coke or reformed gas in an efficient, economical and environmentally friendly way. Environmental regulations and high cost have caused the shut down of more than half of the domestic coke batteries (from 179 in 1979 to 78 in 1996), forcing the use of expensive imported coke (coke price has increased from $\$ 80$ to $\$ 300-400 /$ ton). Minimills traditionally enjoyed an abundant supply of domestic steel scrap; however, the recent strong demand for scrap internationally has doubled the price. DRI prices have also significantly increased due to high cost reformed natural gas, causing many DRI plant closings. The U. S. Steel industry needs breakthrough technology to overcome these critical barriers.

\section{Existing Steel Production Technology}

Blast furnace technology for iron production has been employed for approximately 150 years. Many incremental improvements have been made and it is the current dominant technology. As shown in Figure 1, fired iron ore pellets, coke and lime are charged into a blast furnace. Air is blown in at high speed to combust the coke to generate carbon monoxide and heat. Fired iron ore pellets are reduced to iron hot metal by the carbon monoxide and melted to form liquid iron. The liquid iron is then sent to a BOF where pure oxygen is blown into the liquid iron to remove the carbon and convert iron into steel (Figure 1). The fundamental problems associated with this 
steel production route are the needs for coke and intensified combustion. Coke making generates many pollutants and consumes large volume of water for coke quenching. Intensified combustion generates great amounts of dust and wastes energy in the form of exhaust gases. Treating hot dust needs large volumes of water and produces pollutants.

\section{The New Steel Production Technology}

The new microwave assisted EAF

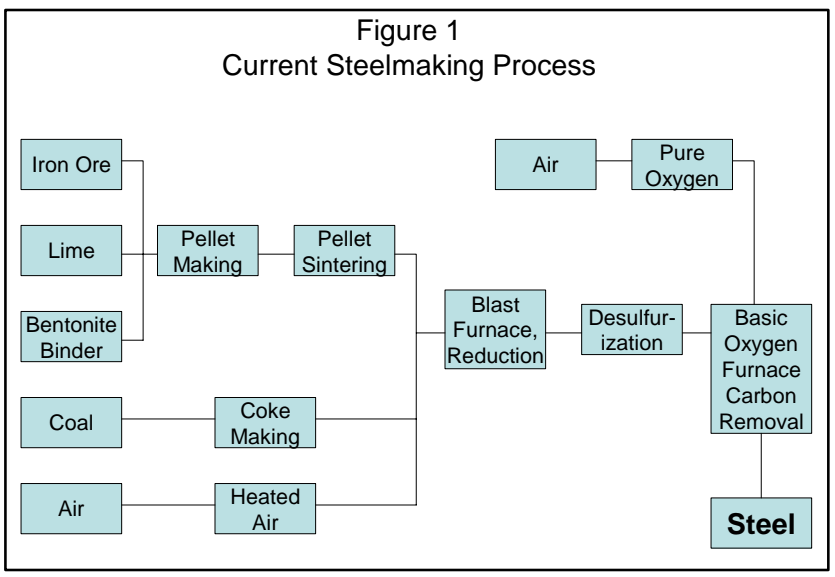
steelmaking technology is a revolutionary change from current technology. It is achieved through the combination of microwave, electric arc and exothermal heating. This technology can produce molten steel directly from a shippable agglomerate (green ball) consisting of iron oxide concentrate, coal, and fluxing agent without the intermediate steps of coking, sintering, BF ironmaking, and BOF steelmaking. The unique aspect of this technology utilizes the advantages of rapid volumetric heating, high energy efficiency, and chemical reaction acceleration through the use of microwaves. The viability of the technology lies in that iron ore and carbon are excellent microwave absorbers. This concept utilizes the combination of microwaves, electric arc, and exothermal reaction heating to provide the steelmaking energy. This new, simplified process translates into less capital cost, higher productivity, less environmental pollution and treatment cost, higher energy efficiency, and lower production cost. In addition, the concept remains flexible in that it can still produce molten steel from a scrap charge, thus allowing different feed stocks to be run through the same furnace.

In this new process, iron ore is crushed, ground, and concentrated by conventional processing. The concentrated iron oxide is mixed with pulverized coal and limestone, and then agglomerated at ambient temperature to provide strength for handling. Coal serves as a reducing agent for iron oxides and as an auxiliary heat source via an exothermal oxidation reaction. Limestone is used as the fluxing agent (Figure 2).

\section{Project Goals and Objectives}

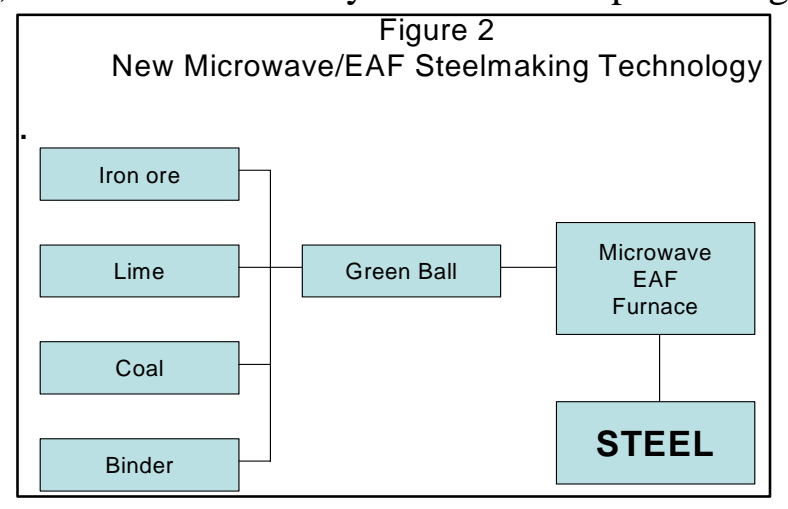

The goal of this project was to assess the utilization of a new steelmaking technology that has the potential to achieve better overall energy efficiency, minimize pollutants and wastes, lower capital and operating costs, and increase the competitiveness of the U.S. steel industry. The objectives associated with this goal were to a) generate a solid base of technical, marketing, economic, and policy data, b) develop energy, environmental, and economic targets, c) more definitively assess opportunities and barriers, d) accumulate knowledge and experience for 
defining direction for the next phase of development, and e) promote learning and training of students.

\section{Project Work Plan}

Michigan Technological University (MTU), as the primary project contractor, provided a multidisciplinary team of engineers, scientists, faculty, technicians and students with expertise in metallurgical engineering, chemical engineering, and business and economics. This project also obtained assistances from steel companies, equipment suppliers, an engineering firm, and iron ore mining companies.

This project consisted of seven tasks as listed below.

Task 1 Direct Steelmaking Tests and Technology Assessment

Task 2 Theoretical Flowsheet Development

Task 3 Evaluation of Equipment, Supplies and Worker Environment

Task 4 Evaluation of Steel Company and Supplier Company Interaction and Logistics

Task 5 Energy and Environmental Assessments

Task 6 Marketing and Economic Assessments

Task 7 Evaluation of Policies, Regulations, and Affected Agencies 


\section{BACKGROUND}

Information about the project team members and more details about microwave processing are provided in this section.

\section{Project Team Members}

The Institute of Materials Processing at Michigan Tech initiated the microwave steelmaking research using a single mode microwave sintering system in 1995. A working model combining microwave, electric arc, and exothermal heating was identified. Molten steel has been produced in the furnace in a few minutes. The early research was supported by the Institute's internal research dollars. In 2001, Michigan Tech was awarded a grant by the DOE to conduct bench scale research utilizing the new microwave steelmaking invention. This research included technology assessment, flowsheet development, evaluation of equipment, evaluation of logistics, energy and environmental assessments, marketing and economic assessments, and evaluation of policies and regulations. A pilot plant scale up is the next logical phase.

U.P. Steel is a private company created in 2001. The founders of the company have extensive experience in mining, mineral processing and steelmaking, and identified microwave steelmaking as the most important and promising technology for the future of the industry. U.P. Steel licensed this technology for commercialization. U.P Steel has filed world wide patent applications and extensively marketed the process with $\$ 100,000$ of current investment.

Cleveland-Cliffs Inc. is the largest domestic iron ore company. CCI participated in Michigan Tech's DOE microwave steelmaking project by providing iron ore for steelmaking tests and assistance in evaluating test results and economics. CCI has closely watched the progress of the microwave steelmaking technology since 2001 and is actively involved in the proposed pilot plant planning and cost sharing.

U.P. Fabricating is a leading steel fabricating company in the Upper Peninsula of Michigan. This company has a long history of working with Michigan Tech in developing new mineral processing equipment. U.P. Fabricating has participated in the Michigan Tech's DOE microwave steelmaking project by providing more than 20 magnetrons, a tiltable steel vessel and various fabrication assistances.

Noramco Engineering is a major engineering firm serving the iron and steel companies, and has been working with both Michigan Tech and U.P. Steel to evaluate the economics of the new steelmaking technology and scale up design. This company estimated that the capital per annual ton is $\$ 48$ for microwave steelmaking in comparison with $\$ 1750$ for traditional steelmaking and the operating cost per ton is $\$ 68$ for microwave steelmaking in comparison with $\$ 120$ for traditional steelmaking. Based on their experience and knowledge in steelmaking equipment, Noramco has completed a scale up design which can produce 500,000 to 1,000,000 tons of steel per year. Noramco's involvement to date has been self funded. 


\section{Microwave/Material Interactions}

\section{Microwave in General}

Microwaves have extensive application in the field of communication, but certain frequencies are allocated for industrial and scientific application. Microwaves are electromagnetic waves with frequencies that range from 0.3 to $300 \mathrm{GHz}$. Microwave frequencies include three bands: the ultrhigh frequency (UHF: $0.3 \mathrm{GHz}$ to $3 \mathrm{GHz}$ ), the superhigh frequency (SHF: $3 \mathrm{GHz}$ to 30 $\mathrm{GHz}$ ) and the extremely high frequency (EHF: $30 \mathrm{GHz}$ to $300 \mathrm{GHz}$ ). Microwaves obey the laws of optics and can be transmitted, absorbed or reflected. The behavior of microwave depends on the type of materials interacted with.

Microwaves are generated from magnetrons. Microwave energy is converted from electric energy and the frequency of the generated microwave is a dominant parameter of the conversion efficiency. The frequency of a generated microwave depends on the design of the circuit associated with magnetrons. The microwave energy generated by a magnetron is dependent on the line voltage applied on the magnetron and on the amount of time the magnetron has been operated.

The basic power equation for a microwave oven is stated as follows:

$$
P_{G}=P_{R}+P_{A}+P_{D}
$$

The power generated by a magnetron, $\mathrm{P}_{\mathrm{G}}$, equals the power reflected back to the magnetron, $\mathrm{P}_{\mathrm{R}}$, plus the power absorbed by the microwave oven cavity and feed system, $\mathrm{P}_{\mathrm{A}}$, plus the power delivered to the load inside the microwave oven, $\mathrm{P}_{\mathrm{D}}$.

\section{Behavior of Materials under Microwave Irradiation}

Microwaves can be reflected, absorbed and transmitted by materials with which they interact. Reflection and absorption are due to the interaction of atoms inside materials with the electric field of microwave. Transmission is the result of partial reflection and incomplete absorption.

Materials reflect and absorb microwave energy to various degrees depending on their composition, structure, temperature and the frequency of the microwave. With regard to their response to microwaves, materials are divided into three categories: transmitters, absorbers or reflectors. Metals and graphite, at least in bulk form, are excellent reflectors and do not absorb microwaves at all. Some minerals (magnetite, iron titanite, galena et al.) are excellent absorbers.

The absorption degree of some materials can be changed through various methods, which include changing the temperature, adding absorbing constituents, altering the microstructure and defect structure of materials, changing the form of materials (e.g. bulk vs. powder), and changing the frequency of the incident microwave. At room temperature, many ceramics and polymers do not appreciably absorb microwaves with a frequency of $2.45 \mathrm{GHz}$, however, their absorption can be increased by increasing the temperature of processing. 


\section{Mechanism of Interaction}

Microwaves can interact with materials through either dielectric polarization or ionic conduction, as illustrated by Figure 3. Dielectric polarization involves the short-range displacement of a charge through the formation and rotation of electric dipoles. Ionic conduction involves the longrange transport of charge.

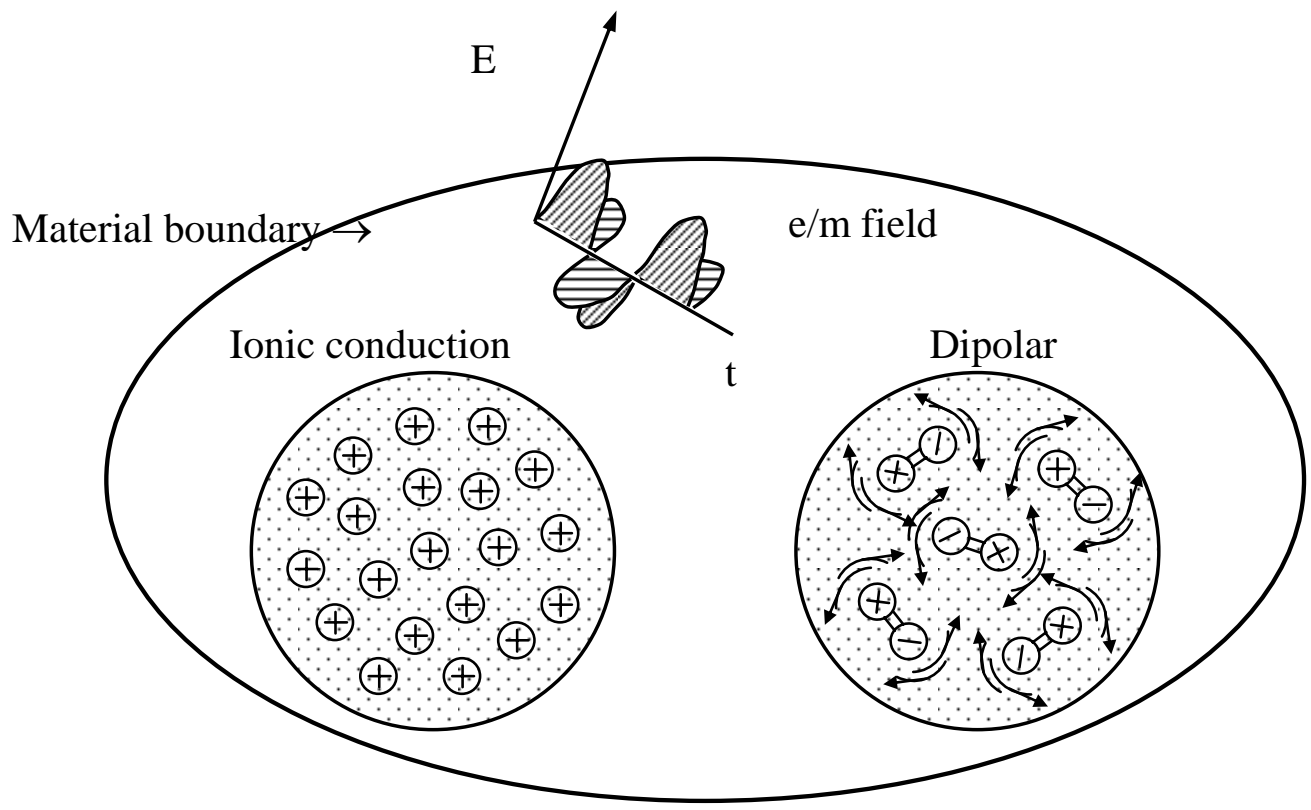

Figure 3. Mechanism of interaction between microwave and materials

Both processes result in the absorption of microwave energy by materials. Microwave energy loss through ionic conduction is due to the well-known ohmic losses, which occur when ions move through the materials and collide with other species. The time allowed for an ion's transport in the direction of the field decreases with increasing frequency of microwave, so ionic conduction is the dominant process at low frequencies. In a microwave region, dipoles are formed and rotate to align themselves in phase with the reversing electric field. The extent of dipolar polarization depends on the power of the electric field, the strength of dipole's moment, and the mobility of dipoles. Microwave energy loss through dielectric polarization results from the lag of polarization behind the change of the electric field. Dielectric polarization is the dominant process at high frequencies.

\section{Microwave Absorption}

At the macroscopic level, the microwave absorption by materials can be quantitatively stated as follows:

$$
P_{A}=2 \pi f \varepsilon_{0} \varepsilon_{\text {eff }}^{\prime}(E)^{2}
$$

Where $\mathrm{P}_{\mathrm{A}}$ is the average absorbed power per unit volume, $\mathrm{f}$ is the frequency of microwave, $\varepsilon$ is the permitivity of free space, $\varepsilon^{\prime}$ eff is the relative dielectric loss factor, and $\mathrm{E}$ is the internal electric field. An internal electric field $(\mathrm{E})$ is generated within the material when microwaves penetrate 
and propagate through a dielectric material. The internal electric field (E) is dependent on the relative dielectric constant $\varepsilon^{\prime}$ and $\varepsilon^{\prime}$ eff.

\section{Microwave Processing}

Microwave processing of materials is a thermally activated process based on microwave energy absorption. In contrast to all other conventional heating methods, microwave processing allows volumetric heating of materials. Microwave energy transforms into heat inside materials in microwave processing, which eliminates the need for spending energy on heating the walls of the furnace. Due to volumetric heating, the role of heat transfer becomes less important; materials can be quickly heated in microwave processing. These unique features of microwave processing result in significant reduction of energy consumption and processing duration, which plays a decisive role in most applications of microwave processing.

Microwave processing also has other benefits over conventional heating methods: rapid heating of thermal insulators such as ceramics and polymers, precise and controlled heating, material selective heating, reduction of hazardous emissions, increased product yield, environmentally friendly (clean and quiet), and cost savings.

In general, microwave processing will achieve energy savings, ranging from 10 to $90 \%$ higher efficiency compared with that of conventional heating. It is reported that the efficiency of microwave use increases significantly when the size of the load is increased. Besides the wellknown and extensive application in food manufacture and heating, microwave processing finds its way in many areas, which are roughly classified and summarized in Table 1.

\begin{tabular}{|l|l|}
\hline \multicolumn{2}{|c|}{ Table 1. Applications of microwave processing } \\
\hline Processes & \multicolumn{1}{c|}{ Examples } \\
\hline Recovery & $\begin{array}{l}\text { Waste remediation, extraction of Zinc from EAF dust, } \\
\text { Beneficiation of minerals }\end{array}$ \\
\hline Synthesis & $\begin{array}{l}\text { SiC synthesis, TiC synthesis, decomposition of PCS, } \\
\text { Catalysis and synthesis of organic compounds }\end{array}$ \\
\hline Removal of phases & $\begin{array}{l}\text { Drying and anhydration of material, solvent volatilization, } \\
\text { binder burnout }\end{array}$ \\
\hline Fabrication & Ceramic joining, CVI, coating, reduction of metal oxides \\
\hline Consolidation & $\begin{array}{l}\text { Si } 3 \mathrm{~N}_{4} \text { sintering, alumina sintering, melting, } \\
\text { Polymer curing, organic matrix composite curing }\end{array}$ \\
\hline Post-Fabrication treatments & $\begin{array}{l}\text { Annealing of Si } \mathrm{N}_{4} \text {, surface modification, nucleation / } \\
\text { crystallization, sealing }\end{array}$ \\
\hline Sample digestion & Mineral leaching \\
\hline
\end{tabular}




\section{Microwave Non-Thermal Effect}

Numerous observations have been reported in the literature of enhanced mass transport and enhanced reaction rates during microwave processing of various materials. These empirical observations of microwave enhancements have been broadly called microwave effect or microwave non-thermal effect. One of the phenomena about enhanced mass transport first observed by Janney, was the enhancement of oxygen diffusion in sapphire crystals heated in a 28 $\mathrm{GHz}$ microwave furnace. A $40 \%$ decrease in the apparent activation energy for bulk diffusion was observed under microwave processing as compared to conventional heating. One of the phenomena about enhanced reaction rates was observed by Gedye et al. in their investigation of organic reactions. They observed that microwave processing increases the reaction rate about 10 to 1200 times, as compared to conventional heating.

The existence of microwave non-thermal effect means many unique benefits, which microwave processing can provide but conventional heating can not. These benefits include not only the benefits mentioned earlier but also improved quality and properties of the processed materials. In Bykov's study on nanostructured porous alumina membranes, it was found that the porosity decreases consistently with increasing time of microwave processing, and that conventional heating causes no changes in the surface porosity of membranes.

Among the theoretical models for microwave non-thermal effect, the model based on the concept of non-equilibrium excitation seems natural and sound. The process of thermal energy absorption by materials is usually viewed as the excitation of lattice oscillations that have an equilibrium spectrum. Changes in the temperature of materials through thermally heating mean the slow evolution of the spectrum, which at all times remains in quasi-equilibrium. Microwave radiation not only increases the temperature of materials through microwave energy absorption, but also excites electron oscillations inside materials through interaction between electron and electromagnetic fields. Electron oscillations induce periodic distortion of the lattice potential and thereby modify the spectrum of lattice vibrations, that is, drive it from equilibrium. The fraction of energy stored in non-equilibrium excitations is determined by the time constant of the lattice vibrational modes and the electromagnetic field of microwave. The energy stored in nonequilibrium excitation is within the same order of magnitude as the energy of the electromagnetic field of microwave. Mass transport rate and reaction rate can be expressed in a general form as equation (3). Both the transport coefficient and the reaction coefficient are dependent on activation energy and temperature. The energy stored in non-equilibrium excitation will increase the activation energy for transport and reaction. The driving force is considered to be controlled by the concentration gradient or capillary stress, which are unaffected by microwave.

$$
\text { Rate }=(\text { driving force }) \times(\text { coefficient })
$$

\section{$\underline{\text { Microwave Processing Systems }}$}

A microwave processing system is made up of four basic components: power supply, magnetron, applicator (i.e., oven) for processing the target materials, and waveguide for transporting microwave from the magnetron to the applicator. 
Currently, the maximum output power of single magnetron produced is $90 \mathrm{~kW}$. Large microwave processing units can be built through connecting many magnetrons in parallel to multiply the power output.

Many companies such as Dennis Tool Co. (USA) and Fuji Denpa (Japan) offer standard microwave processing furnaces, and many companies will design and build a microwave processing system to meet their client's specification. Prices depend on the power output and frequency needed. The most inexpensive and common frequency used for microwave processing is $2.45 \mathrm{GHz}$.

Many systems used for microwave processing have been commercialized. An example of a commercial system is the Stoke-Type microwave system, which is developed by Dennis Tool Co. (USA) and is used to manufacture carbide.

\section{Task Discussions}

The following sections of this report describe the work conducted and the results obtained in each individual task. Each of the seven tasks addresses a separate aspect of the microwave steelmaking technology. 


\section{Task 1: Direct Steelmaking Tests and Technology Assessment}

The steelmaking tests and technology assessment consist of three aspects: 1 ) baseline microwave steelmaking; 2) bench-scale MW/EAF steelmaking; and 3) iron ore reduction by microwave assisted hybrid heating.

The baseline microwave steelmaking was designed to generate a series of steelmaking data from small samples using an available microwave sintering furnace. The baseline tests provide guidance for the bench-scale MW/EAF steelmaking tests. During execution of the project, we realized a need for microwave assisted hybrid heating in three scenarios: enhance performance of current heating equipment by adding microwave heating capability, utilize off-gases to improve energy efficiency, and reduce the need for high powered microwave generators. The study of iron ore reduction by hybrid heating was designed to generate some preliminary results for those considerations.

\subsection{Baseline Microwave Steelmaking}

Michigan Tech has a single mode microwave furnace manufactured by Wavemet. This furnace is capable of heating ceramic materials to over $1800^{\circ} \mathrm{C}$. We had conducted a preliminary steelmaking test using this equipment to prove the concept of microwave steelmaking before the project. This commercial furnace has a good microwave control system and generates repeatable results when processing small samples (a few grams). This equipment is an ideal tool for investigating various factors which may affect steelmaking using microwave. The steelmaking results generated from this equipment provided good reference for bench-scale MW/EAF steelmaking which is capable of processing 1000 grams of materials.

The factors which may affect steelmaking cover a broad range - from raw materials, process methods, and process parameters. In this study, we varied iron ore, coal, coal to iron ore ratio, lime to iron ore ratio, binder used to make pellets, compact density of pellets, and heating time for the microwave steelmaking tests. The produced steels and slags were examined to evaluate the quality of microwave produced steels. The data showed the relationships among those factors and provided guidance on how to reach an optimum steelmaking result using microwave heating.

\section{Experimental Method}

\section{$\underline{\text { Sample Designation }}$}

The sample's numbers were assigned as follows: the first letter denotes an iron ore type: Mmagnetite, H-hematite followed by a percentage number; the second letter denotes a reducing agent type: G-graphite, A-active carbon, C-coal followed by a percentage number; the third letter denotes a fluxing agent followed by a percentage number; the fourth letter denotes a binder followed by a percentage number; and the fifth letter denotes either compact pressure or microwave heating time. Figure 1.1 gives an example how a sample in this study was identified. 


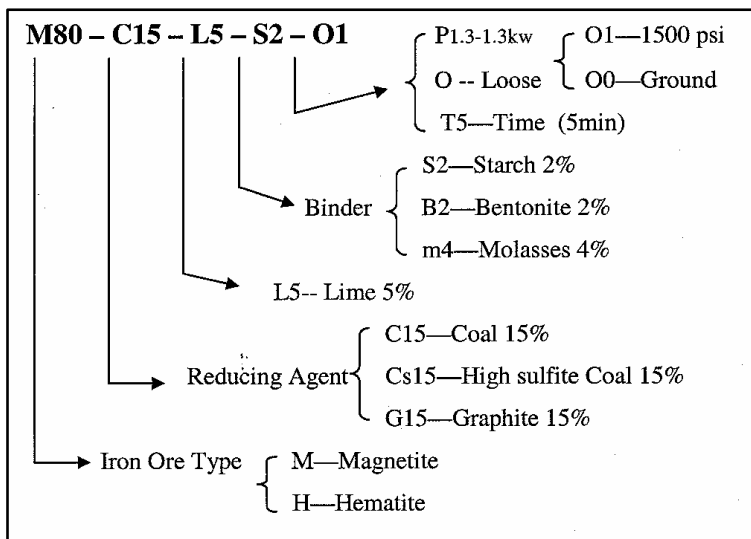

Figure 1.1. Sample Designation Explanation

\section{$\underline{\text { Raw Materials }}$}

Both magnetite and hematite ores, provided by Cleveland-Cliffs Inc. (CCI), were used in this study. The compositions are given in Table 1.1 . Both western coal and eastern coal were used as reducing agents in this study. Their compositions, as analyzed by Commercial Testing \& Engineering Co., are given in Table 1.2. The other raw materials used for this study include starch, bentonite, graphite powder, and lime. Their compositions are given in Table 1.3.

\section{$\underline{\text { Raw Materials Processing }}$}

The raw materials were processed to make feed materials for the microwave steelmaking tests. The procedure of the raw materials processing is given below.

1. Mix iron ore concentrate with lime and coal powders in different ratios. The binder is either starch or bentonite.

2. Compact the mixtures in a die under pressure of 1.5-3.0 ksi. Each compact weighs about 30 grams. The compacts were then dried in an oven.

3. Break the compact to pieces and passed them through a 10 mesh screen.

4. Charge 5-6 grams of such materials into small fireclay crucibles ready for microwave steelmaking tests.

\begin{tabular}{|l|c|c|c|c|c|c|c|c|}
\hline \multicolumn{10}{|c|}{ Table 1.1. Composition of the ores (\%) } \\
\hline Ore & $\mathbf{F e}$ & $\mathbf{S i O}_{\mathbf{2}}$ & $\mathbf{C a O}$ & $\mathbf{M g O}$ & $\mathbf{M n O}$ & $\mathbf{A l}_{\mathbf{2}} \mathbf{O}_{3}$ & $\mathbf{P}$ & $\mathbf{K}_{\mathbf{2}} \mathbf{O}$ \\
\hline Magnetite & 62.9 & 4.92 & 4.81 & 1.60 & 0.12 & 0.12 & 0.015 & 0.016 \\
\hline Hematite & 61.6 & 4.51 & 4.31 & 1.62 & & & 0.034 & \\
\hline
\end{tabular}

Direct Microwave Steelmaking

The equipment employed for microwave steelmaking was Wavemet MCR200 microwave furnace as shown in Figure 1.2. The steelmaking tests followed the procedure described below.

1. A sample in a small crucible was placed on the rotating stage of furnace chamber. The sample in rotating was heated by $1.4 \mathrm{~kW}$ microwave irradiation for 7 minutes in total, otherwise specified. The crucible outer surface reached a temperature about 1300$1400^{\circ} \mathrm{C}$.

2. After shutting off the microwave power, the crucible was moved out the furnace and cooled down in air. Normally a steel ball can be seen in the center of the crucible and it was surrounded by slag.

3. Steel ball and slag of the sample were separated and weighed respectively. By knowing the raw material compositions, the steel yield can be calculated. 


\begin{tabular}{|c|c|c|c|c|c|c|}
\hline \multicolumn{7}{|c|}{ Table 1.2. Composition of the Coal (\%) } \\
\hline & \multicolumn{2}{|c|}{ Western Coal } & \multicolumn{2}{|c|}{ Eastern Coal } & \multicolumn{2}{|c|}{ Warehouse Coal } \\
\hline & $\begin{array}{c}\text { As } \\
\text { received }\end{array}$ & Dry basis & $\begin{array}{c}\text { As } \\
\text { received }\end{array}$ & Dry basis & $\begin{array}{c}\text { As } \\
\text { received }\end{array}$ & Dry basis \\
\hline \multicolumn{7}{|c|}{ PROXIMATE ANALYSIS } \\
\hline Moisture & 1.61 & $\mathrm{XXXX}$ & 10.26 & $\mathrm{XXXX}$ & 12.66 & $\mathrm{XXXX}$ \\
\hline Ash & 9.73 & 9.89 & 11.51 & 12.83 & 8.05 & 9.22 \\
\hline Volatile & 29.76 & 30.25 & 32.09 & 35.76 & 38.72 & 44.33 \\
\hline Fixed Carbon & 58.90 & 59.86 & 46.14 & 51.41 & 40.57 & 46.45 \\
\hline $\mathrm{Btu} / \mathrm{lb}$ & 13230 & 13446 & 10050 & 11199 & 9041 & 10352 \\
\hline Sulfur & 1.01 & 1.03 & 3.40 & 3.79 & 0.79 & 0.91 \\
\hline MAF Btu & & 14922 & & 12847 & & 11403 \\
\hline \multicolumn{7}{|c|}{ ULTIMATE ANALYSIS } \\
\hline Moisture & 1.40 & $\mathrm{XXXX}$ & 10.26 & $\mathrm{XXXX}$ & 12.66 & $\mathrm{XXXX}$ \\
\hline Carbon & 72.72 & 73.75 & 57.58 & 64.16 & 55.15 & 63.14 \\
\hline Hydrogen & 4.76 & 4.81 & 3.83 & 4.27 & 3.63 & 4.16 \\
\hline Nitrogen & 1.38 & 1.40 & 1.04 & 1.16 & 0.79 & 0.90 \\
\hline Sulfur & 1.31 & 1.33 & 3.40 & 3.79 & 0.79 & 0.91 \\
\hline Ash & 10.68 & 10.83 & 11.51 & 12.83 & 8.05 & 9.22 \\
\hline Oxygen & 7.77 & 7.78 & 12.38 & 13.79 & 18.93 & 21.67 \\
\hline
\end{tabular}

\begin{tabular}{|l|c|c|c|c|c|c|c|c|c|}
\hline \multicolumn{10}{|c|}{ Table 1.3 Composition of Starch, Bentonite, Graphite and Lime (\%) } \\
\hline Element & Si & $\mathbf{M n}$ & $\mathbf{F e}$ & $\mathbf{M g}$ & $\mathbf{A l}$ & $\mathbf{T i}$ & $\mathbf{C a}$ & $\mathbf{N a}$ & $\mathbf{K}$ \\
\hline Starch & 3.01 & 0.01 & 0.31 & 0.02 & 0.18 & 0.00 & 0.09 & 0.40 & 0.67 \\
\hline Bentonite & 36.83 & 0.02 & 2.08 & 2.28 & 6.44 & 0.15 & 1.39 & 0.46 & 2.73 \\
\hline Graphite & 1.40 & 0.02 & 0.44 & 0.02 & 0.2 & 0.02 & 0.09 & 0.31 & 1.10 \\
\hline Lime & 1.51 & 0.02 & 0.29 & 0.42 & 1.28 & 0.02 & 40.76 & 0.56 & 2.55 \\
\hline
\end{tabular}

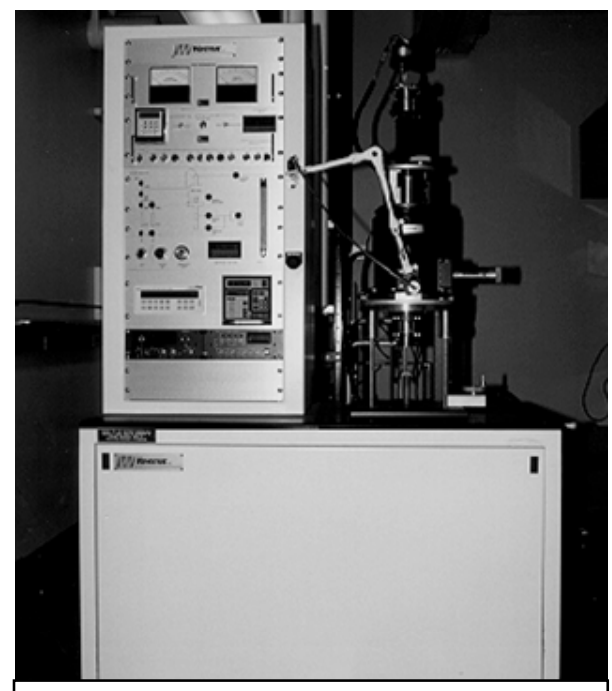

Figure 1.2. Wavemat MCR200 Microwave Furnace

\section{$\underline{\text { Sample Examination }}$}

The chemical compositions of the produced steels and slags were analyzed using an electron microscope (Super-Probe), inductively coupled plasma spectrometer (ICP), or a carbon and sulfur analyzer. The samples for Super Probe analysis were prepared according to the following procedure.

1. The samples were molded in plastic holders by epoxy resin. Each sample contained one piece of metal and four pieces of slag. Hardening time was about 24 hours.

2. The molded samples were cut and polished.

3. The instrument used for chemical analysis of the produced steel and slag was JXA-8600, 
SUPERPROBE. Three points of steel and three points of slag in each sample were probed, analyzed and averaged. The expected elements in steel were $\mathrm{Fe}, \mathrm{Al}, \mathrm{Mn}, \mathrm{P}, \mathrm{S}$, and $\mathrm{Si}$. The expected compounds in slag were $\mathrm{FeO}, \mathrm{SiO}_{2}, \mathrm{CaO}, \mathrm{P}_{2} \mathrm{O}_{5}, \mathrm{SO}_{3}, \mathrm{Al}_{2} \mathrm{O}_{3}, \mathrm{TiO}_{2}$, $\mathrm{Na}_{2} \mathrm{O}, \mathrm{K}_{2} \mathrm{O}, \mathrm{MnO}$, and $\mathrm{MgO}$.

A single steel or slag sample was not large enough for ICP or C\&S analysis. Three identical steelmaking tests were run to get three samples. The three samples were then treated together to meet the minimum analysis weight requirement.

\section{Results and Discussions}

More than 250 steelmaking tests have been run. Figure 1.3 is a view of the steelmaking samples. Figure 1.4 shows the cut section of the steel ball. Tables 1.4-1.6 present some steelmaking parameters and test results. The complete test parameters and results are given in Appendix A. Discussions on the test results follow.
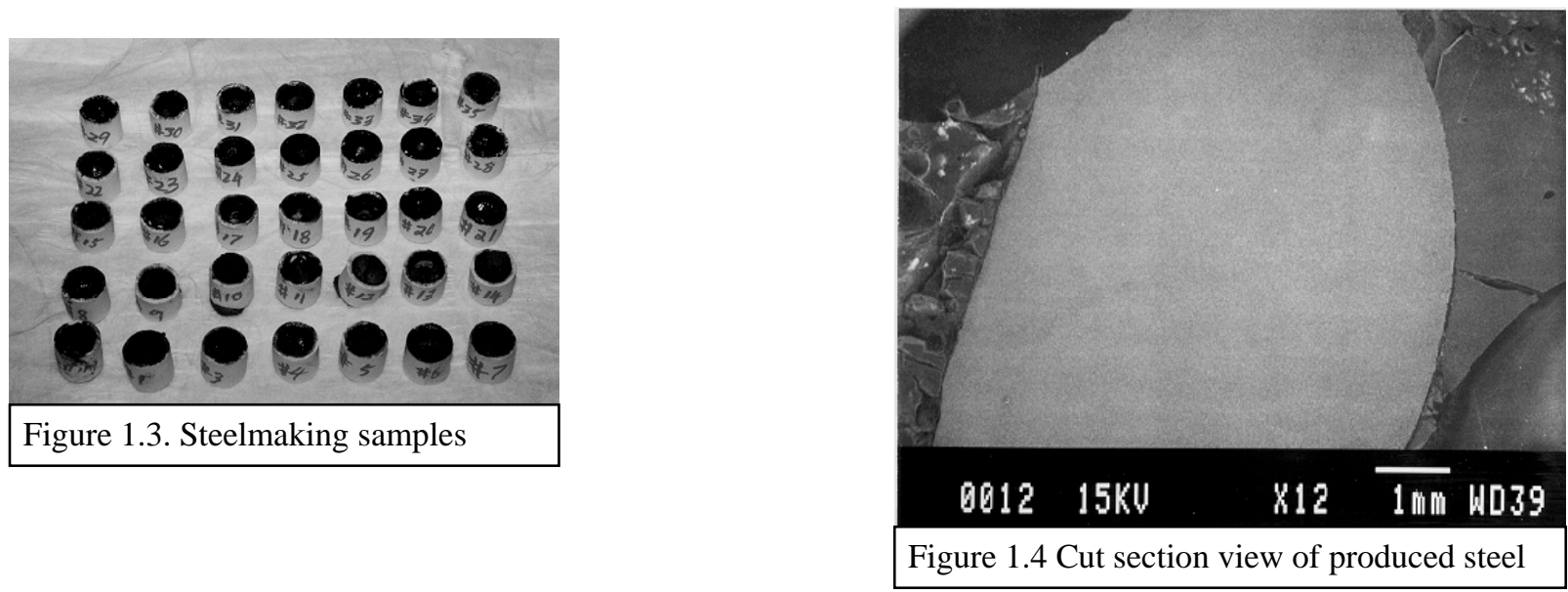

\begin{tabular}{|c|c|c|c|c|c|c|c|c|}
\hline \multicolumn{10}{|c|}{ Table 1.4 Pellet compositions and steel yields } \\
\hline Sample & $\begin{array}{c}\text { Iron Ore } \\
\mathbf{\%}\end{array}$ & $\begin{array}{c}\text { Reducing Agent } \\
\mathbf{\%}\end{array}$ & $\begin{array}{c}\text { Lime } \\
\mathbf{\%}\end{array}$ & $\begin{array}{c}\text { Binder, \% of total ore, } \\
\text { reductant and lime }\end{array}$ & $\begin{array}{c}\text { Slag } \\
\mathbf{W t} \mathbf{\%}\end{array}$ & $\begin{array}{c}\text { Steel Yield } \\
\mathbf{\%}\end{array}$ \\
\hline MG1 & 80 & carbon \#1 & 15 & 5 & starch & 3 & 23.3 & 95.59 \\
\hline MG5 & 81.6 & carbon \#1 & 13.8 & 4.6 & starch & 3 & 22.8 & 87.27 \\
\hline HG12 & 80 & carbon \#1 & 15 & 5 & starch & 3 & 23.6 & 92.22 \\
\hline MA4 & 78.4 & carbon \#2 & 16.2 & 5.4 & starch & 3 & 20.8 & 84.37 \\
\hline MA6 & 81.6 & carbon \#2 & 13.8 & 4.6 & starch & 3 & 41.6 & 72.77 \\
\hline MA8 & 80 & carbon \#2 & 15 & 5 & bentonite & 3 & 33.5 & 65.55 \\
\hline HA11 & 80 & carbon \#2 & 15 & 5 & starch & 3 & 28.7 & 89.40 \\
\hline MC10 & 80 & coal \#1 & 15 & 5 & bentonite & 3 & 43.2 & 74.93 \\
\hline HC13 & 80 & coal \#1 & 15 & 5 & starch & 3 & 45.4 & 72.09 \\
\hline MHC14 & 80 & coal \#1 & 15 & 5 & \multicolumn{1}{|c|}{ starch } & 3 & 46.8 & 70.10 \\
\hline $\begin{array}{l}\text { Magnetite ore, microwave heating only } \\
\text { carbon \#1 is graphite (fixed C>90\%), carbon \#2 is activated carbon (fixed C>90\%) }\end{array}$ \\
\hline
\end{tabular}




\begin{tabular}{|c|c|c|c|c|c|c|}
\hline \multicolumn{7}{|c|}{ Table 1.5 Steel composition } \\
\hline Sample & Fe & P & Al & Si & Mn & S \\
\hline MG1 & 99.175 & 0.023 & 0 & 0.807 & 0 & 0.002 \\
\hline MG5 & 99.301 & 0.026 & 0 & 0.643 & 0.021 & 0.009 \\
\hline HG12 & 99.167 & 0.073 & 0 & 0.713 & 0.045 & 0.002 \\
\hline MA4 & 94.030 & 0.205 & 0 & 5.742 & 0.025 & 0.007 \\
\hline MA6 & 99.861 & 0.045 & 0 & 0.010 & 0.031 & 0.053 \\
\hline MA8 & 99.220 & 0.084 & 0 & 0.668 & 0.024 & 0.004 \\
\hline HA11 & 99.804 & 0.176 & 0 & 0.023 & 0 & 0.043 \\
\hline MC10 & 99.963 & 0.039 & 0 & 0.002 & 0 & 0.013 \\
\hline HC13 & 99.956 & 0.033 & 0 & 0 & 0 & 0.017 \\
\hline MHC14 & 99.936 & 0.023 & 0 & 0.018 & 0.005 & 0.018 \\
\hline \multicolumn{7}{|c|}{ (Magnetite ore, microwave heating only) } \\
\hline
\end{tabular}

\begin{tabular}{|l||c|c|c|c||c|c|c||c|c||c|c|}
\hline \multicolumn{10}{|c|}{ Table 1.6 Slag composition } \\
\hline Sample & $\mathbf{S i O}_{2}$ & $\mathbf{C a O}$ & $\mathbf{F e O}$ & $\mathbf{M g O}$ & $\mathbf{A l}_{\mathbf{2}} \mathbf{O}_{\mathbf{3}}$ & $\mathbf{P}_{\mathbf{2}} \mathbf{O}_{\mathbf{5}}$ & $\mathbf{S O}_{\mathbf{3}}$ & $\mathbf{T i O}_{\mathbf{2}}$ & $\mathbf{N a}_{\mathbf{2}} \mathbf{O}$ & $\mathbf{K}_{\mathbf{2}} \mathbf{O}$ & $\mathbf{M n O}$ \\
\hline MG1 & 58.027 & 27.519 & 0.579 & 4.243 & 8.786 & 0.014 & 0.007 & 0.041 & 0.415 & 0.278 & 0.09 \\
\hline \hline MG5 & 50.481 & 34.597 & 2.349 & 3.637 & 7.861 & 0.006 & 0.017 & 0.165 & 0.357 & 0.233 & 0.297 \\
\hline \hline HG12 & 50.193 & 33.86 & 1.546 & 4.637 & 8.348 & 0.002 & 0.009 & 0.248 & 0.32 & 0.223 & 0.612 \\
\hline MA4 & 58.294 & 25.619 & 0.384 & 3.352 & 10.78 & 0 & 0.414 & 0.182 & 0.466 & 0.303 & 0.209 \\
\hline MA6 & 42.782 & 24.331 & 22.22 & 1.988 & 7.525 & 0.064 & 0.046 & 0.112 & 0.478 & 0.236 & 0.22 \\
\hline \hline MA8 & 49.528 & 34.539 & 2.413 & 3.646 & 8.452 & 0.017 & 0.142 & 0.133 & 0.583 & 0.252 & 0.295 \\
\hline HA11 & 54.307 & 21.494 & 8.613 & 4.061 & 9.674 & 0.066 & 0.048 & 0.272 & 0.547 & 0.268 & 0.65 \\
\hline MC10 & 58.954 & 14.266 & 13.55 & 2.145 & 9.844 & 0.062 & 0.036 & 0.081 & 0.635 & 0.322 & 0.1 \\
\hline \hline HC13 & 44.491 & 19.609 & 23.67 & 2.355 & 8.5 & 0.092 & 0.085 & 0.155 & 0.411 & 0.239 & 0.393 \\
\hline MHC14 & 47.690 & 16.817 & 24.33 & 2.067 & 7.859 & 0.046 & 0.08 & 0.151 & 0.514 & 0.22 & 0.225 \\
\hline (Magnetite ore, microwave heating only) & & & & & \\
\hline
\end{tabular}

\section{$\underline{\text { Steel Yield }}$}

The steel yield varied from 0 to $100 \%$. Steel yield mainly depends on the amount of reducing agent added into the iron ore pellets. More accurately speaking, steel yield depends on the iron oxide content of iron ore and the amount of fixed carbon in a carbonaceous reducing agent. No matter what type of reducing agent was used, selected from western coal, eastern coal, graphite powder or active carbon powder, any of the reducing agents can generate steel yield close to $100 \%$, if enough fixed carbon exists. A low ratio of reducing agent results in low steel yield. However, the type of binder may have some effect on yield. With the other components held constant, using starch as the binder results in higher steel yield than bentonite and molasses. This is due to the fixed carbon contained in starch. Another phenomenon worth noticing is that there exists a critical ratio of carbon in the iron ore pellets. If the pellets contain too high of a ratio of 
carbon, normal steel balls and slag do not form during steelmaking. No matter what reducing agent is used, the critical percentage of the fixed carbon contained in the pellets is about $15 \%$ for the tested magnetite and hematite iron ores. That explains why more eastern coal, which contains less fixed carbon, is needed to get the same level of steel yield.

\section{$\underline{\text { Sulfur in the Steel }}$}

Sulfur content in steel generally originates from coal. The amount of sulfur in the steel varies with the type of coal and the ratio of coal in the pellets as well. High sulfur containing coal and high amount of coal addition causes higher $\mathrm{S}$ content in the steel. For the western coal, the average S content fluctuates between $0.3 \% \sim 0.5 \%$. For the high S content Eastern coal, however, the concentration of $\mathrm{S}$ reaches as high as $1.14 \% \sim 1.57 \%$, while the graphite reduced steel contains only $0.027 \% \sim 0.037 \%$ sulfur. Figure 1.5 illustrates the relationship of the steel sulfur content with reducing agent types and their additions.

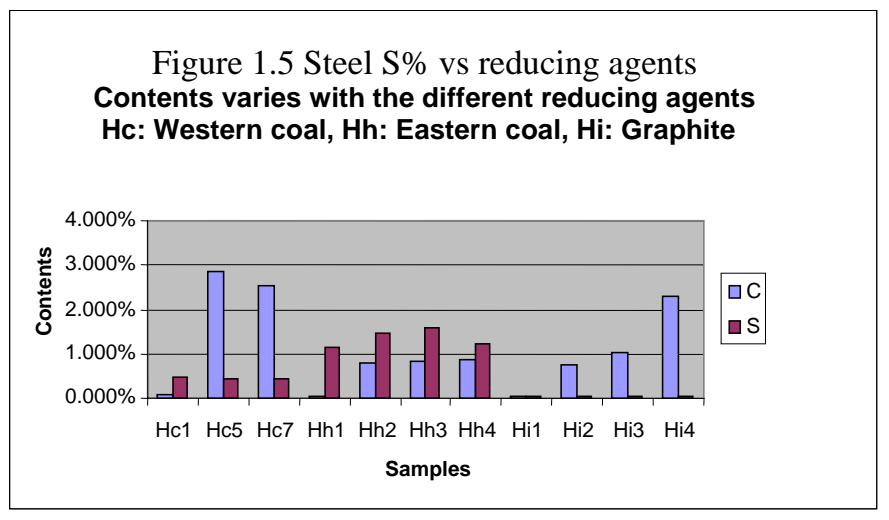

Promisingly, the high ratios of lime in the sample can significantly reducing the $\mathrm{S}$ content. By increasing lime ratio from $3 \%$ to $15 \%$, the S content reduced from $0.50 \%$ to $0.16 \%$ as illustrated by Figure 1.6. Figure 1.7 shows the effect of lime addition on the sulfur content of slag. It is obvious that sulfur moves from steel into slag if lime addition is increased.
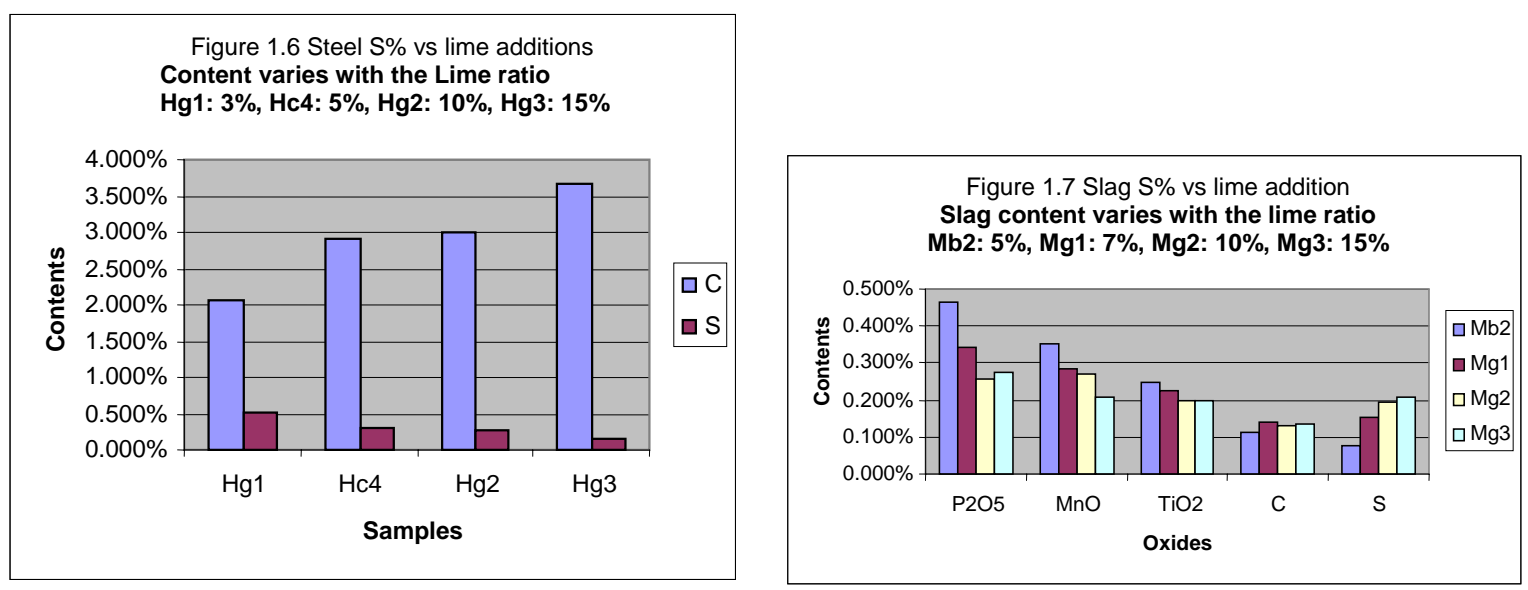

\section{Carbon in the Steel}

Carbon concentration in the steel is effected in several ways. First, the ratio of carbon in the pellets can affect it. Carbon concentration increases if the ratio of carbon in the pellets increases. However, this increase stops after the ratio of carbon reaches a certain level, as illustrated in Figure 1.8. Second, extended heating time has little effect on $\mathrm{C}$ content as seen in Figure 1.9. Third, $\mathrm{C}$ content varies closely with the ratio of lime. High lime ratio correlates to a high $\mathrm{C}$ 
content. The C content goes up from $2.05 \%$ to $3.66 \%$, while lime ratio increases from $3 \%$ to $15 \%$ as shown in Figure 1.6.
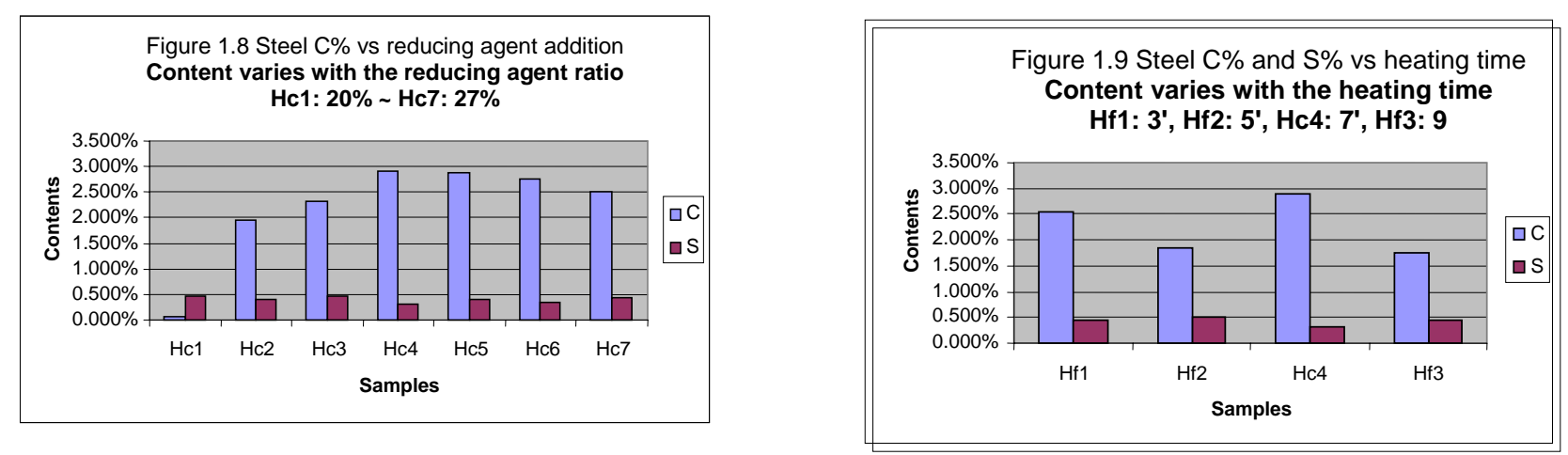

\section{$\underline{\text { Phosphorus in the Steel }}$}

Phosphorus content in the steel mainly comes from iron ore. But its concentration is higher than that of iron ore because the weight of the produced steel is less than that of the iron ore. Phosphorus content changes with steel yield. Higher steel yield results in higher P content. An interesting phenomenon is that the $\mathrm{P}$ content in the steel varies with the microwave heating power. Low microwave power correlates to low concentration of P. The P concentration in the steel is around 0.02 to 0.05 .

\section{Effects of Iron Ore Types}

There is no significant difference between steels made of magnetite and hematite. There is not much difference in slag composition either. The only noticeable difference is that the $\mathrm{TiO}_{2}$ and $\mathrm{MnO}$ content in hematite slag are approximately three times higher than those in magnetite slag.

\section{$\underline{\text { Effects of Binder Types }}$}

Starch can provide some extra fixed carbon for iron ore reduction. In the situation where insufficient fixed carbon is provided by coal, the starch in the pellets shows assistance to iron ore reduction. The steel yield will be higher and FeO in slag will be lower.

\section{$\underline{\text { Pellet Compact Density }}$}

Among the three groups of samples (loose powder feed, $1.5 \mathrm{ksi}$ and $3.0 \mathrm{ksi}$ compacted agglomerates), there was no noticeable difference in steel yield, carbon and sulfur content.

\section{Conclusion}

The type of reducing agent has a significant effect on the steel yield. Steel yield strongly depends on the fixed carbon of a carbonaceous reducing agent. The fixed carbon must be more than a critical value in order to obtain high steel yield. Graphite has the highest fixed carbon. The least amount of graphite is required to achieve a high steel yield in comparison with coals. Enough 
addition of any carbonaceous reducing agent can result in a steel yield close to $100 \%$. However, an excess amount of fixed carbon is detrimental to steel and slag separation and wastes energy. Volatiles in coal escape at temperatures below iron ore reduction temperature. Therefore, the volatiles do not participate in iron ore reduction. It is a big energy waste.

The carbon content of produced steel varies from to $0.026 \%$ to over $3 \%$ depending on the amount of fixed carbon in steelmaking raw materials.

The sulfur content of produced steel varies from $0.002 \%$ to over $1 \%$. The sulfur primarily comes from coal. Low sulfur carbonaceous reducing agent results in low sulfur steel. Sulfur in steel can be removed by adding lime into the steelmaking raw materials.

The phosphorus content in microwave produced steel varies from $0.023 \%$ to $0.193 \%$. Phosphorus in steel comes from steelmaking raw materials.

There are no surprising differences in steel and slag produced by microwave steelmaking compared to steel and slag produced by conventional steelmaking. The microwave produced steel is of good quality. 


\subsection{Bench-Scale MW/EAF Steelmaking}

\section{Microwave Assisted Electric Arc Furnace Design}

The bench-scale microwave assisted electric arc furnace (MW/EAF) is the key apparatus for investigating and optimizing the processes involved in the new steelmaking technology. The bench-scale MW/EAF system consists of microwave heating subsystem, electric arc heating subsystem, cooling subsystem, emission evacuation subsystem, refractory vessel (crucible), insulating materials and furnace shell. The structure of the MW/EAF furnace is illustrated schematically in Figure 1.10 .

The microwave heating subsystem includes six parallel sets of microwave generators, waveguide, electric current monitor and control devices. Every microwave generator consists of accessory components and one water-cooled magnetron, which is a commercial SHARP RVMZ A296WRL magnetron with fixed output power of $1000 \mathrm{~W}$ and frequency of $2.45 \mathrm{GHz}$. Microwave radiation is delivered into the furnace cavity through the waveguides from three lateral sides of the furnace for homogeneous microwave heating.

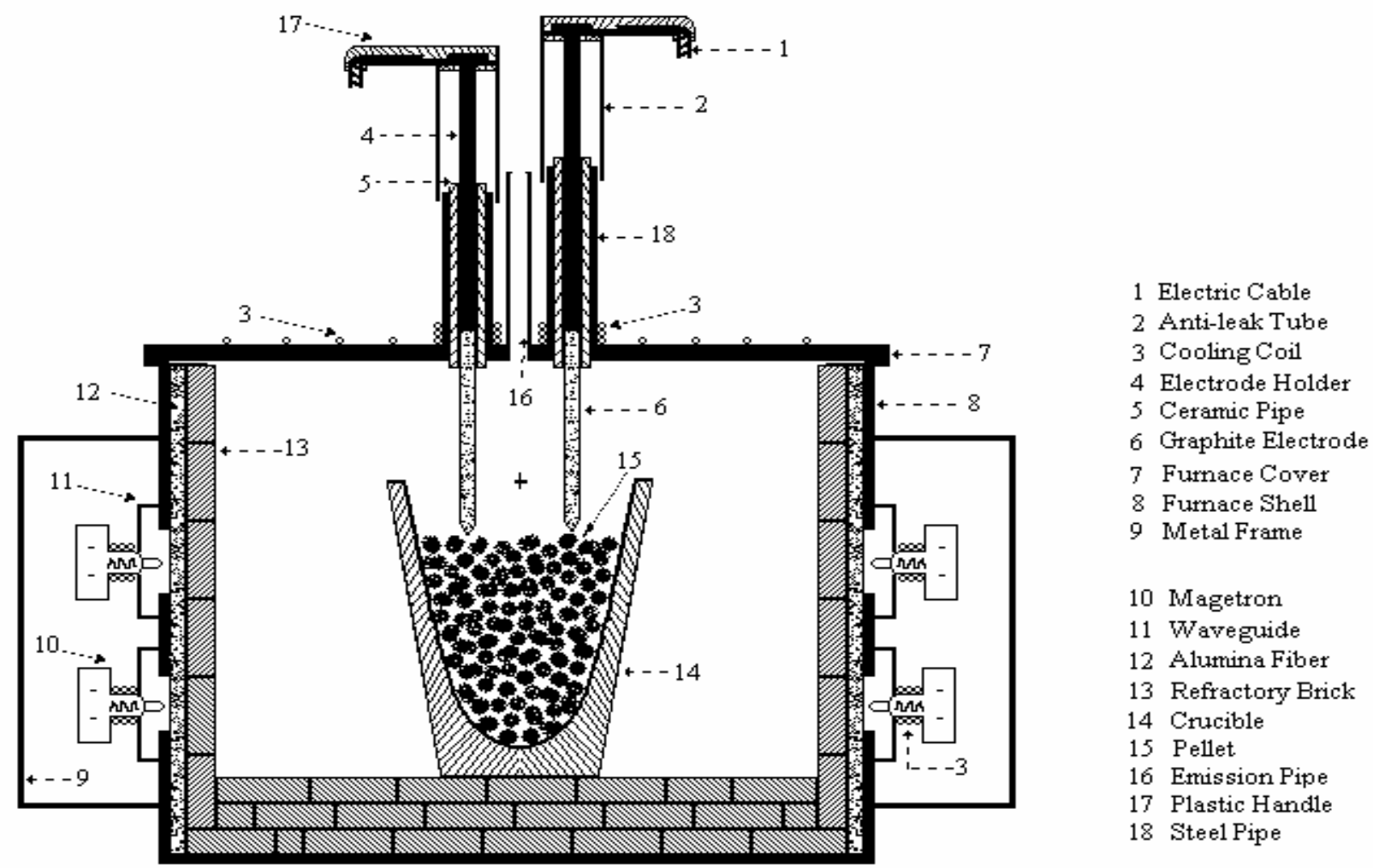

Figure 1.10 Schematic diagram of microwave assisted electric arc furnace

The electric arc heating subsystem mainly includes a low-voltage electric power system and two graphite electrodes. The electric power system is a Hobart Brothers Company's Arc Welder 
(Model ML-304), with adjustable supply of AC voltage and maximum supply voltage of $100 \mathrm{~V}$. The operating voltage and current are monitored by two indicating meters.

The cylindrical graphite electrode is 0.5 " in diameter and 24 ' ' in length. The electrodes are manually controlled by furnace operators, and can only move vertically during operation. The ceramic pipe around the cylindrical electrode serves as an electric and thermal insulator. The steel pipes around the electrodes are shrouded by plastic tapes for electric insulation and safety.

The cooling subsystem includes air-cooling and water-cooling subsystems. Lab compressed air is ducted into the three outside frames of the furnace and blown on the microwave generators for temperature control. The water-cooling subsystem provides more effective temperature control to some critical components of the furnace. Water-cooled copper coils were welded onto the magnetron tubes, the furnace cover, and the lower part of the steel pipes around the electrodes for better cooling.

The interior wall and bottom of the furnace consist of refractory bricks and alumina fibers. Those refractory materials can withstand the variety of destructive influences caused by elevated temperature; they also protect the furnace shell and prevent it from absorbing excessive heat.

The emission evacuation subsystem includes an exhaust pipe welded on the furnace cover, a steel duct, an exhaust fan and a laboratory ventilation system. During operation, the exhaust fan withdraws gases from the furnace cavity to the ventilation system through the steel duct connected to the pipe. Gas analytical equipment can be connected to the pipe through a three way pass.

During operation, one refractory crucible contains the charge (i.e. pellets) and is placed in the center of the furnace cavity. There is a viewing window in the furnace cover, through which the furnace operator can observe the charge and precisely control the position of the electrodes.

The features of the bench-scale MW/EAF furnace offer great convenience for the investigation of direct steelmaking. During operation, only graphite electrodes are consumed and no metal is dissolved. It is easy to obtain the actual value of the yield of iron ores. The allowance for the vertical movement of both electrodes is large enough so that the effect and operation of electric arcing can be readily controlled. The special design in joining graphite electrodes with the metal holders almost eliminates the problem of electrode breakage.

\section{Experimental Method}

\section{Apparatus}

Reducing magnetite ore through microwave heating and electric arcing methods was carried out in the bench-scale MW/EAF furnace. Temperature measurements were made with Omega Engineering Inc.'s infrared thermometer (Model OS3722). 


\section{$\underline{\text { Materials }}$}

Ten pellet samples with different compositions and different binders were used. The compositions of the pellet samples in weight ratio and in weight percentage are respectively listed in Table 1.7 and Table 1.8. Magnetite ore concentration was grade B magnetite from Prince Manufacturing Company. The powdered coal was high volatile bituminous coal. The compositions of raw materials are listed in Table 1.9 and Table 1.10. Magnesium oxide used as crucible lining material has 99\% purity.

\begin{tabular}{|c|c|c|c|c|c|c|}
\hline \multicolumn{7}{|c|}{ Table 1.7. Pellet composition by weight ratio } \\
\hline Pellet & Magnetite & Bentonite & Molasses & Starch & Lime & Coal \\
\hline$\# 1$ & 100 & 5 & 0 & 0 & 10 & 20 \\
\hline$\# 2$ & 100 & 5 & 0 & 0 & 10 & 40 \\
\hline$\# 3$ & 100 & 0 & 0 & 10 & 5 & 50 \\
\hline$\# 4$ & 100 & 0 & 0 & 7.5 & 10 & 25 \\
\hline$\# 5$ & 100 & 0 & 6 & 0 & 10 & 25 \\
\hline$\# 6$ & 100 & 0 & 0 & 7.5 & 25 & 45 \\
\hline$\# 7$ & 100 & 0 & 6 & 0 & 35 & 45 \\
\hline$\# 8$ & 100 & 0 & 0 & 7.5 & 35 & 45 \\
\hline$\# 9$ & 100 & 0 & 0 & 7.5 & 10 & 45 \\
\hline$\# 10$ & 100 & 0 & 0 & 7.5 & 15 & 45 \\
\hline
\end{tabular}

\begin{tabular}{|c|c|c|c|c|c|c|}
\hline \multicolumn{7}{|c|}{ Table 1.8. Pellet composition by weight percentage } \\
\hline Pellet & Magnetite & Bentonite & Molasses & Starch & Lime & Coal \\
\hline$\# 1$ & 74.07 & 3.70 & 0 & 0 & 7.41 & 14.81 \\
\hline$\# 2$ & 64.52 & 3.23 & 0 & 0 & 6.45 & 25.81 \\
\hline$\# 3$ & 60.61 & 0 & 0 & 6.06 & 3.03 & 30.30 \\
\hline$\# 4$ & 70.18 & 0 & 0 & 5.26 & 7.02 & 17.54 \\
\hline$\# 5$ & 70.92 & 0 & 4.26 & 0 & 7.09 & 17.73 \\
\hline$\# 6$ & 56.34 & 0 & 0 & 4.22 & 14.08 & 25.35 \\
\hline$\# 7$ & 53.76 & 0 & 3.23 & 0 & 18.81 & 24.19 \\
\hline$\# 8$ & 53.33 & 0 & 0 & 4.00 & 18.66 & 24.00 \\
\hline$\# 9$ & 61.53 & 0 & 0 & 4.62 & 6.15 & 27.69 \\
\hline$\# 10$ & 59.70 & 0 & 0 & 4.48 & 8.96 & 26.86 \\
\hline
\end{tabular}

\begin{tabular}{|l|c|c|c|c|c|c|c|c|c|}
\hline \multicolumn{10}{|c|}{ Table 1.9. Chemical composition of raw materials (wt \%) } \\
\hline & Si & $\mathbf{M n}$ & $\mathbf{F e}$ & $\mathbf{M g}$ & $\mathbf{A l}$ & $\mathbf{T i}$ & $\mathbf{C a}$ & $\mathbf{N a}$ & $\mathbf{K}$ \\
\hline Magnetite ore & 3.27 & 0.09 & 58.65 & 0.56 & 1.67 & 2.55 & 0.25 & 0.42 & 0.76 \\
\hline Starch & 3.01 & 0.01 & 0.31 & 0.02 & 0.18 & 0 & 0.09 & 0.40 & 0.67 \\
\hline Bentonite & 36.83 & 0.02 & 2.08 & 2.28 & 6.44 & 0.15 & 1.39 & 0.46 & 2.73 \\
\hline Lime & 1.51 & 0.02 & 0.29 & 0.42 & 1.28 & 0.02 & 40.76 & 0.56 & 2.55 \\
\hline
\end{tabular}




\begin{tabular}{|l|c|c|c|c|c|}
\hline \multicolumn{7}{|c|}{ Table 1.10. Composition of coal (wt \%) } \\
\hline & Moisture & Ash & Volatile materials & Fixed Carbon & Sulfur \\
\hline As Received & 1.61 & 9.73 & 29.76 & 58.90 & 1.01 \\
\hline Dry Basis & 0 & 9.89 & 30.25 & 59.86 & 1.03 \\
\hline
\end{tabular}

\section{Procedures}

\section{Reduction of Pellet Sample in MW/EAF}

$800 \mathrm{~g}$ or $600 \mathrm{~g}$ pellet samples were weighed and put into a crucible. The crucible was placed in the center of the MW/EAF furnace cavity. After the cooling subsystem and emission evacuation subsystem of the MW/EAF furnace were turned on, microwave heating was started. The pellet sample was heated for up to 20 minutes. Electric arcing then started. The applied direct voltage reached $50 \mathrm{~V}$. At the beginning, graphite electrodes were positioned to contact the top layer of the pellet sample, and the electric arc current was set to $150 \mathrm{~A}$. After the pellet sample melted down, the graphite electrodes were lowered slowly to keep the electric arc current constant. When slag and molten steel were formed and the required heating time was reached, arc heating and microwave heating were turned off. Through the watch window of the MW/EAF furnace, the temperature was measured immediately. Then the crucible was taken from the furnace to cool.

\section{Coating Crucible Internal Surface}

- Crush lime or magnesium oxide

- Mix lime or magnesium oxide with a binder and water

- Stir mixture until it is uniform

- Coat the interior of crucible with the slurry evenly

- Dry crucible in an oven at temperature $100{ }^{\circ} \mathrm{C}$ for 24 hours

\section{Results and Discussion}

The experimental parameters and results of magnetite reduction through microwave and electric arc heating are summarized in Appendix B. Figures 1.11 to 1.14 provide several views during a

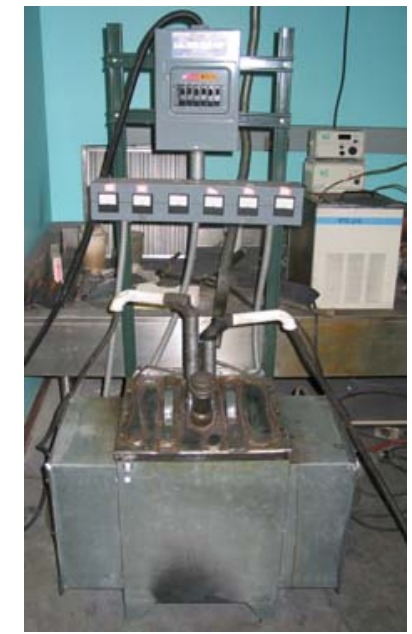

Novel Direct Steelmaking by Combining Microwave, Electric Arc, and Exothermal Heating Technologies DE-FC36-01ID14209, Final Technical Report steelmaking test. Figure 1.11 is the bench-scale MW/EAF furnace used for the steelmaking tests. Figure 1.12 shows a technician loading the fireclay crucible containing iron ore pellets into the furnace chamber. Figure 1.13 shows the molten slag and steel in the crucible upon completion of a steelmaking run. Figure 1.14 shows the produced steel.

Figure 1.11. Bench-scale MW/EAF furnace

Figure 1.12. Technician loading fireclay crucible into the furnace chamber

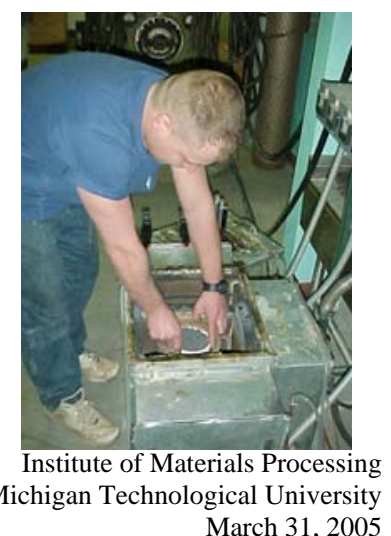




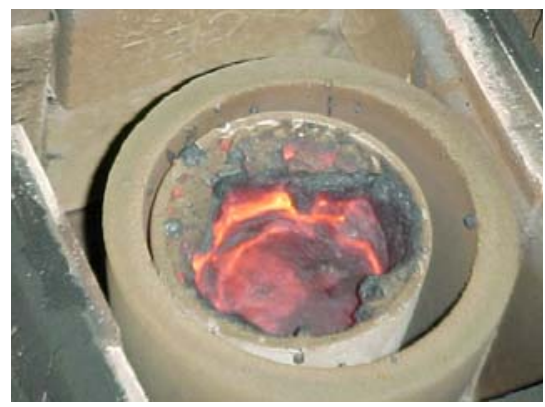

Figure 1.13. After

completion of electric arcing

Figure 1.14. MW/EAF produced steels

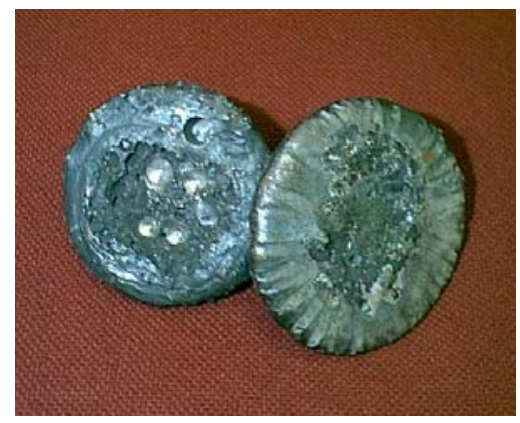

Binders and Lime in Pellet Sample

The weight percentages of the three binders in pellet samples were similar, but their bonding capabilities were very different. Dried pellet samples with starch binder had excellent strength and toughness. The pellets were still in good shape after storing for a long time. Excessive lime addition above $25 \%$ deteriorated the pellet properties significantly. None of the experiments showed any obvious effect from different binders on the steel yield.

\section{Temperature}

Temperature measurements indicates that after microwave heating for about 5-7 minutes, pellet samples reach temperatures between $850^{\circ} \mathrm{C}-900^{\circ} \mathrm{C}$ and remained in that temperature range until electric arc heating started. The temperature of the molten materials during the final stage of electric arc heating is in the range of $1350-1500^{\circ} \mathrm{C}$.

\section{Start Time of Electric Arcing}

The experimental results indicate that electric arc heating could be applied to heat a pellet sample (\#3) after it was processed for as short as 7 minutes by microwave heating. Microwave heating for more than 10 minutes guaranteed the start of electric arc heating on the pellet sample (\#3) every time. After microwave heating for 20 minutes, most pellet samples were heated successfully by electric arcing. Pellet samples with a lime ratio of 35/100 had difficulty of electric arcing.

The minimum start time of electric arcing to a specific pellet sample relates to the applied voltage and the electric conductivity of the pellet sample, which depends on the percentage of reduced iron and the composition of the pellet sample. High applied AC voltage makes the start of the electric arc easier, such as 200-800 V in an industrial EAF. Lime particles are not melted during microwave heating. These particles prevent good contact and growth of reduced iron particles. Excessive lime in pellet samples increased the minimum start time of electric arcing.

In order to make an early start of electric arcing, the lime content of the pellet sample should be less than $25 \%$.

\section{$\underline{\text { Steel Yield }}$}

Experimental results indicate that steel yield approaching $100 \%$ can be achieved for a pellet sample with appropriate composition after 6 minutes of electric arcing. Electric arc heating time 
and composition of the pellet sample significantly affect the steel yield of the new steelmaking process.

Figure 1.15 illustrates the effects of electric arcing time and pellet sample's composition on steel yields. It is indicated that steel yield can be increased by increasing electric arcing time. During the period of electric arcing, reduced iron particles melt and separate from slag. After melting is complete, a certain holding time is required for complete separation. The separation contributes to steel yield increase. Long electric arcing time guarantees an enough holding time for separation.

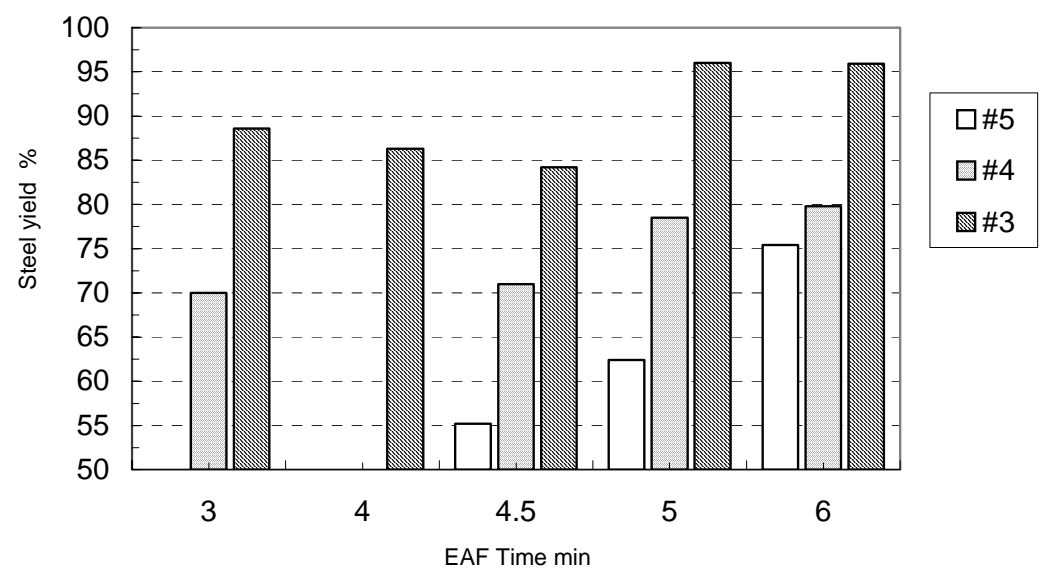

Many pellet samples with appropriate composition can

Figure 1.15. Effects of electric arcing time and composition on steel yield achieve steel yield above 95\% after 6 minutes of arcing. Therefore electric arcing time for the bench-scale MW/EAF furnace can be set at 6 minutes.

Electric arcing time could be reduced significantly by replacing the present AC electric arcing subsystem with ultra high DC voltage equipment. Two changes contribute to the decrease of electric arcing time under high voltage. The time to melt a pellet sample is decreased due to rapid heating and the time to complete reduction is decreased because most of the iron ore is reduced at high temperature.

Figure 1.15 also illustrates that steel yields of pellet samples with different compositions are different even when electric arcing time is the same. High steel yield can be achieved by using a pellet sample with more coal or starch, which serves as both a binder and as a reductant.

Figure 1.16 illustrates the variation of steel yield with respect to coal content of pellet samples. Pellet samples with high coal content generally guarantee high steel yield.

Coal serves as a reductant in the new steelmaking process. Variations of steel yield with coal content of pellet samples mainly result from incomplete reduction of iron ore due to lack of coal. If coal in the pellet sample is greatly more than necessary, both MW and EAF heating

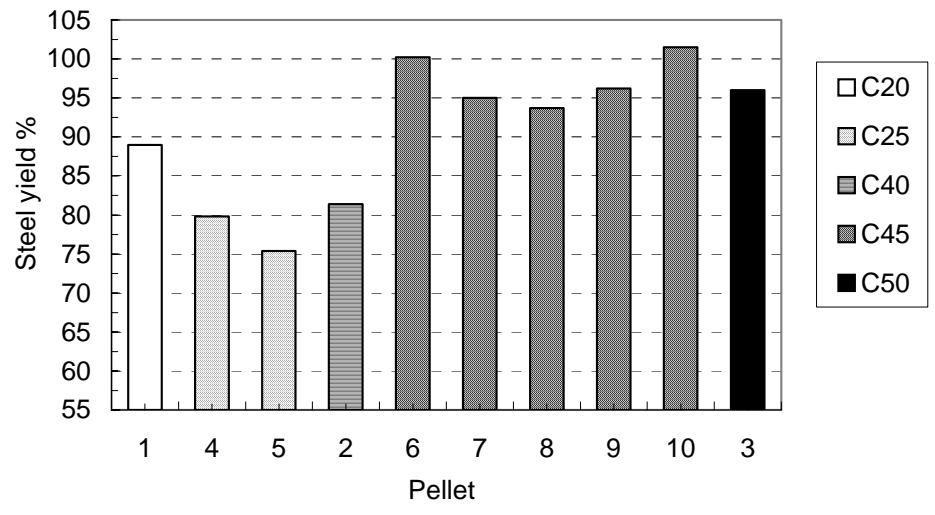

Figure 1.16 Variation of steel yield with coal 
times need to be increased. Microwave heating time needs to be increased because coal has a more inferior microwave absorbing capability than magnetite ore. Excessive coal mixed with slag increases the slag's viscosity, which retards steel separation from the slag. In order to achieve a reasonable steel yield, electric arcing time has to be increased.

Powdered coal primarily consists of volatile materials, fixed carbon, and ash. Volatiles come out of coal and escape from the furnace during early stages of MW heating. The volatiles do not contribute to heating the pellet samples or to their reduction. It was totally wasted. In the reduction process, some fixed carbon is transferred through the Boudouard reaction into carbon monoxide, which reduces magnetite ore into iron. Some fixed carbon is oxidized into carbon dioxide and then emitted as exhaust gas. A small amount of fixed carbon is ejected out of the crucible due to arcing.

The ratio of the fixed carbon as reductant to the fixed carbon wasted depends on the operating temperature, the ratio of microwave heating time and electric arcing time, and the operating conditions of the furnace. Elevated temperature can increase both the rates of the Boudouard reaction and the reduction reaction, so fixed carbon can be utilized more efficiently under high operating temperature. Electric arcing can easily and rapidly elevate the temperature of the furnace charge. Different conditions of furnace operation related to the emission evacuation subsystem can affect reaction rates by varying the concentrations of gases inside the furnace cavity.

The theoretical minimum ratio of coal to magnetite for complete reduction is from 9.52 to 14.03 depending on the fixed carbon content of coal and iron ore quality (see Task 5: Energy Assessment). The coal to magnetite ratio under present experimental conditions is 20/100 to $50 / 100$, so there is great room to reduce the amount of coal used in MW/EAF process by optimizing furnace operation and careful control of raw materials.

Figure 1.17 illustrates the variation of steel yield with respect to the lime content of the pellet sample. Figure 1.16 indicates that the effect of lime content on steel yield is not significant. When compared with the effect of coal content on steel yield, the effect of lime content is not significant. When lime content varies from $5 / 100$ to $35 / 100$, the steel yield only varies about $6 \%$.

Lime content affects steel yield by altering slag viscosity. Pellet samples with different lime contents produce slags with different composition,

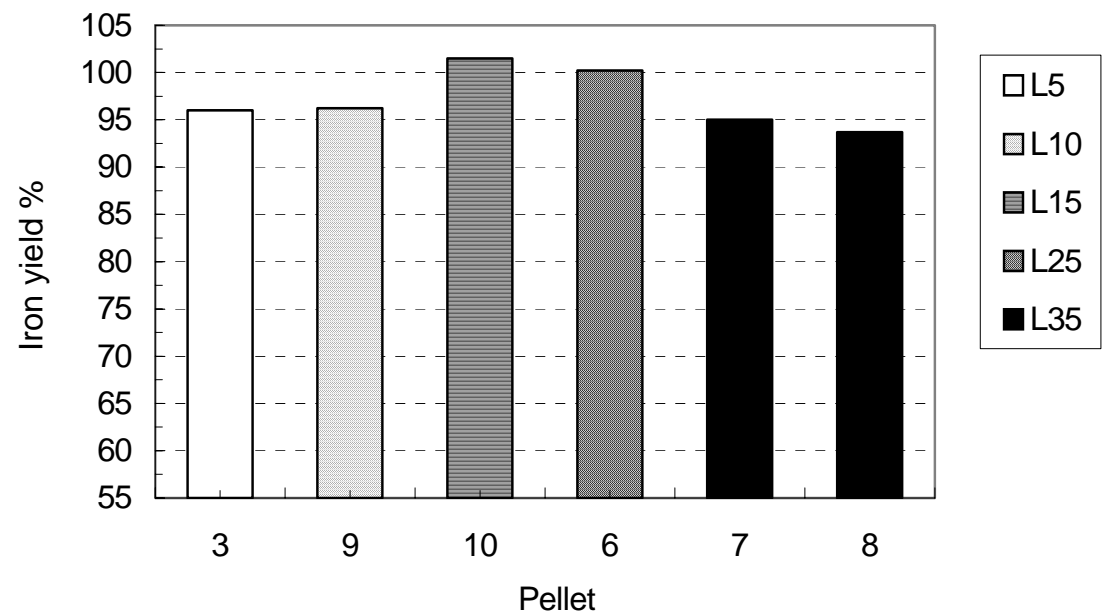

Figure 1.17. Variation of steel yield with lime 
which have different viscosity at the same temperature; therefore, the extent of steel separation from slag is correlated with the lime content of the pellet sample.

$\underline{\text { Summary }}$

Steel yield of more than 95\% can be easily achieved using the MW/EAF process, therefore this direct steelmaking technology is practical for producing steel directly from iron ore and coal. For most pellet samples, steel can be produced in the bench-scale MW/EAF after 10 minutes microwave heating and 6 minutes electric arc heating.

The start time of electric arcing is greatly affected by the lime content of the pellet sample. The more lime in the pellet sample, the more microwave heating time is required.

The steel yield of the MW/EAF process varies with electric arcing time, coal content, and lime content of pellet sample. Other constituents of the pellet sample and the concurrency of microwave and electric arc heating have little effect on steel yield.

Minimum electric arcing time is needed to achieve high steel yield. For pellet samples with appropriate composition (mainly coal content), steel yield of more than $95 \%$ can be achieved after 6 minutes of electric arc heating.

Steel yield varies with both the coal and lime contents of pellet samples. In order to achieve high yield, the quantity of coal in the pellet sample must guarantee complete reduction of the iron ore. The lime content of pellet sample correlates with steel yield via affecting the extent of steel separation from slag.

\section{Steel and Slag Evaluation}

\section{$\underline{\text { Introduction }}$}

The objective of this study is to evaluate the steel and slag samples obtained from the previously described steelmaking tests, and to find an effective method to produce low sulfur steels in the MW/EAF process.

Slag is a major byproduct in the MW/EAF process. It is formed from the impurities of iron ore, fluxing materials, ash in coal, and refractory materials. In order to generate hot steel with consistent quality, the behavior of molten slag is a major consideration. Molten slag's behavior is dependent on the temperature and composition of the slag. The behavior of slag can be characterized by properties of slag such as viscosity, fluidity, surface tension, and basicity ratio.

Basicity ratio is evaluated through adding together the basic constituents of slag and dividing by the sum of the acid constituents of slag. For slags containing low concentration of $\mathrm{P}_{2} \mathrm{O}_{5}$, the slag basicity ratio can be represented by the mass concentration ratio: $B=(\% \mathrm{CaO}+1.4 \times \% \mathrm{MgO}) /\left(\% \mathrm{SiO}_{2}\right)$. 
In the MW/EAF process, the produced steel contains elements such as carbon, silicon, sulfur and manganese. The steel dissolves some carbon and sulfur because of its intimate contact with coal. Sulfur dissolved in the hot steel reaches equilibrium with sulfur compounds in the slag. If iron ores contain appreciable amounts of calcium phosphate and apatite, the steel also dissolves most of the reduced phosphorus. Other soluble elements of the steel such as silicon and manganese come from reduction of their oxides. Reduction of silica and manganese oxides do not take place until the temperature is above $1490{ }^{\circ} \mathrm{C}$ and $1370{ }^{\circ} \mathrm{C}$ respectively in the presence of carbon. The temperature of slag and steel has an effect on the silicon and manganese content of steel, because the distribution of silicon or manganese between steel and slag is determined by the temperaturedependent equilibrium constant of the reduction reaction.

With a very few exceptions, sulfur is considered undesirable in steel, and there is an increasing demand for steels with lower sulfur levels. Problems associated with sulfur are mainly due to the harmful effects of sulfide inclusions. There are three main methods by which the sulfur content of molten steel may be controlled at a lower level during the melting process. The first method is to mix iron with metallic additions such as magnesium, which in combination with sulfur form very stable sulfides. The second method is to add compound additives such as calcium carbide or soda ash. The third method is to increase the basicity ratio with lime or limestone. Lime will react with sulfur in molten steel to form calcium sulfide in slag. The liquidus isotherms of the ternary system $\mathrm{CaO}-\mathrm{SiO}_{2}-\mathrm{FeO}$ is shown in Figure 1.18. The low melting point areas in pseudo-wollastonite and wollastonite of the diagram are those areas that have the highest sulfur removing capacity.

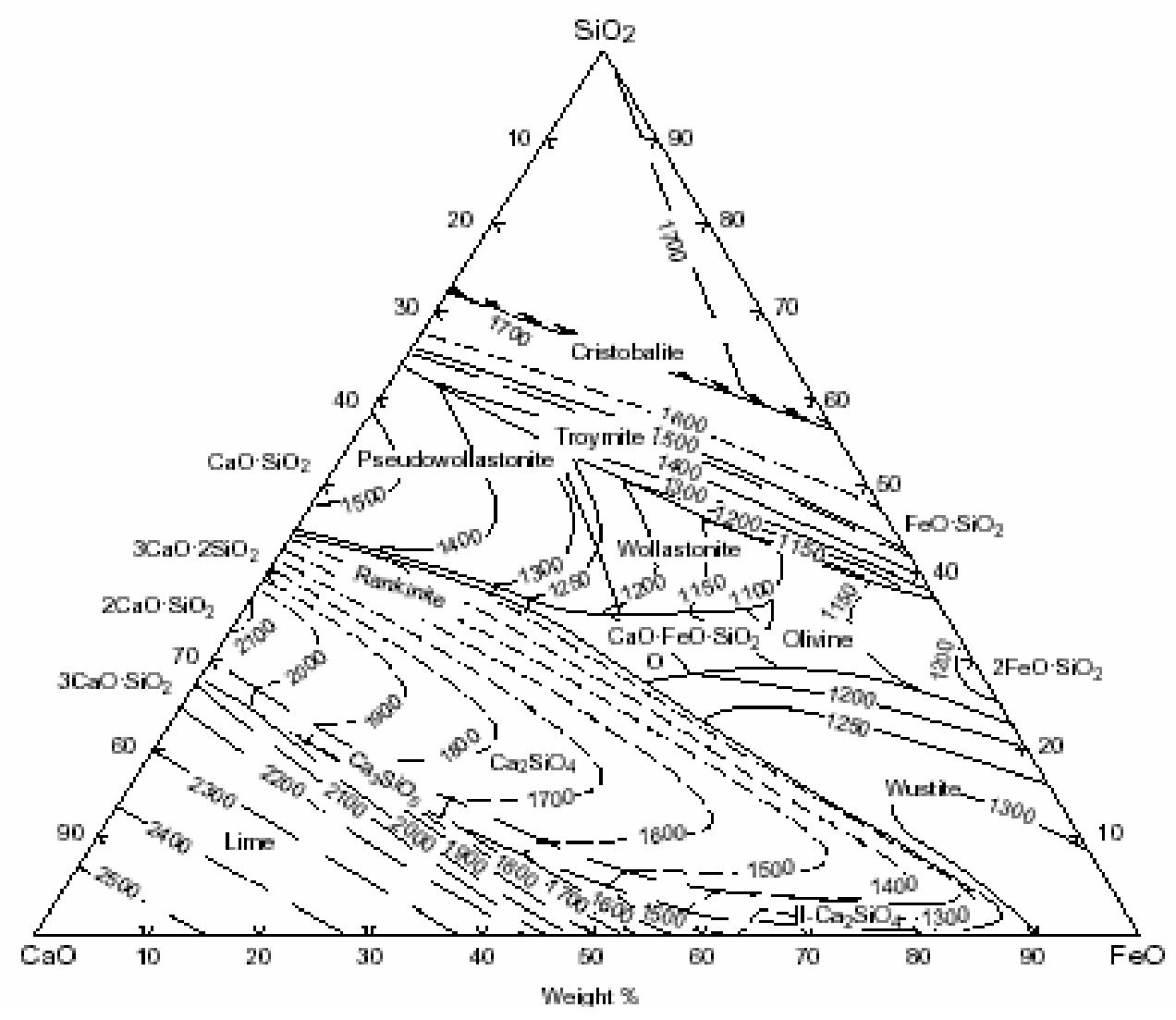

Figure 1.18. Liquidus isotherms of $\mathrm{CaO}-$ $\mathrm{SiO}_{2}-\mathrm{FeO}$ system 
In these experiments for controlling sulfur content of produced steel, only the third method was investigated, and lime was used as the additive. There are many approaches to add lime into slag; some methods are complicated or may have a negative effect on the steelmaking processes. The simple approach is to blend lime into the pellets.

Crucibles are subject to corrosion at elevated temperature, and some oxide components of crucible may be dissolved into slag. Different crucibles interact with slag differently and have different effect on slag composition. Magnesia crucibles have high refractoriness and good resistance to basic slags. Fireclay crucibles mainly consist of silica and alumina that can form some compounds when reacting with lime in slag. Since the melting points of alumina and lime are higher than silica, the compounds such as tridymite and anorthite formed at temperatures below $1500^{\circ} \mathrm{C}$ contain more than $50 \%$ by weight silica.

\section{Experimental Method}

\section{Apparatus}

The carbon and sulfur contents of steel samples were analyzed by using a LECO CS-400 combustion analyzer. Other elements were analyzed using a glow discharge spectrometer (GDS). A CARVER laboratory hydraulic press and SPEX 800 Mixer / Mill were used to crush and pulverize slag. An EDXRF spectrometer (Jordan Valley Ex-6600) was used to analyze the chemical compositions of slag samples.

Materials and Procedures

The compositions of pellet samples and raw materials are the same as listed in Tables 1.7 through 1.10. All steel and slag samples for analysis were obtained from these experiments which were summarized in Appendix B, in which all steel and slag sample names are listed.

\section{$\underline{\text { Steel composition analysis }}$}

Steel samples were analyzed by a commercial lab. The steel samples were polished to remove metal oxides, and the clear surfaces were used for analysis.

\section{Sample preparation for XRD study}

Three slag samples were used for XRD study. Before loading into the XRD equipment, slag samples were crushed and sieved through a No. 100 mesh copper sieve.

\section{$\underline{\text { Slag treatment and composition analysis }}$}

- Crush slag into small chips using a hydraulic press

- Pour all slag chips into steel mixing jar and pulverize for 5 minutes

- Sieve powdered slag through a No. 100 mesh copper sieve

- Fill a XRF sample cup approximately 1/2 full with sieved slag

- Cap XRF sample cup with a piece of polypropylene film and seal it using plastic ring 
- Label sample cup appropriately and place it into XRF spectrometer

- Scan spectrum of sample and standard samples

- Set up analysis procedure using the spectrum of standard samples

- Compute the composition of slag using a defined analysis procedure

\section{$\underline{\text { Results and Discussion }}$}

\section{Steel Composition}

The chemical compositions of steels produced from different pellet samples under different experimental parameters are listed in Table 1.11. The silicon and manganese levels are very low, generally less than $0.01 \%$, the carbon content varies in the range of $0.12 \%-3.35 \%$, and the phosphorus contents are very low, generally around $0.015 \%$. The sulfur contents vary significantly, and the lowest sulfur content of steel is $0.046 \%$.

The measured composition of steel sample tt9 does not represent actual composition of the steel. The measured silicon, titanium and vanadium contents of the steel are higher than that of other steels; it is thought that there is a large slag inclusion in the measured area.

\begin{tabular}{|c|c|c|c|c|c|c|c|c|}
\hline \multicolumn{7}{|c|}{ Table 1.11. Chemical composition of steels (wt \%) } \\
\hline Steel Sample & $\mathbf{C}$ & $\mathbf{S i}$ & $\mathbf{M n}$ & $\mathbf{C r}$ & $\mathbf{V}$ & $\mathbf{S}$ & $\mathbf{P}$ & $\mathbf{T i}$ \\
\hline $\mathbf{t t 1}$ & 2.28 & $<0.01$ & $<0.01$ & 0.08 & 0.25 & 0.7 & 0.011 & $<0.01$ \\
\hline $\mathbf{t t 2}$ & 2.33 & 0.1 & $<0.01$ & 0.13 & 0.42 & 0.64 & 0.009 & 0.09 \\
\hline $\mathbf{t t 3}$ & 2.4 & 0.23 & $<0.01$ & 0.08 & 0.31 & 0.65 & 0.014 & 0.01 \\
\hline $\mathbf{t t 4}$ & 1.08 & $<0.01$ & $<0.01$ & 0.05 & 0.08 & 0.41 & 0.016 & 0.02 \\
\hline $\mathbf{t t 5}$ & 0.6 & $<0.01$ & $<0.01$ & $<0.01$ & $<0.01$ & 0.37 & 0.014 & 0.01 \\
\hline $\mathbf{t t 6}$ & 2.07 & $<0.01$ & $<0.01$ & $<0.01$ & 0.07 & 0.43 & 0.012 & $<0.01$ \\
\hline $\mathbf{t t 7}$ & 0.12 & 0.01 & $<0.01$ & 0.02 & $<0.01$ & 0.44 & 0.01 & $<0.01$ \\
\hline $\mathbf{t t 8}$ & 0.91 & $<0.01$ & $<0.01$ & $<0.01$ & 0.05 & 0.41 & 0.016 & $<0.01$ \\
\hline $\mathbf{t t 9}$ & 3.35 & 2.94 & $<0.01$ & 0.08 & 0.75 & 0.18 & 0.025 & 0.22 \\
\hline $\mathbf{t t 1 0}$ & 1.83 & $<0.01$ & $<0.01$ & 0.01 & 0.12 & 0.37 & 0.013 & $<0.01$ \\
\hline $\mathbf{t t 1 1}$ & 0.65 & $<0.01$ & $<0.01$ & $<0.01$ & 0.13 & 0.4 & 0.012 & $<0.01$ \\
\hline $\mathbf{t d 1}$ & & & & & & & & \\
\hline $\mathbf{t d 3}$ & 0.566 & & & & & 0.156 & & \\
\hline $\mathbf{t d 4}$ & 2.51 & & & & & 0.526 & & \\
\hline $\mathbf{t d 5}$ & 0.427 & & & & & 0.072 & & \\
\hline $\mathbf{t d 6}$ & 1.55 & & & & & 0.0469 & & \\
\hline $\mathbf{t d 7}$ & 1.02 & & & & & 0.469 & & \\
\hline $\mathbf{t d 8}$ & 1.57 & & & & & 0.486 & & \\
\hline $\mathbf{t d 9}$ & 2.32 & & & & & 0.306 & & \\
\hline $\mathbf{t d 1 0}$ & 2.96 & & & & & 0.318 & & \\
\hline
\end{tabular}


The very low silicon and manganese contents of steels indicate that the amounts of silica and manganese oxides reduced in the MW/EAF process are minimums. It is consistent with the facts that the temperature during electric arc heating is about $1350^{\circ} \mathrm{C}-1500^{\circ} \mathrm{C}$, and the temperatures for initializing the reduction reaction of silica and manganese oxides are $1490^{\circ} \mathrm{C}$ and $1370^{\circ} \mathrm{C}$ respectively in the presence of carbon. Low silicon and manganese contents of steel imply that oxide inclusions inside the steels are low. Due to low silicon and manganese contents of hot steel, no effort is needed to remove silicon and manganese via blowing oxygen during steel composition control. The phosphorus level of steels produced by the MW/EAF process is low. This is mainly because the raw materials contain minimal phosphorus compounds.

Slag Composition

The chemical compositions of slags obtained from different pellet samples under different experimental parameters are listed in Table 1.12.

\begin{tabular}{|c|c|c|c|c|c|c|c|c|}
\hline \multicolumn{9}{|c|}{ Table 1.12. Chemical composition of slags } \\
\hline $\begin{array}{c}\text { Slag } \\
\text { Sample }\end{array}$ & $\mathrm{SiO}_{2}$ & $\mathrm{CaO}$ & $\mathrm{TiO}_{2}$ & MnO & FeO & $\mathrm{Al}_{2} \mathbf{O}_{3}$ & $\mathbf{V}_{2} \mathbf{O}_{5}$ & MgO \\
\hline sf1 & 71.82 & 10.062 & 6.8977 & 0.8277 & 3.6631 & 5.0624 & 0.002 & 1.665 \\
\hline sf2 & 67.50 & 10.301 & 7.5172 & 1.0863 & 7.6955 & 4.2202 & 0.002 & 1.681 \\
\hline sf3 & 69.336 & 10.564 & 7.7079 & 1.114 & 5.8988 & 3.5897 & 0.002 & 1.7874 \\
\hline sf4 & 66.957 & 10.004 & 3.789 & 0.429 & 14.085 & 3.0805 & 0.002 & 1.6545 \\
\hline sf5 & 65.635 & 10.629 & 4.0269 & 0.5272 & 13.281 & 4.121 & 0.002 & 1.778 \\
\hline sf6 & 68.363 & 11.238 & 4.2557 & 0.5572 & 9.8919 & 3.8122 & 0.002 & 1.8793 \\
\hline sf7 & 66.135 & 9.3987 & 3.7009 & 0.4539 & 15.147 & 3.429 & 0.002 & 1.7337 \\
\hline sf8 & 67.343 & 9.7843 & 3.8504 & 0.4116 & 13.005 & 3.4211 & 0.002 & 2.1829 \\
\hline sf9 & 64.194 & 25.099 & 2.751 & 0.4663 & 4.8182 & 2.6701 & 0.002 & 1.544 \\
\hline sf10 & 65.987 & 25.372 & 2.1318 & 0.2572 & 2.9426 & 3.3075 & 0.002 & 1.6098 \\
\hline sf11 & 63.78 & 19.331 & 4.3952 & 1.2178 & 2.3426 & 5.8533 & 0.002 & 3.0768 \\
\hline sd1 & 40.905 & 40.906 & 1.8943 & 0.709 & 9.5618 & 3.38 & 0.002 & 2.6359 \\
\hline sd3 & 42.416 & 46.31 & 2.5667 & 0.5188 & 3.3657 & 3.0761 & 0.002 & 1.7441 \\
\hline sd4 & 67.605 & 20.252 & 2.7342 & 0.4506 & 3.1068 & 1.923 & 0.002 & 3.9216 \\
\hline sd5 & 38.629 & 44.725 & 1.813 & 0.9377 & 9.6628 & 0.8187 & 0.002 & 3.4094 \\
\hline sd6 & 38.27 & 44.365 & 5.5944 & 1.1577 & 4.1715 & 2.9705 & 0.002 & 3.4664 \\
\hline sd7 & 54.034 & 31.334 & 4.2549 & 0.7529 & 1.3392 & 5.4964 & 0.002 & 2.785 \\
\hline sd8 & 50.918 & 34.666 & 1.979 & 0.6567 & 3.1147 & 5.5108 & 0.002 & 3.1508 \\
\hline sd9 & 47.631 & 29.51 & 4.4842 & 0.6375 & 2.8286 & 4.7509 & 0.002 & 10.155 \\
\hline sd10 & 49.936 & 32.088 & 3.1404 & 0.8548 & 1.0779 & 3.6481 & 0.002 & 9.2526 \\
\hline
\end{tabular}

Table 1.12 illustrates that slag composition varies significantly and the major constituents of most slags are $\mathrm{SiO}_{2}$ and $\mathrm{CaO}$. Some slags contain a large quantity of unreduced iron ore. Some contain a large quantity of magnesia due to magnesia in the crucible lining material. Compared 
with BF slags, the alumina level of these slag samples is lower, because the fluxing material for the MW/EAF process is mainly lime.

Figure 1.19 exhibits two diffractograms of slags. The slags contained a high glass phase content. Some unreduced iron ores in the slags were transformed into pseudo-brookite $\left(\mathrm{Fe}_{2} \mathrm{TiO}_{5}\right)$ and some still existed as iron oxide.
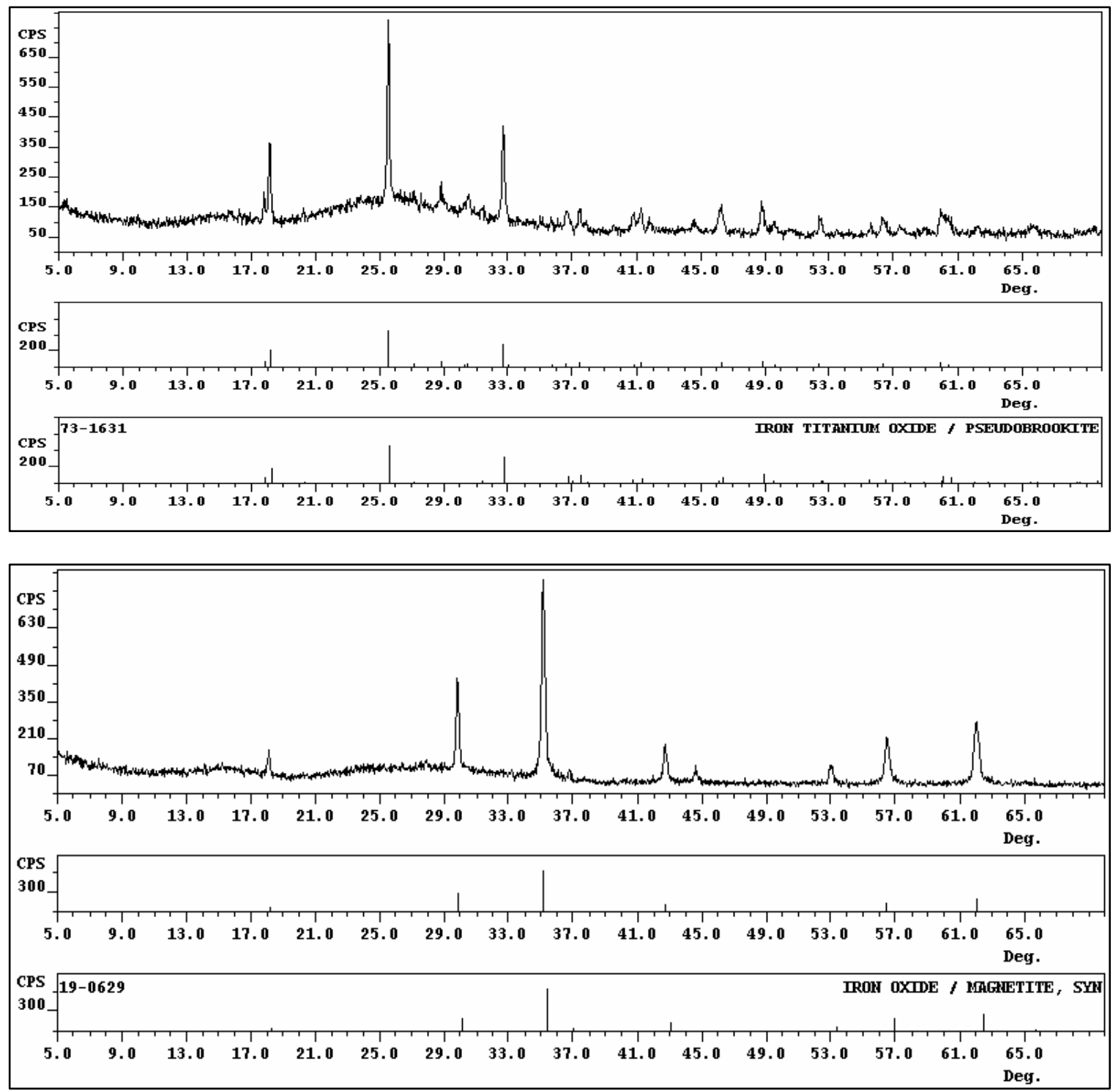

Figure 1.19. XRD spectrums of low steel yield slag

A broad background feature on the baseline displays a high content of glass phase. The first spectrum in Figure 1.19 indicates that the slag contained a crystalline phase component pseudobrookite $\left(\mathrm{Fe}_{2} \mathrm{TiO}_{5}\right)$. The second spectrum in Figure 1.19 indicates that the slag contained magnetite; this crystalline phase component is thought to be unreduced iron ore. 


\section{Effect of Coal Ratio on Carbon Content of Steel}

Figure 1.20 illustrates the variation of the carbon content of the steel samples with respect to the coal to magnetite ratios of pellet samples. The steels produced from high coal pellets have higher carbon content than that of steels produced from lower coal pellets. The minimum carbon content and the average carbon content of steels, with a steel yield over $95 \%$, are $0.427 \%$ and $1.49 \%$ respectively.

Figure 1.20 also indicates a wide variation of carbon content of steels produced from the same pellet samples. It is an indication of unstable furnace operations. Different operators may generate very different steelmaking results.

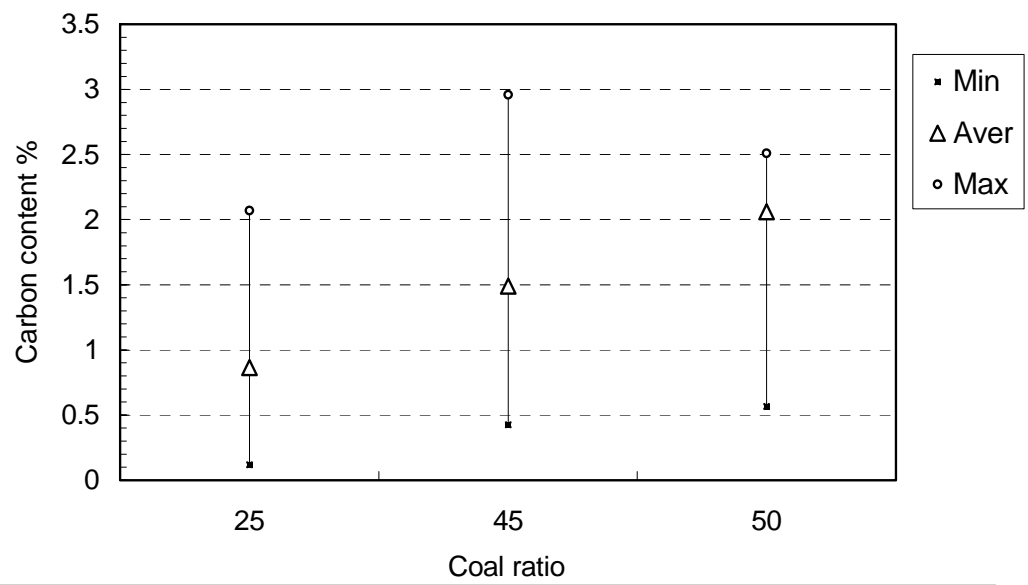

Figure 1.20. Variation of carbon content with pellet coal ratio

\section{Factors Effecting Sulfur Content of Steel}

Figure 1.21 illustrates the effect of slag's basicity ratio on the sulfur content of steel. The average sulfur content of the steel decreases as the slag basicity ratio increases. When the slag basicity ratio was between $1.0-1.3$, both the average sulfur content of the steels and the maximum sulfur content of the steel produced were the lowest among the three groups of steels.

In these experiments, slag basicity is mainly dependent on the limemagnetite ratio of the pellet sample, crucible used, and crucible lining materials. All slag with a basicity ratio greater than 1.0 was actually generated using magnesia crucibles, as illustrated by Figure 1.22 .

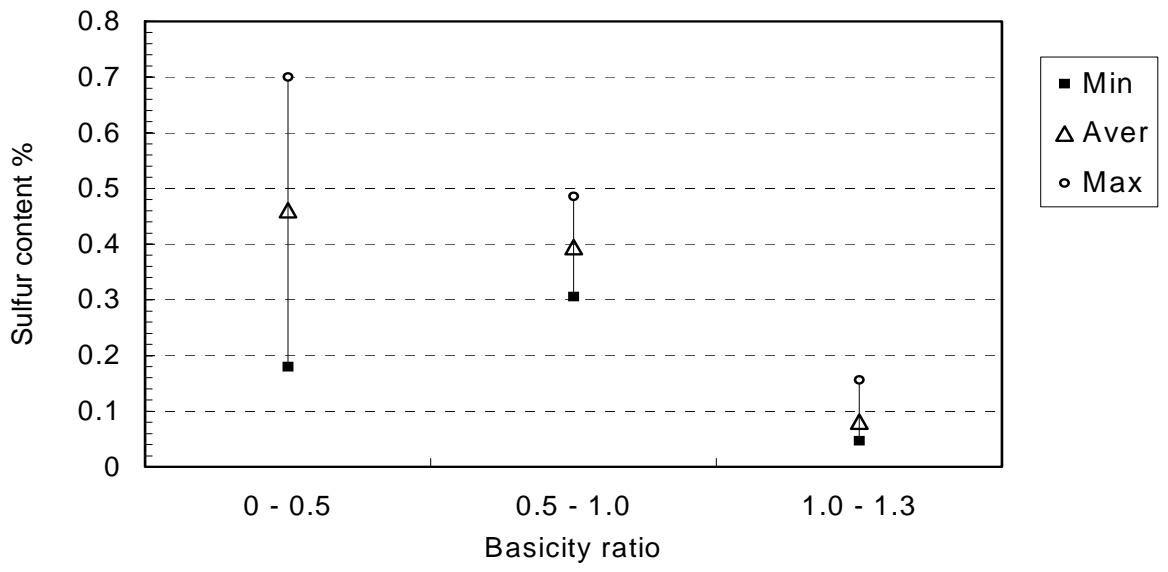

Figure 1.21. Effect of basicity ratio on sulfur content of steel

Figure 1.22 illustrates the effects of crucible and lining on the sulfur content of steel. The sulfur content of the steel samples can be significantly reduced by using magnesia crucibles in combination with lime lining. Steel with minimum sulfur content of $0.047 \%$ were achieved by using magnesia crucibles and lime lining. The sulfur content of steels produced by fireclay crucibles with magnesia lining is a little lower than that of steels produced by fireclay crucibles with lime lining. 


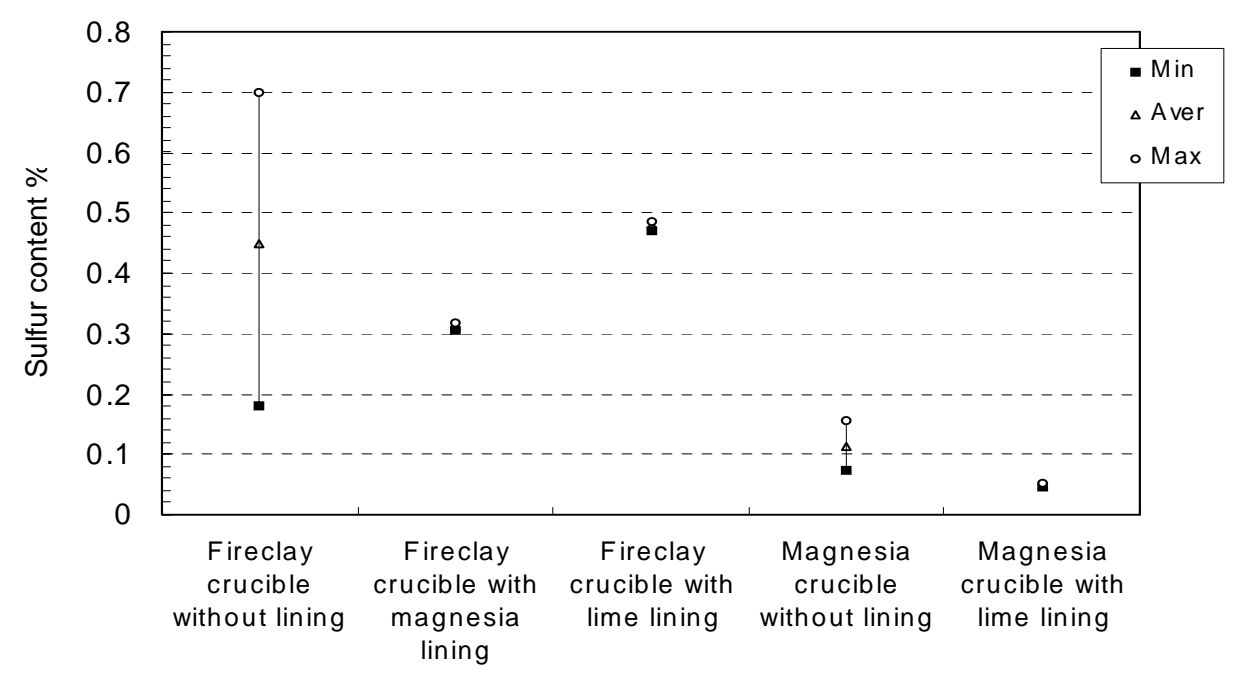

Figure 1.22. Effects of crucible and lining on sulfur content of steel
The average sulfur content of steels produced using fireclay crucibles is nine times higher than that of steels produced when using magnesia crucibles with lining, and is four times higher than that of steels produced by using magnesia crucibles. The change in the basicity ratio of

slag due to the interaction between crucible, lining materials and slag results in the significant differences. More information was determined after post-mortem analysis of fireclay crucibles. Fireclay crucibles, even when coated with lining, were severely corroded and most of the interior of the crucible dissolved into slag. But the magnesia crucibles were not corroded. The lower portion of the lime linings coating fireclay crucibles were totally dissolved into slag, but the low portion of lime linings coated on magnesia crucibles were only partially dissolved into slag. The dissolved crucible or lining materials definitely changes the basicity ratio of slag, so crucible and lining composition are important factors for producing steels with lower sulfur levels.

\section{Slag Basicity}

Figure 1.23 illustrates the variation of the slag basicity ratio with the lime-magnetite ratio of pellet samples when using fireclay crucibles. Figure 1.23 indicates that the basicity of slags generated in fireclay crucibles by pellet samples with a limemagnetite ratio less than 35/100 were below 0.4; Pellet samples with high lime-magnetite ratios usually generated slag with high basicity.

Due to the reaction with lime in slag, the fireclay crucible interior eroded badly and dissolved into slag. Assuming that no materials from the crucible are dissolved, the theoretical basicity of slag

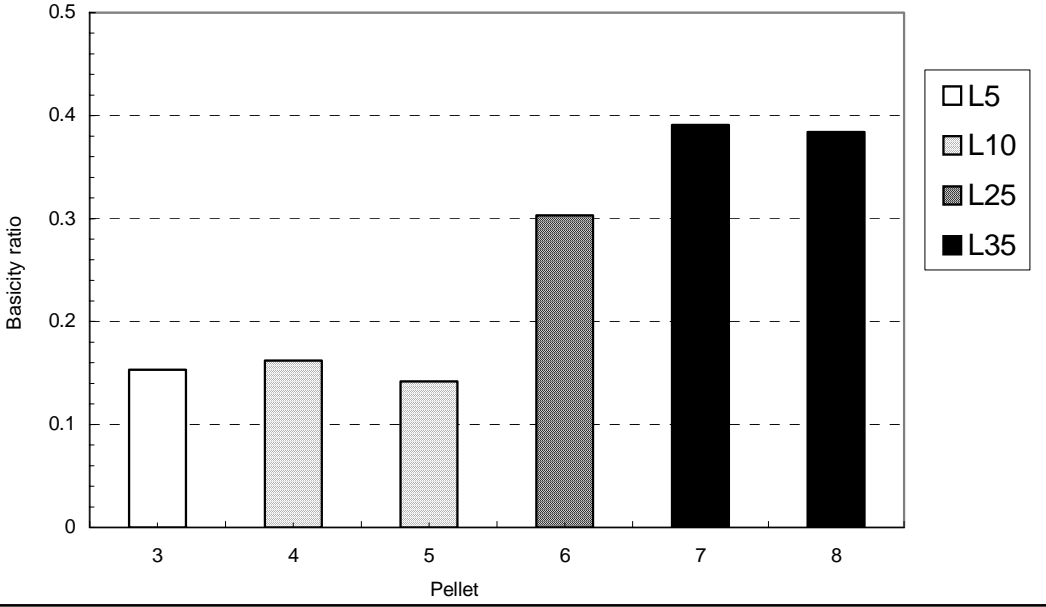
formed by pellet sample \#6 is

Figure 1.23. Variation of slag basicity ratio with lime ratio of pellet sample when using fireclay crucible 
2.2. Based on the temperature of slag and the theoretical basicity of slag, it can be deduced that the major proportion of the dissolved oxides is acid oxide silica. So simply increasing the lime ratio of pellet samples is not an effective method for increasing the basicity of slags in fireclay crucibles.

Figure 1.24 illustrates the basicity ratios of some slag generated in fireclay crucibles coated with different lining materials. No significant difference between lime and magnesia linings was observed, and lining can increase the basicity ratio of slag formed in fireclay crucibles.

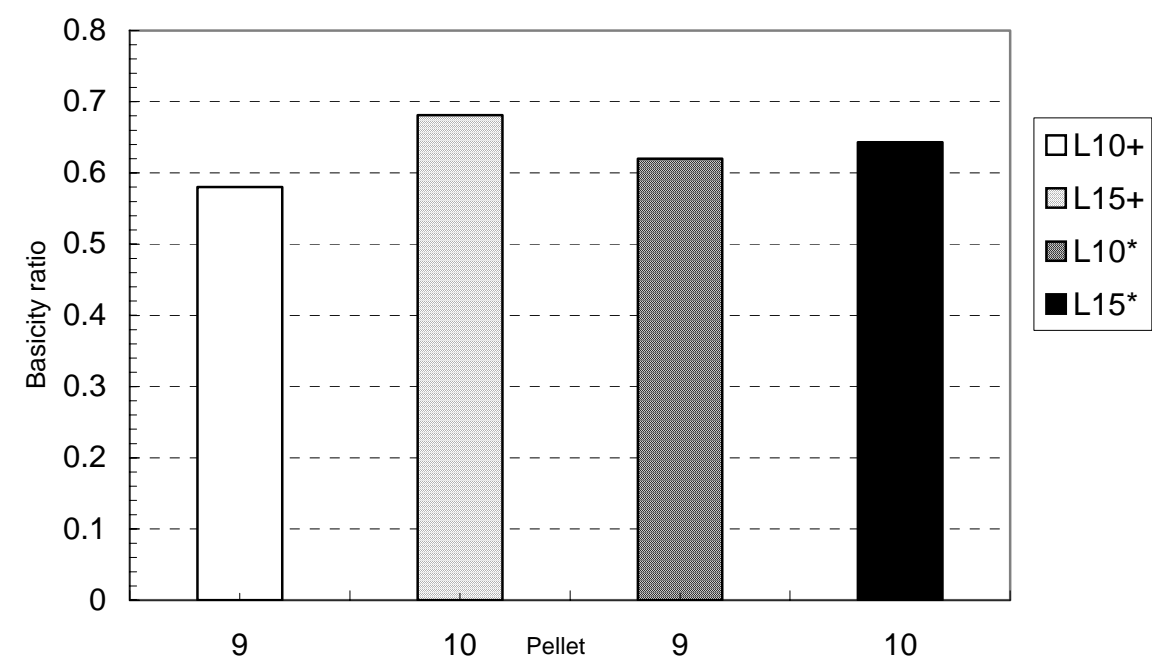

Figure 1.24. Basicity ratio of slag generated in fireclay crucible with lining Where + indicates lime lining, and * indicates magnesia lining
The slags generated from pellet samples with a lime ratio $10 / 100$ or $15 / 100$ in coated fireclay crucibles had basicity ratios in the range of 0.55 - 0.7. Comparing with the basicity ratios of the slags in Figure 1.23 , the increase of slag basicity ratios by using linings was considerable. Lining layers of about 0.5 inches thick had a small temperature gradient, so it could not lessen the extent of crucible corrosion by hot slags, and the increase of slag basicity came from the solution of lime or magnesia into the slag.

Figure 1.25 illustrates the basicity ratios of slags generated in magnesia crucibles with or without lime lining. The basicity ratios of the slags generated in magnesia crucibles were generally higher than that of slags generated in fireclay crucibles and were in the range of $1-1.2$. Lime lining could increase the slag basicity ratio about 0.05 0.15. The increase of slag basicity due to lime lining was dependent on pellet samples. The less the lime ratio of pellet samples, the more

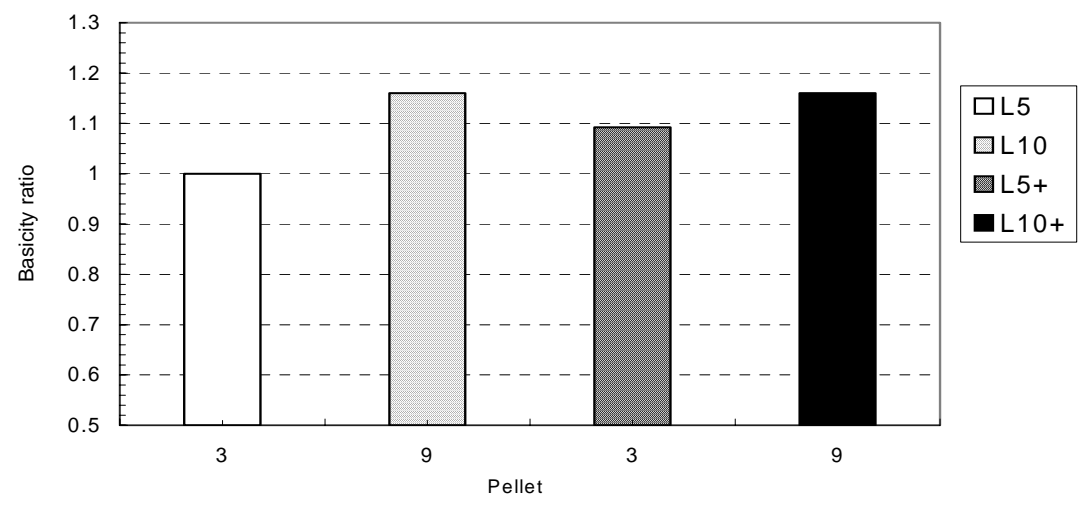

Figure 1.25. Basicity ratios of slag generated in magnesia crucible with or without lining. Where symbol + means lime as lining increase in the basicity ratio of the generated slag. 
As illustrated by the liquidus isotherms of ternary system $\mathrm{CaO}-\mathrm{SiO}_{2}-\mathrm{FeO}$, molten slag with very low iron oxide content and high content of molten lime mainly consists of compounds $3 \mathrm{CaO}-\mathrm{SiO}_{2}, 2 \mathrm{CaO}-\mathrm{SiO}_{2}$ and $3 \mathrm{CaO}-2 \mathrm{SiO}_{2}$. These compounds have a basicity ratio in the range of $1.3-2.3$, and their melting points are above $1500{ }^{\circ} \mathrm{C}$. So the basicity ratio of slag generated by using a magnesia crucible with lime lining at temperatures below $1500{ }^{\circ} \mathrm{C}$ had already reached the maximum possible value.

\section{$\underline{\text { Conclusions }}$}

Most of the steels produced using the MW/EAF process had silicon and manganese levels less than $0.01 \%$ and an average phosphorus level of $0.015 \%$. The carbon levels of the steels were in the range of $0.12 \%-3.35 \%$.

The minimum carbon content and the average carbon content of produced steels were $0.472 \%$ and $1.49 \%$. The steel carbon content correlates with the coal ratio of pellet samples. The minimum carbon content and the average carbon content of steels produced from pellet samples with a high coal ratio generally are higher than that of steels generated from pellet samples with a low coal ratio.

The sulfur content of steel correlates with the slag basicity. When slag basicity is in the range of $1.0-1.3$, both the average sulfur content of steels and the minimum sulfur content of steel are the lowest. The lowest sulfur content of steel produced from the MW/EAF steelmaking tests is $0.0469 \%$.

Slag basicity is dependent on the lime ratio of the pellet sample, the type of crucible and lining material. Slag basicity increased with the increase of the lime ratio of the pellet sample. Crucible lining can increase slag basicity to some extent. Lime is a slightly better lining material than magnesia for obtaining both high basic slags and steels with low sulfur level.

Slag with high basicity can not be obtained using fireclay crucibles, even though the lime ratio of the pellet sample is as high as 35/100 and different lining materials are used. In order to obtain slag with basicity in the range of $1.0-1.3$, magnesia crucibles must be used and the lime ratio of the pellet sample can be as low as 10/100.

All steels with lower sulfur levels (around 0.05\%) were obtained using magnesia crucibles with lime as lining and pellet samples with a lime ratio of $5 / 100$ or $10 / 100$. Consequently, it is unnecessary to use pellet samples with very high lime ratios in order to produce low sulfur steel, if magnesia crucibles with lime lining are used. 


\subsection{Iron Ore Reduction by Microwave Assisted Hybrid Heating}

Experiments conducted in baseline microwave steelmaking and bench-scale MW/EAF steelmaking have shown that the majority of the volatiles in coal do not participate in iron ore reduction or iron ore heating in an open reactor. The volatiles come out of the iron ore pellet mass before iron ore reduction starts. In addition, excessive fixed carbon in iron ore pellets reacts with oxygen to form $\mathrm{CO}$, which is also released from the iron ore pellet mass. Both the volatiles and CO contain a great amount of chemical and thermal energy. These gases burned above the iron ore pellet mass in our experiments and had no contribution to the heating of iron ore pellets. This chemical and thermal energy must be utilized. One way of utilization is to preheat iron ore. If successful, the energy utilization can reduce the microwave power requirement. The hybrid heating concept could be further extended to conventional heating equipment such as conventional DRI production. An addition of microwave heating to conventional heating could greatly improve heat transfer to thick mass materials. This experiment was designed to provide some preliminary results.

\section{Experimental Method}

\section{$\underline{\text { Apparatus }}$}

The experiments were conducted using a self-made thermo-gravimeter shown in Figures 1.26 to 1.28. Figure 1.29 shows a schematic diagram of the apparatus. A mullite pipe $(\mathrm{OD}=2$ ", $\mathrm{ID}=1.97$ ”, $\mathrm{L}=48$ ") is put through the microwave oven (GE RVM1335, 900W). The pipe, which lies in the cavity of the microwave, is covered by electrical heating elements $(2400 \mathrm{~W})$; the remainder of the cavity is filled with alumina oxide bricks and fiber glass for heat insulation. A stainless steel sheathed thermocouple is inserted through the top of the pipe for monitoring and controlling the temperature in the reactor.

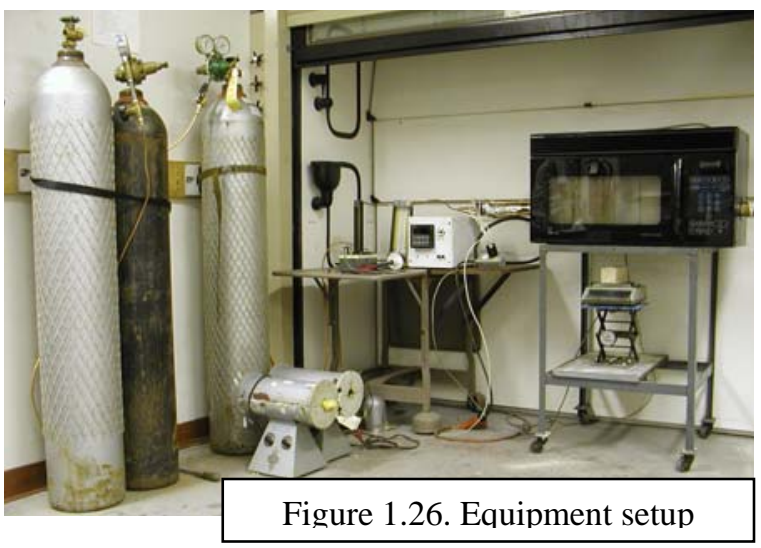

One end of the mullite pipe is connected to the outlet of the

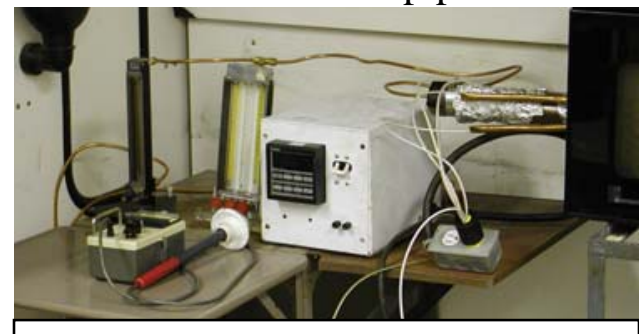

Figure 1.27. Equipment control (temperature controller, flow meter, microwave detector) gas cylinder, and the flow rate of the gas is controlled by the flow meter. The other end of the mullite pipe is open to the air for the release of the reaction and

product gases. The entire reactor system is installed in a fume hood in order to minimize exposure to potentially noxious gases.

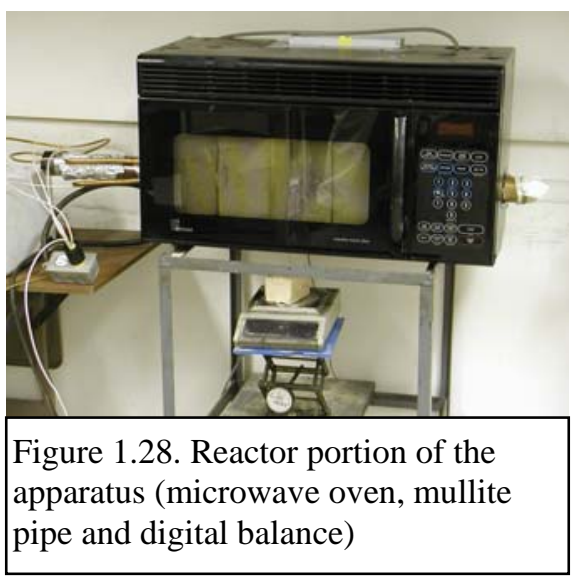

Figure 1.28. Reactor portion of the pipe and digital balance) 


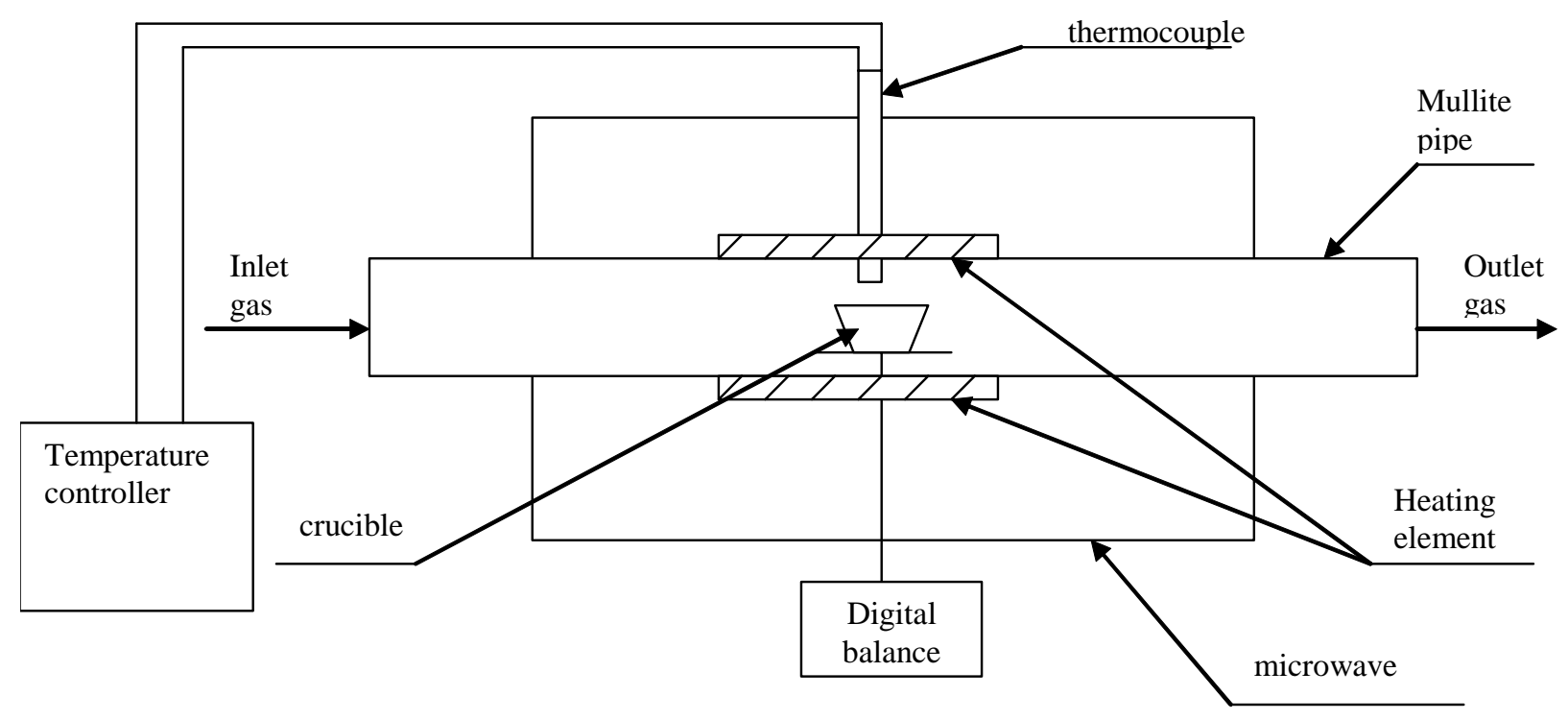

Figure 1.29. Schematic diagram of hybrid heating apparatus

According to experiments done by J.A. Aguilar, temperature information provided by either stainless sheathed thermocouples or optical pyrometers are in good mutual agreement. The disadvantage of an optical pyrometer is that it can only measure the surface temperature, while the disadvantage of a thermocouple is that it is difficult to obtain a readable signal (values on the display are changing too fast when microwaves are applied). Also according to J.A. Aguilar, high purity aluminum oxide crucibles will not affect reducibility. In our system, we used aluminum oxide crucibles and stainless steel sheathed thermocouples to address these factors.

\section{$\underline{\text { Materials }}$}

Pure synthesized $\mathrm{Fe}_{3} \mathrm{O}_{4}$ powder provided by Harcros Pigments Inc. (11 Executive Dr., Suite 1 Fairview Heights, IL 62208) was used in this study.

\section{Reducing Agents}

Volatiles consist of hydrocarbons and can be reformed into $\mathrm{H}_{2}$ and $\mathrm{CO}$. Both gases are good iron ore reducing agents. In this study, both gases were used, either singly or as a mixed gas, as the reducing agent. Ar and $\mathrm{N}_{2}$ were used to dilute both gases. These were selected to protect the reduced iron from being re-oxidized because the reduced iron in this system is highly reactive and can be easily re-oxidized.

\section{Experimental Procedure}

- Weigh the sample using the digital balance

- Turn on the hood 
- Add sample in the crucible

- Put the crucible charged with sample on the crucible holder in the mullite pipe

- Adjust the balance

- Connect one end of the mullite pipe to the flow meter

- Turn on the Argon or Nitrogen, adjust the gas flow rate and let the gas flow for 15 minutes to purge the oxygen from the mullite pipe

- Turn on the reducing gas, adjust the flow rate and let the reducing gas flow for 10 minutes to make sure the concentration in the mullite pipe is uniform

- Turn on the power of the conventional heating or microwave heating, and record the time, temperature, and balance data.

- After the experiment, analyze the sample.

\section{Results and Discussion}

Four different experiments were conducted to evaluate the following scenarios: 1) reduce magnetite by conventional heating held at $500^{\circ} \mathrm{C} ; 2$ ) reduce magnetite by conventional heating held at $1000^{\circ} \mathrm{C}$; 3) preheat magnetite by conventional heating up to $500^{\circ} \mathrm{C}$ and then turn on microwave heating and shut off conventional heating; and 4) heat magnetite with conventional and microwave heating simultaneously from ambient temperature to $500^{\circ} \mathrm{C}$ and then continue microwave heating and shut off conventional heating. A mixture of $\mathrm{Ar}$ and $\mathrm{H}_{2}$ was used as the reducing agent. The gas flow rates were the same for all four tests. The weight loss of the magnetite sample over time was recorded. Reduction degree is defined as the degree of oxygen atom loss in iron oxide. Using the weight loss of the magnetite sample, reduction degree can be calculated. $\mathrm{H}_{2}$ is known as a better reducing agent than $\mathrm{CO}$ for iron ore due to lower reduction temperature, around $500^{\circ} \mathrm{C}$, and higher diffusion rate. Several existing DRI processes such as fluidbed DRI process use hot hydrogen as both a heating source and reducing agent.

The experimental data is plotted in Figure 1.30. We can see that microwave assisted heating dramatically decreased the reduction time of conventional low temperature reduction. And, in comparison with conventional high temperature reduction, microwave assisted heating further shortens reduction time. Microwave assisted heating can be combined with conventional heating in two ways: concurrent heating or conventional preheating followed by microwave heating. Concurrent heating can enhance existing heating equipment with the addition of microwave heating. A second option, hybrid heating, could utilize off-gases for pre-heating, followed by microwave heating.

Reforming volatiles will generate a mixture of $\mathrm{CO}$ and $\mathrm{H}_{2}$. It has been reported that a mixture of $\mathrm{CO}$ and $\mathrm{H}_{2}$ at a ratio of $3: 1$ is a better

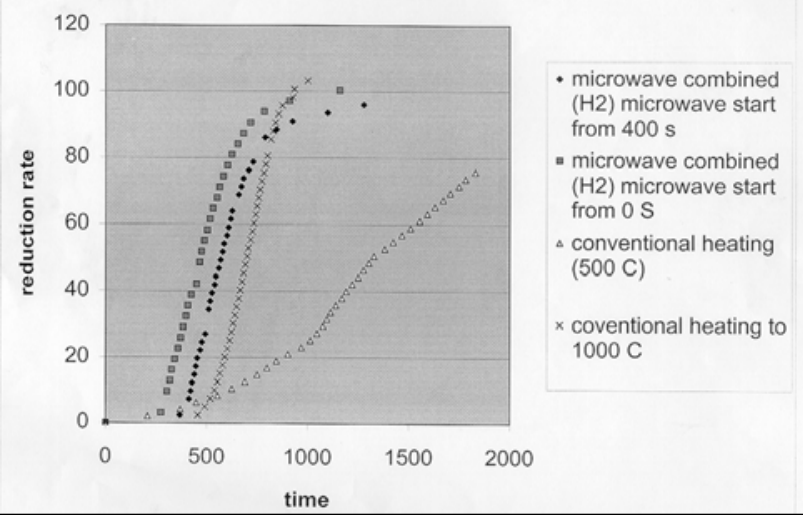

Figure 1.30. Comparison of conventional and microwave assisted magnetite reduction reducing agent than either $\mathrm{CO}$ or $\mathrm{H}_{2}$ because reduction by $\mathrm{CO}$ is weakly exothermic and reduction by $\mathrm{H}_{2}$ is strongly endothermic. Using the gas mixture, reduction takes place with 
almost no release of heat. Figure 1.31 compares using either $\mathrm{CO}$ or $\mathrm{H}_{2}$ as the reducing agent or two mixtures of $\mathrm{CO}$ and $\mathrm{H}_{2}$ as the reducing agent for microwave assisted magnetite reduction. A mixture of $\mathrm{CO}$ and $\mathrm{H}_{2}$ as the reducing agent can significantly decrease reduction time under microwave irradiation conditions.

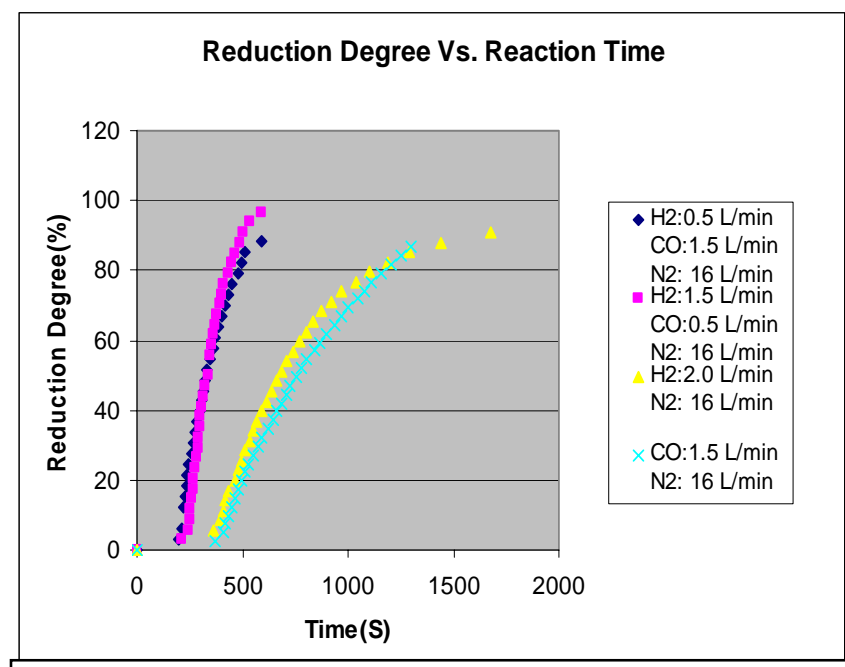

Figure 1.31. Reduction degree versus time (start microwave after conventional heating up to $773 \mathrm{~K}$ )

During our experiments, we found that there is an abrupt increase of temperature in microwave combined heating. In order to investigate this phenomenon, we ran the test without any iron oxide sample in the system. The experimental result is shown in Figure 1.32. The temperature increases from $773^{\circ} \mathrm{K}$ to $1073^{\circ} \mathrm{K}$ in less than 10 seconds. Then the temperature remains stable around $1073^{\circ} \mathrm{K}$. This temperature is the gas temperature due to the microwave induced plasma. Several experiments were repeated to verify the phenomena and the results are also shown in Figure 1.32. We found that only a reaction system where hydrogen exists can generate microwave induced plasma, once the hydrogen was turned off, the microwave induced plasma disappeared. This phenomenon is in agreement with data reported in the literature. The source of additional power corresponding to the elevated temperatures may be an energetic reaction of atomic hydrogen caused by a resonance energy transfer between hydrogen atoms and $\mathrm{Ar}+$.

According to R.L. Mills, the average electron temperature Te for the ArgonHydrogen was high, $11,600 \pm 5 \%{ }^{\circ} \mathrm{K}$. This high electron temperature may be another reason why microwave combined heating can increase the reduction rate of iron oxide.

We conducted a similar test, changing $\mathrm{H}_{2}$ to $\mathrm{CO}$. As shown in Figure 1.33, the phenomenon is the same as the test done using $\mathrm{H}_{2}$. The test was repeated, changing the inert gas from argon to nitrogen. From the experiment result shown in Figure 1.34, we can see that

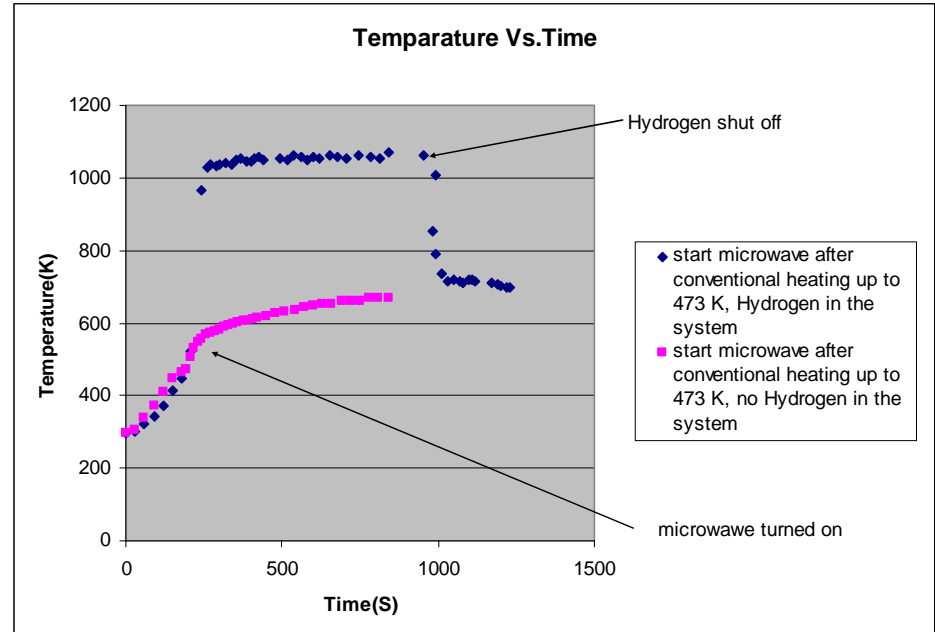

Figure 1.32. Temperature versus time (Ar: $\left.10 \mathrm{~L} / \mathrm{min}, \mathrm{H}_{2}: 2 \mathrm{~L} / \mathrm{min}\right)$ microwave induced plasma also occurred in the $\mathrm{N}_{2}$ system.

The initial temperature at which the microwave is turn on plays an important role in the generation of microwave induced plasma. Only when the initial temperature reaches a certain 
point does microwave induced plasma occur, as shown in Figure 1.34. There appears to be a threshold temperature triggering the microwave induced plasma. Of course, for different reducing gas, different inert gas, and different flow rate, the threshold temperature should be different. In the future, for a specific system, the critical threshold temperature should be found and optimized.
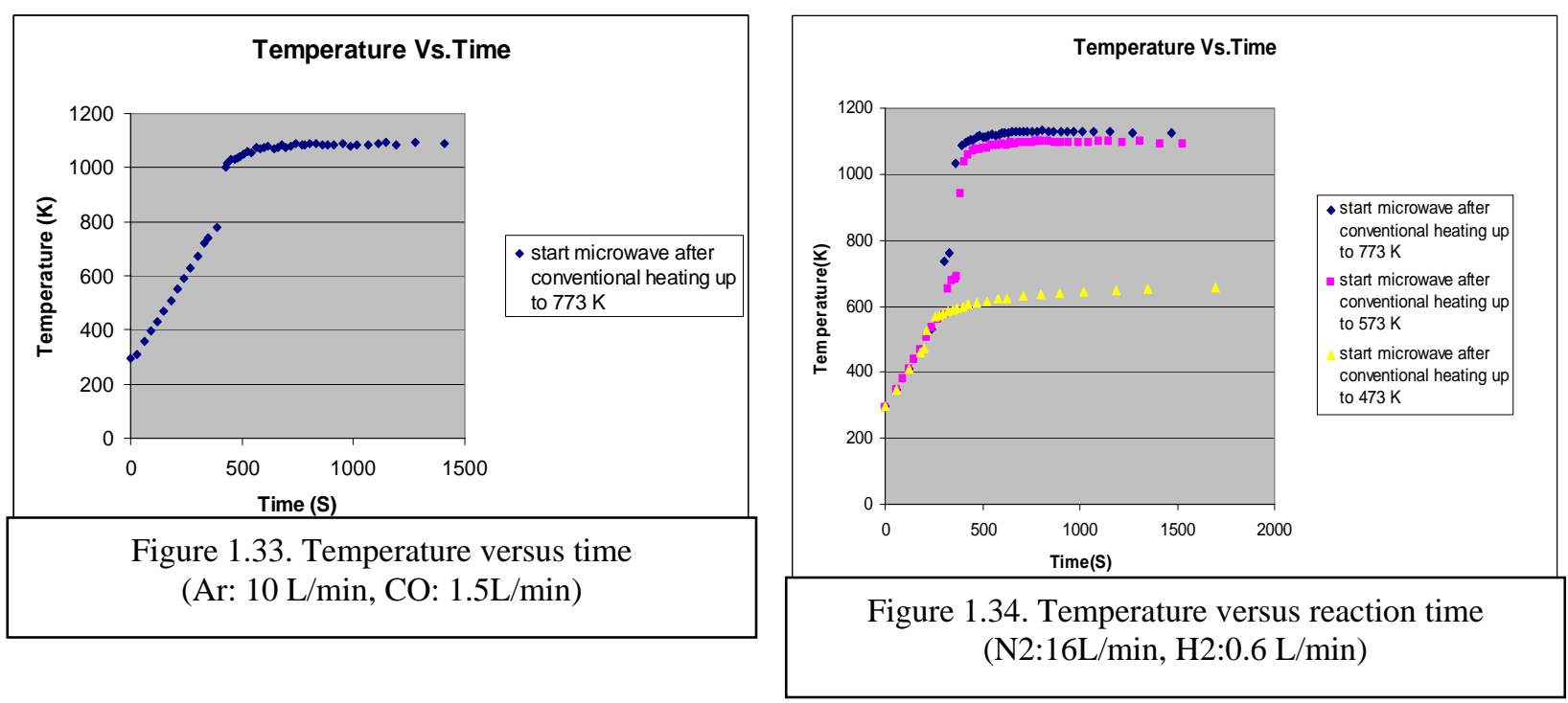

\section{Conclusions}

In comparison with conventional heating, microwave assisted hybrid heating can significantly reduce iron oxide reduction time.

A mixture of $\mathrm{H}_{2}$ and $\mathrm{CO}$ is a better reducing agent than either $\mathrm{H}_{2}$ or $\mathrm{CO}$ alone as the reducing gas for iron ore reduction under microwave assisted heating. The mixture can significantly reduce reduction time.

Microwave assisted heating can have a "temperature jump" phenomenon in both Ar and $\mathrm{N}_{2}$ atmosphere. The generation of the "temperature jump" phenomenon depends on the reducing gas flow rate, the temperature at which microwave irradiation starts, and the inert gas. In general, at the same flow rate of reducing gas and by using the same inert gas, it is easier to find the "temperature jump" phenomenon when microwave irradiation starts at a higher temperature. 


\section{Task 2. Potential Applications and Flowsheet Development}

During the execution of this project, we conducted many experiments, collected and reviewed related literature, and discussed the MW assisted steelmaking technology internally and externally. We have envisioned various alternative scenarios in which MW energy could be utilized for steelmaking. These alternatives have both advantages and disadvantages in one way or another. It is premature to conclude which one is the best and rule out the others. These alternatives are presented and discussed in this section.

A flowsheet represents activities and subsequent flow pattern of input and output of a process. The activities are usually discrete in purpose and are performed using certain types of equipment. The flow patterns of input and outputs have certain characteristics such as phase, composition, and volume that have to be addressed relative to the process's main purpose. Thus the flowsheet represents a visual illustration that defines some technical, economic and environmentally based logic to accomplish the main purpose. With still limited knowledge of MW assisted steelmaking, we can only propose conceptual flowsheets.

It is possible to utilize MW energy to assist steelmaking in several alternatives. From the operation point of view, these alternatives could be divided into batch and continuous operations. From the equipment point of view, these alternatives could be divided into MW/EAF integrated units and MW/EAF separated units. From the product point of view, these alternatives could be divided into direct steelmaking and DRI production. To facilitate discussions, we distinguish these alternatives in six groups: 1) direct steelmaking by batch operation, 2) direct steelmaking by continuous operation, 3) MW/EAF integrated units, 4) MW/EAF separated units, 5) MW assisted DRI production, and 6) MW only DRI production.

Each alternative implies significant impact on equipment requirements, production line and management, and has associated advantages and disadvantages. The concept of MW assisted steelmaking can be considered as a revolutionary change to the current steelmaking processes. To reduce obstacles of executing this new technology, it seems best to maximize utilization of existing equipment and technologies, or in the other words, combine MW heating with existing equipment and technologies to achieve the maximum economic benefit. The discussions on possible alternatives follow.

\section{1) Direct Steelmaking by Batch Operation}

This alternative is to produce molten steel directly from a shippable agglomerate consisting of iron oxide concentrate, coal, and fluxing agent without the intermediate steps of coking, sintering, BF ironmaking, and BOF steelmaking. To achieve this goal, a commonly used EAF furnace could be modified with the introduction of microwaves into the chamber through a single or multiple waveguides or through multiple windows if MW generators are mounted on the wall of the furnace chamber. The viability of this alternative relies on rapid (30 minutes or less) iron ore reduction in the furnace by MW irradiation. The cycle time of each batch operation including raw material charging, MW heating, EA heating, slag discharge, refining, and molten steel discharge is less than 60 minutes. 
In this alternative, iron ore is crushed, ground, and concentrated by conventional processing. The concentrated iron oxide is mixed with pulverized coal and limestone, and then agglomerated at ambient temperature for shipping strength. The coal serves as a reducing agent for iron oxides and as an auxiliary heat source via exothermal reaction in the presence of oxygen. Limestone is used as the fluxing agent.

The potential application of this technology is to modify EAFs with an auxiliary microwave heating system as shown in Figure 2.1. A port (101) is created in the cover (102) of a conventional EAF to introduce microwaves (103) into the chamber (104) through a waveguide (105). A charge of iron oxide/coal/limestone (ICL) agglomerate (106) is loaded into the chamber. Microwave energy is introduced through the waveguide, where the agglomerates absorb microwave energy and their temperature rises to the point of coal ignition.

Exothermal heat from the carbon/oxygen reaction (107) is generated to further increase temperature. The iron oxide then

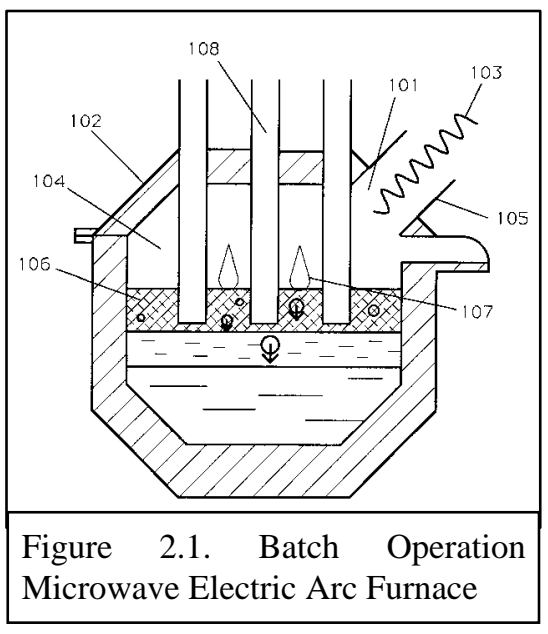
reacts with the reductant to become directly reduced iron. The EAF electrodes (108) then descend to provide electric arcing energy to the material, producing molten steel and slag. The molten slag and steel are removed by conventional methods utilizing the tilting of the furnace chamber. Thus the furnace can use feed ranging from 100\% scrap to 100\% ICL agglomerates.

This alternative requires intensified MW input to the chamber in order to achieve very quick iron ore reduction. It is a challenge for the current MW equipment manufacturers to manufacture an integrated high power MW generator at low cost. Multiple small MW generators could be a solution to this problem.

The advantages of this alternative include minimum reduction needed, to the degree of being a good electric conductor, continuous reduction by MW and EAF heating, no reoxidation, and a single furnace for both reduction and smelting. The disadvantages include difficulty of MW seal and difficulty of residual heat utilization.

\section{2) Direct Steelmaking by Continuous Operation}

The second alternative is to design and build a submerged arc electric furnace which can be operated continuously as depicted in Figure 2.2. The furnace chamber will be stationary and corporate the steps of the first alternative, but in continuous fashion, utilizing feed chute (201) and tap holes for steel (202) and slag (203) discharge. The design will incorporate issues of geometry, timing, and durability of materials of construction. During operation, the raw materials are continuously fed into the

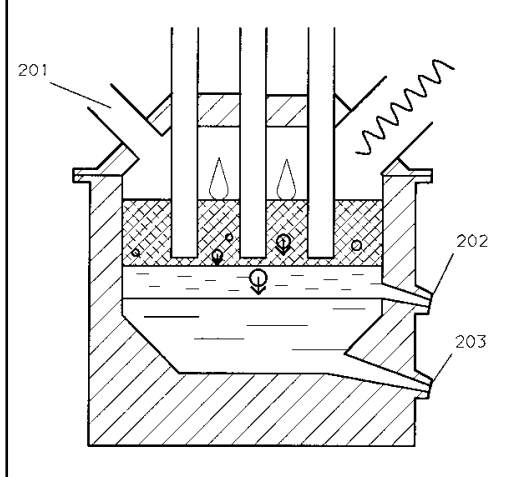

Figure 2.2. Continuous Operation Microwave Electric Arc Furnace chamber and molten steel and slag accumulate on the bottom of furnace. After molten steel or slag reaches a certain level, it will be discharged through its tap hole. The tap hole will be 
blocked after completion of discharging until the next cycle. This continuous operation is similar to the continuous operation of BF.

Direct steelmaking using either of these two microwave alternatives will greatly simply current steelmaking. Figure 2.3 compares the flowsheets of the MW assisted direct steelmaking with the flowsheet of traditional steelmaking.

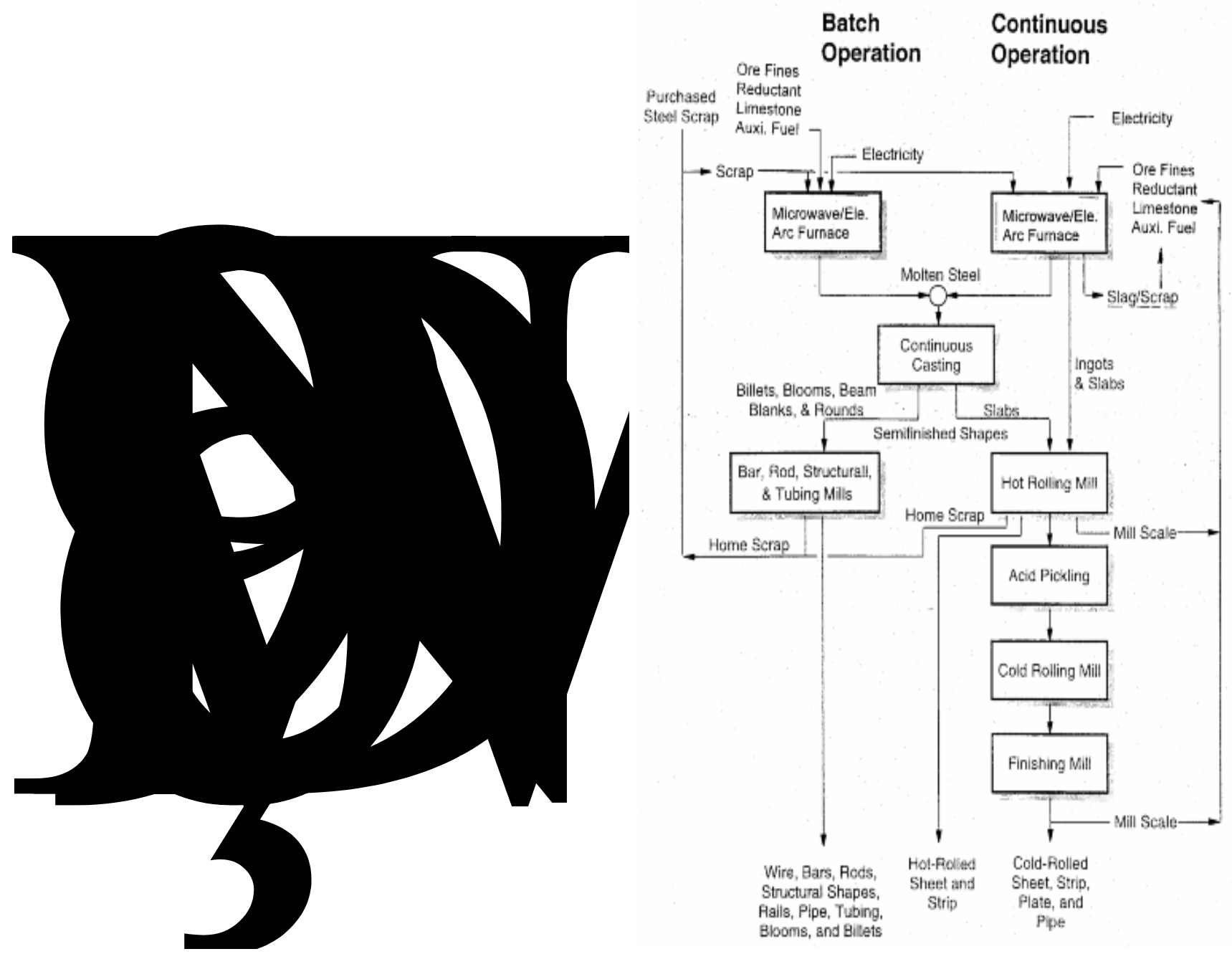

Figure 2.3. Flowsheet comparison of conventional versus MW/EAF steelmaking

From the comparison we can see that many intermediate steps would be eliminated if MW assisted steelmaking technology is adopted. Downstream hot and cold processes remain intact.

\section{3) MW/EAF Integrated Units}

The alternatives described above are integrated MW/EAF units. The integrated units simplify the operation in that there is no hot metal transfer and associated problems. However, an integrated 
unit may lower the maximum use of valuable capacity. For example, MW and EAF heating capacities are very valuable and should be used to their extremes. In integrated units, the EAF power is idle during MW reduction and MW power may be partially idle during EAF heating in the first alternative. To effectively use both MW and EAF heating, MW and EAF heating could be separated. In such arrangement, MW heating will focus on iron ore reduction and EAF heating will focus on smelting and refining.

\section{4) MW/EAF Separated Units}

There could be many sub-alternatives of separated MW/EAF units dependent on how to design the two units and link them together. One common feature of the sub-alternatives we define herewith is the transfer of hot DRI directly into an EAF. A simple case is to design a separate MW unit for reduction and use an existing EAF for smelting with a hot DRI transfer mechanism. Such MW/EAF separated units will create fewer disturbances to current EAF operation. Effort can be focused on the new MW assisted iron ore reduction unit. The unit could precede an EAF operation, forming a line of iron ore reduction, hot metal charge and smelting. Transfer of hot DRI and capacity matching between DRI and EAF need to be carefully designed for this type of setup. Figure 2.4 presents a flowsheet of steelmaking based on the use of MW/EAF separated units.

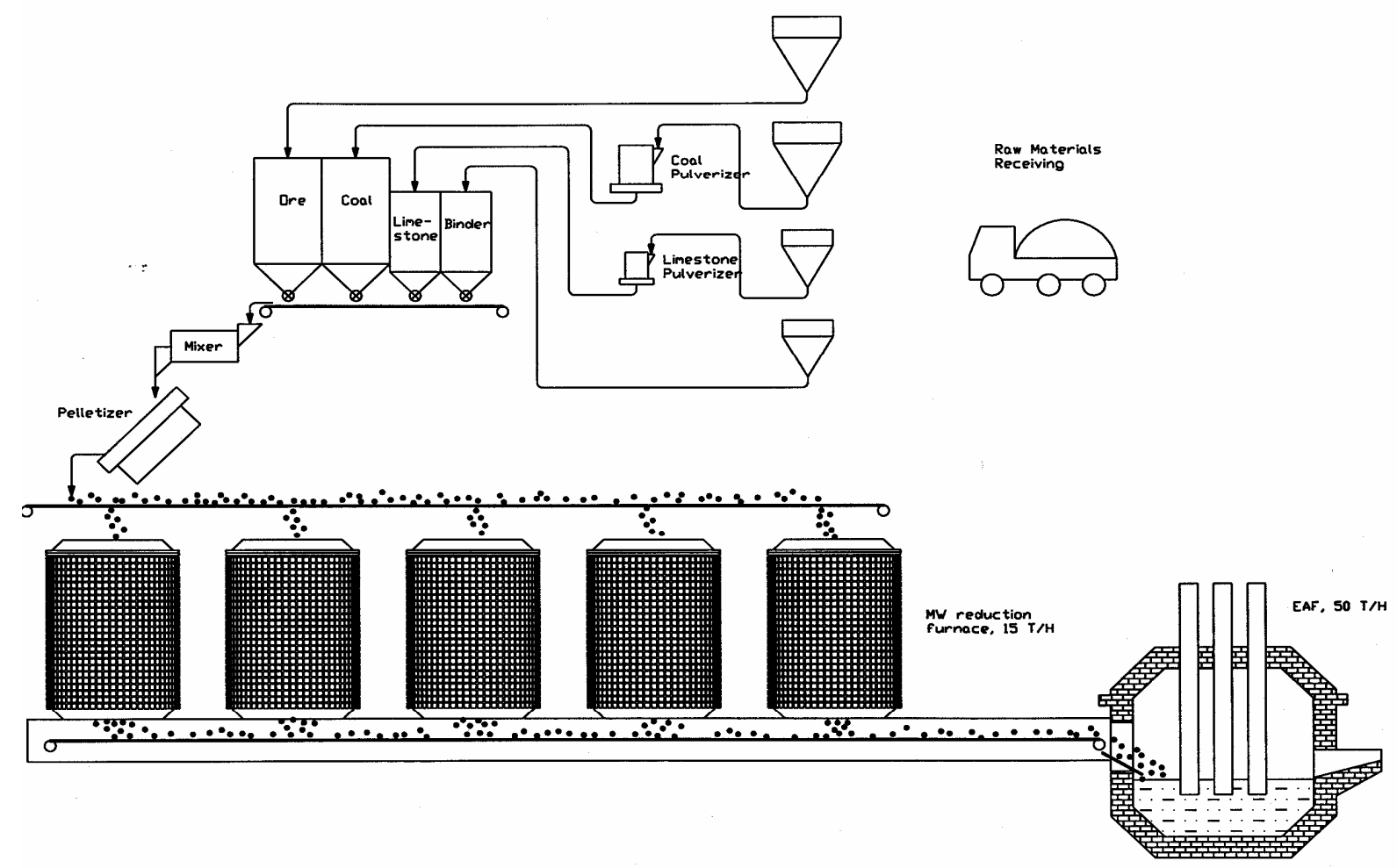

Figure 2.4. Flowsheet for steelmaking using separated MW/EAF units

A totally new, separated MW/EAF unit could be designed as well. Noramco, a steelmaking engineering firm, constructed a conceptual design of how to build a MW/EAF unit based on multiple $1.5 \mathrm{~kW}$ MW generators. Figure 2.5 presents the conceptual design. In the design, fourteen iron oxide reducing hoppers are set above a submerged are furnace (SAF). Each 
reducing hopper has multiple $1.5 \mathrm{~kW}$ magnetrons mounted on its wall. Mixed raw material is fed into each hopper through an annular slipstick conveyer. Iron oxide is reduced by MW irradiation and discharged into the underneath SAF. Molten steel and slag are continuously produced.

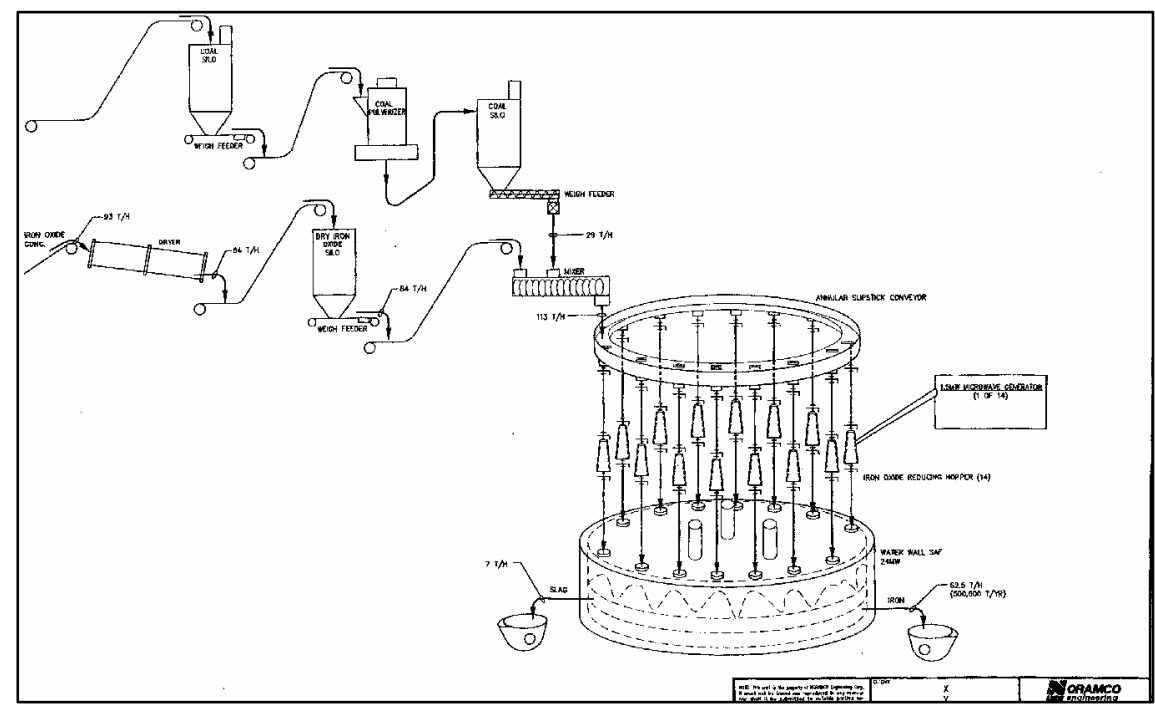

Figure 2.5. Conceptual design of MW/EAF unit based on $1 \mathrm{~kW}$ MW generator.

The MW unit could be totally separated from the EAF and operated as an independent entity, producing cold DRI as its product. This MW entity could sell DRI to mini mills. That method of operation may lower overall energy efficiency and cause reoxidation due to hot DRI cooling. However, it eliminates the problem of matching two operations.

\section{5) MW Assisted DRI Production (Hybrid Heating)}

As an alternative to direct steelmaking in an integrated MW/EAF unit as discussed above, there is the possibility of utilizing MW heating to assist DRI production. In this alternative, DRI is produced by combining conventional heating with MW heating. The purpose is to significantly shorten the reduction time and lower the reduction temperature of currently used DRI processes.

The current DRI processes can be classified into four categories: Gas/Shaft, Gas/Fluid Bed, Coal/RHF, and Coal/Fluid Bed. The common problem in conventional DRI processes is low reduction efficiency due to 1 ) slow heat transfer from outside to inside through the powdered, poorly thermal conducting material, and 2) slow mass transfer of reducing gas and product gas between outside and inside through the porous iron ore pellets. Possible use of MW heating in these processes to improve their performance is discussed in the following sections. 


\section{Gas/Shaft Process}

Figure 2.6 illustrates an example of the gas/shaft process. This process blows hot $\mathrm{CO}$ and $\mathrm{H}_{2}$ through iron ore contained in a shaft furnace to reduce the ore. The hot mixture of $\mathrm{CO}$ and $\mathrm{H}_{2}$ is the only heating source to heat the ore. $\mathrm{CO}$ and $\mathrm{H}_{2}$ also function as the reducing gases. To

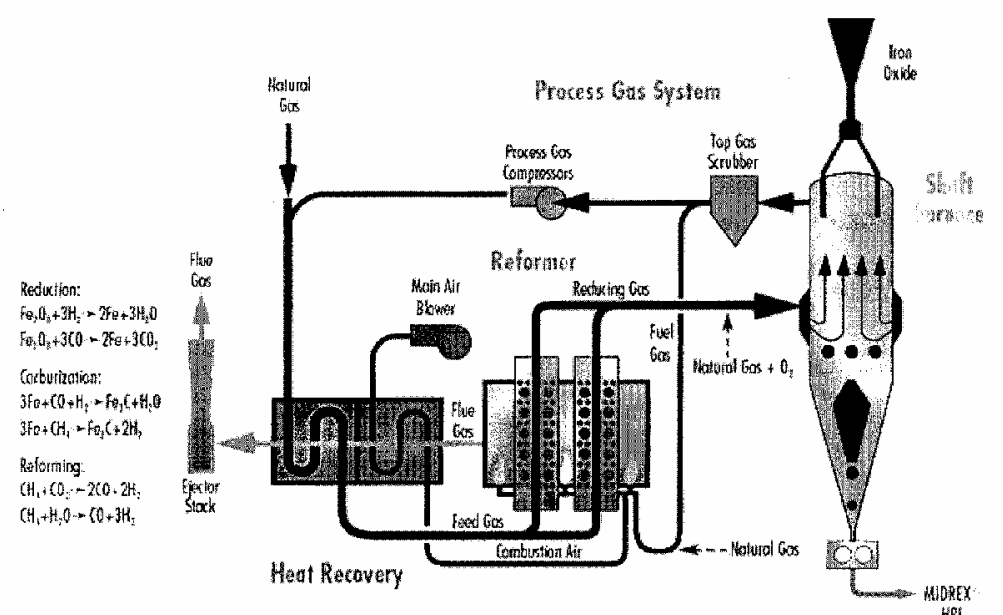

Figure 2.6. Gas/shaft DRI process generate the mixture of $\mathrm{CO}$ and $\mathrm{H}_{2}$, natural gas is utilized. The natural gas is mixed with $\mathrm{CO}_{2}$ and/or $\mathrm{H}_{2} \mathrm{O}$ and heated by a fuel in a reformer to form $\mathrm{CO}$ and $\mathrm{H}_{2}$. The gas is of low heat capacity. It passes heat to iron ore primarily through convection heating. It is not practical to get $\mathrm{CO}$ and $\mathrm{H}_{2}$ to very high temperature for increasing heat transfer. The gas operation temperature is about $900^{\circ} \mathrm{C}$. There are also many heat losses in the entire system. The energy efficiency is low.

MW heating can be utilized in this process to eliminate the reformer and change the heating source from hot gases to iron ore volumetric heating. Figure 2.7 illustrates the potential change. In this alternative, MW will heat iron ore in the shaft to the desired reducing temperature in a very short time. Natural gas will be circulated through the shaft in conjunction with exhaust gases, which are the source of $\mathrm{CO}_{2}$ and $\mathrm{H}_{2} \mathrm{O}$. The hot bath of iron ore is an in-situ reformer to convert hot natural gas into hot $\mathrm{CO}$ and $\mathrm{H}_{2}$ which will be the reducing agents. This modified DRI process has the potential of higher energy efficiency and higher production rate.

\section{$\underline{\text { Rotary Kiln }}$}

Figure 2.8 presents a flow diagram of this type of DRI processes. This process uses coal as the reducing agent. Iron ore blended with limestone and coal is fed into a rotary kiln. Natural gas or oil is used as fuel to produce flames to heat the materials and the wall of kiln. Heat is transferred to the materials through radiation, convection and direct contact with the

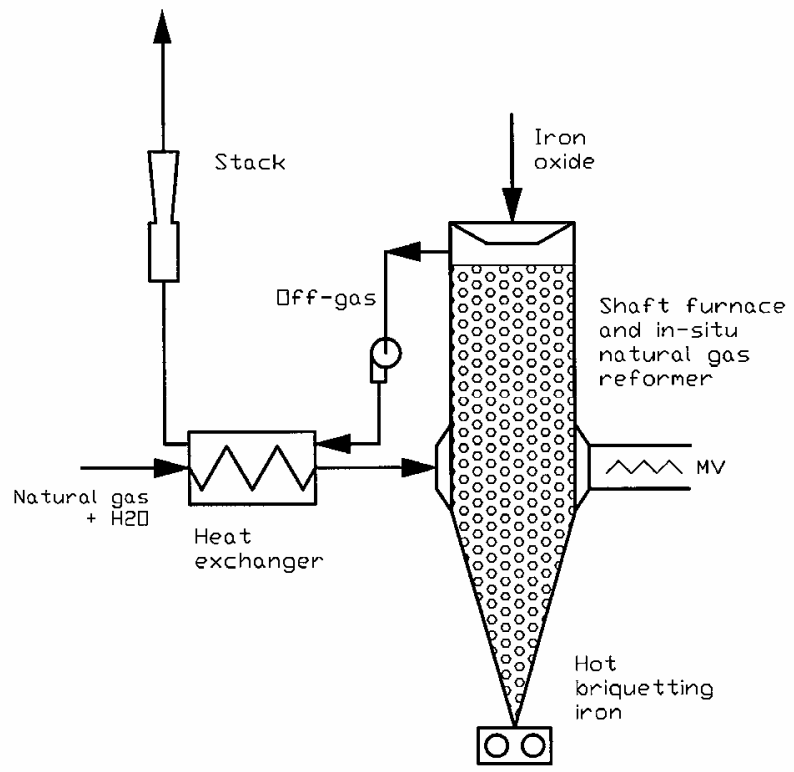

Figure 2.7. MW assisted gas/shaft process rotating wall. A large amount of heat is lost, carried away by exhaust gases, because of large free space above the materials in the kiln. The energy efficiency is low in this kind of furnace. 


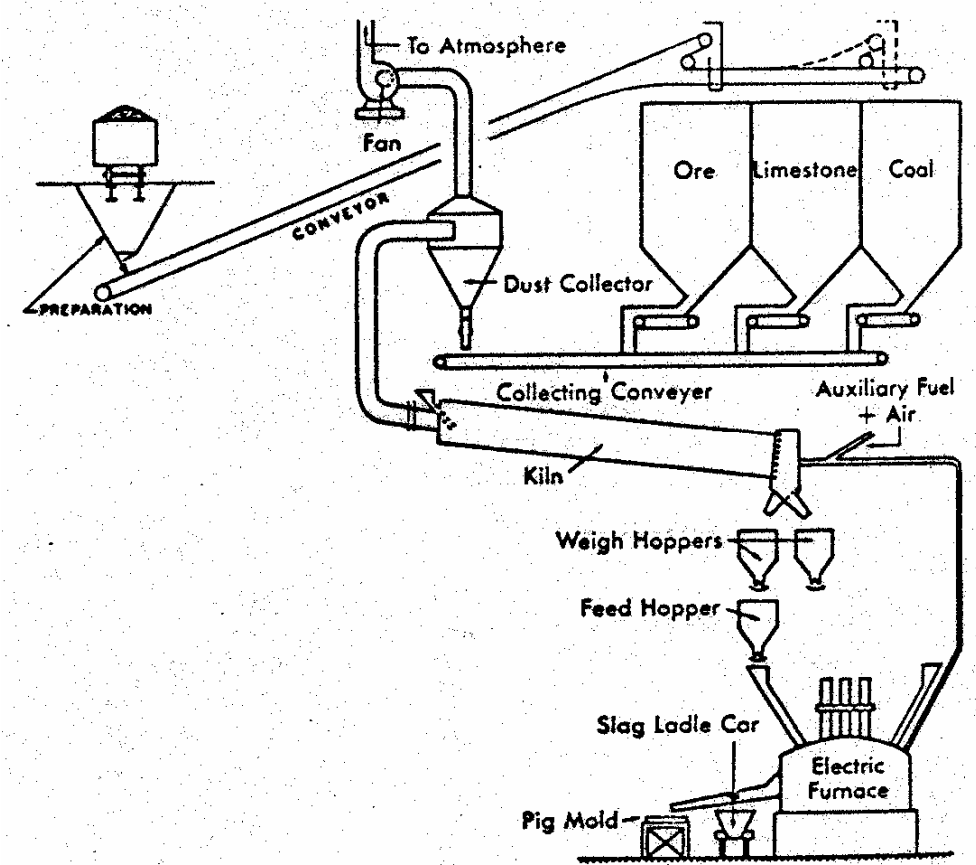

Figure 2.8. Rotary kiln DRI process
It is possible to replace fuel combustion with MW heating in a rotary kiln DRI process. The MW heating zone will be located near the exit port of the kiln. The raw material in that region will be heated to an elevated temperature in a short period of time. The coal in the raw materials will react initially with $\mathrm{O}_{2}$ in air to form CO. In the reducing atmosphere at the elevated temperature under MW catalytic irradiation, iron ore will be quickly reduced. The exhaust gases consisting of volatiles from coal, $\mathrm{CO} / \mathrm{CO}_{2}, \mathrm{H}_{2} \mathrm{O}$ and other gases will pass through the rest of the materials in the kiln to transfer heat from the gases to the materials. Because the exhaust gases at the exit port of kiln still contain fairly high amounts of hydrocarbons, $\mathrm{CO}$ and $\mathrm{H}_{2}$, the gases will be circulated back to the kiln as a gaseous reducing agent. The hydrocarbons in the coal volatiles will be converted into $\mathrm{CO}$ and $\mathrm{H}_{2}$ in the MW heating region with the presence of $\mathrm{CO}_{2}$ and $\mathrm{H}_{2} \mathrm{O}$. If necessary, additional air and water will be injected into the stream to facilitate the formation of $\mathrm{CO}$ and $\mathrm{H}_{2}$. Because there won't be air required for combustion, the raw materials can be more fully charged into the kiln. There will be much less exhaust gases released to air. Therefore the energy efficiency is expected to be much higher. There is potentially no need for a dust collector. The dust will be circulated back into the kiln along with the exhaust gases. Figure 2.9 illustrates the concept.

\section{Fluidbed DRI Process}

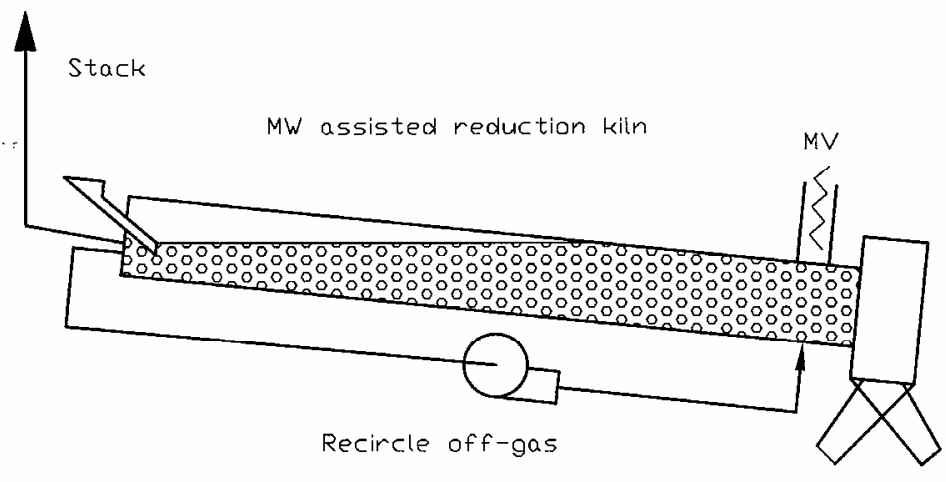

Figure 2.9. MW assisted rotary kiln DRI process

Figure 2.10 is the flow diagram of a fluid bed DRI process. This process uses nearly pure hydrogen as the reductant. Iron ore in the size of -10 mesh +325 mesh is preheated to $900-$ $1000^{\circ} \mathrm{F}$ in a rotary kiln or a fluid bed heater. The hot ore is then transferred to a charge hopper which is pressurized to 600-650 psi. The ore is transferred continuously from the charge hopper to the topmost bed of the reactor. A portion of the total reduction is done in the top bed by partially spent hydrogen. The solids are then dropped to the next lower bed which has been emptied by dropping its solids to yet a lower bed. Further reduction continues in the second bed and the solids are transferred in like fashion to the next lower bed. The beds from bottom to top 


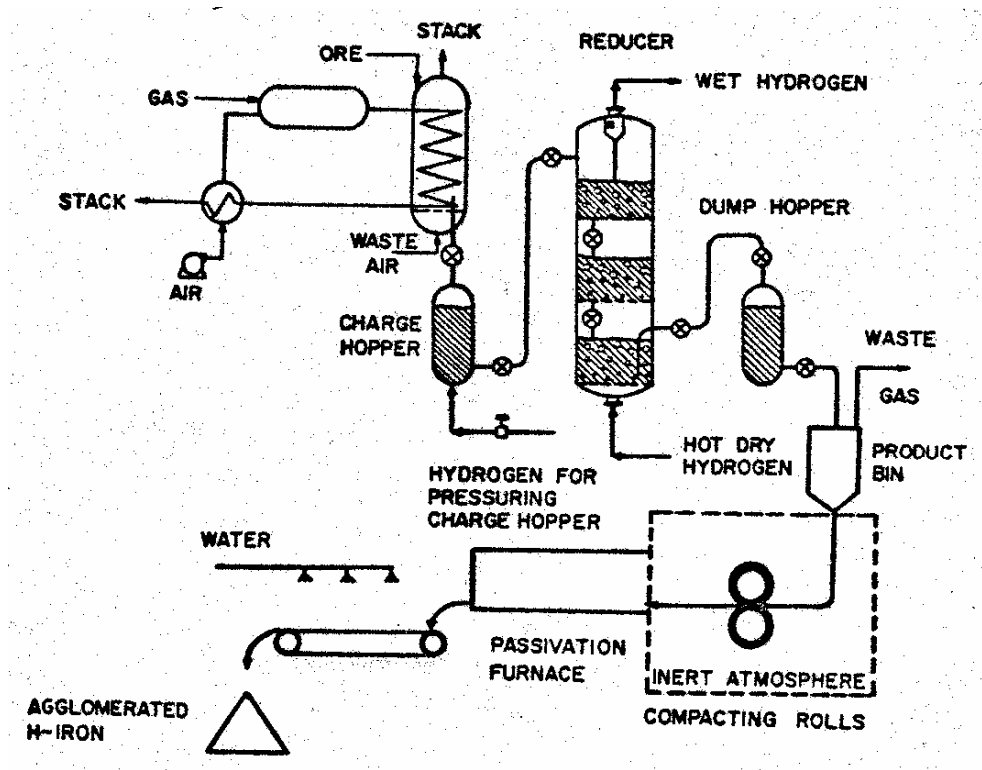

Fig. 9. Flow diagram of ore processing for the H-Iron process. Source is reference 40 .

Figure 2.10. Fluidbed DRI process are treated successively with reducing gas lower in hydrogen content, the bottommost bed receiving the gas of highest hydrogen content and therefore of greatest reducing potential. Reduced fines, pyrophoric in nature, are transferred from the bottom bed to a pressurized dump hopper. The dump hopper is purged of hydrogen and pressurized to 150 psi with a nonoxidizing gas to permit transporting of the powder to subsequent processing steps of passivation and compacting. Charging and dumping are carried out without depressurizing the reducer or interrupting the flow of hydrogen. The reduced fines have essentially the same size consist as that of the feed.

In this process, the iron ore and hydrogen have to be preheated in separate facilities using a fuel. If MW heating is utilized, we believe it could dramatically simplify the system and increase its energy efficiency. MW irradiation can be easily introduced into the fluid bed reducer by waveguides. Iron ore fines will be heated and held at a desired reducing temperature by MW heating. The exhaust gas will be circulated back into the reducer for auxiliary heating and reduction. The atmosphere is controlled by continuously charging fresh reducing gas into the reducer. Figure 2.11 illustrates the new flow diagram of the MW heated fluid bed DRI process.

\section{$\underline{\text { Traveling Grate Process }}$}

Traveling grates are widely used in the combustion of coal, charring of carbonaceous matter, metallic ore sintering, thermal extraction of sulfur, pelletizing of ore concentrates, production of Portland cement clinker, calcination of limestone, and production of
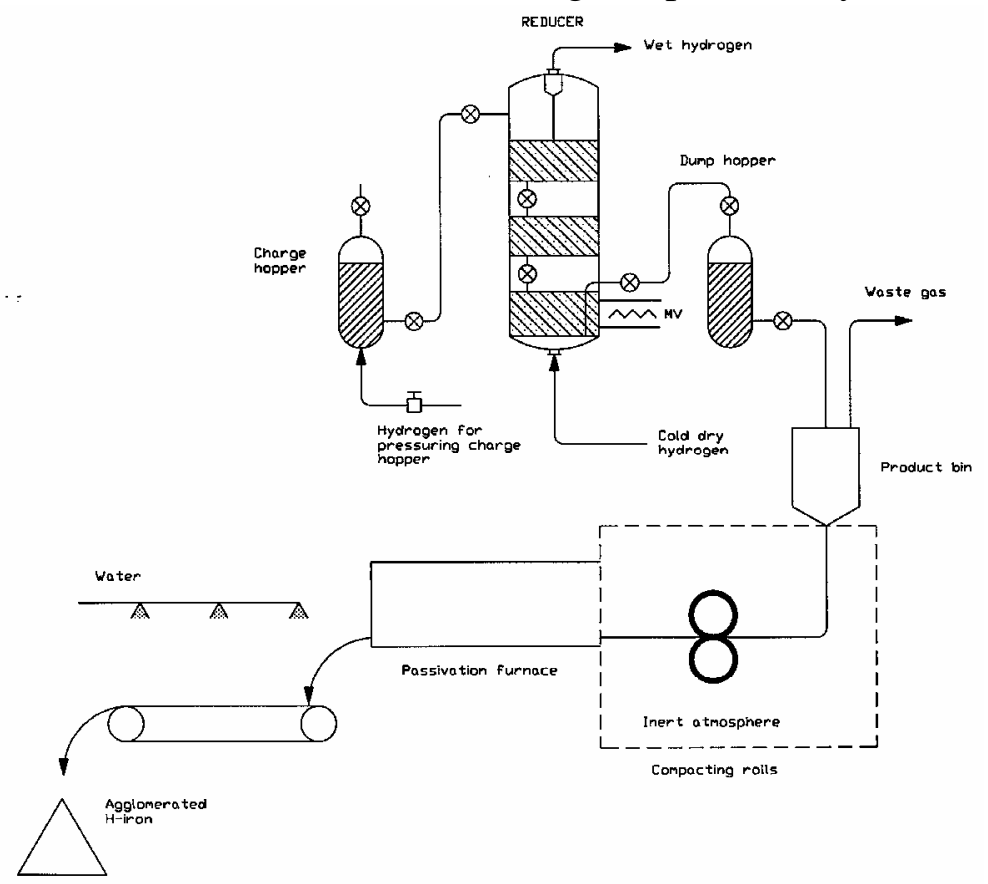

Figure 2.11. MW assisted fluidbed DRI process
Institute of Materials Processing Michigan Technological University March 31, 2005 
lightweight aggregate. These traveling grates can be divided into three types: chain, circular and Dwight-Lloyd. Dwight-Lloyd and circular traveling grates have been used for DRI production. Figure 2.12 illustrates the flow-sheet of Dwight-Lloyd traveling grate application. In this application, the feed material consisting of iron ore, returned fines, limestone and coal is mixed, pelletized using a flying saucer, and charged into the traveling grate, where the feed material is heated by burners. The coal in the feed material is the reducing agent and assures a reducing atmosphere in the chamber. The coal is over dosed in order to reduce iron with higher carbon content, even to form iron carbide. To achieve quick heating and reduction, the feed material is thinly layered on the grate and the grate chamber temperature is as high as $2500^{\circ} \mathrm{F}$. The feed material is heated primarily through radiation and convection. Similar to the rotary kiln and shaft processes, the energy efficiency is low. The carbonized pellets are then fed into a submerged arc

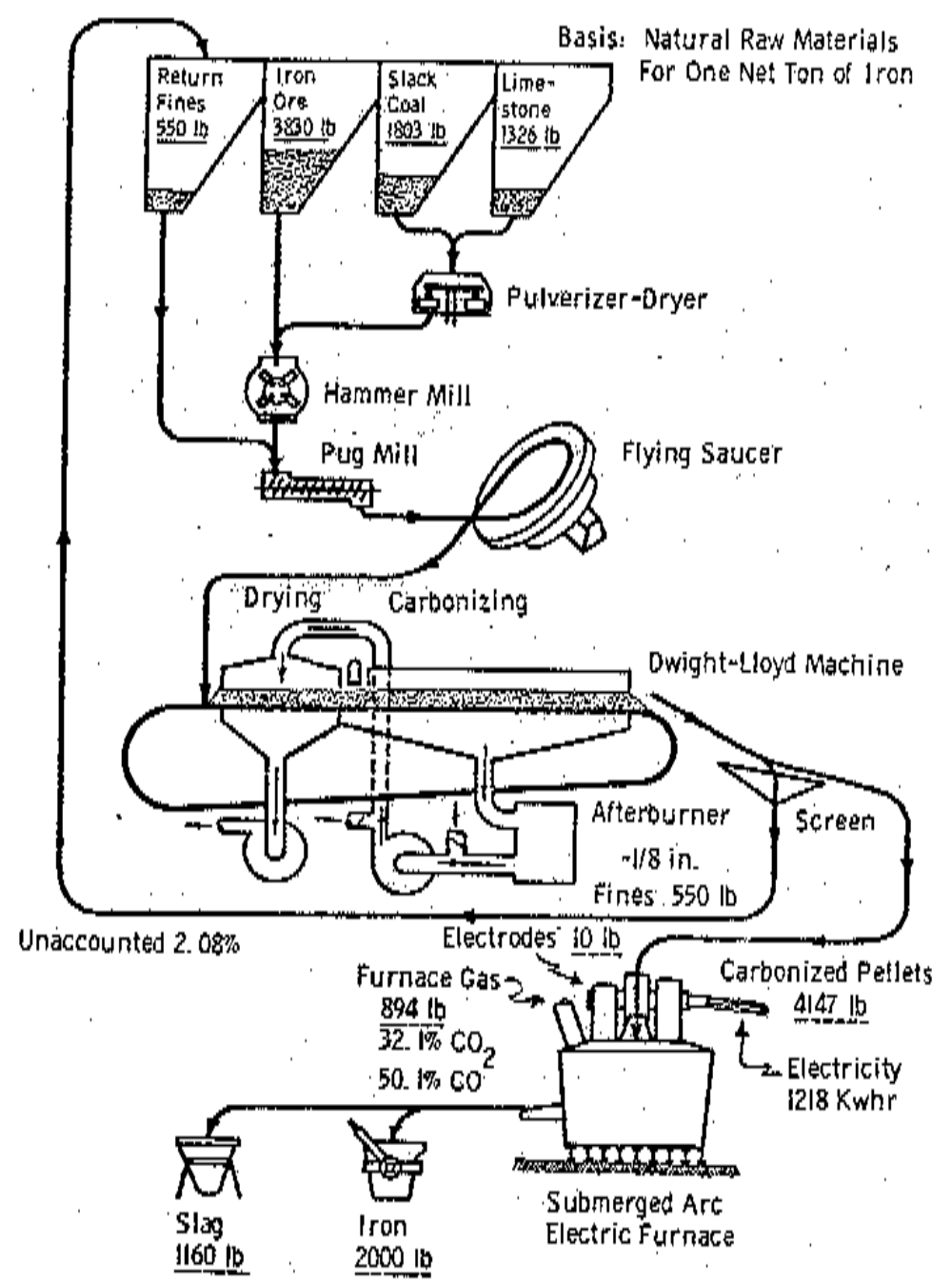
electric furnace to produce molten slag and iron.

The traveling grate DRI processes could be modified by replacing the burners with MW heating. In this application, MW will be introduced into a traveling grate reduction chamber, or a DwightLloyd Machine near the discharge port of the grate as shown in Figure 2.13. The feed material

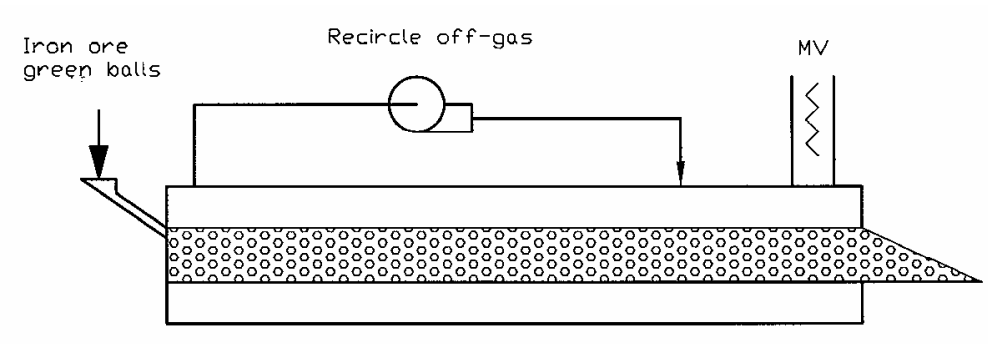

Figure 2.13. MW assisted traveling grate DRI process may not change but could be layered on the grate much thicker in comparison with a conventional traveling grate DRI process. The coal in the feed material still will be the reducing agent. The exhaust gas containing hydrocarbon, $\mathrm{CO}$, $\mathrm{CO}_{2}, \mathrm{H}_{2} \mathrm{O}$ and heat will be circulated back in near the 
discharge port of the grate to utilize the thermal and chemical energies of the gas. Again, due to the replacement of external heating by burners with internal heating by MW irradiation, it is expected to greatly improve energy efficiency and production rate as well as lower the reduction temperature and increase traveling grate life.

\section{$\underline{\text { Rotary Hearth DRI Production }}$}

This process is similar to a circular traveling grate process. Instead of using a circular traveling grate, this process uses a rotary hearth to carry out the reduction as shown in Figure 2.14. MW irradiation could be introduced into the rotary hearth as auxiliary heating with the potential benefits of thicker layer of iron ore, shorter reduction time, lower reduction temperature, higher production rate, greater energy efficiency, and lower production cost.

\section{6) MW only DRI Production}

Our experiments have shown that an enclosed MW reactor has much higher energy efficiency and higher productivity than an open MW reactor when used to reduce iron ore. Our experiments and theoretical analysis on energy efficiency indicate that the exhaust

\section{Midrex/Kobe Steel Rotary Hearth Technology}

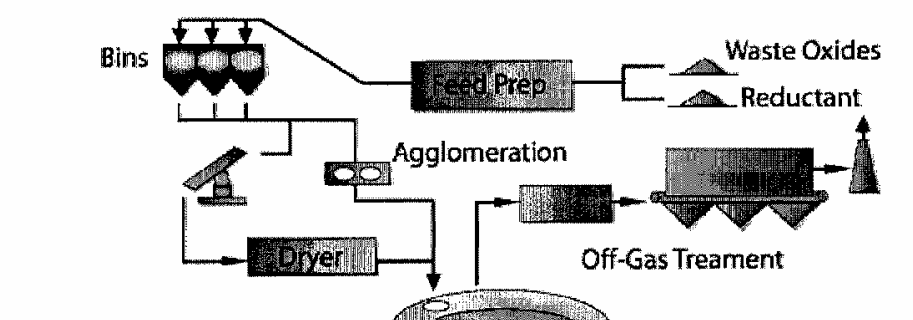

FASTMET

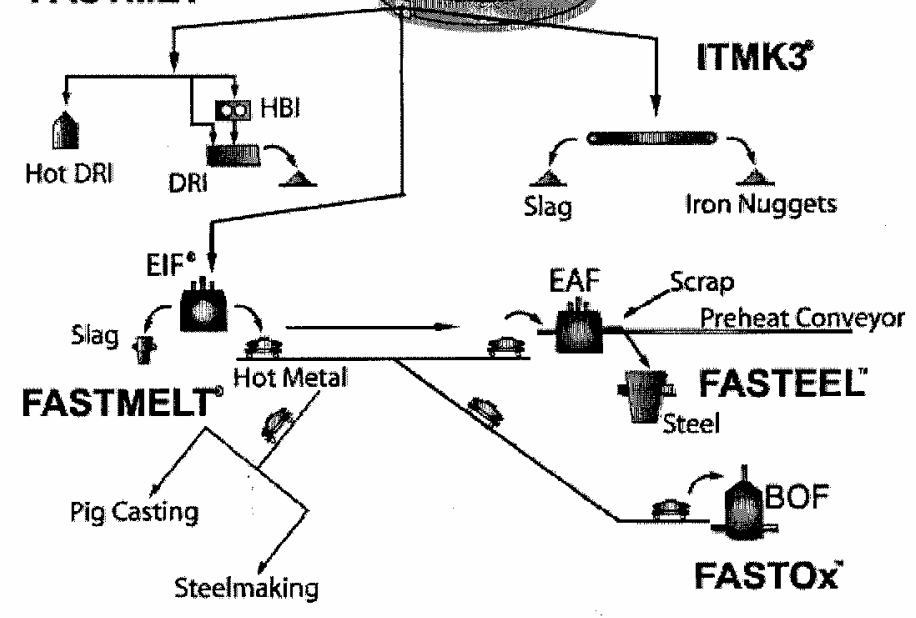

Figure 2.14. Rotary hearth DRI production gas contains a great amount of energy in the form of hot and combustible gases, which should be utilized. However, if the concentrations of the combustible gases are not controllable or high enough, it will be difficult to effectively utilize these gases, which is the situation many combustion facilities are facing. A MW reactor does not depend on air or oxygen flow for heating. It is possible to control the generation or the concentration of combustible gases in the exhaust stream of an enclosed MW reactor. To take advantage of an enclosed MW reactor and meet the need for high efficiency DRI production, a MW-only, enclosed DRI production unit could be developed. In this alternative, iron ore is mixed with pulverized coal in an amount over what is needed for reduction. At the beginning of MW heating, highly combustible volatile matter will come out of the coal and exhaust as a fuel through a pipe in the enclosed reactor. As the temperature goes up and all volatiles exhaust, $\mathrm{O}_{2}, \mathrm{CO}_{2}$ or $\mathrm{H}_{2} \mathrm{O}$ could be injected into the chamber to react with the hot coal and generate $\mathrm{CO}$ or $\mathrm{H}_{2}$ during iron ore reduction. The MW enclosed reactor functions as a coal gasifier or natural gas reformer to some extent. The targets 


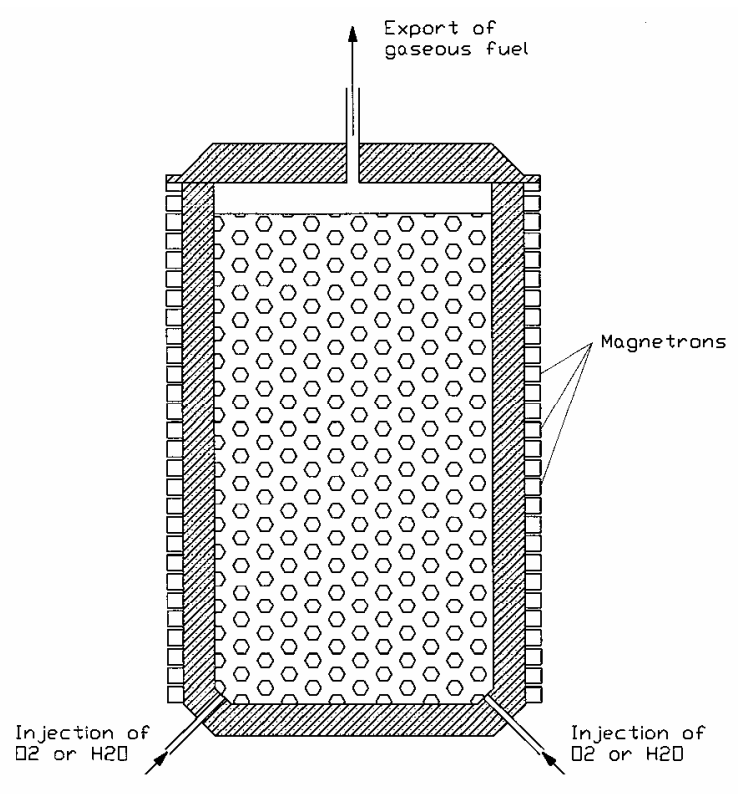

Figure 2.15. MW DRI reducing furnace and gaseous fuel generator of this alternative are to 1) reduce iron ore at high energy efficiency and high productivity, and 2) generate and export high quality gaseous fuel. Figure 2.15 shows a schematic of this alternative.

We believe there are many good opportunities for microwave technology to be utilized in the steelmaking industry. The use of microwaves can help the industry advance its production technology to a new level. 


\section{Task 3. Evaluation of Equipment, Supplies, and Worker Environment}

\section{Equipment and Supplies}

Information is provided for microwave and radio frequency equipment, along with data for electric arc furnaces.

\section{Microwave Equipment}

Microwave heating was discovered in 1950 and led to microwave ovens for home use in 1960. Today, microwave ovens are very popular and form a huge market after several decades of development. However, industrial microwave heating has a long way to go. At present, Cober Electronics, Microdry Inc., and Astex/Gerling Laboratories, Inc are the three major suppliers of industrial microwave heating equipment in the United States. Industrial microwave heating equipment includes more components than home microwave ovens, such as various sizes of generators, waveguides, circulator, monitoring instrumentation, applicators, and conveyors. Figure 3.1 illustrates an industrial MW heating system.

Microwaves are generated from magnetrons. Currently, the largest single magnetron produced is $100 \mathrm{~kW}$. However, large microwave systems do not necessarily have to have large magnetrons.

Existing Microwave Heating, Hybrid

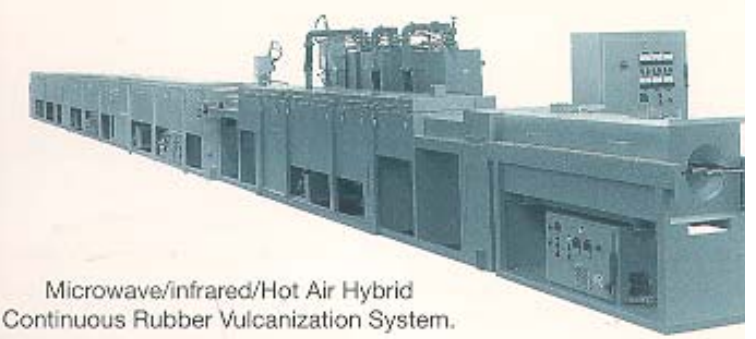

Figure 3.1. Industrial Microwave Heating System Magnetrons can be connected in parallel to multiply the power output. Large microwave facilities such as 1000KW have been employed in many industries. In 1997, EMR Microwave Technology Corporation, a Canadian company, built a pilot plant microwave metallurgy system

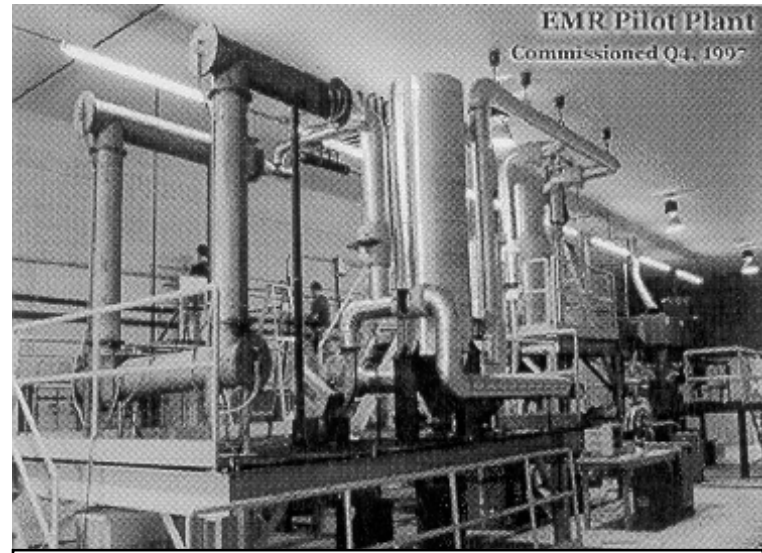

Figure 3.2. Microwave system to calcine refractory gold ores and concentrates to calcine refractory gold ores and concentrates. The system, which can process 4-20 tons of concentrate per day, is shown in Figure 3.2. A larger plant capable of processing 1000 tons of concentrate per day is in the engineering design stage. Also, $100 \mathrm{MW}$ and $10 \mathrm{GW}$ high energy microwave systems are under development.

Microwave oven cost has decreased dramatically in recent years due to high volume production and technical progress. A $1 \mathrm{~kW}$ microwave oven for home use sells for about $\$ 50$ while a $1 \mathrm{~kW}$ industrial microwave device costs about $\$ 1,000$. The major progress in industrial microwave equipment is beginning in production of high power microwave generators. A single $100 \mathrm{~kW}$ microwave generator is on market. However, 
there is no expectation to lower the cost of industrial MW generators to the level of home use microwave ovens in the near future.

One strategy to deal with the situation is to develop MW assisted steelmaking facilities based on the use of small MW generators. The obvious advantage is low equipment cost. An $1 \mathrm{~kW} \mathrm{MW}$ oven sells about $\$ 50$ which include a metal box, a MW leakage free door, a rotating system, an electronic controller, a fan, a MW generator, a transformer, and a capacitor. To build a MW assisted facility for steelmaking, we only need to use the MW generator, the transformer and the capacitor. Figure 3.3 shows a $1 \mathrm{~kW}$ MW generator mounted on a MW digestion system we built in our lab. These three parts may only cost about \$20. Another advantage is potentially more homogeneous distribution of MW energy in the materials to be heated. Many small MW generators will be mounted on the wall of a chamber. Microwaves are introduced into the chamber from each generator in all directions. The steelmaking raw material charge is an excellent MW absorber and placed in large quantity in the MW field. It is expected that this MW irradiation arrangement is more homogeneous than irradiation with microwaves introduced through a single waveguide. Figure 3.4 illustrates a schematic of a MW DRI furnace based on the use of multiple small MW generators. A $1 \mathrm{~kW}$ MW generator needs a space about 5" by 6". A chamber 9 feet in diameter and 24 feet tall could house $32001 \mathrm{~kW}$ generators. Theoretically, current MW equipment manufacturing technology could be used to construct a large facility for steelmaking use.
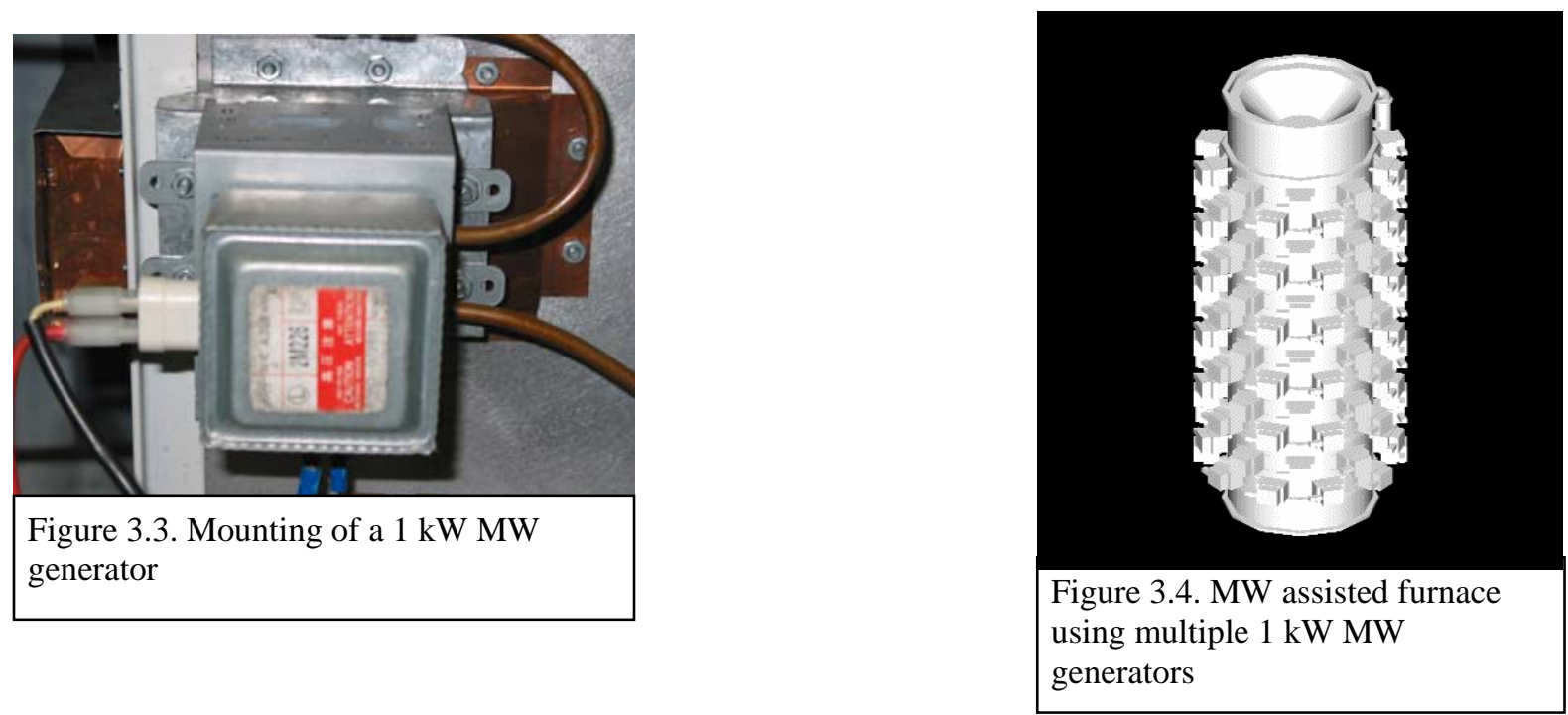

\section{Radio Frequency Equipment}

Radio frequency energy is another potential heating source to be utilized for steelmaking. An experiment done at a RF equipment manufacturer has shown that iron ore is a good absorber of RF irradiation. In comparison with microwave heating units, RF heating equipment is cheaper and higher power generators are available on the market. PSC Inc is the primary RF heating equipment manufacturer in the United States, manufacturing RF heating equipment for many industries as shown in Figure 3.5. Figures 3.6 and 3.7 present two RF units that PSC have built. PSC has the capabilities of manufacturing power ranges from kilowatts to megawatts and frequency ranging from kilohertz to megahertz. 


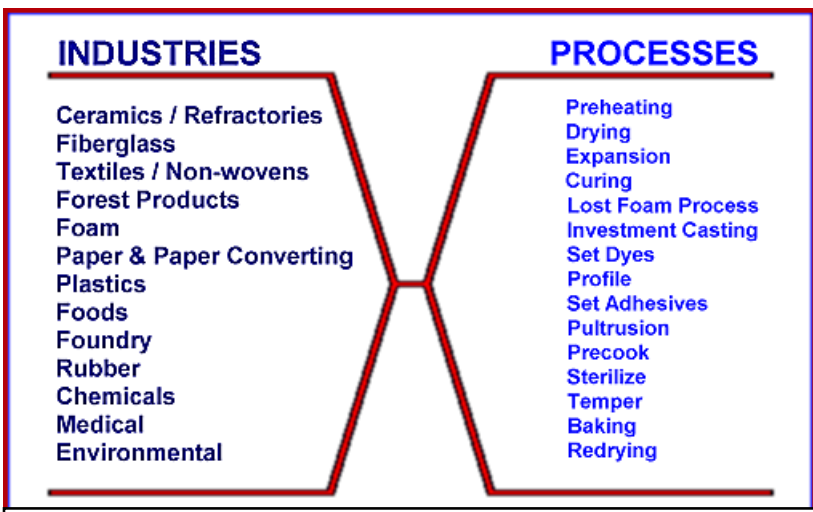

Figure 3.5. Industries and processes that utilize radio frequency heating
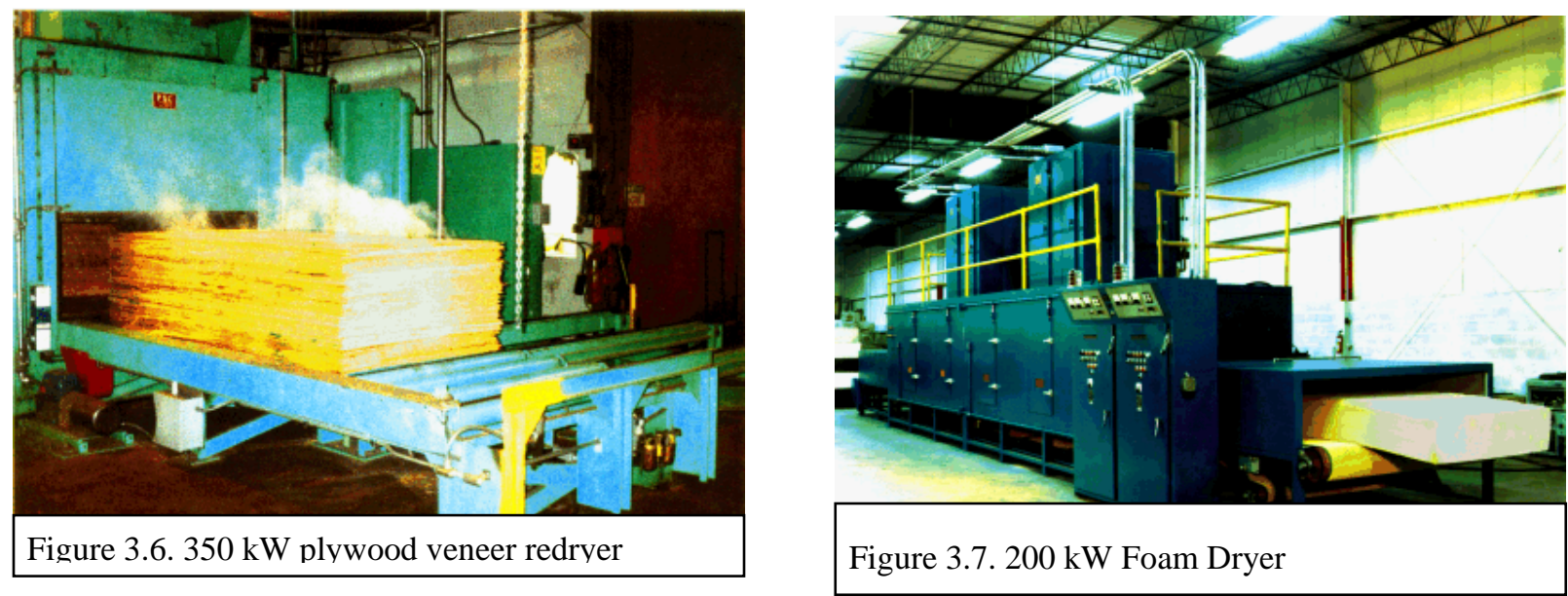

\section{$\underline{\text { EAF Manufacturers }}$}

There are two major EAF manufacturers in the United States, EMC International, Inc., a subsidiary of Midrex Enterprise, Inc., and Techint Technologies Inc. (who owns Lectromelt). This new steelmaking technology will require modification of the existing EAFs, or the design and manufacture of very different MW/EAF units. It is likely these companies will be involved in commercial equipment development.

\section{Worker Environment}

The workers in the new MW steelmaking plants will work in an environment similar to the current mini mills, with the additional concern of potential microwave leakage. Great attention should be given to worker safety by preventing microwave leaks. Online monitoring for microwave leakage, leak alarms, and emergency MW power shutdown systems should be designed and installed. 
For microwave operations, the primary health effects of concern from electromagnetic radiation are thermal exposure or burns and contact shocks, although research is continuing to investigate non-thermal effects. OSHA has voluntary standards for workplace exposure to non-ionizing radiation (including microwaves). The recommended power density exposure limit is 10 milliwatt $/ \mathrm{cm}^{2}$ for periods of 0.1 -hour or more. An energy density limit of 1 milliwatt-hour $/ \mathrm{cm}^{2}$ during any 0.1 hour period is also recommended. These standards have been ruled unenforceable. Some U.S. states with their own OSHA-type programs, however, are enforcing this or other exposure limits. The OSHA standard also specifies the design of a warning sign, although the inclusion and choice of warning information or precautionary instructions is discretionary. More information regarding these regulations is given in Task 7. 


\section{Task 4. Evaluation of Steel Company and Supplier Company Interaction and Logistics}

Microwave assisted steelmaking seems very different from the traditional steelmaking and may lead to dramatic changes to the current steelmaking chain. The steelmaking chain consists of raw material supplies, logistics and steelmaking. Prediction of the technology impact on the current steelmaking chain will help to plan a technology implementation strategy.

\section{Current interactions and logistics}

Figure 4.1 shows the locations of steel plants in North America in the 1990's. This figure was prepared by American Iron and Steel Institute. Many of the plants shown did not survive the downturn of the U.S. steel industry during 1998-2002. They either declared bankruptcy or were acquired by other steelmakers, mostly by foreign steelmakers from Canada, Mexico, German, Brazil, Russia and China. The bankrupt companies included some large and well-known companies such as Bethlehem Steel Corporation, Geneva Steel, LTV Steel Company, National Steel Corporation, North Star Steel Company, Rouge Steel Company, and Weirton Steel Corporation.

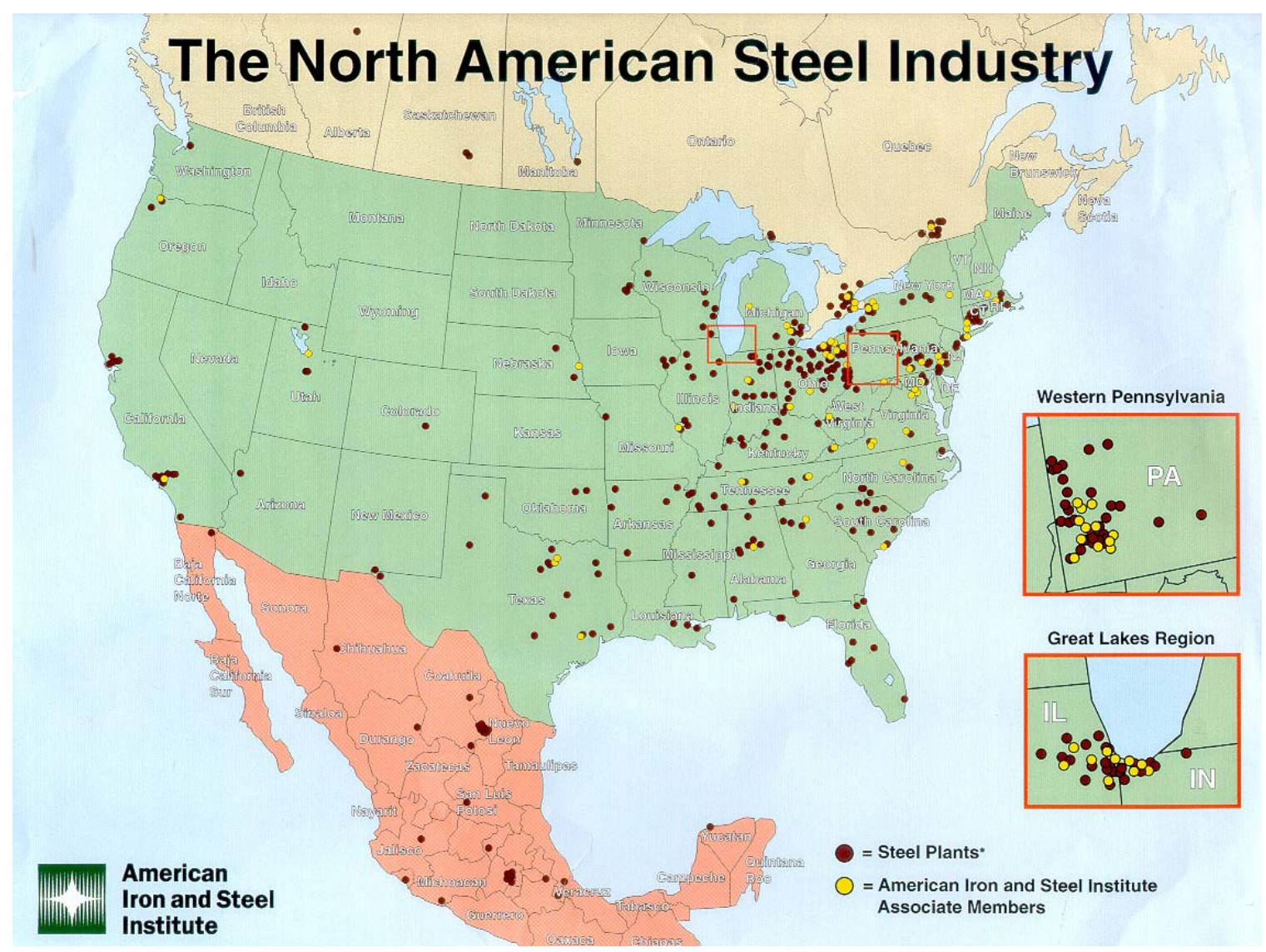

Figure 4.1. The North American steel industry.

Figure 4.1 includes facilities that do not make steel, such as cold rolling mills, pipe extrusion and electrolytic coating operations. MW assisted steelmaking may not have much effect on these 
downstream operations. To simplify the evaluation, only current steelmaking companies in the United States or the companies having a close supply relationship with steelmakers were studied. Table 4.1 gives their basic information.

\begin{tabular}{|c|c|c|c|c|}
\hline \multicolumn{5}{|c|}{ Table 4.1. U.S. Steel Plants } \\
\hline COMPANY & WEB ADDRESS & OPERATION & $\begin{array}{c}\text { MAIN } \\
\text { LOCATIONS }\end{array}$ & $\begin{array}{l}\text { ANNUAL } \\
\text { SALES }\end{array}$ \\
\hline A. Finkl \& Sons Co. & www.finkl.com & EAF & IL & $25-100 \mathrm{M}$ \\
\hline AK Steel Corp & $\overline{\text { www.aksteel.com }}$ & BF/BOF, EAF & $\mathrm{OH}, \mathrm{IN}, \mathrm{KY}$ & $5.2 \mathrm{~B}$ \\
\hline Algoma Steel Inc. & www.algoma.com & BF/BOF, coking & Ont. & $1.3 \mathrm{~B}$ \\
\hline Beta Steel Corp & www.betasteelcorp.com & EAF & IN & $25-100 \mathrm{M}$ \\
\hline $\begin{array}{l}\text { Charter Manufacturing } \\
\text { Company, Inc. }\end{array}$ & www.chartermfg.com & EAF & WI & $50-250 \mathrm{M}$ \\
\hline CitiSteel USA, Inc. & www.citisteel.com & EAF & $\mathrm{DE}$ & $50-250 \mathrm{M}$ \\
\hline Cleveland-Cliffs Inc. & www.cleveland-cliffs.com & Iron ore pellets & MN, OH, Que & $494 \mathrm{M}$ \\
\hline Gerdau Ameristeel & www.ameristeel.com & EAF, scrap & $\begin{array}{l}\text { FL, GA, TN, } \\
\text { NC, } \\
\text { KY, NJ, Ont }\end{array}$ & $3 \mathrm{~B}$ \\
\hline $\begin{array}{l}\text { Gallatin Steel } \\
\text { Company }\end{array}$ & $\underline{\text { www.GallatinSteel.com }}$ & EAF & KY & $250-500 \mathrm{M}$ \\
\hline IPSCO Inc. & www.ipsco.com & EAF, scrap & Saskatchewan & $737 \mathrm{M}$ \\
\hline ISG & www.intlsteel.com & $\begin{array}{l}\text { BF/BOF, EAF, } \\
\text { coal, coking, iron } \\
\text { ore, DRI, HBI }\end{array}$ & $\begin{array}{l}\text { IN, OH, SC, IL, } \\
\text { NY, NC, MD, } \\
\text { PA, WV }\end{array}$ & $9 \mathrm{~B}$ \\
\hline Ispat Inland Inc. & www.inland.com & $\begin{array}{l}\mathrm{BF} / \mathrm{BOF} \text {, iron } \\
\text { ore }\end{array}$ & IL, IN, MN & $2.38 \mathrm{~B}$ \\
\hline $\begin{array}{l}\text { Keystone Consolidated } \\
\text { Industries, Inc. }\end{array}$ & www.keystonesteel.com & EAF & IL & $370 \mathrm{M}$ \\
\hline Rouge Steel Company & www.rougesteel.com & $\mathrm{BF} / \mathrm{BOF}$ & MI & $1.16 \mathrm{~B}$ \\
\hline Shenango Inc. & www.shencoke.com & Coking & PA & $25-100 \mathrm{M}$ \\
\hline Steel Dynamics, Inc & www.steeldynamics.com & EAF & IN & $2 \mathrm{~B}$ \\
\hline The Timken Company & www.timken.com & EAF & $\mathrm{OH}, \mathrm{PA}$ & $882 \mathrm{M}$ \\
\hline Tuscaloosa Steel Corp & $\overline{\text { www.tsteel.com }}$ & EAF & $\mathrm{AL}$ & $50-250 \mathrm{M}$ \\
\hline USX-US Steel Group & www.ussteel.com & $\begin{array}{l}\text { BF, BOF, iron } \\
\text { ore, coking }\end{array}$ & PA, AL, IN & $11.4 \mathrm{~B}$ \\
\hline $\begin{array}{l}\text { Wheeling-Pittsburgh } \\
\text { Steel Corp }\end{array}$ & www.wpsc.com & BF/BOF, coking & $\mathrm{WV}, \mathrm{OH}$ & $1.1 \mathrm{~B}$ \\
\hline
\end{tabular}

The current steelmakers can be divided into six categories:1) integrated mills operating BF/BOF and iron ore mining; 2) integrated mills operating BF/BOF; 3) mini mills operating EAF and scrap steel process; 4) mini mills operating EAF; 5) coke producer; and 6) iron ore mining and processing. The first category includes large organizations such as USX-US Steel Group and ISG. They operate not only BF/BOF facilities but also coking, iron ore mining and logistics directly. The second category includes entities that are relatively smaller in size, such as Ispat Inland and Rouge Steel. They may own some shares in iron ore suppliers, but generally speaking, they do not operate iron ore mines directly. Instead, they purchase iron ore pellets based on contracts with iron ore suppliers. The third category includes larger mini mills such as Gerdau Ameristeel and IPSCO. They have their own scrap steel collection, processing and transportation system. The fourth category includes smaller mini mills such as Beta Steel, CitiSteel, Gallatin Steel and Tuscaloosa Steel. They primarily rely on scrap suppliers to get their raw material. The fifth category is the independent coke producer, such as Shenango Inc. This company supplies coke to integrated mills. The sixth category includes iron ore processors such 
as Cleveland-Cliffs, Inc. This company operates iron ore mining and pelletizing facilities, and supplies iron ore pellets to integrated mills.

Now let's see more details of selected companies. Cleveland-Cliffs is a very typical iron ore supplier. CCI is the largest producer of iron ore pellets in North America. Cliffs sells the majority of its pellets to integrated mills in the United States and Canada. The Company operates six iron ore mines located in Michigan, Minnesota and Eastern Canada as shown in Figure 4.2. CCI-operated mines currently have the capacity to produce 37.7 million tons of iron ore pellets annually; Cliffs share represents approximately 28 percent of the total North American annual pellet capacity. The Company sells its share of iron ore production to integrated mills, generally pursuant to term supply agreements with various price adjustment provisions.

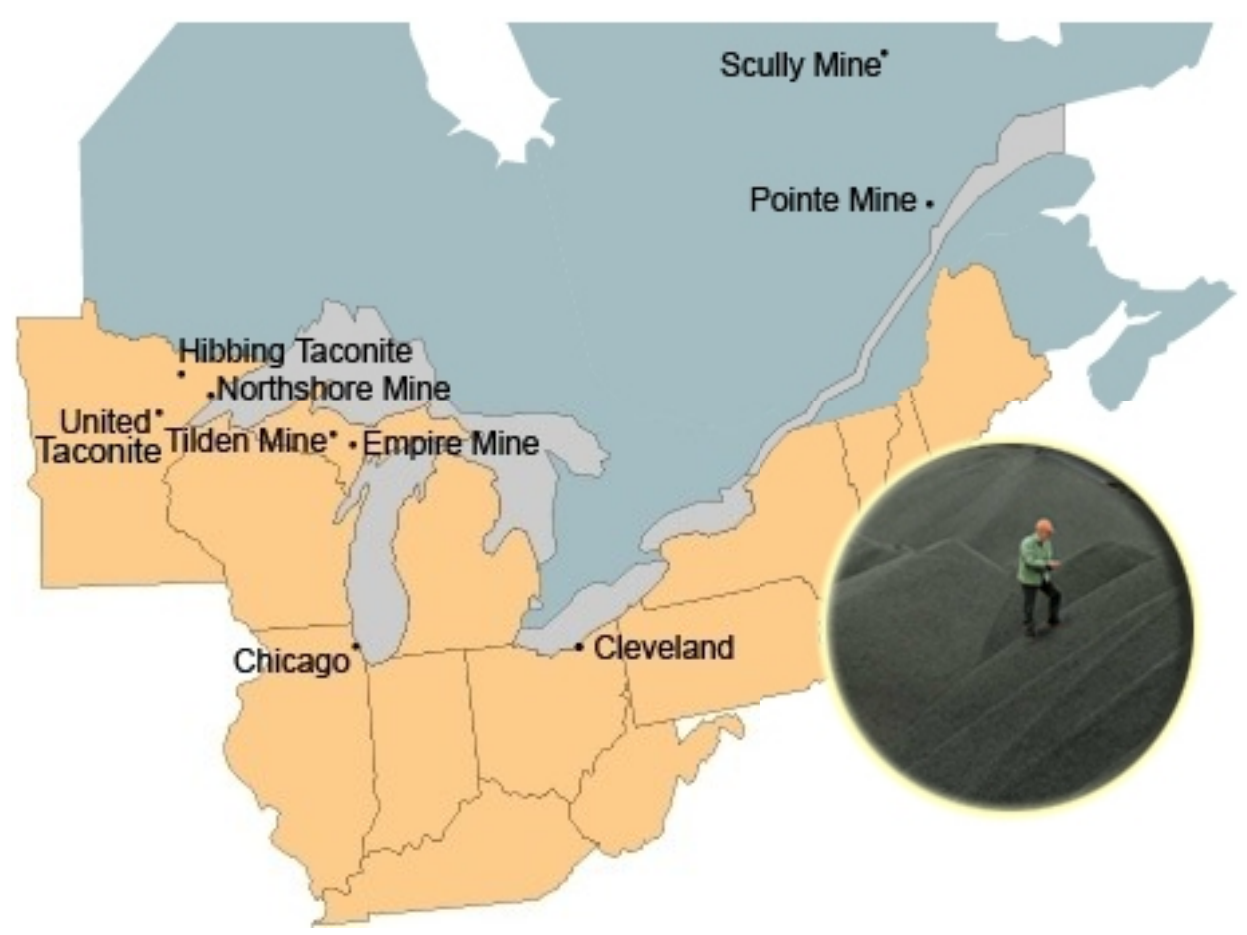

Figure 4.2. CCI mine locations

The USX-US Steel Group is the largest U.S. integrated steelmaker. U.S. Steel itself supplies iron ore and coke. Iron ore is mined and processed by its Minnesota Ore Operations at Minntac in Mountain Iron and Keewatin Taconite in Keewatin, both on Minnesota's Mesabi iron range. After being extracted from the ground, the iron ore is crushed into a fine powder and separated from impurities with the use of magnets. The concentrated powder is then agglomerated into pellets and sintered. Coke is produced at the Gary Coke Plant in Gary, IN, Clairton Works 
outside Pittsburgh, PA, and Granite City Coke Plant near St. Louis, MO. U.S. Steel also sells iron ore pellets and coke to other steelmakers.

U.S. Steel had its own Logistics Services Department to provide the logistics services needed by U.S. Steel and other steelmakers. The U.S. Steel logistics services include transportation management, operations management, contract management, administrative services, packaging and loading services, and international services. In 2004, U.S. Steel sold its raw materials and transportation businesses including its coke operations at Clairton, PA and Gary, ID, its iron ore operations at Minnesota (Minntac), and its wholly owned transportation services subsidiary Transtar, Inc. (Transtar), to an entity formed by affiliates of Apollo Management, L.P. (Apollo) of New York City. The new company and U. S. Steel plan to enter into long-term contracts to supply U. S. Steel with its domestic iron ore and coke requirements and to provide U. S. Steel with transportation services.

ISG is the second largest steelmaking company in the United States. It was formed in 2002 by acquiring Bethlehem Steel, Weirton Steel, LTV Steel, and Georgetown Steel. It operates integrated steelmaking, EAF steelmaking, iron ore mining and processing, coking, scrap steel processing, DRI production, and logistics services. The logistics services consist of 1) eight short line railroads located in Pennsylvania, Maryland, New York, and Indiana; 2) lake shipping; and 3) trucking operations.

MW heating shows great advantages in decreasing iron ore reduction time. MW assisted direct reduced iron (DRI) production is a promising area of utilizing MW for steelmaking. DRI is replacement of scrap steel as EAF feed. Five DRI plants have been built in the United States as shown in Table 4.2. These DRI plants were originally independent operations supplying DRI to mini mills. Now, four of them are idle. Only Georgetown Steel is still in operation. Georgetown Steel was acquired by ISG in 2004.

\begin{tabular}{|l|l|l|c|c|}
\hline \multicolumn{5}{|c|}{ Table 4.2. US Direct Reduction (DRI) Plants } \\
\hline Plant & Process & Location & Capacity, Mt/y & Start-up \\
\hline Georgetown Steel & Midrex & Georgetown, SC & 0.40 & 1971 \\
\hline Corus Mobile & Midrex & Mobile, AL & 0.80 & $1997 / 1998$ \\
\hline American Iron Reduction & Midrex & Convent, LA & 1.20 & 1998 \\
\hline Qualitech & Iron Carbide & Corpus Christi, TX & 0.66 & 1999 \\
\hline Iron Dynamics & Iron Dynamics & Butler, IN & 0.50 & 1998 \\
\hline
\end{tabular}

Generally speaking, many steelmaking plants are located by harbors, lake shores and rivers in Illinois, Indiana, Ohio, and Pennsylvania. The raw materials, iron ore and coke, are normally shipped by barges up rivers and lakes, or transported by railroad from iron ore mines and coking plants to steelmaking plants. Finished steel products are commonly transported by trucks.

\section{Potential changes to interactions and logistics}

Now let's discuss the potential changes to steel company and supplier company interaction and logistics assuming the MW assisted steelmaking technology is successfully developed and used by the steel industry. We see three potential scenarios. 


\section{Scenario 1. Deployment of MW assisted DRI production at mines.}

Currently iron ore processors ship iron ore pellets as their end products to integrated steelmakers. The technology of MW assisted DRI production provides an opportunity for iron ore processors to produce DRI as their end product with much higher value. A change from making iron ore pellets to DRI will take time to accomplish simply because of the huge volume of material. The iron ore processors could start MW assisted DRI production on a small scale and gradually increase its share. In this scenario, the iron ore processors will not only supply iron ore pellets to integrated steelmakers but also supply DRI to mini mills. The iron ore processors could be independent entities which supply the raw material based on contracts. Or the iron ore processors could be wholly or partially owned by integrated steelmakers or mini mills. Logistics could be a part of the iron ore processor's business or arranged with independent or partially owned logistics companies based on supply contracts.

The potential problems of this scenario are the electricity, coal and limestone supplies. The MW assisted DRI production requires new electricity, coal and limestone supplies, which may not be readily available at the current iron ore mining operations. Current pelletizing technology generally uses natural gas as the fuel to heat iron ore agglomerates to $2400^{\circ} \mathrm{F}$. These changes may create barriers for an iron ore processor to use this technology.

Scenario 2. Deployment of MW assisted DRI production by independent DRI producers.

The MW assisted DRI production technology could be utilized by independent DRI producers. It may happen when the technology is proven economically viable but the dramatic change is too risky for mini mills. In such circumstances, mini mills will be reluctant to deploy MW assisted DRI facilities at their sites. It is something like the current DRI plants in the United States. These DRI plants were originally built by independent entities. The mini mills need stronger reasons to get involved in direct DRI production.

In this scenario, independent DRI producers may be more flexible and emerge to take the initiative. The independent DRI producers will select locations convenient to an iron ore processor and a mini mill, and having abundant electricity. The independent DRI producers will purchase iron ore concentrate, coal and fluxing agent. The independent DRI producer may need to set up pelletizing facilities and do its own pelletizing. The independent DRI producer will sell DRI to mini mills at a competing price.

We can envision some potential barriers in this scenario. Shipping iron ore concentrate and setting up agglomeration facilities are not very desirable to an independent DRI producer. One solution would be for the iron ore processor to mix and agglomerate pellets using their current facilities and ship the green pellets to independent DRI producers. In such an arrangement, the independent DRI producer will focus on MW assisted DRI reduction and supply interaction with mini mills. 
Scenario 3. Deployment of MW assisted DRI production or direct steelmaking at mini mills.

If this technology matures and demonstrates significant cost saving, or if scrap steel is in critical shortage, mini mills may have great interest in integrating MW assisted DRI production with EAF smelting or to deploy MW assisted direct steelmaking at their locations. In this circumstance, DRI will be produced separately and charged into EAFs directly or iron ore will be reduced and melted in a single MW/EAF. Either case will eliminate DRI cooling and further increase energy efficiency.

The required mixing and agglomeration operations of iron ore concentrate could be done either at an iron ore processor's site or at a mini mill site. The advantages of mixing and agglomeration at an iron ore processor's site will lower the investment and simplify the operation of the mini mill related to the DRI production. Mixing and agglomeration at a mini mill site may have the advantage of utilizing oxide scale as a part of feed.

\section{Conclusion}

Iron ore has the dominant quantity among steelmaking raw materials. In the current steelmaking operations, iron ore is mined and processed to produce pellets at mine sites using comparatively small amounts of electricity and large amounts of natural gas as fuel. The end product, iron ore pellets, are shipped to integrated mills by barge and rail with loading, unloading and storage facilities suitable for handling pellets. The changes caused by utilizing MW assisted steelmaking technology may either require shipping iron ore concentrate to locations away from mines or doing DRI production at mine sites. The first situation requires the adjustment of logistics from transportation and handling pellets to concentrates. The second situation requires establishment of additional electricity supply to the mine sites.

To avoid the above two situations, iron ore processors could conduct agglomeration of iron ore concentrates with additives of pulverized coal and limestone at mine sites and ship green pellets to independent DRI production sites or mini mills. 


\section{Task 5a - Energy Assessment}

This chapter consists of four sections: general energy analysis, minimum theoretical energy requirement, experimental energy consumption, and energy consumption comparison between $\mathrm{MW} / \mathrm{EAF}$ steelmaking process and conventional steelmaking processes.

\subsection{General Energy Analysis}

The general energy analysis is to analysis the energy consumption of MW/EAF process based on the raw materials used in the bench steelmaking tests described in Section 3 of Chapter 1.

Energy input into MW/EAF process includes electric energy used for microwave heating and electric arc heating and coal blended into the iron ore pellets. A part of energy in the coal is in the volatiles. The volatiles complete escape at a temperature lower than the temperature required for iron ore reduction. We assume no volatile participating in iron ore reduction in this analysis. The energy contained in the fixed carbon of the coal is consumed by iron oxide reduction, combustion with oxygen and remaining in the produced steel. Since the carbon content of steel is fairly low, we assume no carbon in the produced metal to simplify the energy analysis.

Actual iron ores may consist of magnetite $\left(\mathrm{Fe}_{3} \mathrm{O}_{4}\right)$, hematite $\left(\mathrm{Fe}_{2} \mathrm{O}_{3}\right)$ and wustite $(\mathrm{FeO})$. This analysis only considers magnetite iron ore.

The heating sources of MW/EAF process include microwave heating, electric arc heating and exothermic reactions. The exothermic reactions include combustion of volatiles, combustion of fixed carbon with oxygen, and other carbon involved exothermic reactions.

Specific energy consumption $(\mathrm{MJ} / \mathrm{kg}$ ) indicates the amount of energy is consumed to produce a given amount of hot metal. Specific energy consumption is used in this chapter to compare the energy efficiency among steelmaking processes.

As the energy consumption of MW/EAF process can vary in a wide range with different pellet compositions and operation conditions, it is of practical interest to calculate theoretical energy consumption. From the calculation, the most important factors that have effects on the energy consumption of MW/EAF process will be included. Based on the analysis, the energy efficiency can be improved by selecting appropriate experimental settings.

\section{Nomenclature}

$\begin{array}{ll}t_{\mathrm{MW}} & \text { Microwave heating time [second], otherwise specified } \\ t_{\mathrm{EAF}} & \text { Electric arc heating time [second], otherwise specified } \\ \mathrm{P}_{\mathrm{MW}} & \text { Microwave output power [watt] } \\ \mathrm{I} & \text { Electric current of electric arc heating [ampere] } \\ \mathrm{V} & \text { Applied voltage of electric arc heating [volt] } \\ \mathrm{W} & \text { Weight of pellet sample [gram] } \\ \mathrm{W}_{\mathrm{Fe}} & \text { Weight of produced steel [gram] } \\ \mathrm{C}_{\mathrm{cm}} & \text { Weight ratio of coal to magnetite ore with weight } 100 \text { in pellet sample }\end{array}$




$\begin{array}{ll}C_{c m}^{\min } & \begin{array}{l}\text { Minimum weight ratio of coal to magnetite with weight } 100 \text { in pellet } \\ \text { sample for completely reducing magnetite }\end{array} \\ \mathrm{C}_{\mathrm{bm}} & \text { Weight ratio of binder to magnetite ore with weight } 100 \text { in pellet sample } \\ \mathrm{C}_{l \mathrm{~m}} & \text { Weight ratio of lime to magnetite ore with weight } 100 \text { in pellet sample } \\ \mathrm{C}_{\mathrm{v}} & \text { Volatile materials content of coal [wt \%] } \\ \mathrm{C}_{\mathrm{c}} & \text { Fixed carbon content of coal [wt } \% \text { ] } \\ \mathrm{C}_{\mathrm{m}} & \text { Magnetite content of magnetite ore [wt } \% \text { ] } \\ \mathrm{Y} & \text { Steel yield [\%] } \\ \mathrm{S}_{\mathrm{E}} & \text { Specific energy consumption of steel produced }[\mathrm{kJ} / \mathrm{kg}] \\ \mathrm{E} & \text { Energy [joules] } \\ \mathrm{T} & \text { Temperature }[\mathrm{K}] \\ \mathrm{C}_{\mathrm{p}} & \text { Heat capacity of materials [joules } / \text { mole } \mathrm{K}] \\ \Delta \mathrm{H}_{\text {trans }} & \text { Molar heat of phase transformation [joules } / \mathrm{mole}] \\ \Delta \mathrm{G} & \text { Free energy change of chemical reaction }[\mathrm{joules} / \mathrm{mole}]\end{array}$

\section{Temperature Change in MW/EAF Process}

Assuming steelmaking is performed using a single MW/EAF furnace as we did in the bench steelmaking tests. The raw material pellets are made of coal, magnetite ore, lime and binder. The pellets are loaded into a crucible placed in MW/EAF furnace at room temperature. Microwave is introduced into the furnace for a while to heat the pellets. Electrical arcing is then turned on to

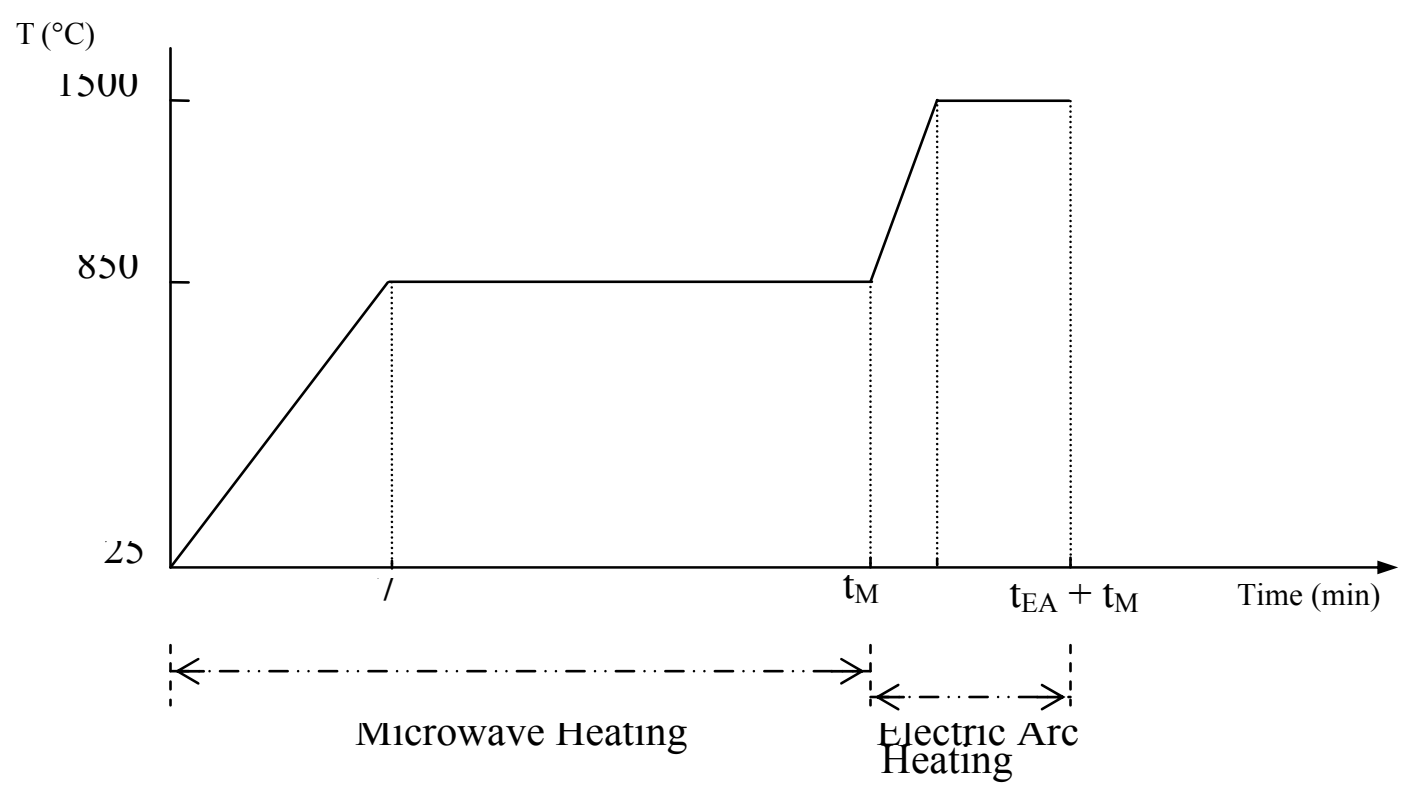

Figure 5.1 Variation of burden temperature in MW/EAF process

heat the burden until steel is produced. The burden's temperature is a useful parameter to calculate the energy consumption. Exact temperature curve of the burden is not available due to limitation of measuring tools and difficulty of measurement under the tough environment of MW and EAF heating. However, the burden's temperatures at some critical points in MW/EAF process were measured. Based on these data at critical points, the relationship between the burden's temperature and time in the process is established as shown in Figure 5.1. 


\section{Thermodynamic Analysis of Chemical Reactions}

To calculate the energy consumption of MW/EAF process, the thermodynamic data about the free-energy changes of the chemical reactions in MW/EAF process should be calculated first. The equation to calculate free energy change of chemical reaction is:

$$
\Delta G=\Delta H_{298}+\int_{298}^{T} \Delta C_{p} d t-T \Delta S_{298}-T \int_{298}^{T} \frac{\Delta C_{p}}{t} d t
$$

Where $\Delta \mathrm{H}_{298}$ and $\Delta \mathrm{S}_{298}$ are the change of formation heats and entropies between products and reactants at ambient temperature respectively, $\Delta \mathrm{C}_{\mathrm{p}}$ is the change of heat capacities between products and reactants.

The main reactions and their free-energy change in MW/EAF process are ${ }^{[22]}$ :

$$
\begin{gathered}
\mathrm{C}_{(s)}+\mathrm{O}_{2(g)}=\mathrm{CO}_{2(g)} \\
\Delta \mathrm{G}=-394472.4-0.8375 \mathrm{~T} \\
\mathrm{CO}_{2(g)}+\mathrm{C}_{(s)}=2 \mathrm{CO}_{(g)} \\
\Delta \mathrm{G}=170854.3-174.6 \mathrm{~T} \\
\mathrm{Fe}_{3} \mathrm{O}_{4(s)}+4 \mathrm{CO}_{(g)}=3 \mathrm{Fe}_{(s)}+4 \mathrm{CO}_{2(g)} \\
\Delta \mathrm{G}=-22822.45+26.13 \mathrm{~T} \\
\mathrm{CH}_{4(g)}+2 \mathrm{O}_{2(g)}=\mathrm{CO}_{2(g)}+2 \mathrm{H}_{2} \mathrm{O}_{(g)} \\
\Delta \mathrm{G}=-299384+59.9144 \mathrm{~T}+0.0145 \mathrm{~T}^{2}+234240 \mathrm{~T}^{-1}-7.66 \mathrm{~T} \ln (\mathrm{T}) \\
\mathrm{Fe}_{3} \mathrm{O}_{4(s)}+4 \mathrm{C}_{(s)}+2 \mathrm{O}_{2(g)}=3 \mathrm{Fe}_{(s)}+4 \mathrm{CO}_{2(g)} \\
\Delta \mathrm{G}=-470058.6-324.79 \mathrm{~T}
\end{gathered}
$$

Carbon inside coal serves two functions in MW/EAF process. A part of carbon acts as reducing agent and the rest acts as fuel and produces heat. Fuel carbon reacts only with oxygen as indicated by chemical equation 5-2 and produces carbon dioxide which is released out of the furnace. Carbon as reductant participates in chemical reactions 5-2, 5-3 and 5-4, and reduces magnetite to iron. Chemical reactions 5-2,5-3 and 5-4 can be considered as overall chemical reaction 5-6. Although "Boudouard" reaction 5-3 is endothermic and only proceeds at least above $400{ }^{\circ} \mathrm{C}$, overall reaction 5-6 is an exothermic reaction, so carbon as reducing agent also produces heat.

Volatile materials inside coal can be represented by molecular formula $\left(\mathrm{CH}_{4}\right)_{\mathrm{m}}$. Due to the volatile characteristic of these materials, they stay in the early stage of MW/EAF process, while 
the inner temperature of furnace is not high. Volatile materials are oxidized as indicated by chemical equation 5-5 if oxygen is present.

The free-energy changes of chemical reactions 5-2 and 5-5 are dependent on the temperature at which the reactions proceed. Although the temperature change in MW/EAF process is established, the reaction rates of these reactions are unknown. We have to approximate the freeenergy changes of those chemical reactions using some values at special temperature.

Chemical reaction 5-5 can occur at relatively low temperature. The free-energy change of chemical reaction 5-5 varies slightly with temperature, while temperature is below $1000{ }^{\circ} \mathrm{C}$. The value of free energy change at $700^{\circ} \mathrm{C}$ is $277.6 \mathrm{~kJ} / \mathrm{mole}$.

The variation of free-energy change vs temperature of chemical reaction 5-2 is small in the temperature range of MW/EAF process, due to the small slope. The overall chemical reaction 56 proceeds rapidly at an elevated temperature. It is appropriate to calculate the free energy change of reaction 5-2 at temperature $700{ }^{\circ} \mathrm{C}$. The free energy change of the reaction is 395.8 $\mathrm{kJ} /$ mole, very significant. The chemical reaction 5-4 is exothermic at lower temperatures and becomes endothermic at higher temperatures. It absorbs $6.5 \mathrm{~kJ} / \mathrm{mole}$ energy at $850^{\circ} \mathrm{C}$.

\section{Energy Balance}

The energy balance of MW/EAF process can be expressed by equation 5-7. The heating energies to the process in the left side of the equation consist of electric energy and chemical energies released from coal. According to the analysis in section 5.1.2., all carbon and volatile materials in coal produce heat except "Boudouard" reaction 5-3 is endothermic.

$E_{\text {EAF }}+E_{M W}+E_{\text {exothermic }}=E_{\text {endothermic }}+E_{\text {vapor }}+E_{\text {magnetite }}+E_{\text {oarbon }}+E_{\text {slag }}+E_{\text {steel }}+E_{\text {offgas }}+E_{\text {losses }}$

The energies absorbed by the process in the right side of the equation include the energies required by the process and energy losses. The components in the raw materials and the end products are grouped into magnetite, carbon, slag and steel. The slag includes $\mathrm{CaO}, \mathrm{SiO}_{2}$ and their reaction product: $\mathrm{CaSiO}_{3} . E_{\text {magnetite }}$ and $E_{\text {carbon }}$ represent the energies absorbed by magnetite and fixed carbon to reach a temperature before reduction or a chemical reaction takes place.

$E_{\text {losses }}$ include energies absorbed by the crucible and surrounding fireclay bricks as well as conversion losses from electricity to $\mathrm{MW}$ and from electricity to arcing. The energies absorbed by the furnace and surrounding environment are unnecessary to MW/EAF process. Although it is impossible to eliminate the energy consumption of refractory and surrounding environment, decreasing the ratio of this energy consumption can greatly improve the energy efficiency of the direct steelmaking technology. 


\section{Specific Energy Consumption}

It is difficult to calculate the total energy consumption by considering all the energy-consuming factors due to heat loss to the surrounding environment. Based on the fact that the sum of input electric energy and released energy from exothermic reactions is equal to the total energy consumption in MW/EAF process, energy consumption can be formulated by considering input electric energy and exothermic heat.

Assume there is enough coal in the pellets to reduce the magnetite and the magnetite is completely reduced to iron.

The weight of iron in pellets $\mathrm{W}$ is $\frac{168 * 100 * W C_{m}}{232 *\left(100+C_{c m}+C_{l m}+C_{b m}\right)}$.

Given steel yield Y, the weight of produced steel is $\frac{168 * 100 * Y W C_{m}}{232 *\left(100+C_{c m}+C_{l m}+C_{b m}\right)}$.

The molar number of volatile material in pellets is $\frac{W C_{c m} C_{v}}{16^{*}\left(100+C_{c m}+C_{l m}+C_{b m}\right)}$ mole. Combustion of volatile material in coal is represented by reaction 5-5, that is exothermic and the molar heat at temperature $700{ }^{\circ} \mathrm{C}$ is 278399 joules/mole. The heat released from volatile materials combustion is $\frac{W C_{c m} C_{v}}{16 *\left(100+C_{c m}+C_{l m}+C_{b m}\right)} * 278399$ joules.

If there is abundant fixed carbon in pellets, it will combust and release heat. The molar number of abundant carbon is $\frac{W}{100+C_{c m}+C_{l m}+C_{b m}}\left(\frac{C_{c m} C_{c}}{12}-\frac{2 * 100 * C_{m}}{232}\right)$ mole. The molar heat of reaction 5-2 at temperature $700{ }^{\circ} \mathrm{C}$ is 395800 joules/mole, so the heat released from abundant fixed carbon combustion is

$\frac{W}{100+C_{c m}+C_{l m}+C_{b m}}\left(\frac{C_{c m} C_{c}}{12}-\frac{2 * 100 * C_{m}}{232}\right) * 395800$ joules.

The sum of energies released from exothermic reaction is

$\frac{W}{100+C_{c m}+C_{l m}+C_{b m}}\left(\frac{395800 * C_{c m} C_{c}}{12}+\frac{278399 C_{c m} C_{v}}{16}\right)$ joules.

The energies input through microwave heating and electric arc heating are $t_{\mathrm{MV}} * \mathrm{P}_{\mathrm{MV}}$ joules and $\mathrm{t}_{\mathrm{EAF}} * \mathrm{I} * \mathrm{~V}$ joules respectively.

The specific energy consumption in $\mathrm{kJ} / \mathrm{kg}$ is:

$$
\frac{\frac{t_{M V} P_{M V}+t_{E A F} I^{*} V}{1000}+\frac{W}{100+C_{c m}+C_{l m}+C_{b m}}\left(\frac{395.8 * C_{c m} C_{c}}{12}+\frac{278.40 C_{c m} C_{v}}{16}\right)}{\frac{168 * 100 * Y W C_{m}}{1000 * 232 *\left(100+C_{c m}+C_{l m}+C_{b m}\right)}}
$$


Thus equation 5-8 represents the specific energy consumption of the new steal making process.

$$
S_{E}=\frac{0.01381 *\left(t_{M V} P_{M V}+t_{E A F} I V\right)\left(100+C_{c m}+C_{l m}+C_{b m}\right)}{Y W C_{m}}+\frac{\left(455.5 C_{c m} C_{c}+239.65 C_{c m} C_{v}\right)}{Y C_{m}}
$$

\section{Minimum Coal to Magnetite Ratio for Complete Reduction}

In the above computation, we assume there is enough coal in pellets to completely reduce magnetite into iron. Though, what is the minimum ratio of coal to magnetite ore in pellets required for complete reduction? The minimum coal ratio is also a useful reference to increase the energy efficiency of MW/EAF process by decreasing the usage of coal.

Because volatile materials in coal can not reduce magnetite, if all the fixed carbon in coal serves as reducing agent, the amount of coal in pellets is the minimum ratio for complete reduction.

The molar number of magnetite in the pellets is $\frac{100 W C_{m}}{232 *\left(100+C_{c m}+C_{l m}+C_{b m}\right)}$ mole.

The molar number of fixed carbon inside the pellets is $\frac{W C_{c m} C_{c}}{12 *\left(100+C_{c m}+C_{l m}+C_{b m}\right)}$ mole.

Although equation 5-6 indicates that one mole of carbon only can reduce 1/4 mole of magnetite, actually one mole of carbon can reduce $1 / 2$ mole of magnetite if the production carbon dioxide from reaction 5-4 takes part in the "Boudouard" reaction. Thus $\frac{W C_{c m} C_{c}}{12 *\left(100+C_{c m}+C_{l m}+C_{b m}\right)}$ mole of fixed carbon in pellet sample can reduce $\frac{W C_{c m} C_{c}}{2 * 12 *\left(100+C_{c m}+C_{l m}+C_{b m}\right)}$ mole of magnetite. Assume magnetite in pellets is completely reduced, we have the following equation:

$$
\frac{100 C_{m}}{232}=\frac{C_{c m} C_{c}}{2 * 12} .
$$

Thus the minimum coal ratio in pellets is:

$$
C_{c m}^{\min }=\frac{10.34 C_{m}}{C_{c}}
$$

The minimum ratios of coals with different amount of fixed carbon are summarized in Table 5.1 as examples.

Table 5.1. Minimum ratios of different coals to magnetite ore with $81.2 \%$ magnetite

\begin{tabular}{|c|c|c|c|}
\hline Fixed carbon of coal (\%) & 59.86 & 72.72 & 88.2 \\
\hline$C_{c m}^{\min }$ & 14.03 & 11.55 & 9.52 \\
\hline
\end{tabular}




\subsection{Minimum Theoretical Energy Consumption}

\section{Basic Assumption and Equations}

The calculations performed are theoretical. The basic assumptions are given as following:

(1) Only energy directly used in MW/EAF process is considered.

(2) Energy and heat losses to furnace, refractory and electrical equipment are excluded.

(3) Energy credits in the offgas are excluded.

(4) Energy loss due to yield loss is not considered.

(5) Assume that yield is Y.

(6) Effects of steel composition on energy consumption are not considered.

(7) Heating temperature is a variable in MW/EAF process. Assume that final smelting temperature is $1700^{\circ} \mathrm{C}$.

(8) Energy for reduction of silica and manganese oxide is excluded.

(9) Assume the coal content of pellets is the minimum coal required for complete reduction.

(11) Assume typical slag basicity is 1.2.

(12) Assume the energy balance is

$$
E_{E A F}+E_{M W}+E_{\text {exothermic }}=E_{\text {endothermic }}+E_{\text {vapor }}+E_{\text {slag }}+E_{\text {steel }}+E_{\text {offgas }}
$$

Where $E_{E A F}$ and $E_{M W}$ are input energies through electric arc heating and microwave heating; $E_{\text {exothermic }}$ is heat released from exothermic chemical reactions; $E_{\text {endothermic }}$ is heat absorbed by endothermic chemical reactions; $E_{\text {vapor }}$ and $E_{\text {offgas }}$ are heat absorbed by water vapor and offgas; $E_{\text {slag }}$ and $E_{\text {steel }}$ are heat associated with slag and steel.

Changes in state functions do not depend on the details of a process but only on the initial and final states, so the methodology for calculating theoretical energy consumption is that all chemical reactions occur at ambient temperature $\left(298^{\circ} \mathrm{K}\right)$ and products are heated to the temperature of final states. Therefore, the energies absorbed by magnetite and coal to their reaction temperatures are omitted.

The energy $E$ for heating materials is given by:

$$
E=\int_{T_{1}}^{T_{2}} n C_{p} d T
$$

Where $C_{p}$ is heat capacity of materials depending on temperature, $T_{1}$ and $T_{2}$ are the initial and final temperature, $\mathrm{n}$ is the number of moles.

The energy $E$ associated with phase transformation is given by:

$$
E=n \Delta H_{\text {trans }}
$$

Where $\Delta H_{\text {trans }}$ is the molar heat of phase transformation, $\mathrm{n}$ is the number of moles. 


\section{Minimum Theoretical Energy Consumption Formulas}

If there is abundant fixed carbon in coal, it will burn to carbon dioxide and release heat as fuel, as represented by reaction 5-2. The molar heat of reaction 5-2 at ambient temperature is 394722 joules/mole, so the heat released from abundant fixed carbon combustion is:

$$
E_{\text {carbon-combustion }}=\frac{W\left(C_{c m}-C_{c m}^{\min }\right) C_{c}}{12 *\left(100+C_{c m}+C_{l m}+C_{b m}\right)} * 394722
$$

The combustion of volatile material in coal is represented by reaction 5-5, which is exothermic. The molar heat at ambient temperature is 292460 joules/mole, so the heat released from volatile materials combustion is:

$$
E_{\text {volatile-combustion }}=\frac{W C_{c m} C_{v}}{16 *\left(100+C_{c m}+C_{l m}+C_{b m}\right)} * 292460
$$

The specific heat of starch is 15.2 joules/g, so heat released from starch combustion is:

$$
E_{\text {starch-combustion }}=\frac{W C_{b m}}{\left(100+C_{c m}+C_{l m}+C_{b m}\right)} * 15.2
$$

Reaction 5-4 is exothermic at lower temperatures; the molar heat at ambient temperature is 15035 joules/mole. The heat released from magnetite reduction is:

$$
E_{\text {reduction }}=\frac{100 * W C_{m}}{232 *\left(100+C_{c m}+C_{l m}+C_{b m}\right)} * 15035
$$

The total energy released from exothermic reactions is:

$$
E_{\text {exothermic }}=E_{\text {carbon-combustion }}+E_{\text {volatile-combustion }}+E_{\text {starch-combustion }}+E_{\text {reduction }}
$$

The "Boudouard" reaction 5-3 is endothermic reaction and the molar heat at ambient temperature is 118817 joules/mole, so the heat required to produce carbon monoxide is:

$$
E_{\text {endothermic }}=\frac{W C_{c m}^{\min } C_{c}}{12 *\left(100+C_{c m}+C_{l m}+C_{b m}\right)} * 118817
$$

The heat capacities of $\mathrm{SiO}_{2}$ and $\mathrm{CaO}$ are $C_{P\left(\mathrm{SiO}_{2}\right)}=44.77$ joules $/ \mathrm{mole}{ }^{\circ} \mathrm{K}$ and $C_{P(\mathrm{CaO})}=42$ joules $/$ mole $^{\circ} \mathrm{K}$. Given $\mathrm{W}$ gram pellets, the theoretical energies used to heat the components of the slag from ambient temperature to temperature of $\mathrm{T}$ are:

$$
E_{\mathrm{CaO}}=n_{\mathrm{CaO}} \int_{298}^{T} C_{p(\mathrm{CaO})} d T=\frac{100 W\left(1-C_{m}\right)+W C_{l m}+W C_{c m}\left(1-C_{v}-C_{c}\right)}{1.83 * 56 *\left(100+C_{c m}+C_{l m}+C_{b m}\right)} * 42(T-298)
$$




$$
E_{\mathrm{SiO}_{2}}=n_{\mathrm{SiO}_{2}} \int_{298}^{T} C_{p\left(\mathrm{SiO}_{2}\right)} d T=\frac{100 W\left(1-C_{m}\right)+W C_{l m}+W C_{c m}\left(1-C_{v}-C_{c}\right)}{2.2 * 60 *\left(100+C_{c m}+C_{l m}+C_{b m}\right)} * 44.77(T-298)
$$

The endothermic reaction forming slag is:

$$
\begin{aligned}
\mathrm{CaO}+\mathrm{SiO}_{2}=\mathrm{CaSiO}_{3} \\
\\
\Delta \mathrm{G}=92500+2.5 \mathrm{~T}(\mathrm{~J})
\end{aligned}
$$

The heat associated with forming of the slag is:

$$
E_{\mathrm{CaSiO}_{2}}=\frac{100 W\left(1-C_{m}\right)+W C_{l m}+W C_{c m}\left(1-C_{v}-C_{c}\right)}{2.2 * 60 *\left(100+C_{c m}+C_{l m}+C_{b m}\right)} *(92500+2.5 T)
$$

The total energy absorbed by slag is:

$$
E_{\text {slag }}=E_{\mathrm{CaO}}+E_{\mathrm{SiO}_{2}}+E_{\mathrm{CaSiO}_{3}}
$$

The heat capacities of $\alpha-\mathrm{Fe}$ and $\gamma$-Fe are $C_{P(\alpha-F e)}=17.5+24.8 \times 10^{-3} T$ joules $/ \mathrm{mole}{ }^{\circ} \mathrm{K}$ and $C_{P(\gamma-F e)}=7.7+19.5 \times 10^{-3} \mathrm{~T}$ joules $/ \mathrm{mole}^{\circ} \mathrm{K}$. The temperature of phase transformation from $\alpha-\mathrm{Fe}$ to $\gamma$-Fe is $1181^{\circ} \mathrm{K}$. The theoretical energies used to heat iron from ambient temperature to temperature of $\mathrm{T}$ are:

$$
\begin{aligned}
& E_{\alpha-F e}=n_{F e} \int_{298}^{1181} C_{p(\alpha-F e)} d T=\frac{3 * 100 * W C_{m}}{232 *\left(100+C_{c m}+C_{l m}+C_{b m}\right)} * 47840 \\
& E_{\gamma-F e}=n_{F e} \int_{1181}^{T} C_{p(\alpha-F e)} d T=\frac{3 * 100 W C_{m}}{232 *\left(100+C_{c m}+C_{l m}+C_{b m}\right)}\left(7.7(T-1181)+19.5 \frac{T^{2}-1181^{2}}{1000}\right)
\end{aligned}
$$

The heat associated with solid phase transformation from $\alpha$-Fe to $\gamma$-Fe is:

$$
E_{\alpha \rightarrow \gamma}=\frac{3 * 100 * W C_{m}}{232 *\left(100+C_{c m}+C_{l m}+C_{b m}\right)} * 5940
$$

The heat associated with phase transformation from $\gamma$-Fe to liquid $\mathrm{Fe}$ is:

$$
\begin{aligned}
& E_{\gamma \rightarrow l}=\frac{3 * 100 * W * C_{m}}{232 *\left(100+C_{c m}+C_{l m}+C_{b m}\right)} *(13800+7.6 * T) \\
& E_{l}=\frac{3 * 100 * W * C_{m}}{232 *\left(100+C_{c m}+C_{l m}+C_{b m}\right)} * 41.88 *\left(T_{\text {disch arge }}-T\right)
\end{aligned}
$$


The energy absorbed by steel is:

$$
E_{\text {steel }}=E_{\alpha-F e}+E_{\gamma-F e}+E_{\alpha \rightarrow \gamma}+E_{\gamma \rightarrow l}+E_{l}
$$

Water vapor comes from the combustion of starch and volatile materials inside coal. Because one carbon atom usually binds with two hydrogen atoms on a long chain of atoms in organic materials such as starch, it is reasonable to represent starch by molecule formula $\left(\mathrm{CH}_{2}\right)_{\mathrm{m}}(\mathrm{OH}) \mathrm{n}$ (where $\mathrm{n}<<\mathrm{m}$ ). The volatile materials can be represented by $\mathrm{CH}_{4}$.

The heat capacity of water vapor is $C_{p\left(H_{2} O\right)}=30+10.7 \times 10^{-3} T+0.33 \times 10^{5} T^{-2}$ joules $/ \mathrm{mole}{ }^{\circ} \mathrm{K}$. The theoretical energies used to heat water vapor from ambient temperature to temperature of $\mathrm{T}$ is:

$$
\begin{aligned}
&\left.E_{\mathrm{H}_{2} \mathrm{O}}=n_{\mathrm{H}_{2} \mathrm{O}} \int_{298}^{T} C_{p\left(\mathrm{H}_{2} \mathrm{O}\right.}\right) d T=\frac{W}{\left(100+C_{c m}+C_{l m}+C_{b m}\right)} * \\
&\left(\frac{2 C_{c m} C_{v}}{16}+\frac{C_{b m}}{14}\right)\left(30(T-298)+0.00107\left(T^{2}-298^{2}\right)+0.33 \times 10^{5}\left(\frac{1}{T}-\frac{1}{298}\right)\right)
\end{aligned}
$$

The phase transformation of water is represented by following equation:

$$
\begin{aligned}
& \mathrm{H}_{2} \mathrm{O}(\text { liquid }) \rightarrow \mathrm{H}_{2} \mathrm{O}(\text { gas }) \\
& \Delta \mathrm{G}=40650(\mathrm{~J})
\end{aligned}
$$

The heat associated with evaporation of water is:

$$
E_{H_{2} O(l \rightarrow g)}=\frac{W}{\left(100+C_{c m}+C_{l m}+C_{b m}\right)} *\left(\frac{2 * C_{c m} C_{v}}{16}+\frac{C_{b m}}{14}\right) * 40650
$$

The total energy absorbed by water vapor is:

$$
E_{\text {vapor }}=E_{\mathrm{H}_{2} \mathrm{O}}+E_{\mathrm{H}_{2} \mathrm{O}(l \rightarrow g)}
$$

The heat capacity of $\mathrm{CO}_{2}$ is 36.62 joules $/ \mathrm{mole}^{\circ} \mathrm{K}$. The theoretical energies used to heat carbon dioxide from ambient temperature to temperature of $\mathrm{T}$ is:

$$
\left.E_{\text {offgas }}=n_{C O_{2}} \int_{298}^{T} C_{p\left(C O_{2}\right.}\right) d T=\frac{36.62 * W(T-298)}{\left(100+C_{c m}+C_{l m}+C_{b m}\right)}\left(\frac{C_{c m} C_{v}}{16}+\frac{C_{c m} C_{c}}{12}+\frac{C_{b m}}{14}\right)
$$

Equation 5-35 represents the steel weight produced from pellets of weight $\mathrm{W}$, assuming that yield is Y. Specific energy consumption can be calculated through dividing energy consumption by steel weight.

$$
W_{F e}=\frac{168 * 100 * W Y C_{m}}{232 *\left(100+C_{c m}+C_{l m}+C_{b m}\right)}
$$




\section{Minimum Energy Consumption Computation}

Table 5.2 and Table 5.3 summarize the theoretical energy consumption of two pellet samples with high volatile coal and low volatile coal respectively. Because the coal ratios in the pellet samples are minimum, the energy consumption of the two pellet samples are minimum theoretical energy consumptions. Table 5.2 and Table 5.3 indicate that about $45 \%$ of the total energy is consumed by steel, $22 \%$ to $28 \%$ is absorbed by water vapor and offgas. The percentage of energy consumed by water vapor and offgas in the total energy consumption is remarkable.

Total energy consumption significantly varies with the volatile content of coal. Pellet samples with high volatile coal need more energy to be reduced. The difference of total energy consumption between the two pellet samples mainly results from the energy absorbed by water vapor and offgas, because greater amounts high volatile coal are required for complete reduction and high volatile coal produces more water vapor.

Table 5.2 and Table 5.3 also indicate that the pellet sample with high volatile coal can release more heat due to combustion of volatile materials. For the two samples, the heats released from combustion of volatile materials are $504 \mathrm{~kJ}$ and $154 \mathrm{~kJ}$.

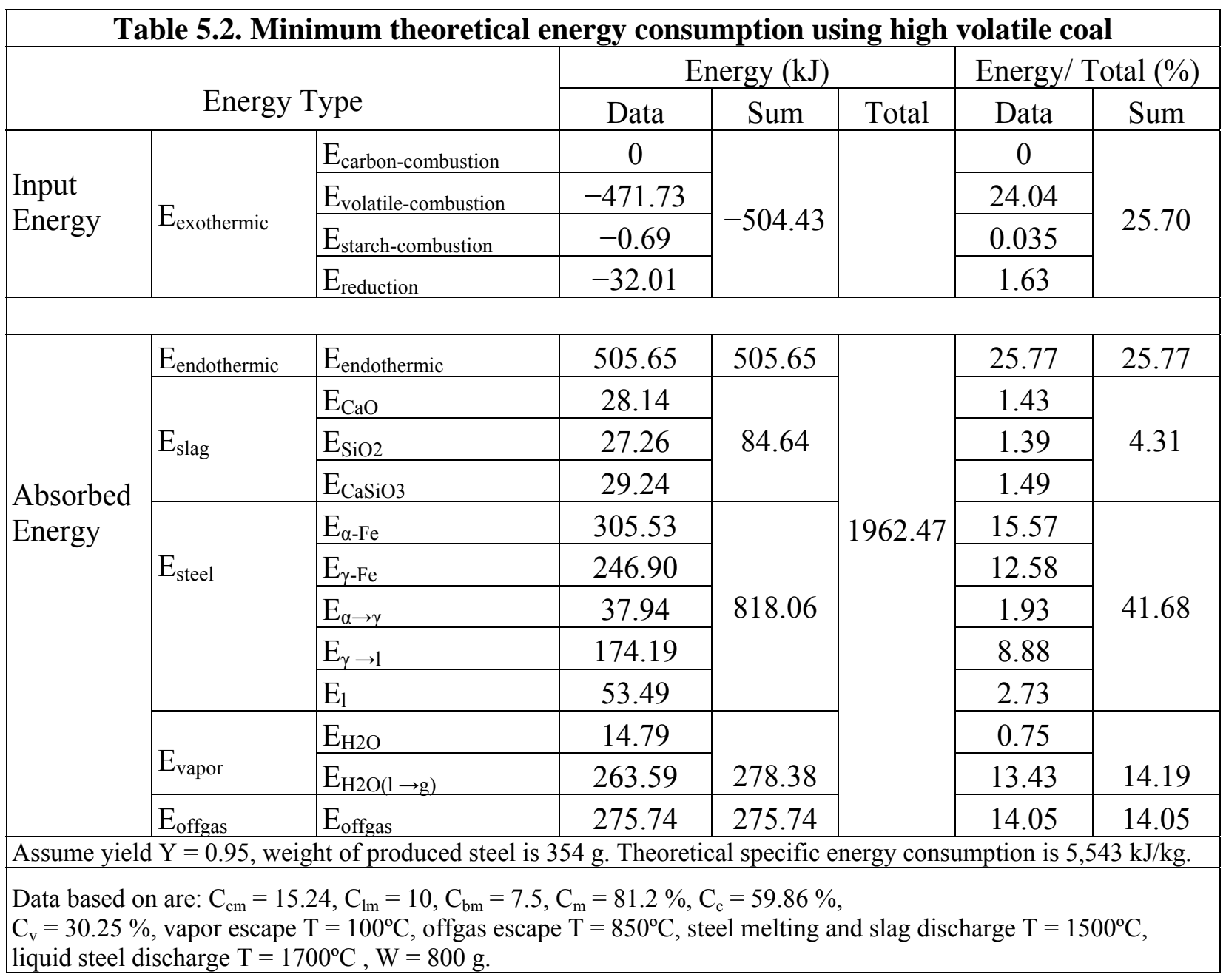




\begin{tabular}{|c|c|c|c|c|c|c|c|}
\hline \multicolumn{8}{|c|}{ Table 5.3. Minimum theoretical energy consumption using low volatile coal } \\
\hline \multirow{2}{*}{\multicolumn{3}{|c|}{ Energy Type }} & \multicolumn{3}{|c|}{ Energy $(\mathrm{kJ})$} & \multicolumn{2}{|c|}{ Energy/ Total $(\%)$} \\
\hline & & & \multirow{2}{*}{ Data } & Sum & Total & Data & Sum \\
\hline \multirow{4}{*}{$\begin{array}{l}\text { Input } \\
\text { Energy }\end{array}$} & \multirow{4}{*}{$\mathrm{E}_{\text {exothermic }}$} & $E_{\text {carbon-combustion }}$ & & \multirow{4}{*}{-154.18} & & 0 & \multirow{4}{*}{8.26} \\
\hline & & Evolatile-combustion $_{\text {. }}$ & -120.60 & & & 6.46 & \\
\hline & & Estarch-combustion $_{\text {S }}$ & -0.71 & & & 0.038 & \\
\hline & & $\mathrm{E}_{\text {reduction }}$ & -32.87 & & & 1.73 & \\
\hline & & & & & & & \\
\hline \multirow{12}{*}{$\begin{array}{l}\text { Absorbed } \\
\text { Energy }\end{array}$} & $\mathrm{E}_{\text {endothermic }}$ & $\mathrm{E}_{\text {endothermic }}$ & 519.33 & 519.33 & \multirow{12}{*}{1866.08} & 27.83 & 27.83 \\
\hline & \multirow{3}{*}{$E_{\text {slag }}$} & $\mathrm{E}_{\mathrm{CaO}}$ & 27.22 & \multirow{3}{*}{81.86} & & 1.46 & \multirow{3}{*}{4.39} \\
\hline & & $\mathrm{E}_{\mathrm{SiO} 2}$ & 26.50 & & & 1.42 & \\
\hline & & $\mathrm{E}_{\mathrm{CaSiO} 3}$ & 28.13 & & & 1.51 & \\
\hline & \multirow{5}{*}{$\mathrm{E}_{\text {steel }}$} & $\mathrm{E}_{\alpha-\mathrm{Fe}}$ & 313.80 & \multirow{5}{*}{840.19} & & 16.82 & \multirow{5}{*}{45.02} \\
\hline & & $\mathrm{E}_{\gamma-\mathrm{Fe}}$ & 253.58 & & & 13.59 & \\
\hline & & $E_{\alpha \rightarrow \gamma}$ & 38.96 & & & 2.09 & \\
\hline & & $\mathrm{E}_{\gamma \rightarrow 1}$ & 178.91 & & & 9.59 & \\
\hline & & $\mathrm{E}_{1}$ & 54.94 & & & 2.94 & \\
\hline & \multirow{2}{*}{$\mathrm{E}_{\text {vapor }}$} & $\mathrm{E}_{\mathrm{H} 2 \mathrm{O}}$ & 9.52 & \multirow[b]{2}{*}{179.08} & & 0.51 & \multirow[b]{2}{*}{9.60} \\
\hline & & $\mathrm{E}_{\mathrm{H} 2 \mathrm{O}(1 \rightarrow \mathrm{g})}$ & 169.56 & & & 9.09 & \\
\hline & $E_{\text {offgas }}$ & $\mathrm{E}_{\text {offgas }}$ & 245.61 & 245.61 & & 13.16 & 13.16 \\
\hline \multicolumn{8}{|c|}{ Assume yield $\mathrm{Y}=0.95$, weight of produced steel is $358 \mathrm{~g}$. Theoretical specific energy consumption is $5,213 \mathrm{~kJ} / \mathrm{kg}$. } \\
\hline \multicolumn{8}{|c|}{$\begin{array}{l}\text { Data based on are: } \mathrm{C}_{\mathrm{cm}}=10.56, \mathrm{C}_{\mathrm{lm}}=10, \mathrm{C}_{\mathrm{bm}}=7.5, \mathrm{C}_{\mathrm{m}}=81.2 \%, \mathrm{C}_{\mathrm{c}}=79.5 \% \text {, } \\
\mathrm{C}_{\mathrm{v}}=10 \% \text {, vapor escape } \mathrm{T}=100^{\circ} \mathrm{C} \text {, offgas escape } \mathrm{T}=850^{\circ} \mathrm{C} \text {, steel melting and slag discharge } \mathrm{T}=1500^{\circ} \mathrm{C} \text {, liquid } \\
\text { steel discharge } \mathrm{T}=1700^{\circ} \mathrm{C}, \mathrm{W}=800 \mathrm{~g} \text {. }\end{array}$} \\
\hline
\end{tabular}

The theoretical specific energy consumption of two pellet samples at steel discharge temperature $1700^{\circ} \mathrm{C}$ are $5.543 \mathrm{MJ} / \mathrm{kg}$ and $5.213 \mathrm{MJ} / \mathrm{kg}$ respectively. The difference between the quantities of steel yielded from the two pellet samples is slight, so the notable difference of theoretical specific energy consumption between the two samples mainly results from the different total energy consumption. For a pellet sample with high volatile coal, the energies absorbed by water vapor and offgas are increased by $55 \%$ and $12.3 \%$ with respect to that of the pellet sample with low volatile coal.

\subsection{Experimental Energy Consumption}

Energy efficiency of an industrial steelmaking furnace capable of 50-250 T/h and energy efficiency of a bench scale steelmaking furnace capable of 3-6 kg/h are not in the same level of comparison. However, the energy consumption measured from the bench MW/EAF steelmaking tests is still useful information to indicate the energy efficiency to a certain degree. Energy consumption of future steel production using MW/EAF technology could be estimated with reference of the information. 
We have conducted many steelmaking tests using the bench scale MW/EAF furnace. Steelmaking times were very different from test to test. It depended strongly on the operation skill of the furnace operator and the pellet composition. The best operation starts arcing immediately after the iron ore is reduced to the degree necessary for good electric conduction and has no bridging problems, otherwise a lengthy arcing time is required. A great amount of fine lime powder blended into the iron ore pellets is detrimental to early good conductivity of the reduced iron ore mass. Less lime addition is preferred to shorten steelmaking time.

Most steelmaking time varied from 15 to 30 minutes. The shortest test was 12 minutes. The experimental energy consumption was determined based on this best operation. Table 5.4 summarizes the experimental result, composition of pellet sample, and coal. Based on these data, the energy consumption of bench MW/EAF steelmaking is given in Table 5.5.

\begin{tabular}{|c|c|c|c|c|c|c|c|c|c|c|c|c|}
\hline \multicolumn{1}{|c|}{ Table 5.4. Best Test experimental result and compositions of pellet sample \& raw materials } \\
\hline \multicolumn{10}{|c|}{ Experimental result } & \multicolumn{7}{c|}{ Pellet sample } & $\begin{array}{c}\text { Magnetite } \\
\text { ore }\end{array}$ & \multicolumn{3}{c|}{ Coal } \\
\hline $\begin{array}{c}\mathrm{t}_{\mathrm{M}} \\
(\mathrm{s})\end{array}$ & $\begin{array}{c}\mathrm{t}_{\mathrm{EA}} \\
(\mathrm{s})\end{array}$ & $\begin{array}{c}\mathrm{I} \\
(\mathrm{A})\end{array}$ & $\begin{array}{c}\mathrm{V} \\
(\mathrm{V})\end{array}$ & $\begin{array}{c}\mathrm{P}_{\mathrm{M}} \\
(\mathrm{W})\end{array}$ & $\begin{array}{c}\mathrm{Y} \\
(\%)\end{array}$ & $\mathrm{C}_{\mathrm{cm}}$ & $\mathrm{C}_{\mathrm{lm}}$ & $\mathrm{C}_{\mathrm{bm}}$ & $\begin{array}{c}\mathrm{W} \\
(\mathrm{g})\end{array}$ & $\begin{array}{c}\mathrm{C}_{\mathrm{m}} \\
(\%)\end{array}$ & $\begin{array}{c}\mathrm{C}_{\mathrm{v}} \\
(\%)\end{array}$ & $\begin{array}{c}\mathrm{C}_{\mathrm{c}} \\
(\%)\end{array}$ \\
\hline 420 & 300 & 150 & 50 & 6000 & 94.8 & 15 & 5 & 5 & 800 & 81.2 & 18.78 & 71.92 \\
\hline
\end{tabular}

\begin{tabular}{|l|c|c|c|c|c|}
\hline \multicolumn{7}{|c|}{ Table 5.5. Specific energy consumption } \\
\hline \multicolumn{2}{|c|}{} & Microwave & Electric Arc & Coal & Total \\
\hline \multirow{2}{*}{$\begin{array}{l}\text { Specific } \\
\text { energy }\end{array}$} & $(\mathrm{kJ} / \mathrm{kg})$ & 7063 & 6307 & 2521 & 15892 \\
\cline { 2 - 6 } & $\mathrm{Btu} / \mathrm{kg}$ & 6679 & 5963 & 2384 & 15027 \\
\cline { 2 - 6 } & $\mathrm{kWh} / \mathrm{kg}$ & 1.96 & 1.75 & 0.7 & 4.41 \\
\hline \multicolumn{2}{|l}{ Percentage $(\%)$} & 44.45 & 39.69 & 15.87 & 100 \\
\hline
\end{tabular}

Energy consumption of a modern EAF furnace is $1440-1,800 \mathrm{KJ} / \mathrm{kg}$, which includes the energy required to heat scrap steel from room temperature to $850^{\circ} \mathrm{C}$. Our bench scale $\mathrm{MW} / \mathrm{EAF}$ furnace started arcing after the DRI had already reached $850^{\circ} \mathrm{C}$. It consumed 6,307 KJ/kg energy, 3.5-4.4 times higher than that of a modern EAF furnace. The large difference of arcing energy between an industrial furnace and a bench scale furnace tells that the experimental energy data derived from the operation of the bench scale furnace cannot be compared directly with the energy data of an industrial furnace.

However, it is crucial to know the energy efficiency of future industrial furnaces of MW/EAF steelmaking at the beginning of extensive research. Therefore, we will make some estimations. We assume 1) energy efficiency of future industrial furnace for MW iron ore reduction will be 
$20 \%$ higher than that of the present bench scale furnace; 2) the same amount of coal will be used; and 3) the energy consumption of acing hot DRI by future MW/EAF will be the energy consumption of the present industrial EAF minus the energy of heating scrap steel from room temperature to $850^{\circ} \mathrm{C}$. The theoretical energy of heating scrap steel from room temperature to $850^{\circ} \mathrm{C}$ is $373 \mathrm{KJ} / \mathrm{kg}$ (specific heat of steel equal to $0.452 \mathrm{KJ} / \mathrm{kg}^{\circ} \mathrm{C}$ ). Assuming $65 \%$ arcing efficiency of present EAF, the consumed energy will be $574 \mathrm{KJ} / \mathrm{kg}$.

The total energy consumption of industrial MW/EAF furnace will be $5,650 \mathrm{KJ} / \mathrm{kg}+2,521 \mathrm{KJ} / \mathrm{kg}$ $+1,440 \mathrm{KJ} / \mathrm{kg}-574 \mathrm{KJ} / \mathrm{kg}=9,037 \mathrm{KJ} / \mathrm{kg}$ to $5,650 \mathrm{KJ} / \mathrm{kg}+2,521 \mathrm{KJ} / \mathrm{kg}+1,800 \mathrm{KJ} / \mathrm{kg}-574$ $\mathrm{KJ} / \mathrm{kg}=\mathbf{9 , 3 9 7} \mathrm{KJ} / \mathbf{k g}$.

The MW/EAF steelmaking technology offers a possibility of utilizing a sealed MW DRI reducing furnace as shown in Figure 2.15. The furnace is also a gaseous fuel generator during the MW DRI production. The energy in the off-gas $(246 \mathrm{KJ} / \mathrm{kg}$ as given in Table 5.3 and much more if more than theoretical minimum amount of coal is used) could be effectively utilized. The $\mathrm{MW} / \mathrm{EAF}$ steelmaking energy could be further reduced.

\subsection{Energy Consumption Comparison}

Currently, two steelmaking routes are used by the steel industry. One is based on blast BF/BOF; the other is based on EAF. The charge of EAF is scrap steel and DRI. To make a comparison based on equal foot, the EAF steelmaking in this discussion uses DRI as the charge for total energy consumption calculation.

Table 5.6 shows the energy consumption of conventional steelmaking and the new direct steelmaking. All energy consumptions are collected from good practice. The energy consumption associated with manufacture of electrode, refractory, etc. are excluded, and energy credits in off gases are also excluded.

\begin{tabular}{|c|c|c|c|c|c|}
\hline \multicolumn{6}{|c|}{ Table 5.6. Specific energy consumption comparisons } \\
\hline \multicolumn{4}{|c|}{ Conventional steelmaking } & \multicolumn{2}{|c|}{ Direct steelmaking } \\
\hline Process & $\begin{array}{c}\text { Specific energy } \\
(\mathrm{MJ} / \mathrm{kg})\end{array}$ & Process & $\begin{array}{l}\text { Specific energy } \\
(\mathrm{MJ} / \mathrm{kg})\end{array}$ & Process & $\begin{array}{c}\text { Specific energy } \\
(\mathrm{MJ} / \mathrm{kg})\end{array}$ \\
\hline $\begin{array}{l}\text { Iron ore } \\
\text { processing }\end{array}$ & 2.5 & \begin{tabular}{|l|} 
Iron ore \\
processing
\end{tabular} & 2.5 & \begin{tabular}{|l|} 
Iron ore \\
processing
\end{tabular} & 2.5 \\
\hline Shipping & 1.1 & Shipping & 0.94 & Shipping & 0.94 \\
\hline Pelletizing & 1.9 & Pelletizing & 1.9 & Pelletizing & 1.9 \\
\hline $\begin{array}{l}\text { Limestone } \\
\text { calcination }\end{array}$ & 1.1 & $\begin{array}{l}\text { Direct } \\
\text { reduction* }\end{array}$ & 14.9 & $\begin{array}{l}\text { Limestone } \\
\text { calcination }\end{array}$ & 0.5 \\
\hline Sintering & 1.7 & EAF & 2.77 & MW/EAF & $9.04-9.40$ \\
\hline Coke making & 1.67 & & & & \\
\hline BF & 17.0 & & & & \\
\hline BOF & 0.95 & & & & \\
\hline Total & 27.92 & Total & 23.01 & Total & $14.88-15.24$ \\
\hline
\end{tabular}


The difference of shipping energy consumption comes from shipping less weight due to the elimination of coke shipping and less use of limestone. Sulfur in liquid iron produced by BF comes from the direct contact of liquid iron with coke. No coke in the new process reduces the need for limestone to remove sulfur. The energy difference of limestone calcination comes from limestone weight reduction in the new process.

Table 5.6 shows that conventional steelmaking requires about $27.92 \mathrm{MJ}$ or $23.01 \mathrm{MJ}$ to produce one kilogram of steel, while the new steelmaking requires about $15 \mathrm{MJ}$. It means a $46 \%$ or $35 \%$ energy saving in comparison with the two conventional steelmaking routes. The comparison indicates that MW/EAF steelmaking is more energy efficient than conventional steelmaking.

Replacing the combination of BF and BOF or the combination of Midrex and EAF with more efficient MW/EAF contributes the major proportion of energy saving for the new steelmaking process. Eliminating coke making and pellet sintering and reducing shipping weight and limestone requirements further increases the energy savings for the new process.

The energy consumption of MW/EAF steelmaking is 9.04-9.40 MJ/kg and its minimum theoretical energy consumption is $5.21 \mathrm{MJ} / \mathrm{kg}$. The minimum theoretical energy consumption of $\mathrm{BF} / \mathrm{BOF}$ is about $9.9 \mathrm{MJ} / \mathrm{kg}$. The minimum theoretical energy consumption of Midrex and EAF is about $9.2 \mathrm{MJ} / \mathrm{kg}$. These theoretical data indicates MW/EAF process is inherently more energy efficient and it mainly results from eliminating reduction of silica and manganese oxide in the MW/EAF process.

\subsection{Summary}

$\mathrm{MW} / \mathrm{EAF}$ process is a more energy efficient steelmaking process than conventional steelmaking processes. It could achieve an energy saving of $46 \%$ over $\mathrm{BF} / \mathrm{EAF}$ steelmaking and an energy saving of $35 \%$ over Midrex and EAF steelmaking.

The minimum theoretical energy consumption using low volatile coal is $5.21 \mathrm{MJ} / \mathrm{kg}$ in $\mathrm{MW} / \mathrm{EAF}$ process, about $45 \%$ is consumed by steel and $22 \%$ to $28 \%$ is consumed by water vapor and offgas. Utilization of energy in offgas will play an important role to further increase energy efficiency. It is particularly important when high volatile coal is used as the reductant. MW/EAF steelmaking offers a good opportunity for controlling offgas composition. A combination of MW DRI furnace and gaseous fuel generator could be designed, fabricated and used.

The theoretical energy consumption of MW/EAF process varies with the volatile content of coal and the amount of coal in pellets. Pellets with high volatile coal or abundant amounts of coal have higher theoretical energy consumption. 


\section{Task 5b - Environmental Assessment}

The iron and steel industry is crucial to the U.S. economy, however, it is associated with environmental issues. Current operations have raised significant concerns because of their 1) link to high energy consumption; 2) discharge of pollutants; 3) inherent demand for coke production; and 4) enormous amount of water consumption. The steel industry has invested over $\$ 6$ billion over the last 25 years for environmental control, which represents approximately $15 \%$ of the total operating cost of the industry.

Although great efforts had been made, many steel operations were still shut down or moved to other countries due to difficulties or high cost of compliance with stringent environmental regulations. As the Steel Industry Technology Roadmap points out, the industry needs pollution prevention technologies to reduce costs, improve profitability, and facilitate compliance with changing Federal regulations. The steel industry's environmental goal is to avoid pollution rather than control and treat it. MW/EAF steelmaking technology will eliminate the operations of coking, sintering, BF steelmaking and BOF steelmaking, thus eliminating their associated environmental problems.

This environmental assessment consists of two studies: offgas emission analysis and estimation of environmental benefits. The objective of the offgas emission analysis is to identify potential emission problems, if any, associated with the new process. The estimation of environmental benefits is to calculate the possible benefits, assuming the technology is fully executed.

\subsection{Offgas Emission Analysis}

In MW/EAF process, offgas emission would contain $\mathrm{CO}_{2}, \mathrm{CO}, \mathrm{SO}_{2}, \mathrm{NO}_{\mathrm{x}}$ and VOCs, because these pollutants are combustion-related with coal as the reductant. $\mathrm{NO}_{\mathrm{x}}$ is formed when nitrogen reacts with oxygen at appropriate conditions such as high temperature and arcing. $\mathrm{SO}_{2}$ is produced from burning some of the sulfur in coal. Coal and iron ore contain organic compounds to some extent. When there is insufficient oxygen available for combustion, some of these organic compounds get into the offgas system, therefore VOCs can be burned off in the furnace by after-burning.

Assuming the volatile materials and fixed carbon in coal are completely oxidized, the amount of carbon dioxide emitted from a pellet sample with specific composition can be calculated by the following equation:

$n_{\mathrm{CO}_{2}}=\frac{W}{\left(100+C_{c m}+C_{l m}+C_{b m}\right)}\left(\frac{C_{c m} C_{v}}{16}+\frac{C_{c m} C_{c}}{12}+\frac{C_{b m}}{14}\right)$

Where $C_{b m}$ is the weight ratio of binder to magnetite ore with weight 100 in pellet sample; $C_{l m}$ is the weight ratio of lime to magnetite ore with weight 100 in pellet sample; $C_{c m}$ is the weight ratio of coal to magnetite ore with weight 100 in pellet sample, $\mathrm{C}_{\mathrm{v}}$ is the volatile materials content of coal, and $\mathrm{C}_{\mathrm{c}}$ is the fixed carbon content of coal.

Using the measured offgas composition and the total amount of carbon dioxide emitted from pellet sample, the amount of other gases in offgas can be calculated. If the concentration of gas 
such as $\mathrm{SO}_{2}, \mathrm{NO}_{\mathrm{x}}$ and VOCs is too low to be detected by equipment, the possible maximum amount can be estimated by using the minimum measurable possible with the equipment.

\section{Experimental Method}

\section{$\underline{\text { Apparatus }}$}

An offgas analysis system was set up to carry out this study. The constituent parts in the offgas analysis system are shown in Figure 5.2. Figure 5.3 is the schematic diagram of the offgas analysis system. In order to protect the gas analyzer, ceramic fibers were used as a filter to separate fine particulate from the gas emission. A vacuum pump was used to purge offgas flow. The main equipment and analysis instruments are listed as follows.

(1) Bench-scale microwave assisted electric arc furnace (MW/EAF) system

- Used for conducting direct steelmaking

(2) Quadrupole gas analyzer

- Used for analyzing offgas composition

- Manufacturer: AMETEK Thermox Instruments Division, USA

- Model: DYCOR ${ }^{\mathrm{TM}}$ M100/M200

- Mass Range: 1 - 200

- Maximum Temperature: $350^{\circ} \mathrm{C}$

- Minimum Measurable Current: 1.0E -13 amps

- Maximum Measurable Current: 1.0E -5 amps

- Accuracy: $1 \%$ of signal from $1.0 \mathrm{E}-13$ to $1.0 \mathrm{E}-8$ amps

(3) Hydrogen sulfide monitor

- Used for detecting hydrogen sulfide concentration of offgas

- Manufacturer: GC Industries Incorporation, USA

- Model: GC-701

- Measurable Range: 0 - 100 ppm

- Accuracy: \pm 3 ppm

(4) Carbon monoxide monitor

- Used for detecting carbon monoxide concentration of offgas

- Manufacturer: GC Industries Incorporation, USA

- Model: GC-401

- Measurable Range: 0 - 100 ppm

- Accuracy: \pm 10 ppm 


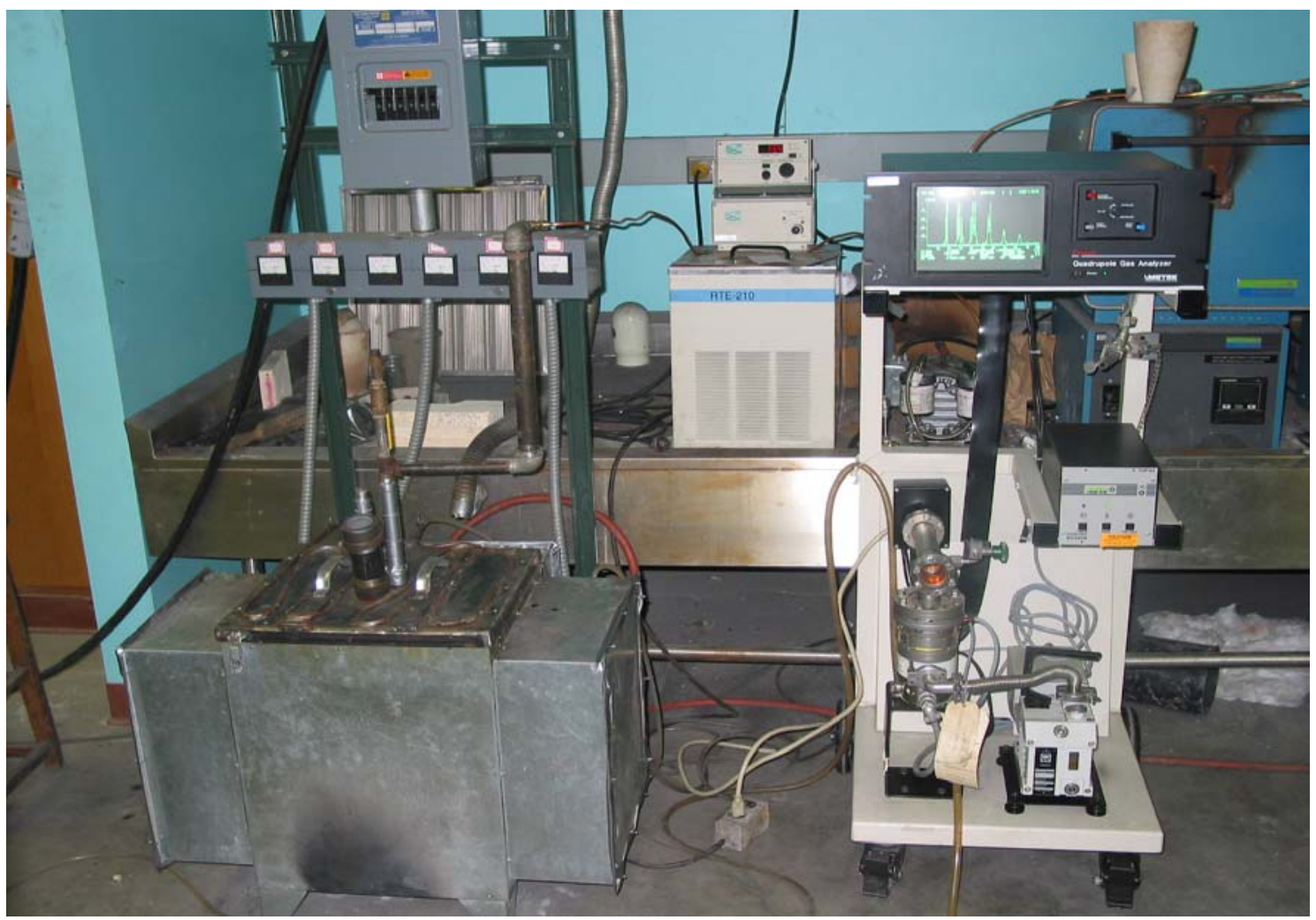

Figure 5.2 Photograph of constituent parts in offgas analysis system

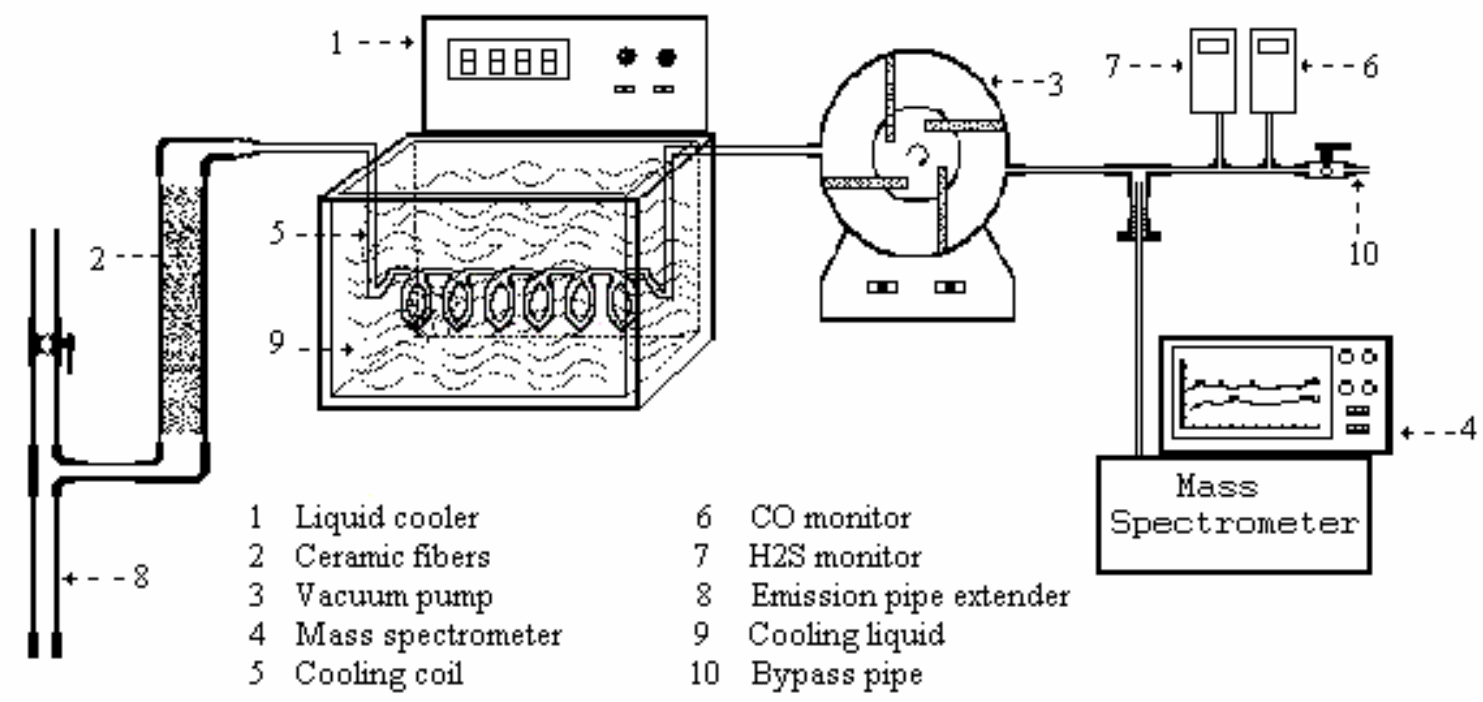

Figure 5.3. Schematic diagram of offgas analysis system 


\section{Materials and Procedures}

Offgases from nine pellet samples with different composition were analyzed. The compositions of the pellet samples were listed in Table 1.7 and Table 1.8. The composition of raw materials was listed in Table 1.9.

For the purpose of comparison, offgas emitted from coal heated through microwave heating was also analyzed. The coal composition was listed in Table 1.10.

Direct steelmaking was carried out in the MW/EAF system, and the procedure for reduction of pellets is the same as Task 1.2. The parameters of these direct steelmaking experiments were summarized in Appendix B. Offgas was monitored in-situ from the beginning of MW/EAF process. The frequency of data collection is roughly one measurement every two minutes.

\section{Results and Discussion}

The compositions of offgases emitted from nine pellet samples and coal are listed in Appendix C. The major components of offgas are nitrogen, oxygen, argon, carbon dioxide, sulfur oxide, water vapor and carbon monoxide. $\mathrm{NO}_{\mathrm{x}}$ and VOCs could not be detected by using the gas analyzer due to their lower concentrations.

The compositions of offgases emitted from pellets and coal during microwave heating illustrate the following: Carbon dioxide is generated soon after microwave heating is started. There is carbon monoxide in offgas during the early stage of microwave heating. The maximum concentration of carbon monoxide is $423 \mathrm{ppm}$. The carbon dioxide and water vapor content of offgas usually increases and the oxygen content of offgas decreases while microwave heating continues.

The composition of offgas emitted from the pellet sample during electric arc heating varies slightly with heating time. Because the temperature during electric arc heating varies slightly, the variation of offgas composition with time in MW/EAF process indicates that temperature has a determining influence on offgas composition.

Table 5.7 displays the average offgas composition over the entire process and its two stages respectively. The data about the content of carbon dioxide and sulfur dioxide indicates the following: The average carbon dioxide content of offgas emitted during electric arc heating is generally greater than during microwave heating. However, the average sulfur dioxide content of offgas emitted during electric arc heating is generally smaller than during microwave heating.

Table 5.8 illustrates that the average composition of offgas emitted from pellet sample during microwave heating is greatly different from the average composition of offgas emitted from microwave heated coal. The carbon dioxide content of the pellet sample's offgas is nearly 2.5 to 5 times that of coal's offgas, however the sulfur dioxide content of coal's offgas is nearly 3 to 11 times that of the pellet sample's offgas. 


\begin{tabular}{|c|c|c|c|c|c|c|c|c|c|c|c|c|c|c|c|c|c|c|c|c|c|}
\hline & \multicolumn{3}{|c|}{$\begin{array}{l}\mathrm{CO}_{2} \\
(\%)\end{array}$} & \multicolumn{3}{|c|}{$\begin{array}{c}\mathbf{N}_{2} \\
(\%)\end{array}$} & \multicolumn{3}{|c|}{$\begin{array}{c}\mathbf{O}_{2} \\
(\%)\end{array}$} & \multicolumn{3}{|c|}{$\begin{array}{l}\mathrm{SO}_{2} \\
(\%)\end{array}$} & \multicolumn{3}{|c|}{$\begin{array}{c}\mathrm{H}_{2} \mathbf{O} \\
(\%)\end{array}$} & \multicolumn{3}{|c|}{$\begin{array}{c}\text { Ar } \\
\text { (\%) }\end{array}$} & \multicolumn{3}{|c|}{$\begin{array}{l}\text { CO } \\
\text { (\%) }\end{array}$} \\
\hline & MV & EA & All & MV & EA & All & MV & EA & All & MV & EA & All & MV & EA & All & MV & EA & All & MV & EA & All \\
\hline$\# 1$ & 5.63 & 9.40 & 6.26 & 75.30 & 74.72 & 75.20 & 16.06 & 13.48 & 15.63 & 0.032 & 0.030 & 0.031 & 1.80 & 2.37 & 1.89 & 1.18 & 1.28 & 1.20 & 0.0048 & 0 & 0.0040 \\
\hline$\# 2$ & 5.19 & 8.80 & 5.79 & 78.44 & 78.92 & 78.52 & 13.57 & 9.29 & 12.86 & 0.024 & 0.007 & 0.021 & 1.61 & 1.80 & 1.64 & 1.17 & 1.19 & 1.17 & 0.0064 & 0 & 0.0053 \\
\hline$\# 3$ & 6.94 & 14.9 & 8.26 & 79.88 & 76.50 & 79.57 & 11.44 & 8.22 & 10.90 & 0.049 & 0.008 & 0.042 & 1.40 & 1.01 & 1.40 & ND & ND & - & ND & ND & - \\
\hline$\# 4$ & 7.40 & 9.88 & 7.85 & 77.48 & 78.35 & 77.64 & 14.50 & 9.88 & 13.66 & 0.024 & 0.007 & 0.021 & 0.60 & 1.85 & 0.82 & ND & ND & - & ND & ND & - \\
\hline$\# 5$ & 5.79 & 10.29 & 6.52 & 76.39 & 75.89 & 76.31 & 13.92 & 10.13 & 13.29 & 0.021 & 0.011 & 0.019 & 2.39 & 2.00 & 2.33 & 1.51 & 1.68 & 1.54 & 0.0024 & 0 & 0.0020 \\
\hline$\# 6$ & 10.01 & 9.92 & 9.99 & 75.99 & 77.15 & 76.2 & 12.30 & 10.35 & 11.95 & 0.036 & 0.006 & 0.031 & 1.69 & 2.59 & 1.85 & ND & ND & - & ND & ND & - \\
\hline$\# 7$ & 5.2 & - & - & 79.10 & - & - & 12.64 & - & - & 0.013 & - & - & 1.92 & - & - & 1.14 & - & - & 0.0045 & 0 & - \\
\hline$\# 8$ & 9.81 & 10.7 & 9.96 & 76.70 & 78.00 & 76.94 & 12.05 & 10.04 & 11.69 & 0.027 & 0.016 & 0.025 & 1.42 & 1.27 & 1.39 & ND & ND & - & ND & ND & - \\
\hline$\# 9$ & 6.68 & 10.56 & 7.33 & 77.83 & 78.87 & 78.00 & 13.94 & 8.08 & 12.97 & 0.024 & 0.024 & 0.024 & 1.53 & 2.48 & 1.69 & 1.26 & 1.23 & 1.26 & 0.0019 & 0 & 0.0016 \\
\hline Coal & 2.03 & - & - & 79.71 & - & - & 16.31 & - & - & 0.145 & - & 0.145 & 1.79 & - & - & ND & ND & - & ND & ND & - \\
\hline
\end{tabular}

MV: microwave heating, EA: electric arc heating, All: microwave and electric arc heating, ND: not detected, - : not applicable

\begin{tabular}{|c|c|c|c|c|c|}
\hline \multicolumn{5}{|c|}{ Table 5.8. Estimated intensities of combustion-related pollutants from the MW/EAF process } \\
\hline Pollutant & $\mathbf{S O}_{2}$ & $\mathbf{N O}_{\mathbf{x}}$ & $\mathbf{C O}$ & VOC $_{\mathbf{s}}$ & $\mathbf{C O}_{2}$ \\
\hline $\begin{array}{c}\text { Intensity } \\
\text { (lbs/ton of steel) }\end{array}$ & $\leq 23.15$ & $\leq 0.01$ & $\leq 1.86$ & $\leq 0.015$ & 3183 \\
\hline
\end{tabular}


Rapid microwave heating rate of magnetite and magnetite reduction reaction in pellet samples are believed to contribute to the high carbon dioxide content of pellet sample's offgas. Compared with coal, pellet samples can be rapidly heated by microwave to an elevated temperature due to its magnetite constituent. The rates of coal combustion and magnetite reduction are greatly enhanced at higher temperature; therefore more carbon dioxide is emitted. Low sulfur dioxide content of pellet sample's offgas is contributed by the lime constituent of the pellet sample. The large proportion of sulfur in the pellet sample is retained in pellet sample due to reaction with lime, so lime in the pellet sample plays a very important role on reducing sulfur dioxide emission.

Table 5.8 lists the estimated intensities of combustion-related pollutants from pellet samples in the MW/EAF process. The intensity of carbon dioxide was estimated by using equation 5-36, and pellet samples with high volatile coal whose weight ratio was 35/100. The intensities of sulfur dioxide and carbon monoxide were estimated by using the intensity of carbon dioxide and measured maximum ratios. The intensities of $\mathrm{NO}_{\mathrm{x}}$ and VOCs were estimated by using the intensity of carbon dioxide, measured carbon dioxide content of offgas, and the minimum measurable current of quadrupole gas analyzer.

Compared with the estimated intensity of combustion-related emissions from BF (blast furnace), offgas from the MW/EAF process contains less combustion-related pollutants.

The MW/EAF process can reduce carbon dioxide and sulfur dioxide emission by $34 \%$ and $26 \%$, which is due to less coal used for one ton of steel. Coke is used as the major fuel in BF and the estimated intensity of carbon dioxide for $\mathrm{BF}$ is $2000 \mathrm{lbs}$ per ton of iron. If carbon dioxide emission from coke making is taken into account, $4840 \mathrm{lbs}$ carbon dioxide is released for producing one ton of pig iron.

$\mathrm{MW} / \mathrm{EAF}$ process reduces carbon monoxide emission by $68 \%$ with respect to BF. VOCs and $\mathrm{NO}_{\mathrm{x}}$ emission from MW/EAF process are almost insignificant (only 0.01 and $0.015 \mathrm{lbs}$ per ton of steel). The low CO and VOCs emission in offgas indicate that almost all carbon monoxide and volatile organic compounds can be completely oxidized in the MW/EAF furnace before they get into the offgas system.

\section{Summary}

The major components of pellet sample's offgas are nitrogen, oxygen, argon, carbon dioxide, sulfur oxide, water vapor and carbon monoxide. The offgases emitted during the two stages of the MW/EAF process have different constituents and remarkably different composition; carbon monoxide is emitted during the early stage of microwave heating.

The average composition of offgas from the MW/EAF process by volume percent typically is $5 \%-11 \% \mathrm{CO}_{2}, 75 \%-79 \% \mathrm{~N}_{2}, 10 \%-16 \% \mathrm{O}_{2}, 0.01 \%-0.04 \% \mathrm{SO}_{2}, 1 \%-3 \%$ water vapor, $1.1 \%-1.7 \% \mathrm{Ar}$ and $0.002 \%-0.005 \% \mathrm{CO}$. The maximum concentration of carbon monoxide measured in our investigation is $423 \mathrm{ppm}(0.0423 \%)$. 
Compared with conventional steelmaking, the MW/EAF process can greatly reduce combustionrelated pollutants. With respect to $\mathrm{BF}$, the $\mathrm{MW} / \mathrm{EAF}$ process can reduce carbon monoxide and sulfur dioxide emission by $68 \%$ and $26 \%$; and VOCs and $\mathrm{NO}_{\mathrm{x}}$ emissions from the MW/EAF process are insignificant. The intensity of carbon dioxide emission from the MW/EAF process is $3183 \mathrm{lbs}$ per ton of steel. With respect to coke making and BF, carbon dioxide emission is reduced by $34 \%$ because less coal is used in the MW/EAF process.

\subsection{Estimation of Environmental Benefits}

The environmental benefits will come from the elimination of cokemaking, pellet sintering, BF ironmaking, BOF steelmaking, and significant energy savings.

Sintering: Sinter plants emit particulate and $\mathrm{CO}$, and discharge waste water. The emission rate of $\mathrm{CO}$ is about $0.47 \mathrm{lb} /$ ton of sinter and the discharge rate of waste water is about 1,500 gallons/ton of sinter. The emissions and generation of waste water are due to combustion of fuel. Waste water is generated from scrubbers used for wet air pollution control. To produce one ton of steel by BF/BOF, about 1.35 tons of sinter is required. Currently, the United States produces about 46 million tons of steel by integrated mills. It translates into emission of 29.2 million pounds of $\mathrm{CO}$ and discharge of 93,150 million gallons of waste water annually in the United States.

BF Ironmaking: To produce one ton of liquid iron, BF operations generate 100 pounds of particulate, 2.5 to 3.5 tons of furnace gas, and 6,000 gallons of waste water. The furnace gas contains up to $40 \% \mathrm{CO}$ and $6 \% \mathrm{H} 2$. Nearly all of the waste water is direct contact water used in the gas coolers and wet scrubber of furnace dust. The generation of great amounts of particulate, furnace gas and waste waters are result of coke combustion in BF. The combustion requires great amount of air blown into the furnace. The United States produces about 46 million tons of iron at present. Therefore, the BF operations generate 4,600 million pounds of particulate, 115 to 161 million tons of furnace gas and 276,000 million gallons of waste water per year.

BOF Steelmaking: To produce one ton of steel, BOF operations generate approximately $10 \mathrm{~kg}$ of CO emissions, 8 pounds of dust, 62 pounds of sludge, and 1,100 gallons of waste water. The generation of these by-products is due to the high speed and high volume oxygen blown into the BOF to react with carbon in liquid iron. The United States generated 617,000 tons of CO emission in 1992 and 270,000 tons of dust and 1.3 million tons of sludge in 1996. Currently the United States produces about 46 million tons of steel by integrated mills. It translates into production of 50,600 million gallons of waste water annually.

Cokemaking: Cokemaking produces approximately 10,800 scf of coke oven gas per ton of coal charged. About $40 \%$ of the gas is used and the rest cause air emission problems. The emissions include ammonia, benzene-soluble organics, benzene, particulates, sulfur oxides, and volatile organic compounds. Cokemaking also generates approximately 100 gallons of waste water and 25 to 35 gallons of waste ammonia liquor per ton of coke produced. The waste water contains significant amounts of oil and grease, ammonia-nitrogen, cyanide, thiocyanates, phenols, benzenes, toluene, xylene, other aromatic volatile components, and polynuclear aromatic compounds. Waste water also contains trace amounts of the toxic metals antimony, arsenic, and selenium. To produce one ton of coke, about 1.4 tons of metallurgical coal is used. The United 
States consumes about 27.6 million tons of coke each year for steelmaking. It converts to annual consumption of 38.64 million tons of metallurgical coal. In summary, the United States generates approximately 250,387 million scf of air emission, 2,760 million gallons of waste water, and 690 to 966 million gallons of waste ammonia liquor.

Energy Saving: MW/EAF steelmaking technology could save about $40 \%$ energy over BF/BOF steelmaking $(40 \% \times 27.92 \mathrm{GJ} / \mathrm{t})$, which is equal $10.57 \times 10^{6} \mathrm{BTU}$ per ton of liquid steel. Since coal is the dominant energy source for the iron and steel industry, the energy savings is equivalent to about 0.32 tons of coal for each ton of liquid steel. For about 45 million tons of steel produced by BF/BOF in the United States, it means a savings of 14.4 million tons of coal per year. Coal combustion means emissions of $\mathrm{CO}_{2}, \mathrm{SO}_{2}, \mathrm{NO}_{\mathrm{x}}$, trace elements, VOCs, fine particulates, and air toxics such as PAHs, fly ash, etc. The saving of 14.4 million tons of coal combustion will prevent the following emissions:

- 35 million tons of $\mathrm{CO}_{2}$ (use $1.2 \mathrm{lb} \mathrm{CO}_{2} / \mathrm{MBTU}$ )

- 87 thousand tons of $\mathrm{SO}_{2}$ (use $0.3 \%$ sulfur) and 0.48 million tons of lime for scrubbing (for 2\% sulfur in Midwest coal)

- 35 tons of mercury emissions ( 3 ppm Hg/ton coal)

- 1.5 million tons of fly ash

- Other emissions including $\mathrm{NO}_{\mathrm{x}}$ and air toxins.

Slag Reduction: In conventional steelmaking, iron ore concentrate must be pelletized with bentonite as the binder and lime as a desulfurizer and fluxing agent before being charged into the blast furnace. The bentonite and limestone consume energy during iron and steelmaking, but also yield large tonnages of slag that must be disposed of. Since the new technology would use iron ore concentrate directly without additions of bentonite and, since sulfur contamination is present from western coal, only a small amount of lime is needed as a fluxing agent. Thus, slag generation is reduced and mining of bentonite and limestone can be reduced, respectively. 


\section{Task 6: Marketing and Economic Assessments}

This chapter consists of two sections: marketing assessment and economic evaluation of MW assisted steelmaking process.

\subsection{Marketing Assessment}

This section discusses the marketing and economics of current steel and iron-making technologies to provide the basis for showing how the implementation of the microwave steelmaking technology will improve the competitiveness of the US steel industry. The section is divided into three parts. The first part looks at the current technologies used in the steel and ironmaking industries. The second part looks at the trends occurring in steel production and consumption for the world and the USA. From this information, the possible ways the microwave steel-making technology can be used to improve the competitiveness of US steel production are discussed. The third part looks at the cost of using the microwave steel-making technology and how it compares with existing technologies.

\section{Current steel-making and iron-making technologies}

Today, two technologies dominant the commercial production of steel worldwide: the Basic Oxygen Furnace (BOF) and the Electric Arc Furnace (EAF). The BOF technology accounts for about $60 \%$ of steel output in the world and $50 \%$ for the USA. This process is an indirect method that first produces iron (carbon content greater than 2.08\%) and then converts it to steel (carbon content less than 2.08\%). To make iron and steel, iron ore is ground to 500 mesh (22 mm) to liberate iron oxides from other minerals. The iron in the ground materials is concentrated by magnetic separation and froth flotation. The fine oxide particles cannot be fed to a smelter directly so they are pelletized with limestone and bentonite, sintered to make them strong, and then charged along with coke into the furnace. The raw materials are then subjected to a blast of very hot air. In the furnace, the iron oxide is reduced and melted and gains carbon and sulfur from the coke during melting. The carbon content in the iron must be lowered to make steel. This is commonly done in a Basic Oxygen Furnace, where pure oxygen is blown into the liquid iron at an ultra-sonic velocity to oxidize the excess carbon to form $\mathrm{CO}$ and $\mathrm{CO}_{2}$. The gases are released to the atmosphere.

The EAF is the second major process for producing steel and accounts for about $40 \%$ of steel output in the world and 50\% in the USA. Traditionally, $100 \%$ cold steel scrap is charged into an electric arc furnace and melted by intensive electric current between graphite electrodes and the electrically conductive raw materials. As an alternative to steel scrap, Directly Reduced Iron (DRI) has been developed and used to replace a percentage of steel scrap. Steel-making by the EAF process depends on the availability of scrap and the use of DRI, or pig iron produced by blast furnaces. DRI and pig iron usually contain fewer impurities than steel scrap and are preferred for the production of higher-grade steels. It is predicted that DRI will account for $25 \%$ to $50 \%$ of the EAF raw material charges by 2015.

Iron-making constitutes the process whereby iron oxides are chemically reduced to a metallic state by a reductant. The main iron-making technologies currently employed are the blast furnace technology (BF) and DRI. The BF technology as we recognize it today has been employed for 
approximately 150 years. The blast furnace is a vertical shaft furnace where raw materials are charged at the top and hot metallic iron and slag are topped from the bottom of the furnace. In the BF, iron ore, coke, and limestone are charged in layers at the top of the furnace. The charge direction is countercurrent to the gas flow in the stack. As the burden descends it comes in contact with hot gases that are rising in the stack. In the process, the coke is reheated as it descends so that when it arrives at the lower portion of the furnace, its contact with air from the hot blast makes it burn with intensity. With the high temperatures that exist, carbon dioxide is not stable because of the excess carbon present. The carbon dioxide reacts with the excess carbon to form carbon monoxide, the chemical reductant of the iron making process. As the iron ore and impurities work their way down through the stack they melt, forming liquid hot metal and slag. The liquid hot metal and slag are tapped from different locations periodically. The slag, the waste product of the process, is ladle transported, cooled, sized, and used as construction material. The hot metal proceeds by iron ladle to the refining process (primarily BOF) or is sent to the pig machine to be cast into solid ingots. Hot metal quality is typically $95 \% \mathrm{Fe}, 3.5 \% \mathrm{C}$, and $1.5 \%$ other impurities.

In the subsequent refining operations, the hot metal is transferred to the BOF where it reacts with pure oxygen to burn out excess carbon and silicon. The liquid steel is then cast into billets or slabs for further processing by hot rolling and cold rolling. Steel made from ore in this manner is typically used for the highest quality applications. Approximately 45 million tons of steel per year are produced in the USA using this method.

On a worldwide basis DRI is a small but quickly growing source of metallic iron. DRI is a process to produce sponge iron or iron powder from iron ore using a gaseous or coal based reductant without a smelting process. Establishment of DRI plants can be traced to the early part of this century. Extensive direct reduction research however did not start until 1950s and several processes have been developed since such as Midrex, HyL, Fior, FINMET, Höganäs, KruppRenn, Iron Carbide, Circored, FASTMET, INMETCO, SL/RN, and Cicofer. Although a great deal of effort has been made, commercialization of these processes is very limited. DRI accounts for less than $10 \%$ of the input used in world steel production. This is up from the less than $3 \%$ in the 1980s. Approximately 5 million tons of DRI are imported into the USA per year, mainly to supplement scrap in EAF operations and to supplement iron units in the blast furnace.

The remaining iron-making technologies can be categorized by the type of reductant used natural gas or coal. The resultant products are DRI, sponge iron, and, in one case, hot metal. In the USA, these types of processes account for less than one million tons per year of steel production. Where natural gas is used as the reductant, the gas is either used directly or cracked in a reformer chamber into hydrogen and carbon monoxide. Worldwide, gas based processes account for about 50 million tons per year of iron production, out of a world steel market of over 800 million tons per year. Gas is the reductant of choice where inexpensive natural gas is readily available, including countries like Malaysia, Bahrain, Mexico, Venezuela, Saudi Arabia, and Trinidad-Tobago. Gas based processes that are in commercial use or are being looked at for commercial development are the Midrex Shaft Furnace, HYL process, and various fluidized bed processes.

The Midrex Shaft Furnace is the most widely used process in the world for DRI production, generating 35 million tons per year of metallic iron. The feed to the process is fired pellets or lump ore and natural gas. A vertical shaft furnace is utilized, with the ore traveling down the 
stack countercurrent to the reducing gas. Natural gas goes through a reformer chamber where it is catalytically cracked into hydrogen and carbon monoxide prior to entering the furnace. The off gas is recovered at the top of the furnace, used to cool the DRI, and then recycled to the reformer with additional natural gas. The product is hot sponge iron that is directly charged into an EAF or briquetted and cooled for shipping.

The original HYL process was a batch version of the Midrex shaft furnace, where multiple shafts are charged with ore, reduced, and discharged. The process has since been modified into a continuous process similar to the Midrex Process. HYL plants are the second largest producer of DRI in the world, owned by Hylsa Steel of Mexico.

There are three commercialized fluidized bed processors; the Fimet process in Venezuela, the Iron Carbide, and Cliffs-Lurgi plants in Trinidad. In these processes, closely sized iron ore fines are suspended in a fluidized bed of reducing gases at high temperature. The processes produce grains of reduced iron at high temperature. In the Fimet and Cliffs-Lurgi processes, these grains are briquetted for shipment. In the Iron Carbide process, excess carbon is absorbed from the gas to produce iron carbide. These processes have the advantage of being able to use relatively coarse fine ore. The Fimet process is commercially used in Venezuela. The Cliffs-Lurgi plant in Trinidad is currently shut down. The Iron Carbide plant, built by Nucor, has been permanently shut down and is not considered a success. Feed for these plants is not readily available in the Great Lakes region, with the most likely source being crushed and sized pellets.

Coal is the reductant for most of the iron produced from ore in the world, whether as coal itself or made into coke as used in the blast furnace. Because of the significant tonnage of hot metal produced, the blast furnace was discussed previously, but is fundamentally part of this group of processes. The other coal based ironmaking processes that are in commercial use or being developed for commercial use are the Corex process, various rotary hearth processes, Hismelt and Ausmelt processes, and the ACCAR and Grate Car processes.

The Corex process has been commercialized in South Africa and Korea, and is designed as an alternative to blast furnace production of hot metal, which does not require metallurgical coke. It is basically a two-stage process with a direct reduction shaft furnace mounted on top of a high temperature oxygen blown melting furnace. The feed to the process is either pellets or lump ore and coal that is fed into the melting furnace with oxygen. The combustion of the coal in the lower melting furnace provides heat for melting and reduction furnaces along with an ascending off gas composed of various hydrocarbons and carbon monoxide working its way through the bed of the reducing furnace section. Iron and slag are periodically tapped from the furnace and the iron produced has essentially the same quality as hot metal from a blast furnace. This is a high-energy consumption process, at about 16 million BTU per ton of hot metal after credit for off gas energy use.

Several rotary hearth ironmaking processes have been proposed over the years, starting with the Surface Combustion Heat Fast process of the early 1960's. In the typical rotary hearth process, a layer of green balls, dried green balls, or pellets made of fine iron ore concentrate, pulverized coal, and a binder are placed in a thin layer on the surface of an annular rotary hearth. As the hearth revolves through its cycle, the feed is heated by radiant heat from the hood over the hearth and supplemental burners in the hood, reducing the carbon in the coal to carbon monoxide for reduction of the iron oxides. The resulting product is sponge iron in the form of pellet or 
briquette or nodules of pig iron. The sponge iron can either be compressed and cooled or charged directly into an EAF or BOF. In the sponge iron processes, a relatively impure iron product is produced because any slag in the ore or coal and most of the sulfur in both stay with the iron for later removal.

A typical sponge iron product would be approximately $94 \%$ metallic iron and 6\% carbon-slag. It should be noted that a sponge iron product carries roughly a $10 \%$ penalty in value due to impurities. Commercial names for sponge iron processes include Inmetco/Demag, Fastmet/Midrex, Dryiron/Maumec, Iron Dynamics and others. Commercial plants have been installed for iron ore in Indiana, and for steel mill waste recycling in Pennsylvania, Michigan, Alabama, and Japan. In addition, two new processes under development independently by Kobe Steel and Kawasaki Steel use the rotary hearth to produce drops or nuggets of pig iron. The major difference is that the rotary hearth is run at a high temperature and the sponge iron pellets or briquettes melt to droplets of molten pig iron, with slag residing on the surface of the droplet, which can be easily separated by mechanical means. Nugget composition is similar to pig iron. These processes have only been run in pilot plants in Japan. A pilot plant is being assembled to test the Kobe process in Minnesota and a 50,000 tons per year (1/20 scale) demonstration plant for the Kobe process is under development for Cleveland Cliffs' Northshore site in Minnesota.

The Hismelt and Ausmelt processes are similar independently developed processes from Australia. Hismelt has been successfully demonstrated and Ausmelt is currently starting up a demonstration plant. Both processes start with a heel of molten metal in the bottom of the vessel similar to an empty blast furnace. A mixture of fine iron ore, pulverized coal, pulverized lime, preheated air and supplemental oxygen are blown at the surface of the hot metal. This ignites the coal, producing carbon monoxide and reducing the iron ore to metallic iron. The metallic iron is similar to pig iron quality. The capacity of the process is limited by the size of the combustion ball that can be maintained in the furnace.

The only other coal based processes that have shown commercial success are the ACCAR and Grate Car processes developed by Allis Mineral Systems (now Metso Minerals). In both processes, the feed to the system is fired pellets or lump ore and lump coal with pulverized coal added to the rotary kiln, where the mixture is tumbled in a reducing atmosphere. This process produces a product that is less sensitive to impurities in the coal, but still requires high quality iron oxide feed stock. The product is sponge iron pellets or briquetted sponge iron.

\section{The Global and USA Markets}

During 1975-2002, the annual world consumption of steel has been cyclical with the consumption levels of 1975 and 2002 being similar (around 750 million metric tons). The regional consumption patterns for steel, however, have been changing over this time period. Asia is the region of strongest growth in steel consumption-largely driven by China as the largest consumer of steel in the world. The other major consuming regions are the European Union (EU) and North American Free Trade Agreement (NAFTA). Within the EU, growth in consumption is being driven by Spain, Italy, and the smaller consuming nations. The larger consuming nations of the EU-France, Germany, and UK-have had a flat consumption trend. The USA is the dominant consumer in NAFTA, and its steel consumption has been increasing at an average annual rate of nearly 1\% during1975-2002. Much of the growth in consumption 
occurred in the mid-to-late 1990s. Annual steel consumption is very cyclical and varied from 70 to 120 million metric tons during 1975-2002. Annual steel consumption in 2002 was a relatively high 107 million metric tons. The US market for steel is one of the largest in the world and is not fragmented into smaller national segments like in the EU and other parts of the world that must be added together to equal it. Alone, the USA accounted for nearly $15 \%$ of total annual world steel consumption. This is up from $11 \%$ in 1975. The major buyers of steel in the USA are warehouses and steel service centers, automobile industry, construction, and cans and containers.

US steel consumption can be largely explained with its level of industrial production and the changing nature of the US economy. An ordinary least-squares model using annual data for 1975-2002 with the explanatory variables of indexed industrial production and a yearly time variable explains about $80 \%$ of the observed annual variation in consumption. The variables are highly significant: the Indexed industrial Production variable's t-statistic is 6.9 and the t-statistic for the time variable is -5.0 (A t-statistic value greater than 2.5 is significantly different from zero at the 99 percent level of confidence.). There is a small but significant trend over time for the USA of less steel being consumed each year with all else being constant. The annual per capita consumption of steel in the USA (around 376 kilos in 2001) has remained fairly constant since 1975 despite the significant economic growth that has occurred. A similar trend can be observed for the wealthiest countries of the EU and Japan.

One theory used to explain this observed behavior is the Intensity-of-Use Hypothesis. It is argued that wealthy nations will emphasize service goods over manufactured goods over time. One implication is that service goods require less material like steel over time as compared with manufactured goods. Another implication is that countries still in the process of becoming wealthy (like China and Spain) should show increasing consumption of materials like steel. The steel industry statistics do not contradict this view. However, the large level of consumption by the wealthiest nations relative to other nations will maintain their key importance to the market trends for the foreseeable future. The key exception is China with its already large level of steel consumption despite its lower average income level.

Steel production occurs throughout the world, but it is centered in EU, NAFTA, and East Asia. Annual steel production in 2002 was 870 million metric tons. This compares to 644 million metric tons in 1975. World output tends to be less cyclical than the output from individual countries. The countries will the largest levels of steel production include China, Japan, USA, Russia, Germany, and Korea. Most of the world's production capacity is privately owned, and approximately $40 \%$ of the world's steel production is traded internationally. World steel production during 1975-2002 has been growing at an average annual rate of just under one percent. On average throughout the time period, $60 \%$ of the steel production was done with the BOF technology with the remainder being produced mainly with the EAF technology. A surplus of production capacity relative to steel consumption has led to the current market trend of producer consolidation to reduce capacity. This consolidation trend is also helping to create steel producers with global capacity (in an industry where transportation costs are important) that can offset home markets that are too small to achieve full production economies-of-scale and can match key consumers like the automobile industry that are becoming more global in their demand.

Steel is produced in the USA at 140 sites by 90 companies (the number varies from year-toyear). The focus for production is around the Midwest with Indiana, Ohio, Illinois, and 
Pennsylvania being the leading producing states. Despite a period of increased production during the 1990s, the USA has had a decline in steel production during 1975-2002 that has been offset by increased imports. This decline has been centered on the production of steel with the BOF technology. The production of steel by the EAF technology has been rising over the same time period. In 1975, the BOF technology accounted for $65 \%$ of the annual US steel production with the EAF technology accounting for another 21\%. By 2002, the USA's annual production share of steel by the BOF and EAF technologies were about equal with the EAF technology output being a little greater. US producers are less interested in global alliances than other producers because they have adequate demand at home in a market they understand well. US iron ore production is also unattractive for the globalization trend because of its geographic isolation. The large US market attracts world producers who are seeking export markets. A competitive US producers' price can have no premium beyond the $\$ 40-50 /$ ton transportation costs of the lowcost foreign producers to the US market (except under tariff protection).

\section{Use of Microwave Technology to Improve Competitiveness}

BOF production in the USA is under pressure from production both domestically and abroad. Domestically, expanding, regional EAF producers have used their relatively lower production costs to take over the lower-valued steel markets from BOF producers. In the past, EAF producers have been limited by the quality of their feed material for production to lower-valued steel markets. Current efforts to improve the quality of feed material for EAF production have placed BOF higher-valued steel markets at risk as well. Lower cost imports from foreign BOF producers have also reduced the demand for domestic BOF production. Worldwide, the market for BOF steel is characterized by overcapacity and low prices. This is a difficult situation for US producers with their high legacy costs (benefits for retired workers) and older facilities. The long period of limited profits has made BOF producers unable and unwilling to put financial resources into capital investment and innovation. Overall, R \& D spending is low at only $0.5 \%$ of sales revenues (as compared to $4 \%$ on average for manufacturing industries). The microwave technology is not a probable good fit with the BOF producers and their situation to improve competitiveness.

Today, EAF producers are the major providers of wire rods, wire, bars, and structural shapes. EAF producers are also starting to move into the production of higher-value products that have been the main business of BOF producers. EAF production success in these higher-valued steel markets depends on the availability of low electricity costs and adequate quantities of highquality scrap and other iron feed inputs. EAF producers have more of a regional focus and are less concentrated in the Midwest as compared to BOF producers. This gives the EAF producers the ability to follow their customers as they shift location and keep transportation costs down. Overall, EAF operations have lower operating and capital costs than BOF operations and, correspondingly, stronger profits. The future growth of EAF production, however, depends on moving into higher quality steels. This makes the availability and price of high quality scrap a concern.

The microwave technology is an excellent fit with the EAF producers for improving competitiveness. EAF producers are very competitive in the steel industry and are gaining production share in the USA. Their move into the higher quality steels requires the availability of affordable, higher-quality scrap or suitable alternative. The EAF operators have more ability to 
fund capital investment and innovation. The ability of the EAF operators to use an alternative iron ore feed in place of scrap would improve their flexibility and competitiveness.

Existing alternative iron production methods like DRI have not made significant gains in production share in the USA so far. The problems with the current DRI processes include low productivity, low energy efficiency and relatively high production cost. These problems are caused by the fundamental fact that gas/solid or solid/solid reduction reactions take place at relatively low temperature with the assistance of inefficient external heating. The heat transfer rate, chemical reaction rate, and mass transport rate are low in these processes. For instance, Midrex and HyL, two major DRI processes, require 5 to 6 hours for complete reduction of iron ore. In addition, these two processes require pelletized iron ore, high capital costs, and production scales of at least one million tons per year to be economical. The microwave technology provides another low cost alternative iron production option that avoids these problems. An EAF producer can use the microwave technology as a supplement to its existing operations by allowing it to use iron ore feed as well as steel scrap. The cost of the addition of the microwave technology to existing EAF operations would be lower than stand-alone facilities because of the ability to use the existing capital and workforce already in place.

Another possible role for the microwave technology is as stand-alone facilities. These facilities could play a role as regional producers in a similar manner as EAF producers. These facilities could make use of excess US iron ore production that becomes available as US BOF production continues to decline. The isolation of US iron ore production makes it unlikely for it to be used by foreign producers given the ready availability of iron ore in the world. The microwave technology can provide a competitive alternative. The microwave facilities can also be located as to take advantage of savings in transportation costs between iron ore producers and steel markets.

In a global economy that is becoming more competitive, the U.S. steel industry needs technology that is responsive to changing market demands. This proposed technology will offer the steel industry flexibility in production capabilities, providing a means to explore products with new properties to meet new demands, yet capable of maintaining current product lines without penalty of additional capital investment. This flexibility is envisioned to come from a broader spectrum of feedstocks to be used, the combination of two energy input systems, and the simplification of mill equipment layouts. As this technology develops, it will be those companies who can create new operating methods and recipes to meet new demands that will establish market share. As exhibited by the spectrum of the project's industrial participants, there is strong interest in this technology and a desire to understand how their current position in the industry will be affected. 


\subsection{Economic Evaluation}

Noramco Engineering was invited to conduct an independent economic evaluation of the MW assisted steelmaking process based on production of 500,000 tons of steel per year. Noramco Engineering Corporation is a Minnesota-based major engineering firm serving the iron and steel industry (www.noramcoeng.com). Noramco sent an engineer to Michigan Tech to observe a steelmaking demonstration and collect basic technical data. The company then developed a conceptual design of a MW assisted steelmaking system as shown in Figure 2.5. Noramco's economic evaluation was based on this design. Noramco also compared the capital and operating costs of this new technology with those of existing DRI or ironmaking processes, as shown in Table 6.1. The new microwave assisted steelmaking process compares very favorably with any of the existing processes in both capital and operating costs. The details of Noramco's economic evaluation of the MW assisted steelmaking technology are given in Appendix D, Tables D.1 and D.2.

\begin{tabular}{|c|c|c|c|c|c|}
\hline \multicolumn{6}{|c|}{ Table 6.1. Comparison of Direct Reduction Processes } \\
\hline Process & Ore Source & Reductant & \begin{tabular}{|l|} 
Capital* per \\
Annual Ton
\end{tabular} & $\begin{array}{l}\text { Cash Operating } \\
\text { Cost** per Ton }\end{array}$ & End Product \\
\hline \multicolumn{6}{|l|}{ Gas Based Processes } \\
\hline Midrex & Fired Pellet or Lump & Natural Gas & $\$ 200$ & $\$ 130$ & DRI \\
\hline HYL & Fired Pellet or Lump & Natural Gas & $\$ 200$ & $\$ 130$ & DRI \\
\hline Fluidized Bad & Screened Fines & Natural Gas & $\$ 200$ & $\$ 150$ & DRI \\
\hline \multicolumn{6}{|l|}{ Coal Based Processes } \\
\hline Blast Furnace & Pellets, Lump or Sinter & Coke & $\$ 1750$ & $\$ 120$ & Liquid Iron \\
\hline Corex & Pellets, Lump & Coal & $\$ 400$ & $\$ 140$ & Liquid Iron \\
\hline Rotary Hearth & Green Balls & Pulv. Coal & $\$ 270$ & $\$ 90$ & DRI \\
\hline Rotary Hearth Nuggets & Green Balls & Pulv. Coal & $\$ 300$ & $\$ 100$ & Iron Nuggets \\
\hline Hismelt, Ausmelt & Fine Concentrate & Pulv. Coal & $\$ 200$ & $\$ 100$ & Liquid Iron \\
\hline ACCAR, Grate Car & Green Balls & Pulv. Coal, Gas & $\$ 250$ & $\$ 100$ & DRI \\
\hline $\begin{array}{l}\text { Microwave Assisted Hot } \\
\text { Metal Production }\end{array}$ & $\begin{array}{l}\text { Green Balls or Iron Ore } \\
\text { Concentrate }\end{array}$ & Pulv. Coal or Gas & $\$ 48$ & $\$ 68$ & Liquid Steel \\
\hline
\end{tabular}

\subsection{Economic Feasibility of a 500,000 T/y Plant}

This section uses standard discounted cash flow analysis to estimate the potential profitability of the microwave steel making process. The basic procedure is to estimate the various costs and revenues that would be expected if the process be constructed into a full scale operation. These estimates are used in a standard cash flow statement to calculate the net cash flow, which is then used to compute the net present value using an appropriate discount rate. Other measures of profitability such as rate of return can also be computed from the cash flows, but net present value method will be the measure of profitability to be used here. 
There are several possible applications for the microwave steel-making technology, and each of these will be analyzed as a different scenario.

Any measure of profitability of a project such as this relies entirely on estimates about costs, prices, and economic conditions in the future. It is of course impossible to know today exactly what these conditions will be, thus it is necessary to make forecasts and estimates. There are many different forecasting procedures, but almost all of them rely on using information from the past to choose values for the future. In other words, the past is usually used as a guide for the future. This may or may not lead to "good" forecasts of the future, but it is typically better than making completely uniformed guesses and there is usually no other procedure. The problem of estimating these future values is especially difficult when projecting out 15 or 20 years, as is necessary in this case because major capital plant investments must be for the long term.

\section{Basic Assumptions}

\section{Applications to be analyzed}

The two applications of microwave steel-making technology to be analyzed are:

A) Using the microwave technology to make hot metal for an existing mini-mill. This new technology would provide additional steel to the existing casting, rolling, and fabrication facilities. The purpose would be to provide another source of iron produced from iron ore to augment the existing electric arc furnace and its reliance on iron from scrap. In this scenario, the product from the microwave process would be hot metal, and the economic evaluation is for only the production of hot metal, without considering the fabrication facilities.

B) Constructing a stand-alone facility that would produce finished steel ingots that would compete against other steel in the semi-finished market. This scenario includes not only the microwave steel making furnace, but also the steel making and casting operations.

Figure 6.1 shows the basic inputs and materials flow for these two situations. Application A would only include the hot metal operations, whereas Application B would include hot metal, steel making and casting operations. In both cases, the analysis is based on producing $1 / 2$ million tones per year of hot metal from the microwave steel-making process. For the complete process of application B, the addition of other inputs such as some scrap and other steel-making ingredients results in the final production of 0.585 million tones per year of steel slabs. 


\section{Figure 6.1. Material Flows in Microwave Steel Making}

Hot Metal Operations:

\section{Iron Ore}

1.35

Coal

0.32

Electricity

667,233

\section{Steel Making: \\ Hot Metal}

0.83

Scrap

0.21
Tonnes concentrate per tonne metal

Tonnes coal per tonne metal

Kwhr per tonne metal
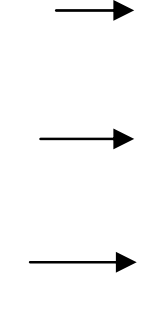

Million Tonnes

per year

Tonnes Iron Ore

0.160

333,617

$\sqrt{l}$

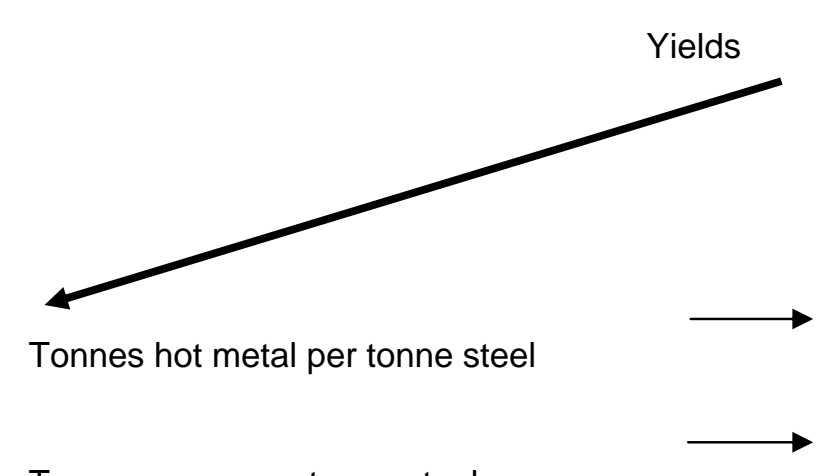

Tonnes scrap per tonne steel

$\sqrt{l}$

0.127

Tonnes Hot

Metal

Produced per year

Tonnes Hot

Metal

Tonnes Scrap

Metal

0.602

Tonnes Steel

Produced per

year

Tonnes Stee

Slabs

Produced per year 


\section{$\underline{\text { Technical parameters }}$}

The microwave steel making technology has been described elsewhere in this report, and Figure 6.1 shows the relevant technical parameters that are needed to estimate the costs and revenues from each scenario. Figure 6.1 shows the physical materials and energy flows for the process developed by this research. Table D.3A (in Appendix D) uses this information to develop the basic costs and revenues for the hot metal only operation of application A. Table D.3B (in Appendix D) uses the same information but extends the estimates to include the steel making and casting operations of application B.

All of this information applies to the first year of operation. The cash flow analysis will be extended out for an assumed plant life of 10 years. Many of these basic parameters will remain unchanged over the 10 years, such as the output of $1 / 2$ million tones per year of hot metal and the energy and other input requirements. However, many of the economic factors will change over time due to inflation or other anticipated economic fluctuations.

The data presented in Tables D.3A and D.3B represent the base case scenario since this is believed to be the most likely plant configuration and operating characteristics for the two applications. However, sensitivity analysis will be performed by varying some of the more important of these basic assumptions to determine how sensitive the overall profitability is to these assumptions.

\section{$\underline{\text { Economic and cost assumptions }}$}

This section discusses the key economic assumptions and describes the sources for the data.

\section{Economic Parameters for producing hot metal: Table D.3A}

Steel product selling price: This is the value per tone that is expected to be earned from producing hot metal. Since this replaces other sources of hot metal in the mini-mill, its value is the cost of obtaining hot metal via other means, such as melting of scrap. From source we estimate this value to be $\$ 300$ per tone.

Steel price escalation rate: This is the expected percentage change per year (the rate of change) in the product price. This is like an inflation rate except that it is specific to the price of the final product, and therefore is more properly referred to as the escalation in price. For much of the past, materials prices such as steel and metals have not increased at the same rate as the general rate of inflation, thus it is appropriate to use a different escalation rate for this than the general rate of inflation that is used for the other cost items.

Royalty rate: We are assuming that a royalty will be paid to the owners of the intellectual property rights of this process. We are assuming $1 \%$ of the gross revenue will be paid as royalties.

Cost inflation rate: This is the general rate of inflation that will occur over the life of the project, in percent per year. This rate of inflation applies to the costs of inputs, salaries and wages, and 
the various other expenses of the operation. We are using $2 \%$ per year, based on the inflation experienced in the US economy over the last few years.

Iron ore cost per tonne: This is the cost of purchasing iron ore concentrates as feedstock for the microwave furnace. This is obtained as an approximate average of recent iron ore prices as published in Skilling's Mining Review in the spring of 2003. These published prices need some translation, since they are published in terms of cents per natural iron unit and need to be converted into dollars per tone, based on the typical iron units per ton for the specific type of ore listed. These conversions were made for several major ore types which were then averaged together and rounded to $\$ 40.00$ per tonne.

Electricity cost per $\boldsymbol{k W h r}$ : This is the typical price per kilowatt-hour of electricity for a very large industrial customer using long term contracts. We found that some such customers were obtaining electricity at about 3.5 cents per kilowatt-hour in the spring of 2003.

Coal price per tonne: Coal prices were obtained from information published by Energy Publishing, Llc. In their "Coal \& Energy Price Report” from the spring of 2003. Since these prices vary somewhat depending upon the terms of delivery, quality and type of coal, and the market cycle, $\$ 20.00$ per tonne is used as a typical value.

Labor wage rate: The US Department of Labor publishes surveys of wages paid to workers in the iron and steel industries in the US and these show that the average wage for workers is about $\$ 25.00$ per hour.

Employee overhead rate: The same Department of Labor studies show that most industries of this type incur costs of about 55\% of salaries and wages for additional employee expenses such as unemployment insurance, heath insurance, retirement expenses, various kinds of employment taxes, and a variety of other costs associated with employing people.

\section{Base Case Analysis}

Table D.4 in Appendix D uses the base case assumptions for all the technical, cost, and economic parameters to compute the net cash flow for the two alternative situations. A project life of 10 years is assumed. Table D.4A shows the cash flow results and net present value results for producing only hot metal that would be used as feed material for an existing electric arc steel making operation. Table D.4B shows the corresponding analysis for a stand alone operation that would use the microwave process along with other facilities to produce cast steel slabs as the final product.

The results of each economic evaluation are summarized at the bottom of each table. The most important single number is the net present value: \$262 million in the case of producing only hot metal and \$316 million for producing steel slabs. Both of these are positive indicating that the projects are acceptable, given the assumptions used. More details about the specific cost items are shown below, and all of these estimates include the effects of the time value of money (cost of capital). 


\section{Sensitivity Analysis}

All of these results are of course critically dependent upon the assumptions used. In order to determine which of the specific assumptions are most critical towards affecting the profitability, sensitivity analysis was performed. Each critical assumption was varied by plus or minus $1 \%$ from the base case estimate, resulting in a new net present value. The percentage change in the net present value that resulted from this $1 \%$ change in the underlying parameter was then computed. This is the elasticity of net present value with respect to the given parameter. For example, if the cost of electricity were to increase by $1 \%$, this changes the net present value by $0.16 \%$, where a negative indicates a reduction in net present value.

Table 6.2 shows the elasticities for the key parameters and assumptions, where Table D.5A in Appendix D shows the results for producing only hot metal and Table D.5B shows the results for producing steel slabs. Other parameters and assumptions are even less important than these, thus they are not shown in this table. Clearly, the product selling price is by far the most critical factor for both alternative production strategies and the metal production rate is the second most important factor. For all of the other parameters and assumptions, a 1\% change results in less than a $1 \%$ change in final profitability.

\begin{tabular}{|r|c|c|}
\hline \multicolumn{3}{|c|}{ Table 6.2: Sensitivity analysis } \\
\hline & for producing only hot metal & for producing steel slabs \\
\hline Factor: & Elasticity: & Elasticity: \\
\hline Hot metal production rate & 1.07 & 1.12 \\
\hline Steel product selling price & 2.23 & 2.88 \\
\hline Iron ore cost per tonne & -0.42 & -0.35 \\
\hline Electricity cost per kwhr & -0.19 & -0.16 \\
\hline Coal cost per tonne & -0.06 & -0.05 \\
\hline Labor wage rate & -0.04 & -0.08 \\
\hline Scrap cost & & -0.19 \\
\hline Capital cost per tonne hot metal & -0.50 & -0.40 \\
\hline Capital cost per tonne steel & & -0.19 \\
\hline Capital cost per tonne slab & & -0.19 \\
\hline Cost of Capital: MARR & -0.69 & -0.82 \\
\hline
\end{tabular}




\section{Task 7. Evaluation of Policies, Regulations, and Affected Agencies}

The steel sector is a heavily-regulated industry that must abide by policies and rules of three federal agencies and their delegated state authorities: U.S. Environmental Protection Agency (EPA), Occupational Safety \& Health Administration (OSHA), and U.S. Department of Transportation (DOT). The major regulations of the EPA are the most comprehensive, covering air pollution, water pollution and waste disposal, and the Agency also spent much of the 1990s focusing on regulatory reform and reinvention activities. OSHA has a voluntary standard for occupational exposure to non-ionizing radiation, including microwaves. OSHA's primary regulations affecting this industry are its general safety and health regulations and its control of occupational exposure to cadmium. The DOT regulates the transportation of hazardous waste. While several other regulations apply to the steel industry, it should be noted that since microwave steel making technology has not yet been commercialized there are no specific, enforceable regulations for it. Consequently, we will discuss the primary regulations and policies affecting the electric arc furnace in the steel industry and speculate about the applicability of a few other regulations to microwaves. In most cases our proposed novel direct steel-making technology will be much more energy-efficient with substantially lower emissions and wastes than existing technology and have an easier time in meeting the applicable regulations.

\section{U.S. Environmental Protection Agency (EPA)}

The EPA's regulatory framework is generally organized around individual environmental media, i.e. air, water and land (for solid and hazardous wastes), and operates under more than a dozen statutes addressing these and other problems. Thus industrial sectors such as iron and steel must comply with a wide range of regulations as its operations affect multiple media. While coke ovens have traditionally been the largest environmental problem of this industry (the majority of environmental compliance costs are for air pollution control), environmental regulations affect it throughout all stages of manufacturing and product forming processes. Nonetheless, steel mills are primarily affected by four major environmental laws: the Clean Air Act (CAA), Clean Water Act (CWA), Resource Conservation and Recovery Act (RCRA), and the Emergency Planning and Community Right-to-Know Act (EPCRA). Other environmental statutes, such as the Comprehensive Environmental Response, Compensation, and Liability Act (CERCLA) and the Safe Drinking Water Act (SDWA) affect only a limited number of existing or former steel mills and will not be reviewed here (EPA, 1995). In addition, greenhouse gas emissions such as carbon dioxide $\left(\mathrm{CO}_{2}\right)$, while important, have yet to be regulated in the U.S. and thus steel mills do not face mandatory controls.

\section{$\underline{\text { Clean Air Act }}$}

The Clean Air Act of 1970, as amended in 1977 and 1990 (CAA), has required substantial capital investments to be made by the steel industry. While overall environmental control accounts for about $15 \%$ of total capital investment expenditures for the electric arc furnace industry, clean air requirements have accounted for more than three-fourths of the total in this sector (Wrona and Julien, 1997). Especially critical have been the state implementation plans (SIPs) to meet the National Ambient Air Quality Standards (NAAQS), particularly for nitrogen oxides $\left(\mathrm{NO}_{\mathrm{x}}\right)$, lead, and particulate matter. For steel mills with basic oxygen furnaces the largest 
challenge has been in keeping coke ovens in compliance with EPA standards, though dust control from electric arc furnaces is also critical.

The EPA promulgated two new NAAQS in 1997, for ozone $\left(\mathrm{O}_{3}\right)$ and particulate matter of 2.5 microns in diameter $\left(\mathrm{PM}_{2.5}\right)$ that are relevant to the steel industry. While controversial, the new standards were subjected to lawsuits but upheld by the U.S. Supreme Court on February 27, 2001. These NAAQS are expected to result in an increased number of areas in the country being designated with non-attainment (NA) status, once all the requisite rules are implemented and the monitoring equipment is in place and working properly (Brownell and Goldberg, 2001). The new standard will be an 8-hour average of $0.08 \mathrm{ppm}$ for $\mathrm{O}_{3}$, an annual $\mathrm{PM}_{2.5} \mathrm{NAAQS}$ of $15 \mathrm{ug} / \mathrm{m}^{3}$, and a daily $\mathrm{PM}_{2.5}$ NAAQS of $65 \mathrm{ug} / \mathrm{m}^{3}$. The preexisting $\mathrm{O}_{3}$ standard has been $0.12 \mathrm{ppm}$ averaged over 1 hour, while the $\mathrm{PM}_{2.5}$ standard is new (the preexisting coarse particulates standard, or $\mathrm{PM}_{10}$, of $50 \mathrm{ug} / \mathrm{m}^{3}$ and $150 \mathrm{ug} / \mathrm{m}^{3}$ for a year and daily, respectively, will continue to be in effect). While the NAAQS do not directly regulate stationary sources such as steel mills, if geographic areas are in NA status further restrictions on these sources may be required.

Title $\mathrm{V}$ of the CAA sets the permit requirements for major stationary sources of air pollution such as steel mills. A permit is required for facility construction, as well as its operation (for new, modified or existing sources). A Title $\mathrm{V}$ operating permit includes all CAA control requirements for a source in a single document and is legally enforceable by the EPA or a state air pollution control agency (40 CFR 70 and 71). These rules also establish a federal permitting program for use where state air agencies fail to establish or implement an adequate program, though most states are running their own air permitting program (since they meet the minimum EPA standards). Nevertheless, only about 6,000 operating permits have been issued thus far as compared to nearly 20,000 that are needed.

The control requirements for the criteria air pollutants regulated by the NAAQS for a major stationary source such as a steel mill vary depending on whether the facility is an existing, new, expanded or modified source, and whether it is located in a NA area or a geographic area in compliance. Existing facilities located in NA areas would have to install reasonably available control technology (RACT) as determined by the state authority, while no such requirements are imposed in the cleaner areas.

New Source Review (NSR) requirements under the CAA apply to major sources of the criteria air pollutants with new facilities, expansions or major process modifications (40 CFR 52.21(b)(1)(I)(a)-(b)), and is currently under review by the EPA. At the present time, a NSR for a facility in a NA area would by done by the state agency in most cases, and would require the facility to meet the lowest achievable emission rate (LAER) standard and acquire tradeable emission offsets of the same pollutant from other sources in the NA area in slightly greater amounts than the emission level of the new source (EPA, 1995).

A new steel mill to be located in an area meeting the NAAQS would be subject to a prevention of significant deterioration (PSD) review and would be required to install the best available control technology (BACT). BACT is the strictest possible technology standard required under the CAA, and is often equivalent to the LAER in practice. In addition, the owner or operator of the new source is required to conduct continuous on-site air quality monitoring in the area for a 
year prior to commissioning the new source to determine its effects on ambient air quality (EPA, 1995). To avoid this delay in startup in these clean areas, some mills have attempted to be reclassified as a "synthetic minor" source, which would require an even tighter restriction on total emissions allowed on the air permit, but thereby avoiding the lengthy and expensive PSD review.

The minimum standards for the LAER and BACT for iron and steel mills were set in 1975 by the EPA (40 CFR 60, Subpart AA), and last amended in 1999. The New Source Performance Standards (NSPS) for electric arc furnaces are described in 40 CFR 60.270, which regulates the particulate matter and opacity in any gases discharged from dust-handling equipment. The standard for particulate matter is $12 \mathrm{mg} / \mathrm{dscm}$, which is usually controlled with a baghouse. The shop opacity (the arithmetic average of 24 or more opacity observations of emissions from the shop taken in accordance with EPA Reference Method 9) limit is under 6\% during melting and refining, 20\% during charging, and 40\% during tapping. A continuous emission monitoring system is required for the measurement of opacity.

The EPA has issued several Maximum Achievable Control Technology Standard (MACT) standards for emissions of hazardous air pollutants (HAPs) that affect the steel industry, which sometimes is also called National Emission Standards for Hazardous Air Pollutants (NESHAPs) under Title III of the CAA. The MACT treatment standard is defined as a technology that will result in the maximum degree of reductions achievable subject to economic, energy and environmental considerations. Such standards have been set for coke oven batteries; benzene emissions from coke by-product recovery plants; halogenated solvent cleaning; chromiumindustrial process cooling towers; steel pickling facilities that use hydrochloric acid; and have been proposed for integrated iron and steel manufacturing facilities with blast furnaces and basic oxygen process furnaces. While the EPA had once also considered establishing a MACT standard for electric arc furnaces (EAF), it decided to de-list this category on June 4, 1996 (61 FR 28197). This was done because the EPA believes that there are no major EAF sources that emit more than 10 tons per year of a single HAP, or 25 tons per year of the sum of all HAPs emitted per year. In addition, the EPA does not believe that the EAF technology poses a significant risk warranting a standard that would affect area sources.

The CAA required the EPA to promulgate regulations to prevent accidental releases of regulated substances and to reduce the severity of those releases that do occur. Pursuant to this requirement, under section 112(r)(7) of the CAA (“Accident Prevention”) stationary sources with processes that contain more than a threshold quantity of a regulated substance have been required to prepare and implement a Risk Management Plan (RMP). The EPA issued its list of 77 regulated toxic substances and 63 flammable substances and threshold quantities on January 31, 1994, which affects many steel mills including mini-mills such as Nucor. The regulation for risk management programs and the RMP was issued on June 20, 1996, and facility RMPs were required to be filed and registered with the EPA by June 21, 1999. About 15,000 RMPs were received by the EPA across all sectors. The RMPs also were required to be submitted to the five member Chemical Safety and Hazard Investigation Board, and be available to state and local authorities, and the general public (40 CFR 68). 
Processes are divided into three program categories, based on the potential for offsite consequences associated with a worst-case accident release; no offsite accident history; or compliance with the prevention requirements under OSHA's Process Safety Management (PSM) Standard (29 CFR 1926). Processes that have no potential impact on the public in the case of an accident release have minimal requirements. For other processes, sources must implement a risk management program that includes more detailed requirements for hazard assessment, prevention, and emergency response. Processes in industry categories with a history of accidental releases and processes already complying with OSHA's PSM Standard are subject to a prevention program identical to parallel elements of the OSHA Standard. All other processes are subject to a streamlined prevention requirement. All sources, however, must prepare offsite consequence analyses for a worst-case release scenario.

An RMP must include the following information:

- a 5-year history of accidental releases of substances subject to the RMP regulation that have resulted in injury or death to humans, off-site evacuations, or property or environmental damage;

- a hazard assessment of worst-case releases of toxic or flammable substances and, for some sources, alternative release scenarios;

- $\quad$ a summary of the facility's emergency response program, including procedures for notifying the public of a release, and schedules for drills completed and planned;

- $\quad$ the contact person for emergencies;

- $\quad$ the date of the last safety inspection and the agency that performed it; and

- $\quad$ a general description of the company's accident prevention plan.

Some sources also need to include pertinent details of any incident investigation, management of change procedures, operating procedures, pre-startup reviews, maintenance activities, compliance audits, process safety training information, and process hazard analyses.

\section{$\underline{\text { Clean Water Act }}$}

The Clean Water Act of 1972, as amended in 1977 and 1987 (CWA), set up the National Pollution Discharge Elimination System (NPDES) for permitting and controlling effluents from point sources of water pollution. Most NPDES permitting programs are implemented by state environmental agencies. Since the iron and steel manufacturing industry is a major water user, the EPA established effluent limitations guidelines and standards for it in a 1974 regulation, revised in 1982 and amended in 1984, for both new and existing facilities (40 CFR 420). These guidelines and standards are implemented through the NPDES permit program and through state and local pretreatment programs. Whereas part 420 limits in the EPA regulations are productionbased, steel mills with higher levels of output receive higher effluent discharge allowances in their permits. Subpart D, in particular, covers steelmaking including electric arc furnaces (EPA, 1995). These point sources of water pollution are required to install best practicable technology currently available (BPT) or best available technology economically achievable (BAT).

Revisions to the effluent limitations guidelines and pretreatment standards for wastewater discharges were proposed by the EPA in August, 2002 (EPA, 2000). Among the proposed 
changes would be a zero discharge standard as the NSPS at non-integrated steel mills, and elimination of effluent trading for oil \& grease (see the discussion on effluent trading below).

The water pollutants covered by these regulations fall into three categories:

1. Conventional pollutants: total suspended solids, oil \& grease, $\mathrm{pH}$;

2. Nonconventional pollutants: ammonia, phenols; and

3. Priority (toxic) pollutants: cyanide, chromium (total and hexavalent), lead, nickel, zinc, benzene, benzo(a)pyrene, napthalene, tretachlorethylene.

The EPA has worked with steel companies to try and reduce the volume and toxicity of wastewater effluents discharged from their mills. Water pollution from the steel industry has also been the subject of several court challenges by environmental groups. Wastewater effluent is often recycled at steel mills to reduce the volume of such discharges. In addition, the process wastewater is typically filtered or clarified on-site before it is discharged. The oil and greases are removed from the process wastewater by either skimming, filtration or air flotation, and are often used as lubricants or for preservation coatings. Some waste iron and organic chemicals will remain in the sludge, which can be recovered and reclaimed through sintering and pelletizing operations (EPA, 1995).

To increase the flexibility and cost-effectiveness of effluent control at steel mills, the EPA has allowed intra-plant effluent trading at Sparrows Point, Maryland and at about 10 other iron and steel mills in Michigan and Indiana since 1983, although most of the trades are no longer active (Podar and Kashmanian, 1998). This permitting option resulted from a negotiated settlement between the Natural Resources Defense Council and the iron and steel industry. Trading has been permitted for conventional pollutants (total suspended solids and oil \& grease) as well as toxic pollutants (zinc and lead). To have intra-plant trading approved, the steel mills have had to reduce their conventional pollutants by at least $15 \%$ and the toxic pollutants by at least $10 \%$ below the BPT or BAT limits, although the net water quality effects of such trading have not been clear (Kashmanian et al., 1995).

The storm water rule under the CWA (40 CFR 122.6(b)(14), subparts (i, ii)) requires the capture and treatment of storm water at primary metals manufacturing facilities, including iron and steel plants. The management of storm water reduces the discharge of total suspended solids, biological oxygen demand (BOD), oil \& grease, and some metals (EPA, 1995).

\section{$\underline{\text { Resource Conservation and Recovery Act }}$}

Under Subtitle C of the Resource Conservation and Recovery Act of 1976 (RCRA), as amended in 1984 (40 CFR 261), generators of hazardous wastes are responsible for keeping track of the wastes they generate and where they go for treatment, storage or disposal. Electric arc furnace emission control dust and sludge are identified by the RCRA as process K061, although $90 \%$ of these wastes are managed for metal recycling or recovery (EPA, 1995). The metals targeted for recovery are iron, zinc, nickel alloys, lead, and cadmium. This statute requires "cradle to grave" waste management and restricts land disposal at landfills and includes waste accumulation, manifesting, and record keeping standards. Consequently any hazardous wastes shipped off-site 
must be accompanied by a hazardous waste form or manifest at all times, to ensure that the transportation and disposal of the wastes is done in an environmentally sound manner. Forty-six of the 50 state environmental agencies implement the RCRA provisions.

Several technical options exist for the disposition of the metals in the dust and sludge that are not recycled. These include stabilization, use as an ingredient in fertilizer, cement production, or in glass grit for abrasive blast, glass ceramic or ceramic glaze, roofing shingles, and use as an ingredient in the production of special aggregates.

The EPA proposed a conditional exclusion from the definition of solid waste for hazardous secondary materials that are recycled to make zinc fertilizers or their ingredients (65 Federal Register 70954, November 28, 2000). A generator of zinc waste would no longer be subject to hazardous waste management regulations as long as it meets the specified conditions relating to accumulation, storage, transportation, reporting and record-keeping requirements of excluded materials. This proposal included a second conditional exclusion for zinc fertilizers made from hazardous wastes or excluded hazardous secondary materials (p. 70967). Manufacturers would be required to meet the proposed technology-based contaminant limits, and maintain analytical data and analyses demonstrating compliance.

The EPA also is pursuing a Hazardous Waste Identification Rule exemption, which would exempt listed hazardous wastes that meet chemical-specific exemption levels from the definition of hazardous wastes. While this rule was originally planned to be issued in April 2001, its release has been delayed because of complexities involved in the risk assessment using the Multi-media, Multi-pathway and Multi-receptor risk assessment (3MRA) Model. This rule would be intended to better align the costs of RCRA regulation with the risks being controlled.

\section{Emergency Planning and Community Right-to-Know Act}

Under Section 313 of the Emergency Planning and Community Right-to-Know Act (EPCRA), a steel mill is subject to the annual reporting requirements if it has 10 or more full-time employees and it manufactures, imports, processes or otherwise uses any of the EPCRA section 313 chemicals an amounts greater than threshold quantities. There are currently more than 650 chemicals and chemical categories on this list. Examples of target chemicals at steel mills with electric arc furnaces are cadmium, lead, zinc, and hexavalent chromium. The threshold reporting quantities are usually 25,000 pounds per chemical per year for target chemicals that are manufactured or processed at the facility, and 10,000 pounds per chemical per year for target chemicals that are otherwise used at the facility. De minimus reporting exemptions are allowed when these chemicals are less than 0.1 percent or 1 percent (depending on the chemical in question) in mixtures.

While most (if not all) steel mills with electric arc furnaces operate below the reporting thresholds of the EPCRA these facilities should still be familiar with the reporting requirements. The information requested under section 313 of the EPCRA must be filed on Form R, available from the EPA, for each of the Toxic Release Inventory (TRI) chemicals above the threshold and de minimus quantities listed by the EPA. The EPA consolidates these reports on its annual TRI report. Each company must report the following: 
- $\quad$ the name and location of the facility;

- $\quad$ the identity of the EPCRA section 313 chemical;

- $\quad$ whether it manufactures, imports, processes, or otherwise uses the chemical;

- $\quad$ the maximum quantity of the chemical on-site at any time during the year;

- the total quantity of the chemical released during the year (separately for on-site releases to air, water, land and injected underground; and transfers for off-site disposal;

- the total quantity of the EPCRA section 313 chemical otherwise managed as waste during the year (separately for on-site treatment, on-site combustion for energy recovery, on-site recycling, transfers for off-site treatment, transfers for off-site energy recovery, and transfers for off-site recycling):

- $\quad$ off-site locations that were shipped wastes containing the EPCRA section 313 chemical and the quantity of that chemical sent to those locations for recycling, energy recovery, treatment, or disposal;

- on-site recycling, energy recovery, or treatment methods used for wastes containing the EPCRA section 313 chemical, and estimates of the treatment efficiency for each chemical;

- $\quad$ source reduction activities involving the EPCRA section 313 chemical.

\section{Common Sense Initiative}

The Common Sense Initiative (CSI) was an innovative experiment conducted from 1994-98 to encourage "cleaner, cheaper, and smarter" sector-based approaches to protecting the environment and human health. It was a primary component of the EPA's regulatory reinvention efforts in the 1990s. The EPA addressed six sectors in this initiative; iron and steel was one of them. The Iron and Steel Subcommittee included more than 20 representatives from industry, environmental justice organizations, labor and environmental groups, and federal, state and local governments. This Subcommittee met several times between January 1995 and May 1998. Using a consensus approach to decision making intended to avoid costly delays and litigation, the subcommittee considered a variety of topics including regulation, permits and public participation, compliance, reporting, Brownfields, pollution prevention and environmental technology. The CSI Council presented 28 formal recommendations from all subcommittees to the EPA at the end of 1998, few of which have been implemented thus far (GAO, 1997).

Several projects were conducted by the CSI Iron and Steel Subcommittee, with mixed results. For example, state/EPA multi-media (air, water, wastes) permit information was identified that can be consolidated into electronically submitted reporting for a mini-mill. The Subcommittee also developed a pseudo, non-enforceable multi-media permit. The consolidated report can potentially reduce duplication, minimize errors, yield significant cost savings and provide affected communities with usable environmental information. This project has already been integrated into Utah's "One Stop” reporting initiative. General permitting issues were also considered. Two recommendations resulted in an improved NSPS regulation for electric arc furnace monitoring (issued on March 2, 1999), and consolidated guidance on witnessing certain air testing, respectively. Another important project resulted in the establishment of an iron and steel (among other sectors) liaison at the EPA. This liaison is currently Robert S. Benson, director of the Sector Strategies Division in the Office of Policy, Economics and Innovation. 
While the CSI formally ended in 1998, important follow-up work is being done as part of the EPA’s sector programs.

\section{Occupational Safety \& Health Administration (OSHA)}

The safety and occupational health of workers in steel mill is regulated under the Occupational Safety and Health Act of 1970. This Act encourages safe and healthful working conditions, and authorizes the Secretary of Labor to set mandatory occupational safety and health standards and enforce them through OSHA. OSHA has established permissible exposure limits (PELs) for about 430 chemical substances that are considered occupational air contaminants, which are listed in 29 CFR 1910.1000. The PELs include time-weighted average (TWA) limits, short term exposure limits, ceiling limits and, in some cases, skin designations.

OSHA promulgated a standard for occupational exposure to cadmium, which took effect in December 1992 (29 CFR 1910.1027). Cadmium is part of the hazardous waste stream contained in the dust produced by the operation of an electric arc furnace in a steel mill. Employees exposed to excess amounts of cadmium face a significant health risk in the form of lung and prostate cancers, and serious kidney dysfunction. OSHA's standard is an 8-hour time TWA PEL of 5 micrograms of cadmium per cubic meter $\left(\mathrm{ug} / \mathrm{m}^{3}\right)$ of air, which includes exposure through fume and dust. Employers are required to comply with this PEL primarily by means of engineering and work practice controls.

For microwave operations, the primary health effects of concern from electromagnetic radiation are thermal exposure or burns and contact shocks, although research is continuing to investigate non-thermal effects. OSHA has voluntary standards for workplace exposure to non-ionizing radiation (including microwaves) that can be found in 29 CFR Section 1910.97, which were approved in 1996. The recommended power density exposure limit is $10 \mathrm{milliwatt} / \mathrm{cm}^{2}$ for periods of 0.1 -hour or more. An energy density limit of 1 milliwatt-hour $/ \mathrm{cm}^{2}$ during any 0.1 hour period is also recommended. These standards have been ruled unenforceable. Some U.S. states with their own OSHA-type programs, however, are enforcing this or other exposure limits. For instance, while Michigan follows the OSHA guidelines the New Jersey Department of Environmental Protection has an exposure limit of 5 milliwatt $/ \mathrm{cm}^{2}$. The guideline is also half the OSHA level in Ontario, Canada (Ontario Ministry of Labour, 2002). The OSHA standard also specifies the design of a warning sign, although the inclusion and choice of warning information or precautionary instructions is discretionary.

\section{U.S. Department of Transportation (DOT)}

Electric arc furnace dust typically contains $1-2 \%$ of the charge in the form of zinc, lead, cadmium, chromium and smaller amounts of other hazardous materials. While there is a modest but growing market for recovery and use of these hazardous wastes by-products, especially zinc, their shipment is subject to the regulations under the Federal Hazardous Materials Transportation Law (49 CFR 171-180). These regulations are issued by the Research and Special Programs Administration of the U.S. Department of Transportation (DOT). The rules and regulations govern the safe transportation of hazardous materials from all sources; authorize approvals and exemptions for specific activities; require the receipt and maintenance of records of cylinder test 
reports, manufacturer certifications, and incident reports; normally preempt state, local and tribal hazardous materials transportation requirements; and provide for civil and criminal penalties for violations. 


\section{ACCOMPLISHMENTS}

After three years of effort, the bench scale study has been successfully completed and the technical feasibility of microwave steelmaking has been verified. Energy efficiency, cost savings, and environmental benefits of the revolutionary steelmaking technology has indicated a potential savings of $45 \%$ of the energy and a $40 \%$ reduction in steel production costs. Environmental benefits are significant, including the reduction of particulates, $\mathrm{CO}_{2}, \mathrm{SO}_{2}, \mathrm{NOx}$, VOCs, CO emissions, and slag generation.

U.P. Steel has licensed this technology for commercialization. This is a private company created in 2001. The founders of the company have extensive experience in mining, mineral processing and steelmaking, and identified microwave steelmaking as the most important and promising technology for the future of the industry. U.P Steel has filed world wide patent applications and extensively marketed the process with $\$ 100,000$ of current investment.

Cleveland Cliffs Iron Company is the largest domestic iron ore company and operates iron ore mines in Michigan. CCI participated in this microwave steelmaking project by providing iron ore for steelmaking tests and assistance in evaluating test results and economics. CCI has closely watched the progress of the microwave steelmaking technology since 2001 and is actively involved in the proposed pilot plant planning and cost sharing.

Several presentations were made during the project:

Potential Application of Microwaves in Steelmaking

Third World Congress on Microwave and RF Applications

Microwave Working Group, September 22 - 26, 2002, Sydney, Australia

Authors: S. X. Huang, J. Y. Hwang, S. Shi, R. C. Greenlund, T. Xu, and A. M. Hein

Microwave Assisted Direct Steelmaking

Upper Peninsula Section of SME, Annual Technical and Business Meeting

Michigan Technological University, Houghton, MI

April 16, 2003

$X$. Huang

Microwave Production of Steels

Structural Engineers Association of Texas, 2004 State Conference

Lakeway, TX,

October 21-23, 2004

J.Y. Hwang, X. Huang

Contributing investigator, Michigan Tech Associate Professor Barry Solomon (Social Sciences) presented a paper, "A Market Transformation Strategy for Highly Efficient Steel Meeting," at the 2003 Summer Study on Energy Efficiency in Industry of the American Council for an Energy-Efficient Economy, which was held from July 29-Aug. 1, 2003 in Rye Brook, New York. 
One PhD dissertation was completed during the project period: "Microwave Application and Modelling in Direct Reduction of Iron Oxide” by Shaolong Qu, in partial fulfillment of the requirements for a degree of Doctor of Philosophy in the field of Chemical Engineering, Michigan Technological University, 2004.

Demonstrations to at least ten sets of visitors interested in the microwave steelmaking technology were given over the course of the project, including Noramco, US Steel, Steel Dynamics, Cober Electronics, CCI, Michigan State government Representatives, automobile foundries, and foreign industries. 


\section{PROJECT GENERAL CONCLUSIONS}

From the conclusions made in each task, we have the following general conclusions for this Phase I project.

- MW/EAF steelmaking is a validated technology. Its technical feasibility was proven by a previous concept study and has been proven again by the newly developed bench scale equipment and the bench scale study. Evaluation of the produced steels showed that steel containing $0.2 \%$ carbon and less than $0.01 \%$ sulfur can be produced. Steel yield above $95 \%$ can be easily obtained.

- MW/EAF steelmaking is a much more environmental friendly process than current technologies. Environmental benefits are significant, including the reduction of particulates, $\mathrm{CO}_{2}, \mathrm{SO}_{2}, \mathrm{NOx}, \mathrm{VOCs}, \mathrm{CO}$ emissions, and slag generation. The process reduces the environmental impact of steelmaking by preventing pollutant generation rather than relying on end-of-the-pipe pollution treatment.

- MW/EAF steelmaking is a more energy efficient process than current steelmaking processes using iron ore as the feed material. Results indicate a potential savings of $45 \%$ of the energy and a $40 \%$ reduction in steel production costs. In addition, iron ore reduction time is dramatically reduced.

- The inherent flexibility of the technology allows it to be used with various feedstocks and process sizes. This facilitates the integration of MW/EAF steelmaking with the current steelmaking plants and creates less disorder in the industry.

- MW/EAF steelmaking requires much lower capital cost and operation cost in comparison with current steelmaking processes. It is economically feasible and will provide more profit to the steel industry if it is implemented.

- MW/EAF steelmaking can be scaled up to the current high volume industry production using currently available manufacturing techniques to build the required new steelmaking equipment. 


\section{RECOMMENDATIONS}

While many of the scale-up and integration questions have been answered, there is still work to do to bring microwave steelmaking technology to commercial readiness. Larger scale testing and integration studies should continue. We propose to design, build and test a $500 \mathrm{~kg} / \mathrm{h} \mathrm{MW} \mathrm{DRI}$ column with the feature of exporting gaseous fuel and associated material charge and discharge mechanisms. We also propose to study the integration of the MW DRI columns with an EAF through a conveyer system and define the plant layout.

The goal of the proposed Phase II study is to develop the key equipment for the MW/EAF steelmaking technology and make it ready for commercialization activities.

The objectives associated with the goal are to a) design and fabricate a $500 \mathrm{~kg} / \mathrm{h}$ MW DRI furnace with the feature of exporting gaseous fuel; b) test and evaluate its performance; c) generate a solid base of technical and economic data for the next phase of development; e) assess energy efficiency and environmental impact of the $500 \mathrm{~kg} / \mathrm{h}$ MW DRI furnace and overall MW/EAF steelmaking based on the test data; f) define industrial implementation of MW/EAF steelmaking process; and g) demonstrate the pilot equipment performance to potential investors to promote an early industrial implementation. 


\section{REFERENCES}

- K. E. Haque, "Microwave Energy for Mineral Treatment Processes: a Brief Review”, Int. J. Miner. Process., volume 57, 1999

- J. E. Gerling, "Microwave Oven Power: A Technical Review”, J. Microwave Power and Electromagnetic Energy, 28, 1987

- D. E. Clark, W. H. Sutton, "Microwave Processing of Materials", Annu. Rev. Mater. Sci., volume 26, 1996

- L. Chunpeng, X. Yousheng, H. Yixin, “Application of Microwave Radiation to Extractive Metallurgy”, Chin. J. Met. Sci. Technol., volume 6, 1990

- J. Jacob, L. Chia, F. Boey, "Thermal and Non-thermal Interaction of Microwave Radiation with Materials”, J. Mater. Sci., volume 30, n 21, 1995

- D. K. Xia, C. A. Pickles, "Applications of Microwave Energy in Extractive Metallurgy”, CIM Bulletin, volume 90, 1997

- Y. V. Bykov, K. I. Rybakov and V. E. Semenov, "High-Temperature Microwave Processing of Materials”, J. Phys. D: Appl. Phys., volume 34, n 13, 2001

- H. S. Shulman, "Microwaves in High-Temperature Processes”, Industrial Heating, volume 70, n 3, 2003

- M. A. Janney, "Enhanced Diffusion in Sapphire during Microwave Heating“, J. Mater. Sci., volume 32, n 5, 1997

- R. N. Gedye, J. B. Wei, "Rate Enhancement of Organic Reactions by Microwaves at Atmosphere Pressure“, Can. J. Chem., volume 66, 1988

- R. N. Gedye, F. E. Smith and K. C. Westaway, "Microwaves in organic and organometallic synthesis”, J. Microwave Power and Electromagnetic Energy, volume 26, n 1, 1991

- Y. V. Bykov, "Evidence for Microwave enhanced Mass Transport in the Annealing of Nanoporous Alumina Membranes”, J. Mater. Sci., volume 36, issue 1, 2001

- J. H. Booske, "Non-equilibrium Excitation of Mass Transport under Microwave Irritation”, J. Mater. Res., volume 7, 1992

- Ahmad, "Effect of Microwave Heating on the Solid State Reactions and Mass Transport in Ceramics", Dissertation Abstracts International, volume 52, n 10, 1992

- W. H. Sutton, "Microwave Processing of Ceramic Materials", Ceramic Bulletin, volume 68, n 2, 1989

- N. Standish and Pramusanto, “Reduction of Microwave Irradiated Iron Particles In CO”, ISIJ International, volume 31, n 1, 1991

- N. Standish and W. Huang, "Microwave Application in Carbothermic Reduction of Iron Ores”, ISIJ International, volume 31, n 3, 1991

- W. Wu and W. Huang, "Microwave Drying and Roasting of Pellets”, In “ Metallurgical Processes for Early Twenty-First Century", edited by H. Y. Sohn, TMS, 1994

- Gomez, J. A. Aguilar, "Microwaves for Reduction of Iron Ore Pellets by Carbon”, Materials Research Society Symposium Proceedings, Materials Research Society, volume 366, 1995

- S. Zhong, H. E. Geotzman and R. L. Bleifuss, "Reduction of Iron Ore with Coal by Microwave Heating”, Minerals \& Metallurgical Processing, volume 13, n 4, 1996

- C. Moore, R. I. Marshall, "Steelmaking”, The Institute of Metals, 1991

- O. Kubaschewski and E. LL. Evans, "Metallurgical Thermochemistry", 2nd edition, John Wiley \& Sons, INC - Pergamon Press LTD, 1956 
- N. Standish, H. Worner and G. Gupta, “Temperature Distribution in Microwave-Heated Iron Ore-Carbon Compositions”, J. Microwave \& Electromagnetic Energy, volume 25, n 2, 1990

- ASTM, "Standard Test Methods for Chemical Analysis of Soda-Lime and borosilicate Glass”, ASTM C 169-92, Annual Book of ASTM Standards, volume 15.02, 1992

- W. G. Wilson and A. McLean, "Desulfurization of Iron \& Steel and Sulfide Shape Control”, The Iron \& Steel Society of AIME. 1980

- H. Ushiyama, “Ladle Furnace Process in Japan”, Ironmaking and Steelmaking, volume 3, 1978.

- R. J. Fruehan, “The Making, Shaping and Treating of Steel”, 11th edition Steelmaking and Refining Volume, The AISE Steel Foundation, 1998

- R. J. Fruehan, et al. “Theoretical Minimum Energies to Produce Steel for Selected Conditions”, Report for the U.S. Department of Energy, Office of Industrial Technologies. 2000

- J. Stubbles, "Energy Use in The U.S. Steel Industry: A Historical Perspective and Future Opportunities”, Report for the U.S. Department of Energy, Office of Industrial Technologies. 2000

- $\quad$ N. Margolis, R. Brindle, "Energy and Environmental Profile of the U.S. Iron and Steel Industry”, Report for the U.S. Department of Energy, Office of Industrial Technologies. 2000

- “Annual Statistical Report 1998”, American Iron and Steel Institute (AISI), 1999

- H. E. McGannon, “The Making, Shaping and Treating of Steel”, 9th edition, United State Steel Corporation, Herbick \& Held, 1970

- J. Aguilar et al, "Simulation of Iron Ore reduction in a fixed bed”, Modeling Simul. Mater. Sci. Eng., volume 3, 1995

- W. M. McKewan, “Kinetics of Iron Oxide reduction”, Trans. TMS-AIME, volume 218, n 1, 1960

- J. M. Quets, M. E. Wadsworth and J. R. Lewis, "Kinetics of Hydrogen Reduction of Magnetite”, Trans. TMS-AIME, volume 218, n 3, 1960

- E. T. Turkdogan and J. V. Vinters, “Gaseous Reduction of Iron Oxides EM Dash 3”, Metallurgical Transactions, volume 3, n 6, 1972

- E. T. Turkdogan and J. V. Vinters, “Gaseous Reduction of Iron Oxides EM Dash 4”, Metallurgical Transaction, volume 3, n8, 1972

- Kobayashi, Y. Tanigaki, A. Uragami, “A New Process to Produce Iron Directly from Fine Ore and Coal”, Iron and Steelmaker, volume 28, n 9, 2001

- "What you should know about Industrial Microwave Processing,” Brochure of Microdry Inc., 7450 Hwy. 329, Crestwood, KY 40014. 11

- “Steel Technology Roadmap,” American Iron and Steel Institute, 1998. http://www.steel.org/mt/roadmap/roadmap.htm, 1999. 1

- Sven Eketorp, "Decisive Factors for the Planning of Future Steel Plants," Proceeding of the Third International Iron and Steel Congress, 16-20 April, 1978, Chicago, IL, pp. 181-185. 2

- Peter Kakela, "Pelletized vs. Natural Iron Ore Technology: Energy, Labor, and Capital Changes,” Energy Conservation, Oct., 1979, pp. 18-19. 13

- Maurice Y. Meunier and Oscar de Bruyn Kops, "Energy Efficiency in the Steel Industry with Emphasis on Developing Countries,” World Bank Technical paper, ISSN 0253-7494, No. 22, Industry and Finance Series, Vol. 4. 14 
- Michael R. Sanio and Philip S. Schmidt, "Economic Assessment of Microwave and RadioFrequency Materials Processing," Mat. Res. Soc. Symp. Proc., Vol. 124, 1988, p337. 15

- Irving J. Chabinsky, "Applications of Microwave Energy Past, Present and Future," Mat. Res. Soc. Symp. Proc., Vol. 124, 1988, p17. 10

- N. Standish and H. Worner, "Microwave Application in the Reduction of Metal Oxides with Carbon," Journal of Microwave Power and Electromagnetic Energy, Vol.25 No.3, 1990, p177. 6

- G. Bisio, "Energy Method for Efficient Energy Resource Use in the Steel Industry," Energy, Vol. 18, No. 9, 1993, pp. 971-985. 17

- R.W. Bouman, “Energy Requirements for Ironmaking and Steelmaking,” I\&SM, Jan., 1983, pp. 23-25. 18

- R. J. Fruedhan, “Evaluation of Steelmaking Processes,” DOE/ID12847-5 (DE94005368), Jan., 199. 19

- Dwight C. Brown, “Direct Reduction of Iron Ores - A 1961 Review,” Recent advances in ferrous metallurgy, Symposium Series 43, American Institute of Chemical Engineers, Vol. 59, 1963, pp. 53-83.

- Robert A. Lubker and Kenneth W. Bruland, "Fluidized Bed Processes for the Direct Reduction of Iron Ores," Recent advances in ferrous metallurgy, Symposium Series 43, American Institute of Chemical Engineers, Vol. 59, 1963, pp. 84-92.

- Mateo Quiroga and Hector Sanchez, "HYLSA 2P Plant,” Ironmakign Proceedings, Vol. 38, Detroit 1979, pp. 253-258.

- Shaolin Zhong and Harold E. Goetzman, "Reduction of Iron Ore with Coal by Microwave Heating,” Coleraine Mineral Research Laboratory Report, April 1996.

- J. M. Tranquilla and M. Gordon, “Microwave Treatment - Economic Sense,” ERM Microwave Technology Corporation, 64 Alison Blvd., Fredericton, N. B. Canada, reprint presented at Randol Gold \& Silver Forum 1998.

- J. M. Tranquilla, W. LaRochelle, and P. Brooks, "Design and Operation of a Microwave Pilot Plant for Calcining Refractory Gold Ores and Concentrates,” ERM Microwave Technology Corporation, 64 Alison Blvd., Fredericton, N. B. Canada, reprint, presented at Randol Gold \& Silver Forum 1998.

- "Annual Statistical Report", American Iron and Steel Institute, 1995.

- CRC Handbook of Chemistry and Physics, 71st edition, 1990-1991, edited by David R. Lide, p5-36.

- [AISI] American Iron and Steel Institute. 2001. Steel Industry Technology Roadmap. Washington, D.C.: AISI. . 2002. Annual Statistical Report: 2001. Washington, D.C.: AISI.

- Brown, M.A. 2001. "Market Failure and Barriers as a Basis for Clean Energy Policies." Energy Policy 29 (14): 1197-1207.

- Brown R., C. Webber, and J. Koomey. 2000. "Status and Future Directions of the Energy Star Program.” In Proceedings of the ACEEE 2000 Summer Study on Energy Efficiency in Buildings, 6.33-43. Washington: American Council for an Energy-Efficient Economy.

- Crompton, P. 2001. “The Diffusion of New Steelmaking Technology.” Resources Policy 27 (1): 87-95.

- de Beer, J., E. Worrell, and K. Blok. 1998. "Future Technologies for Energy-Efficient Iron and Steel Making.” Annual Review of Energy and the Environment 23: 123-205. 
- DeCanio, S.J. 1998. “The Efficiency Paradox: Bureaucratic and Organizational Barriers to Profitable Energy-Saving Investments.” Energy Policy 26 (5): 441-54.

- DeCanio, S.J., C. Dibble, and K. Amir-Atefi. 2000. “The Importance of Organizational Structure for the Adoption of Innovations.” Management Science 46 (10): 1285-99.

- Duke, R., and D.M. Kammen. 1999. “The Economics of Energy Market Transformation Programs.” The Energy Journal 20 (4): 15-64.

- [EIA] Energy Information Administration. 2001. Manufacturing Consumption of Energy, 1998. Washington, D.C.: U.S. Department of Energy.

- $\quad$ Friedman, N.R., R.N. Elliott, B. Meberg, J. Dowd, C.A. Burrell, and J.F. DeKorte. 1996. Electric Motor System Market Transformation. Washington, D.C.: American Council for an Energy-Efficient Economy.

- Fruehan, R.J. 1997. "Future Steelmaking Technologies and the Role of Basic Research." Metallurgical and Materials Transactions, Vol. 28B (5), 743-53.

- Geller, H. 2003. Energy Revolution. Washington, D.C.: Island Press.

- Kwasnicki, W., and H. Kwasnicka. 1996. "Long-Term Diffusion Factors of Technological Development.” Technological Forecasting and Social Change 52 (1): 31-57.

- Margolis, N., and L. Sousa. 1997. "Energy and Environmental Profile of the US Iron and Steel Industry.” In Proceedings of the ACEEE 1997 Summer Study on Energy Efficiency in Industry. Washington, D.C.: American Council for an Energy-Efficient Economy.

- McCane, A.T., V. Tutterow, and C. Cockrill. 2001. "Making Industrial Energy Efficiency Mainstream and Profitable: Where Public Benefit and Private Interests Intersect.” In Proceedings of the ACEEE 2001 Summer Study on Energy Efficiency in Industry. Washington, D.C.: American Council for an Energy-Efficient Economy.

- Oster, S. 1982. "The Diffusion of Innovation Among Steel Firms: The Basic Oxygen Furnace.” Bell Journal of Economics 13 (1): 45-56.

- $\quad$ Ruth, M., and A. Amato. 2002. "Vintage Structure Dynamics and Climate Change Policies: The Case of US Iron and Steel.” Energy Policy 30 (7): 541-52.

- Stubbles, J. 2001. “The U.S. Steel Industry: Energy Consumption and CO2 Emissions.” In Proceedings of the ACEEE 2001 Summer Study on Energy Efficiency in Industry. Washington, D.C.: American Council for an Energy-Efficient Economy.

- $\quad$ [DOE] U.S. Department of Energy. 2000. Clean Energy Partnerships: A Decade of Success. Washington, D.C.: U.S. Department of Energy, Office of Energy Efficiency and Renewable Energy.

- Worrell, E., N. Martin, and L. Price. 1999. Energy Efficiency and Carbon Dioxide Emission Reduction Opportunities in the U.S. Iron and Steel Sector. LBNL-41724. Berkeley, Calif.: Lawrence Berkeley National Laboratory.

- Brownell, F. William and Matthew F. Goldberg, "Protecting the public's health: The Supreme Court speaks”, EM, Vol. 7, June, pp. 16-19, 2001.

- Kashmanian, Richard M., Mahesh K. Podar, Mark A. Luttner, and Robert G. Graff, "The use and impact of intraplant trading in the iron and steel industry to reduce water pollution", The Environmental Professional, Vol. 17, December, pp. 309-315, 1995.

- Kerr, Greiner, Andersen, and April, Inc., “Analysis and Evaluation of the EPA Common Sense Initiative”, Washington, D.C.: Prepared for the U.S. EPA, 1999.

- Ontario Minstry of Labour, http://www.gov.on.ca/lab/ohs/radioe.htm, Last accessed July 26, 2002. 
- Podar, Mahesh and Richard M. Kashmanian, "Charting a new course”, Forum for Applied Research and Public Policy, Vol. 13, Fall, pp. 40-44, 1998.

- U.S. Environmental Protection Agency (EPA), "Profile of the Iron and Steel Industry: EPA Office of Compliance Sector Notebook Project”, Washington, D.C.: EPA/310-R-95-005, 1995.

- U.S. Environmental Protection Agency (EPA), "Development Document for Proposed Effluent Limitations Guidelines and Standards for the Iron and Steel Manufacturing Point Source Category”, Washington, D.C.: EPA Office of Water, EPA-821-B-00-011, 2000.

- U.S. General Accounting Office (GAO), "EPA's Common Sense Initiative GAO//RCED”, 97-164, Washington, D.C.: U.S. General Accounting Office, 1997.

- Wrona, Leonard M and Gillian Julien, "Pollution prevention in the steel industry - toward a zero waste plant”, Iron and Steel Engineer, 1997. 


\section{APPENDIX A}

\section{Design for Experiment of Direct Microwave Steel-making (Phase 2)-1}

Iron ore type: Magnetite

May 2002

\begin{tabular}{|c|c|c|c|c|c|c|c|c|}
\hline \multirow[t]{2}{*}{ Sample No. } & \multirow{2}{*}{$\begin{array}{l}\text { Iron } \\
\text { ore \% }\end{array}$} & \multirow{2}{*}{$\begin{array}{l}\text { Coal } \\
(\%)\end{array}$} & \multirow{2}{*}{$\begin{array}{l}\text { Lime } \\
(\%)\end{array}$} & \multicolumn{2}{|c|}{ Binder ratio } & \multirow{2}{*}{$\begin{array}{c}\text { Compact } \\
\text { State }\end{array}$} & \multirow{2}{*}{$\begin{array}{l}\text { Time } \\
\text { (min) }\end{array}$} & \multirow{2}{*}{$\begin{array}{l}\text { KW \& } \\
C^{0}\end{array}$} \\
\hline & & & & Type & $\%$ & & & \\
\hline \multicolumn{9}{|c|}{ a. Change the Ratio of Iron Ore and Coal, Using Bentonite as Binder } \\
\hline M80-C15-L5-B2 & 80 & 15 & \multirow{3}{*}{5} & \multirow{3}{*}{$\begin{array}{l}\text { Bent- } \\
\text { onite }\end{array}$} & \multirow{3}{*}{2} & \multirow{3}{*}{$\begin{array}{l}\text { Tight } \\
\text { (3000 psi) }\end{array}$} & \multirow{3}{*}{7} & \multirow[t]{3}{*}{1.6} \\
\hline M75-C20-L5-B2 & 75 & 20 & & & & & & \\
\hline M70-C25-L5-B2 & 70 & 25 & & & & & & \\
\hline \multicolumn{9}{|c|}{ b. Change the Ratio of Iron Ore and Coal, Using Starch as Binder } \\
\hline M80-C15-L5-S2 & 80 & 15 & \multirow{3}{*}{5} & \multirow{3}{*}{ Starch } & \multirow{3}{*}{2} & \multirow{3}{*}{$\begin{array}{l}\text { Tight } \\
\text { (3000 psi) }\end{array}$} & \multirow{3}{*}{7} & \multirow[t]{3}{*}{1.6} \\
\hline M75-C20-L5-S2 & 75 & 20 & & & & & & \\
\hline M70-C25-L5-S2 & 70 & 25 & & & & & & \\
\hline \multicolumn{9}{|c|}{ c. Change the Compact States 1 with half pressure } \\
\hline M80-C15-L5-S2-O1 & 80 & 15 & \multirow{3}{*}{5} & \multirow{3}{*}{ Starch } & \multirow{3}{*}{2} & \multirow{3}{*}{$\begin{array}{l}\text { Loose } 1 \\
\text { (1500 psi) }\end{array}$} & \multirow{3}{*}{7} & \multirow[t]{3}{*}{1.6} \\
\hline M75-C20-L5-S2-O1 & 75 & 20 & & & & & & \\
\hline M70-C25-L5-S2-O1 & 70 & 25 & & & & & & \\
\hline \multicolumn{9}{|c|}{ d. Change the Compact States 2 without pressure } \\
\hline M80-C15-L5-S0-O0 & 80 & 15 & \multirow{3}{*}{5} & \multirow{3}{*}{ Non } & & & & 1.6 \\
\hline M75-C20-L5-S0-O0 & 75 & 20 & & & 0 & Loose2 & 7 & \\
\hline M70-C25-L5-S0-O0 & 70 & 25 & & & & (Ground) & & \\
\hline e. Change the Heati & ig Powe & (Temp & rature) & & & & & \\
\hline M75-C20-L5-S2-P1.0 & & & & & & & & 1.0 \\
\hline M75-C20-L5-S2-P1.3 & 75 & 20 & 5 & Starch & 2 & Tight & 7 & 1.3 \\
\hline M75-C20-L5-S2-P1.9 & & & & & & (3000 psi) & & 1.9 \\
\hline f. Change the Heatil & g Time & & & & & & & \\
\hline M75-C20-L5-S2-T3 & & & & & & & 3 & 1.6 \\
\hline M75-C20-L5-S2-T5 & 75 & 20 & 5 & Starch & 2 & Tight & 5 & \\
\hline M75-C20-L5-S2-T9 & & & & & & (3000 psi) & 9 & \\
\hline
\end{tabular}




\begin{tabular}{|c|c|c|c|c|c|c|c|c|}
\hline \multicolumn{9}{|c|}{ Continued from “Design for Experiment of Direct Microwave Steel-making (Phase 2)-1” } \\
\hline \multirow{2}{*}{\multicolumn{9}{|c|}{\begin{tabular}{l|l} 
Sample No. & $\begin{array}{l}\text { Iron } \\
\text { ore \% }\end{array}$ \\
\multicolumn{2}{l}{ g. Change the Lime Ratio }
\end{tabular}}} \\
\hline & & & & & & & & \\
\hline M73-C20-L7-S2 & 73 & \multirow{3}{*}{20} & 7 & \multirow{3}{*}{ Starch } & \multirow{3}{*}{2} & \multirow{3}{*}{$\begin{array}{l}\text { Tight } \\
\text { (3000 psi) }\end{array}$} & \multirow{3}{*}{7} & \multirow[t]{3}{*}{1.6} \\
\hline M70-C20-L10-S2 & 70 & & 10 & & & & & \\
\hline M65-C20-L15-S2 & 65 & & 15 & & & & & \\
\hline \multicolumn{9}{|c|}{ h. Change the Reducing Agent: Replace Coal with Coal-S (High S) } \\
\hline M80-Cs15-L5-S2 & 80 & 15 & \multirow{3}{*}{5} & \multirow{3}{*}{ Starch } & \multirow{3}{*}{2} & \multirow{3}{*}{$\begin{array}{l}\text { Tight } \\
\text { (3000 psi) }\end{array}$} & \multirow{3}{*}{7} & \multirow[t]{3}{*}{1.6} \\
\hline M75-Cs20-L5-S2 & 75 & 20 & & & & & & \\
\hline M70-Cs25-L5-S2 & 70 & 25 & & & & & & \\
\hline \multicolumn{9}{|c|}{ i. Change the Reducing Agent: Replace Coal with Graphite } \\
\hline M80-G15-L5-S2 & 80 & 15 & \multirow{3}{*}{5} & \multirow{3}{*}{ Starch } & \multirow{3}{*}{2} & \multirow{3}{*}{$\begin{array}{l}\text { Tight } \\
\text { (3000 psi) }\end{array}$} & \multirow{3}{*}{7} & \multirow[t]{3}{*}{1.6} \\
\hline M75-G20-L5-S2 & 75 & 20 & & & & & & \\
\hline M70-G25-L5-S2 & 70 & 25 & & & & & & \\
\hline \multicolumn{9}{|c|}{ j. Replace Starch with Molasses } \\
\hline M80-C15-L5-m4 & 80 & 15 & \multirow{3}{*}{5} & \multirow{3}{*}{ molasses } & \multirow{3}{*}{4} & \multirow{3}{*}{$\begin{array}{l}\text { Tight } \\
\text { (3000 psi) }\end{array}$} & \multirow{3}{*}{7} & \multirow[t]{3}{*}{1.6} \\
\hline M75-C20-L5-m4 & 75 & 20 & & & & & & \\
\hline M70-C25-L5-m4 & 70 & 25 & & & & & & \\
\hline \multicolumn{9}{|c|}{ k. Change the Ratio of Binders 1} \\
\hline M80-C15-L5-S5 & 80 & 15 & \multirow[b]{2}{*}{5} & \multirow[b]{2}{*}{ Starch } & \multirow[b]{2}{*}{5} & & & 1.6 \\
\hline M70-C25-L5-S5 & 70 & 25 & & & & $\begin{array}{l}\text { Tight } \\
\text { (3000 psi) }\end{array}$ & 7 & \\
\hline l. Change the Comp & act State & 1 of $B$ & der 1 & & & & & \\
\hline M80-C15-L5-S5-O1 & 80 & 15 & & & & & & 1.6 \\
\hline M70-C25-L5-S5-O1 & 70 & 25 & 5 & Starch & 5 & $\begin{array}{l}\text { Loose } 1 \\
\text { (1500 psi) }\end{array}$ & 7 & \\
\hline
\end{tabular}




\section{Sample Preparation for Microwave Steel-making (Phase 2)-1}

Magnetite

June 2002

\begin{tabular}{|c|c|c|c|c|c|c|c|}
\hline \multirow[t]{2}{*}{ Sample } & \multirow[t]{2}{*}{ No. } & \multicolumn{2}{|c|}{ Before Heating (g) } & \multicolumn{4}{|c|}{ After Heating (g) } \\
\hline & & Crucible & Sample & Total wt & Slag & Steel & Yield \% \\
\hline \multicolumn{8}{|c|}{ a. Change the Ratio of Iron Ore and Coal, Using Bentonite as Binder } \\
\hline \multirow{3}{*}{ M80-C15-L5-B2 } & Ma1-1 & 18.26 & 5.60 & 22.01 & & & \\
\hline & Ma1-2 & 18.45 & 5.50 & 22.10 & & 1.69 & \\
\hline & Ma1-3 & 18.79 & 5.59 & 22.49 & 1.98 & 1.72 & 61.15 \\
\hline \multirow{3}{*}{ M75-C20-L5-B2 } & Ma2-1 & 18.26 & 5.42 & 21.42 & & & \\
\hline & Ma2-2 & 18.58 & 5.42 & 21.75 & & 2.33 & \\
\hline & Ma2-3 & 18.3 & 5.29 & 21.35 & .83 & 2.22 & 88.96 \\
\hline \multirow{3}{*}{ M70-C25-L5-B2 } & Ma3-1 & 18.91 & 5.34 & 21.76 & & & \\
\hline & Ma3-2 & 18.11 & 5.08 & 20.87 & & 1.74 & \\
\hline & Ma3-3 & 18.53 & 5.24 & 21.32 & .55 & 2.24 & 97.09 \\
\hline \multicolumn{8}{|c|}{ b. Change the Ratio of Iron Ore and Coal, Using Starch as Binder } \\
\hline \multirow{3}{*}{ M80-C15-L5-S2 } & Mb1-1 & 18.37 & 5.71 & 22.12 & & & \\
\hline & Mb1-2 & 18.07 & 5.66 & 21.79 & & 1.65 & \\
\hline & Mb1-3 & 18.49 & 5.49 & 22.09 & 1.96 & 1.64 & 59.37 \\
\hline \multirow{3}{*}{ M75-C20-L5-S2 } & Mb2-1 & 18.61 & 5.57 & 21.81 & & & \\
\hline & $\mathrm{Mb} 2-2$ & 18.48 & 5.62 & 21.64 & & 2.43 & \\
\hline & Mb2-3 & 18.36 & 5.95 & 21.76 & .85 & 2.55 & 90.85 \\
\hline \multirow{3}{*}{ M70-C25-L5-S2 } & Mb3-1 & 18.28 & 5.18 & 21.09 & & & \\
\hline & Mb3-2 & 18.11 & 5.05 & 20.92 & 0.60 & 2.21 & 99.39 \\
\hline & Mb3-3 & 18.89 & 5.29 & 21.77 & .55 & 2.33 & 100.03 \\
\hline \multicolumn{8}{|c|}{ c. Change the Compact States 1 with half Pressure } \\
\hline \multirow{3}{*}{ M80-C15-L5-S2-O1 } & Mc1-1 & 18.34 & 5.41 & 21.85 & & & \\
\hline & Mc1-2 & 18.47 & 5.64 & 22.20 & & 1.68 & \\
\hline & Mc1-3 & 18.62 & 5.56 & 22.31 & 2.08 & 1.61 & 57.55 \\
\hline \multirow{3}{*}{ M75-C20-L5-S2-O1 } & Mc2-1 & 18.53 & 5.22 & 21.62 & & & \\
\hline & Mc2-2 & 18.47 & 5.41 & 21.69 & & 2.10 & \\
\hline & Mc2-3 & 18.40 & 5.26 & 21.47 & .90 & 2.17 & 87.45 \\
\hline
\end{tabular}




\begin{tabular}{|c|c|c|c|c|c|c|c|}
\hline \multicolumn{8}{|c|}{ Continued from "Sample Preparation for Microwave Steel-making (Phase 2)-1” } \\
\hline \multirow[t]{2}{*}{ Sample } & \multirow[t]{2}{*}{ No. } & \multicolumn{2}{|c|}{ Before Heating (g) } & \multicolumn{4}{|c|}{ After Heating (g) } \\
\hline & & Crucible & Sample & Total wt & Slag & Steel & Yield \% \\
\hline \multirow{3}{*}{ M70-C25-L5-S2-O1 } & Mc3-1 & 18.55 & 5.15 & 21.38 & & & \\
\hline & Mc3-2 & 18.31 & 4.73 & 21.96 & & 2.10 & \\
\hline & Mc3-3 & 18.24 & 5.17 & 21.06 & .55 & 2.27 & 99.72 \\
\hline \multicolumn{8}{|c|}{ d. Change the Compact States 2 without Press } \\
\hline \multirow{3}{*}{ M80-C15-L5-S0-O0 } & Md1-1 & 18.23 & 6.69 & 22.12 & & & \\
\hline & Md1-2 & 18.26 & 5.97 & 22.20 & & 1.44 & \\
\hline & Md1-3 & 24.66 & 5.72 & 28.71 & 2.92 & 1.13 & 39.26 \\
\hline \multirow{3}{*}{ M75-C20-L5-S0-O0 } & Md2-1 & 24.41 & 5.44 & 27.6 & & & \\
\hline & Md2-2 & 23.83 & 5.58 & 26.63 & & 1.75 & \\
\hline & Md2-3 & 23.59 & 5.41 & 26.56 & .93 & 2.04 & 79.93 \\
\hline \multirow{3}{*}{ M70-C25-L5-S0-O0 } & Md3-1 & 23.60 & 5.76 & 26.36 & & & \\
\hline & Md3-2 & 24.63 & 5.30 & 26.74 & & 1.28 & \\
\hline & Md3-3 & 23.01 & 5.67 & 25.57 & .82 & 1.74 & 69.70 \\
\hline \multicolumn{8}{|c|}{ e. Change the Heating Power } \\
\hline \multirow{3}{*}{ M75-C20-L5-S2-P1.0 } & Me1-1 & 18.05 & 5.11 & 20.97 & & & \\
\hline & Me1-2 & 18.17 & 5.20 & 21.2 & & & \\
\hline & Me1-3 & & & & & & LOOSE \\
\hline \multirow{3}{*}{ M75-C20-L5-S2-P1.3 } & Me2-1 & 18.77 & 5.50 & 21.92 & & & \\
\hline & Me2-2 & 18.67 & 5.60 & 21.87 & & 2.44 & \\
\hline & Me2-3 & 18.14 & 5.66 & 21.37 & .77 & 2.46 & 92.13 \\
\hline \multirow{3}{*}{ M75-C20-L5-S2-P1.9 } & Me3-1 & 18.54 & 5.72 & 21.74 & & & \\
\hline & Me3-2 & 18.16 & 5.74 & 21.38 & & 2.53 & \\
\hline & Me3-3 & 18.83 & 5.39 & 21.86 & .66 & 2.37 & 93.21 \\
\hline \multicolumn{8}{|c|}{ f. Change the Heating Time } \\
\hline \multirow{3}{*}{ M75-C20-L5-S2-T3 } & Mf1-1 & 18.57 & 5.77 & 21.85 & & & \\
\hline & Mf1-2 & 18.07 & 5.76 & 21.40 & & 2.54 & \\
\hline & Mf1-3 & 18.77 & 6.05 & 22.23 & .84 & 2.62 & 91.8 \\
\hline \multirow{3}{*}{ M75-C20-L5-S2-T5 } & Mf2-1 & 18.54 & 5.72 & 21.81 & & & \\
\hline & Mf2-2 & 18.74 & 5.96 & 22.14 & & 2.57 & \\
\hline & Mf2-3 & 18.72 & 5.77 & 22.02 & .79 & 2.51 & 92.21 \\
\hline
\end{tabular}




\begin{tabular}{|c|c|c|c|c|c|c|c|}
\hline \multicolumn{8}{|c|}{ Continued from "Sample Preparation for Microwave Steel-making (Phase 2)-1” } \\
\hline \multirow[t]{2}{*}{ Sample } & \multirow[t]{2}{*}{ No. } & \multicolumn{2}{|c|}{ Before Heating (g) } & \multicolumn{4}{|c|}{ After Heating (g) } \\
\hline & & Crucible & Sample & Total wt & Slag & Steel & Yield \% \\
\hline \multirow{3}{*}{ M75-C20-L5-S2-T9 } & Mf3-1 & 18.22 & 5.63 & 21.44 & & & \\
\hline & Mf3-2 & 18.80 & 5.55 & 22.00 & & 2.40 & \\
\hline & Mf3-3 & 18.86 & 5.70 & 22.13 & .78 & 2.49 & 92.60 \\
\hline \multicolumn{8}{|c|}{ g. Change the Lime Ratio } \\
\hline \multirow{3}{*}{ M73-C20-L7-S2 } & Mg1-1 & 18.48 & 5.59 & 21.70 & & & \\
\hline & Mg1-2 & 18.21 & 5.55 & 21.36 & & 2.36 & \\
\hline & Mg1-3 & 18.58 & 5.51 & 21.73 & .83 & 2.32 & 91.7 \\
\hline \multirow{3}{*}{ M70-C20-L10-S2 } & Mg2-1 & 17.89 & 5.21 & 20.83 & & & \\
\hline & Mg2-2 & 18.53 & 5.26 & 21.51 & & 2.14 & \\
\hline & Mg2-3 & 17.85 & 5.15 & 20.78 & .85 & 2.08 & 91.73 \\
\hline \multirow{3}{*}{ M65-C20-L15-S2 } & Mg3-1 & 18.51 & 5.05 & 21.37 & & & \\
\hline & Mg3-2 & 18.44 & 5.41 & 21.49 & & 2.12 & \\
\hline & Mg3-3 & 18.02 & 5.19 & 20.95 & .89 & 2.04 & 96.14 \\
\hline \multicolumn{8}{|c|}{ h. Change the Reducing Agent: Replace Coal with Coal-S (High S) } \\
\hline \multirow{3}{*}{ M80-Cs15-L5-S2 } & Mh1-1 & 18.24 & 6.24 & 22.63 & & & \\
\hline & Mh1-2 & 18.42 & 5.94 & 22.55 & & 1.08 & \\
\hline & Mh1-3 & 18.56 & 5.93 & 22.66 & 3.00 & 1.10 & 36.86 \\
\hline \multirow{3}{*}{ M75-Cs20-L5-S2 } & Mh2-1 & 18.26 & 5.37 & 21.63 & & & \\
\hline & Mh2-2 & 18.14 & 5.54 & 21.66 & & 1.64 & \\
\hline & Mh2-3 & 18.51 & 5.6 & 22.05 & 1.81 & 1.73 & 65.49 \\
\hline \multirow{3}{*}{ M70-Cs25-L5-S2 } & Mh3-1 & 18.72 & 4.94 & 21.51 & & & \\
\hline & Mh3-2 & 18.42 & 5.06 & 21.28 & & 2.11 & \\
\hline & Mh3-3 & 18.41 & 5.38 & 21.45 & .84 & 2.20 & 92.87 \\
\hline \multicolumn{8}{|c|}{ i. Change the Reducing Agent: Replace Coal with Graphite } \\
\hline \multirow{3}{*}{ M81-G14-L5-S2 } & Mi0-1 & 18.44 & 5.00 & 21.41 & 0.52 & 2.43 & 95.4 \\
\hline & Mi0-2 & 18.33 & 5.00 & 21.28 & 0.52 & 2.43 & 95.4 \\
\hline & Mi0-3 & 18.19 & 5.61 & 21.48 & 0.71 & 2.58 & 90.3 \\
\hline \multirow{3}{*}{ M80-G15-L5-S2 } & Mi1-1 & 17.61 & 5.25 & 20.69 & & & \\
\hline & Mi1-2 & 18.30 & 5.35 & 21.43 & & 2.50 & \\
\hline & Mi1-3 & 18.77 & 5.19 & 21.81 & .56 & 2.48 & 94.96 \\
\hline
\end{tabular}




\begin{tabular}{|c|c|c|c|c|c|c|c|}
\hline \multicolumn{8}{|c|}{ Continued from "Sample Preparation for Microwave Steel-making (Phase 2)-1” } \\
\hline \multirow[t]{2}{*}{ Sample } & \multirow[t]{2}{*}{ No. } & \multicolumn{2}{|c|}{ Before Heating (g) } & \multicolumn{4}{|c|}{ After Heating (g) } \\
\hline & & Crucible & Sample & Total wt & Slag & Steel & Yield \% \\
\hline \multirow{3}{*}{ M75-G20-L5-S2 } & Mi2-1 & 18.61 & 4.92 & 21.49 & & & \\
\hline & Mi2-2 & 18.78 & 4.76 & 21.55 & & & \\
\hline & Mi2-3 & 18.49 & 4.68 & 21.18 & & & LOOSE \\
\hline \multirow{3}{*}{ M70-G25-L5-S2 } & Mi3-1 & 18.71 & 4.44 & 21.08 & & & \\
\hline & Mi3-2 & 18.74 & 4.54 & 21.45 & & & \\
\hline & Mi3-3 & 18.31 & 4.49 & 20.86 & & & LOOSE \\
\hline \multicolumn{8}{|c|}{ j. Replace Starch with Molasses } \\
\hline \multirow{3}{*}{ M80-C15-L5-m4 } & Mj1-1 & 18.57 & 6.22 & 22.48 & & & \\
\hline & Mj1-2 & 18.82 & 5.54 & 22.31 & & 1.87 & \\
\hline & Mj1-3 & 17.80 & 6.01 & 21.59 & 1.72 & 2.07 & 68.45 \\
\hline \multirow{3}{*}{ M75-C20-L5-m4 } & Mj2-1 & 18.38 & 5.66 & 21.60 & & & \\
\hline & Mj2-2 & 18.29 & 5.55 & 21.47 & & 2.46 & \\
\hline & $\mathrm{Mj} 2-3$ & 18.48 & 5.74 & 21.73 & .75 & 2.5 & 92.32 \\
\hline \multirow{3}{*}{ M70-C25-L5-m4 } & Mj3-1 & 18.29 & 5.42 & 21.17 & & & \\
\hline & Mj3-2 & 18.36 & 5.29 & 21.36 & & 1.58 & \\
\hline & Mj3-3 & 18.60 & 5.37 & 21.43 & .62 & 2.21 & 93.47 \\
\hline \multicolumn{8}{|c|}{ k. Change the Ratio of Binders 1: Starch } \\
\hline \multirow{3}{*}{ M80-C15-L5-S5 } & Mk1-1 & 19.15 & 5.63 & 22.76 & & & \\
\hline & Mk1-2 & 18.09 & 5.39 & 21.57 & & 1.38 & \\
\hline & Mk1-3 & 18.12 & 5.64 & 21.75 & 2.06 & 1.57 & 55.32 \\
\hline \multirow{3}{*}{ M70-C25-L5-S5 } & Mk3-1 & 18.47 & 5.16 & 20.91 & & & \\
\hline & Mk3-2 & 18.34 & 5.17 & 21.01 & & 2.22 & \\
\hline & Mk3-3 & 18.29 & 4.84 & 21.27 & .96 & 2.02 & 94.79 \\
\hline \multicolumn{8}{|c|}{ l. Change the Compact States 1 of Binder 1: Starch } \\
\hline \multirow{3}{*}{ M80-C15-L5-S5-O1 } & Ml1-1 & 18.53 & 5.24 & 21.76 & & & \\
\hline & Ml1-2 & 18.97 & 5.63 & 22.48 & & 1.76 & \\
\hline & Ml1-3 & 18.88 & 5.44 & 22.27 & 1.67 & 1.72 & 62.83 \\
\hline \multirow{3}{*}{ M70-C25-L5-S5-O1 } & Ml3-1 & 18.3 & 4.91 & 20.8 & & & \\
\hline & Ml3-2 & 18.44 & 4.99 & 21.04 & & 2.11 & \\
\hline & Ml3-3 & 18.63 & 5.04 & 21.19 & .40 & 2.16 & 97.34 \\
\hline
\end{tabular}




\begin{tabular}{|c|c|c|c|c|c|c|c|}
\hline \multicolumn{8}{|c|}{ Continued from "Sample Preparation for Microwave Steel-making (Phase 2)-1" } \\
\hline \multirow[t]{2}{*}{ Sample } & \multirow[t]{2}{*}{ No. } & \multicolumn{2}{|c|}{ Before Heating (g) } & \multicolumn{4}{|c|}{ After Heating (g) } \\
\hline & & Crucible & Sample & Total wt & Slag & Steel & Yield \% \\
\hline \multicolumn{8}{|c|}{ b. Change the Ratio of Iron Ore and Coal, Using Starch as Binder } \\
\hline \multirow{3}{*}{ M80-C15-L5-S2 } & Mb1-1 & 18.37 & 5.71 & 22.12 & & & \\
\hline & Mb1-2 & 18.07 & 5.66 & 21.79 & & 1.65 & \\
\hline & Mb1-3 & 18.49 & 5.49 & 22.09 & 1.96 & 1.64 & 59.37 \\
\hline \multirow{3}{*}{ M75-C20-L5-S2 } & Mb2-1 & 18.61 & 5.57 & 21.81 & & & \\
\hline & Mb2-2 & 18.48 & 5.62 & 21.64 & & 2.43 & \\
\hline & Mb2-3 & 18.36 & 5.95 & 21.76 & .85 & 2.55 & 90.85 \\
\hline \multirow{3}{*}{ M72-C23-L5-S2 } & Mb4-1 & 17.98 & 5.15 & 20.90 & & & \\
\hline & Mb4-2 & 18.72 & 4.99 & 21.53 & & 2.19 & \\
\hline & Mb4-3 & 18.16 & 5.25 & 21.12 & & 2.29 & 96.31 \\
\hline \multirow{3}{*}{ M71-C24-L5-S2 } & Mb5-1 & 18.78 & 5.29 & 21.66 & & & \\
\hline & Mb5-2 & 18.55 & 5.30 & 21.33 & & 2.32 & \\
\hline & Mb5-3 & 18.27 & 5.27 & 21.16 & & 2.26 & 96.03 \\
\hline \multirow{3}{*}{ M70-C25-L5-S2 } & Mb3-1 & 18.28 & 5.18 & 21.09 & & & \\
\hline & Mb3-2 & 18.11 & 5.05 & 20.92 & 0.60 & 2.21 & 99.39 \\
\hline & Mb3-3 & 18.89 & 5.29 & 21.77 & .55 & 2.33 & 100.03 \\
\hline \multirow{3}{*}{ M69-C26-L5-S2 } & Mb6-1 & 18.69 & 5.28 & 21.54 & & & \\
\hline & Mb6-2 & 18.62 & 5.32 & 21.61 & & 1.83 & 79.26 \\
\hline & Mb6-3 & 18.70 & 5.29 & 21.64 & & 1.82 & 79.27 \\
\hline \multirow{3}{*}{ M68-C27-L5-S2 } & Mb7-1 & 18.44 & 4.93 & 21.14 & & 1.66 & 78.73 \\
\hline & Mb7-2 & & & & & & \\
\hline & Mb7-3 & & & & & & \\
\hline \multirow{3}{*}{ M65-C30-L5-S2 } & Mb8-1 & 18.43 & 4.91 & 21.19 & & 0.95 & 47.32 \\
\hline & Mb8-2 & & & & & & \\
\hline & Mb8-3 & & & & & & \\
\hline \multirow{3}{*}{ M62-C33-L5-S2 } & Mb9-1 & 18.00 & 4.73 & 20.51 & & & loose \\
\hline & Mb9-2 & & & & & & \\
\hline & Mb9-3 & & & & & & \\
\hline
\end{tabular}


h. Change the Reducing Agent: Replace Coal with Coal-S (High S)

\begin{tabular}{|c|c|c|c|c|c|c|c|}
\hline \multirow{3}{*}{ M80-Cs15-L5-S2 } & Mh1-1 & 18.24 & 6.24 & 22.63 & & & \\
\hline & Mh1-2 & 18.42 & 5.94 & 22.55 & & & \\
\hline & Mh1-3 & 18.56 & 5.93 & 22.66 & 3.00 & 1.10 & 36.86 \\
\hline \multirow{3}{*}{ M75-Cs20-L5-S2 } & Mh2-1 & 18.26 & 5.37 & 21.63 & & & \\
\hline & Mh2-2 & 18.14 & 5.54 & 21.66 & & & \\
\hline & Mh2-3 & 18.51 & 5.6 & 22.05 & 1.81 & 1.73 & 65.49 \\
\hline \multirow{3}{*}{ M70-Cs25-L5-S2 } & Mh3-1 & 18.72 & 4.94 & 21.51 & & & \\
\hline & Mh3-2 & 18.42 & 5.06 & 21.28 & & & \\
\hline & Mh3-3 & 18.41 & 5.38 & 21.45 & .84 & 2.20 & 92.87 \\
\hline \multirow{3}{*}{ M68-Cs27-L5-S2 } & Mh4-1 & 18.45 & 5.23 & 21.10 & & & \\
\hline & Mh4-2 & 18.42 & 5.19 & 21.13 & & 2.21 & \\
\hline & Mh4-3 & 18.47 & 5.22 & 21.17 & & 2.20 & 98.54 \\
\hline \multirow{3}{*}{ M65-Cs30-L5-S2 } & Mh5-1 & 18.46 & 5.06 & 21.08 & & & \\
\hline & Mh5-2 & 18.51 & 4.84 & 20.93 & & 1.91 & \\
\hline & Mh5-3 & 18.59 & 4.92 & 21.11 & & 1.93 & 95.95 \\
\hline \multirow{3}{*}{ M62-Cs33-L5-S2 } & Mh6-1 & 18.10 & 4.91 & 20.68 & & & \\
\hline & Mh6-2 & 18.91 & 5.00 & 21.37 & & & \\
\hline & Mh6-3 & 18.34 & 4.71 & 20.78 & & 1.50 & 81.66 \\
\hline \multicolumn{8}{|c|}{ i. Change the Reducing Agent: Replace Coal with Graphite } \\
\hline \multirow{3}{*}{ M81-G14-L5-S2 } & Mi0-1 & 18.44 & 5.00 & 21.41 & 0.52 & 2.43 & 95.4 \\
\hline & Mi0-2 & 18.33 & 5.00 & 21.28 & 0.52 & 2.43 & 95.4 \\
\hline & Mi0-3 & 18.19 & 5.61 & 21.48 & 0.71 & 2.58 & 90.3 \\
\hline \multirow{3}{*}{ M80-G15-L5-S2 } & Mi1-1 & 17.61 & 5.25 & 20.69 & & & \\
\hline & Mi1-2 & 18.30 & 5.35 & 21.43 & & & \\
\hline & Mi1-3 & 18.77 & 5.19 & 21.81 & .56 & 2.48 & 94.96 \\
\hline \multirow{3}{*}{ M79-G16-L5-S2 } & Mi4-1 & 18.25 & 5.11 & 21.24 & & & loose \\
\hline & Mi4-2 & 18.52 & 5.00 & 21.46 & & & loose \\
\hline & Mi4-3 & & & & & & \\
\hline \multirow[b]{2}{*}{ M78-G17-L5-S2 } & Mi5-1 & 18.02 & 4.90 & 20.89 & & & loose \\
\hline & Mi5-2 & 18.98 & 5.01 & 21.86 & & & loose \\
\hline
\end{tabular}




\begin{tabular}{|l|l|l|l|l|l|l|l|}
\hline & Mi5-3 & 18.67 & 5.01 & 21.61 & & 1.66 & loose \\
\hline \multirow{3}{*}{ M77-G18-L5-S2 } & Mi6-1 & 18.54 & 4.94 & 21.40 & & & loose \\
\cline { 2 - 9 } & Mi6-2 & 18.08 & 4.62 & 20.76 & & & loose \\
\cline { 2 - 9 } & Mi6-3 & & & & & & \\
\hline \multirow{3}{*}{ M76-G19-L5-S2 } & Mi7-1 & 18.77 & 4.81 & 21.56 & & & loose \\
\cline { 2 - 9 } & Mi7-2 & 18.16 & 4.87 & 20.90 & & & loose \\
\cline { 2 - 9 } & Mi7-3 & & & & & & \\
\hline
\end{tabular}




\section{Design for Experiment of Direct Microwave Steel-making (Phase 2)-H}

Iron ore type: Hematite

July 2002

\begin{tabular}{|c|c|c|c|c|c|c|c|c|}
\hline \multirow[t]{2}{*}{ Sample No. } & \multirow{2}{*}{$\begin{array}{l}\text { Iron } \\
\text { ore } \%\end{array}$} & \multirow{2}{*}{$\begin{array}{l}\text { Coal } \\
(\%)\end{array}$} & \multirow{2}{*}{$\begin{array}{l}\text { Lime } \\
(\%)\end{array}$} & \multicolumn{2}{|c|}{ Binder ratio } & \multirow{2}{*}{$\begin{array}{c}\text { Compact } \\
\text { State }\end{array}$} & \multirow{2}{*}{$\begin{array}{l}\text { Time } \\
\text { (min) }\end{array}$} & \multirow{2}{*}{$\begin{array}{l}\text { KW \& } \\
C^{0}\end{array}$} \\
\hline & & & & Type & $\%$ & & & \\
\hline \multicolumn{9}{|c|}{ a. Change the Ratio of Iron Ore and Coal, Using Bentonite as Binder } \\
\hline H-C20-L5-B2 & 75 & 20 & \multirow{3}{*}{5} & \multirow{3}{*}{$\begin{array}{l}\text { Bent- } \\
\text { onite }\end{array}$} & \multirow{3}{*}{2} & \multirow{3}{*}{$\begin{array}{l}\text { Tight } \\
\text { (3000 psi) }\end{array}$} & \multirow{3}{*}{7} & \multirow{3}{*}{1.6} \\
\hline H-C23-L5-B2 & 72 & 23 & & & & & & \\
\hline H-C24.5-L5-B2 & 70.5 & 24.5 & & & & & & \\
\hline \multicolumn{9}{|c|}{ b. Change the Ratio of Iron Ore and Coal, Using Molasses as Binder } \\
\hline H-C20-L5-m4 & 75 & 20 & \multirow{3}{*}{5} & \multirow{3}{*}{$\begin{array}{l}\text { Mola- } \\
\text { sses }\end{array}$} & \multirow{3}{*}{2} & \multirow{3}{*}{$\begin{array}{l}\text { Tight } \\
\text { (3000 psi) }\end{array}$} & \multirow{3}{*}{7} & \multirow{3}{*}{1.6} \\
\hline H-C23-L5-m4 & 72 & 23 & & & & & & \\
\hline H-C24.5-L5-m4 & 70.5 & 24.5 & & & & & & \\
\hline \multicolumn{9}{|c|}{ c. Change the Ratio of Iron Ore and Coal, Using Starch as Binder } \\
\hline H-C20-L5-S2 & 75 & 20 & \multirow{6}{*}{5} & \multirow{6}{*}{ Starch } & \multirow{6}{*}{2} & \multirow{6}{*}{$\begin{array}{l}\text { Tight } \\
\text { (3000 psi) }\end{array}$} & \multirow{6}{*}{7} & \multirow{6}{*}{1.6} \\
\hline H-C23-L5-S2 & 72 & 23 & & & & & & \\
\hline H-C24-L5-S2 & 71 & 24 & & & & & & \\
\hline H-C24.5-L5-S2 & 70.5 & 24.5 & & & & & & \\
\hline H-C25-L5-S2 & 70 & 25 & & & & & & \\
\hline H-C27-L5-S2 & 68 & 27 & & & & & & \\
\hline \multicolumn{9}{|c|}{ d. Change the Compact States 1 with half pressure } \\
\hline H-C20-L5-S2-O1 & 75 & 20 & \multirow{3}{*}{5} & \multirow{3}{*}{ Starch } & & & & 1.6 \\
\hline H-C23-L5-S2-O1 & 72 & 23 & & & 2 & Loose 1 & 7 & \\
\hline H-C24.5-L5-S2-O1 & 70.5 & 24.5 & & & & (1500 psi) & & \\
\hline e. Change the Heat & ig Powe & (Temp & rature) & & & & & \\
\hline H-C24.5-L5-S2-P1.0 & & & & & & & & 1.0 \\
\hline H-C24.5-L5-S2-P1.3 & 70.5 & 24.5 & 5 & Starch & 2 & Tight & 7 & 1.3 \\
\hline H-C24.5-L5-S2-P1.9 & & & & & & (3000 psi) & & 1.9 \\
\hline
\end{tabular}




\begin{tabular}{|c|c|c|c|c|c|c|c|c|}
\hline \multicolumn{9}{|c|}{ Continued from “Design for Experiment of Direct Microwave Steel-making (Phase 2)-1" } \\
\hline \multirow{2}{*}{\multicolumn{2}{|c|}{\begin{tabular}{l|l} 
Sample No. & $\begin{array}{l}\text { Iron } \\
\text { ore } \%\end{array}$ \\
\end{tabular}}} & \multirow[t]{2}{*}{$\begin{array}{l}\text { Coal } \\
(\%)\end{array}$} & \multirow[t]{2}{*}{$\begin{array}{l}\text { Lime } \\
(\%)\end{array}$} & \multicolumn{2}{|c|}{ Binder ratio } & \multirow{2}{*}{$\begin{array}{c}\text { Compact } \\
\text { State }\end{array}$} & \multirow{2}{*}{$\begin{array}{l}\text { Time } \\
\text { (min) }\end{array}$} & \multirow[t]{2}{*}{$\begin{array}{l}\text { KW \& } \\
C^{0}\end{array}$} \\
\hline & & & & & & & & \\
\hline H-C24.5-L5-S2-T3 & \multirow{3}{*}{70.5} & \multirow{3}{*}{24.5} & \multirow{3}{*}{5} & \multirow{3}{*}{ Starch } & \multirow{3}{*}{2} & \multirow{3}{*}{$\begin{array}{l}\text { Tight } \\
\text { (3000 psi) }\end{array}$} & 3 & \multirow[t]{3}{*}{1.6} \\
\hline H-C24.5-L5-S2-T5 & & & & & & & 5 & \\
\hline H-C24.5-L5-S2-T9 & & & & & & & 9 & \\
\hline \multicolumn{9}{|c|}{ g. Change the Lime Ratio } \\
\hline H-C25.1-L 7-S2 & 71.9 & 25.1 & 3 & \multirow{3}{*}{ Starch } & \multirow{3}{*}{2} & \multirow{3}{*}{$\begin{array}{l}\text { Tight } \\
\text { (3000 psi) }\end{array}$} & \multirow{3}{*}{7} & \multirow[t]{3}{*}{1.6} \\
\hline H-C23.3-L10-S2 & 66.7 & 23.3 & 10 & & & & & \\
\hline H-C22.0-L15-S2 & 63.0 & 22.0 & 15 & & & & & \\
\hline \multicolumn{9}{|c|}{ h. Change the Reducing Agent: Replace Coal with High Sulfur Coal-Cs (Eastern Coal) } \\
\hline H-Cs20-L5-S2 & 75 & 20 & \multirow{4}{*}{5} & \multirow{4}{*}{ Starch } & \multirow{4}{*}{2} & \multirow{4}{*}{$\begin{array}{l}\text { Tight } \\
\text { (3000 psi) }\end{array}$} & \multirow{4}{*}{7} & \multirow{4}{*}{1.6} \\
\hline H-Cs25-L5-S2 & 70 & 25 & & & & & & \\
\hline H-Cs27-L5-S2 & 68 & 27 & & & & & & \\
\hline H-Cs30-L5-S2 & 65 & 30 & & & & & & \\
\hline \multicolumn{9}{|c|}{ i. Change the Reducing Agent: Replace Coal with Graphite } \\
\hline H-G12-L5-S2 & 83 & 12 & \multirow{5}{*}{5} & \multirow{5}{*}{ Starch } & \multirow{5}{*}{2} & \multirow{5}{*}{$\begin{array}{l}\text { Tight } \\
\text { (3000 psi) }\end{array}$} & \multirow{5}{*}{7} & \\
\hline H-G13-L5-S2 & 82 & 13 & & & & & & \\
\hline H-G14-L5-S2 & 81 & 14 & & & & & & 1.6 \\
\hline H-G15-L5-S2 & 80 & 15 & & & & & & \\
\hline H-G16-L5-S2 & 79 & 16 & & & & & & \\
\hline
\end{tabular}




\section{Sample Preparation for Microwave Steel-making (Phase 2)-1}

Hematite

June 2002

\begin{tabular}{|c|c|c|c|c|c|c|c|}
\hline \multirow[t]{2}{*}{ Sample } & \multirow[t]{2}{*}{ No. } & \multicolumn{2}{|c|}{ Before Heating (g) } & \multicolumn{4}{|c|}{ After Heating (g) } \\
\hline & & Crucible & Sample & Total wt & Slag & Steel & Yield \% \\
\hline \multicolumn{8}{|c|}{ a. Change the Ratio of Iron Ore and Coal, Using Bentonite as Binder } \\
\hline \multirow{3}{*}{ H75-C20-L5-B2 } & Ha1-1 & 18.37 & 5.21 & 21.36 & & 2.09 & \\
\hline & Ha1-2 & 18.17 & 5.01 & 20.99 & & 1.92 & \\
\hline & Ha1-3 & 18.64 & 5.28 & 21.62 & 0.85 & 2.13 & 88.5 \\
\hline \multirow{3}{*}{ H72-C23-L5-B2 } & Ha2-1 & 18.33 & 5.13 & 21.15 & & & \\
\hline & Ha2-2 & 18.56 & 4.98 & 21.30 & & 1.89 & \\
\hline & Ha2-3 & 18.41 & 5.15 & 21.22 & 0.95 & 1.86 & 82.5 \\
\hline \multirow{3}{*}{ H70.5-C24.5-L5-B2 } & Ha3-1 & 18.60 & 4.93 & 21.27 & & & \\
\hline & Ha3-2 & 17.94 & 4.79 & 20.51 & & 1.81 & \\
\hline & Ha3-3 & 18.00 & 5.16 & 20.75 & 0.70 & 2.05 & 92.7 \\
\hline \multicolumn{8}{|c|}{ b. Change the Ratio of Iron Ore and Coal, Using Molasses as Binder } \\
\hline \multirow{3}{*}{ H75-C20-L5-m4 } & Hb1-1 & 18.50 & 5.27 & 21.45 & & & \\
\hline & Hb1-2 & 17.96 & 5.26 & 20.88 & & 2.17 & \\
\hline & Hb1-3 & 18.77 & 5.22 & 21.59 & 0.96 & 2.22 & 91.5 \\
\hline \multirow{3}{*}{ H72-C23-L5-m4 } & $\mathrm{Hb} 2-1$ & 18.41 & 4.78 & 20.89 & & & \\
\hline & Hb2-2 & 18.68 & 4.87 & 21.26 & & 2.06 & \\
\hline & Hb2-3 & 18.27 & 5.16 & 20.99 & 0.52 & 2.20 & 95.5 \\
\hline \multirow{3}{*}{ H70.5-C24.5-L5-m4 } & Hb3-1 & 18.73 & 5.21 & 21.43 & & & \\
\hline & Hb3-2 & 18.50 & 4.91 & 21.02 & & 2.07 & \\
\hline & Hb3-3 & 18.47 & 5.12 & 21.05 & 0.42 & 2.16 & 96.5 \\
\hline \multicolumn{8}{|c|}{ c. Change the Ratio of Iron Ore and Coal, Using Starch as Binder } \\
\hline \multirow{3}{*}{ H75-C20-L5-S2 } & Hc1-1 & 18.44 & 5.61 & 21.62 & & & \\
\hline & Hc1-2 & 18.44 & 5.71 & 21.67 & & 2.41 & \\
\hline & Hc1-3 & 18.43 & 5.32 & 21.45 & 0.77 & 2.25 & 92.77 \\
\hline \multirow{3}{*}{ H72-C23-L5-S2 } & Hc2-1 & 18.00 & 5.51 & 20.98 & & & \\
\hline & Hc2-2 & 18.38 & 5.40 & 21.34 & & 2.33 & \\
\hline & Hс2-3 & 18.30 & 5.51 & 21.37 & 0.70 & 2.37 & 98.28 \\
\hline
\end{tabular}


Continued from "Sample Preparation for Microwave Steel-making (Phase 2)-1"

\begin{tabular}{|c|c|c|c|c|c|c|c|}
\hline \multirow{2}{*}{ Sample } & \multirow[t]{2}{*}{ No. } & \multicolumn{2}{|c|}{ Before Heating (g) } & \multicolumn{4}{|c|}{ After Heating (g) } \\
\hline & & Crucible & Sample & Total wt & Slag & Steel & Yield \% \\
\hline \multirow{3}{*}{ H71-C24-L5-S2 } & Hс3-1 & 18.62 & 5.62 & 21.68 & & & \\
\hline & Hс3-2 & 18.31 & 5.58 & 21.36 & & 2.40 & \\
\hline & Hc3-3 & 18.04 & 5.77 & 21.17 & 0.64 & 2.49 & 99.99 \\
\hline \multirow{3}{*}{ H70.5-C24.5-L5-S2 } & Hc4-1 & 17.81 & 4.77 & 20.19 & & & \\
\hline & Hс4-2 & 18.85 & 5.10 & 21.29 & & 2.10 & \\
\hline & Hc4-3 & 18.25 & 5.06 & 20.79 & 0.65 & 1.89 & 87.2 \\
\hline \multirow{3}{*}{ H70-C25-L5-S2 } & Hc5-1 & 18.30 & 5.34 & 21.25 & & & \\
\hline & Hc5-2 & 18.57 & 5.35 & 21.45 & & 2.31 & \\
\hline & Hc5-3 & 18.69 & 5.58 & 21.75 & 0.66 & 2.40 & 101.08 \\
\hline \multirow{3}{*}{ H69-C26-L5-S2 } & Hc6-1 & 18.59 & 5.35 & 21.50 & & & \\
\hline & Hc6-2 & 17.97 & 5.48 & 20.99 & & 2.33 & \\
\hline & Hc6-3 & 18.78 & 5.42 & 21.77 & 0.94 & 2.05 & 91.50 \\
\hline \multirow{3}{*}{ H68-C27-L5-S2 } & Hc7-1 & 18.25 & 4.74 & 20.72 & & & \\
\hline & Hc7-2 & 18.71 & 4.80 & 21.25 & & 2.00 & \\
\hline & Hc7-3 & 18.48 & 4.97 & 21.06 & 0.54 & 2.04 & 99.3 \\
\hline
\end{tabular}

d. Change the Compact States 1 with half Pressure

\begin{tabular}{|l|l|l|l|l|l|l|l|}
\hline \multirow{4}{*}{ H75-C20-L5-S2-O1 } & Hd1-1 & 18.19 & 4.86 & 20.85 & & & \\
\cline { 2 - 9 } & Hd1-2 & 17.59 & 4.78 & 20.24 & & 1.99 & \\
\cline { 2 - 9 } & Hd1-3 & 18.88 & 4.98 & 21.59 & 0.63 & 2.08 & 91.6 \\
\hline \multirow{3}{*}{ H72-C23-L5-S2-O1 } & Hd2-1 & 18.46 & 4.49 & 20.85 & & & \\
\cline { 2 - 9 } & Hd2-2 & 18.19 & 4.73 & 20.72 & & 1.95 & \\
\cline { 2 - 9 } & Hd2-3 & 18.38 & 4.73 & 20.93 & 0.61 & 1.94 & 93.7 \\
\hline \multirow{3}{*}{$\begin{array}{l}\text { H70.5-C24.5-L5-S2- } \\
\text { O1 }\end{array}$} & Hd3-1 & 18.74 & 4.41 & 20.73 & & & \\
\cline { 2 - 9 } & Hd3-2 & 18.82 & 4.65 & & & 1.42 & \\
\cline { 2 - 9 } & Hd3-3 & 18.59 & 4.60 & 20.76 & 0.44 & 1.73 & 87.8 \\
\hline \multirow{2}{*}{ e. }
\end{tabular}

e. Change the Heating Power

\begin{tabular}{|l|l|l|l|l|l|l|l|}
\hline \multirow{3}{*}{$\begin{array}{l}\text { H70.5-C24.5-L5-S2- } \\
\text { P1.0 }\end{array}$} & He1-1 & 18.22 & 4.90 & 20.87 & & & \\
\cline { 2 - 9 } & He1-2 & 18.33 & 5.04 & 21.10 & & 2.00 & \\
\cline { 2 - 9 } & He1-3 & 18.65 & 5.04 & 21.41 & 0.74 & 2.02 & 93.5 \\
\hline
\end{tabular}




\begin{tabular}{|c|c|c|c|c|c|c|c|}
\hline \multicolumn{8}{|c|}{ Continued from "Sample Preparation for Microwave Steel-making (Phase 2)-1” } \\
\hline \multirow[t]{2}{*}{ Sample } & \multirow[t]{2}{*}{ No. } & \multicolumn{2}{|c|}{ Before Heating (g) } & \multicolumn{4}{|c|}{ After Heating (g) } \\
\hline & & Crucible & Sample & Total wt & Slag & Steel & Yield \% \\
\hline \multirow{3}{*}{$\begin{array}{l}\text { H70.5-C24.5-L5-S2- } \\
\text { P1.3 }\end{array}$} & He2-1 & 18.19 & 4.91 & 20.86 & & & \\
\hline & He2-2 & 18.97 & 5.17 & 21.75 & & 2.12 & \\
\hline & He2-3 & 18.97 & 5.06 & 21.66 & 0.68 & 2.01 & 92.7 \\
\hline \multirow{3}{*}{$\begin{array}{l}\text { H70.5-C24.5-L5-S2- } \\
\text { P1.9 }\end{array}$} & He3-1 & 18.29 & 4.88 & 20.61 & & & \\
\hline & Нe3-2 & 18.47 & 4.95 & 20.90 & & 1.91 & \\
\hline & He3-3 & 17.67 & 5.03 & 20.13 & 0.46 & 2.00 & 92.8 \\
\hline \multicolumn{8}{|c|}{ f. Change the Heating Time } \\
\hline \multirow{3}{*}{$\begin{array}{l}\text { H70.5-C24.5-L5-S2- } \\
\text { T3 }\end{array}$} & Hf1-1 & 18.12 & 4.96 & 20.51 & & & \\
\hline & Hf1-2 & 18.74 & 5.03 & 21.23 & & 2.03 & \\
\hline & Hf1-3 & 18.44 & 5.01 & 20.98 & 0.63 & 1.91 & 89.0 \\
\hline \multirow{3}{*}{$\begin{array}{l}\text { H70.5-C24.5-L5-S2- } \\
\text { T5 }\end{array}$} & Hf2-1 & 18.39 & 4.93 & 20.90 & & & \\
\hline & Hf2-2 & 18.20 & 5.10 & 20.85 & & 2.08 & \\
\hline & Hf2-3 & 18.21 & 5.10 & 20.66 & 0.37 & 2.08 & 95.2 \\
\hline \multirow{3}{*}{$\begin{array}{l}\text { H70.5-C24.5-L5-S2- } \\
\text { T9 }\end{array}$} & Hf3-1 & 17.69 & 4.97 & 20.30 & & & \\
\hline & Hf3-2 & 17.82 & 5.05 & 20.36 & & 2.04 & \\
\hline & Hf3-3 & 18.62 & 5.05 & 21.22 & 0.49 & 2.11 & 97.5 \\
\hline \multicolumn{8}{|c|}{ g. Change the Lime Ratio } \\
\hline \multirow{3}{*}{ H71.9-C25.1-L3-S2 } & Hg1-1 & 17.82 & 4.89 & 20.41 & & & \\
\hline & Hg1-2 & 17.94 & 4.91 & 20.53 & & 2.07 & \\
\hline & Hg1-3 & 18.15 & 4.87 & 20.67 & 0.44 & 2.08 & 97.7 \\
\hline \multirow{3}{*}{ H66.7-C23.3-L10-S2 } & Hg2-1 & 18.61 & 4.60 & 21.02 & & & \\
\hline & Hg2-2 & 18.15 & 4.38 & 20.46 & & 1.78 & \\
\hline & Hg2-3 & 19.08 & 4.66 & 21.39 & 0.38 & 1.93 & 102.2 \\
\hline \multirow{3}{*}{ H63-C22-L15-S2 } & Hg3-1 & 18.32 & 4.41 & 20.64 & & & \\
\hline & Hg3-2 & 18.54 & 4.59 & 20.96 & & 1.79 & \\
\hline & Hg3-3 & 18.15 & 4.72 & 20.65 & 0.68 & 1.82 & 100.7 \\
\hline \multicolumn{8}{|c|}{ h. Change the Reducing Agent: Replace Coal with Coal-S (High S) } \\
\hline \multirow{3}{*}{ H75-Cs20-L5-S2 } & Hh1-1 & 18.55 & 4.90 & 21.37 & & & \\
\hline & Hh1-2 & 18.57 & 5.04 & 21.54 & & 1.69 & \\
\hline & Hh1-3 & 18.66 & 4.90 & 21.50 & 1.21 & 1.63 & 73.0 \\
\hline
\end{tabular}




\begin{tabular}{|c|c|c|c|c|c|c|c|}
\hline \multicolumn{8}{|c|}{ Continued from "Sample Preparation for Microwave Steel-making (Phase 2)-1” } \\
\hline \multirow[t]{2}{*}{ Sample } & \multirow[t]{2}{*}{ No. } & \multicolumn{2}{|c|}{ Before Heating (g) } & \multicolumn{4}{|c|}{ After Heating (g) } \\
\hline & & Crucible & Sample & Total wt & Slag & Steel & Yield \% \\
\hline \multirow{3}{*}{ H70-Cs25-L5-S2 } & Hh2-1 & 18.65 & 4.74 & 21.10 & & & \\
\hline & Hh2-2 & 18.51 & 4.63 & 20.96 & & 1.93 & \\
\hline & Hh2-3 & 18.32 & 5.07 & 21.01 & 0.57 & 2.12 & 98.3 \\
\hline \multirow{3}{*}{ H68-Cs27-L5-S2 } & Hh3-1 & 18.50 & 4.77 & 20.97 & & & \\
\hline & Hh3-2 & 18.58 & 4.79 & 21.08 & & 1.96 & \\
\hline & Hh3-3 & 18.56 & 4.89 & 21.09 & 0.51 & 2.02 & 99.9 \\
\hline \multirow{3}{*}{ H65-Cs30-L5-S2 } & Hh4-1 & 18.54 & 4.25 & 20.50 & & & \\
\hline & Hh4-2 & 18.08 & 4.58 & 20.27 & & 1.85 & \\
\hline & Hh4-3 & 18.50 & 4.51 & 20.59 & 0.24 & 1.85 & 103.8 \\
\hline \multicolumn{8}{|c|}{ i. Change the Reducing Agent: Replace Coal with Graphite } \\
\hline \multirow{3}{*}{ H83-G12-L5-S2 } & Hi1-1 & 18.67 & 5.10 & 21.58 & & & \\
\hline & Hi1-2 & 18.57 & 5.05 & 21.44 & & 2.16 & \\
\hline & Hi1-3 & 18.33 & 5.05 & 21.21 & 0.77 & 2.11 & 82.8 \\
\hline \multirow{3}{*}{ H82-G13-L5-S2 } & Hi2-1 & 18.40 & 5.00 & 21.32 & & & \\
\hline & $\mathrm{Hi} 2-2$ & 18.30 & 5.05 & 21.67 & & & \\
\hline & Hi2-3 & 19.02 & 5.04 & 21.94 & 0.64 & 2.28 & 90.76 \\
\hline \multirow{4}{*}{ H81-G14-L5-S2 } & Hi3-1 & 18.28 & 5.05 & 21.21 & & & \\
\hline & Hi3-2 & 18.02 & 5.04 & 20.92 & & & \\
\hline & $\mathrm{Hi3}-3$ & 18.70 & 5.04 & 21.62 & 0.64 & 2.28 & 91.88 \\
\hline & $\mathrm{Hi3}-4$ & 17.79 & 5.04 & 20.70 & 0.76 & 2.15 & 86.64 \\
\hline \multirow{3}{*}{ H80-G15-L5-S2 } & Hi1-1 & 18.27 & 5.00 & 21.12 & & & \\
\hline & Hi1-2 & 17.87 & 4.92 & 20.69 & 0.67 & 2.15 & 89.87 \\
\hline & Hi1-3 & 17.87 & 4.92 & 20.65 & 0.54 & 2.25 & 94.05 \\
\hline \multirow{3}{*}{ H79-G16-L5-S2 } & $\mathrm{Hi} 2-1$ & & & & & & \\
\hline & Hi2-2 & & & & & & \\
\hline & Hi2-3 & & & & & & \\
\hline \multicolumn{8}{|c|}{ j. Using Dried Warehouse Coal as Reducing Agent. } \\
\hline \multirow{3}{*}{-Cw24.5-L5-S2 } & Hj3-1 & 17.98 & 5.03 & 21.15 & & & \\
\hline & Hj3-2 & 18.67 & 5.03 & 21.88 & & & \\
\hline & Hj3-3 & 18.50 & 5.00 & 21.65 & 2.19 & 0.96 & 44.8 \\
\hline
\end{tabular}




\section{Design for Experiment of Direct Microwave Steel-making (Phase 2)-H}

Iron ore Hematite

July 2002

\begin{tabular}{|c|c|c|c|c|c|c|c|c|}
\hline \multirow{2}{*}{ Sample No. } & \multirow{2}{*}{$\begin{array}{c}\text { Iron } \\
\text { ore \% }\end{array}$} & \multirow{2}{*}{$\begin{array}{l}\text { Coal } \\
(\%)\end{array}$} & \multirow{2}{*}{$\begin{array}{l}\text { Lime } \\
(\%)\end{array}$} & \multicolumn{2}{|c|}{ Binder ratio } & \multirow{2}{*}{$\begin{array}{c}\text { Compact } \\
\text { State }\end{array}$} & \multirow{2}{*}{$\begin{array}{l}\text { Time } \\
\text { (min) }\end{array}$} & \multirow{2}{*}{$\begin{array}{c}\text { KW \& } \\
C^{0}\end{array}$} \\
\hline & & & & Type & $\%$ & & & \\
\hline \multicolumn{9}{|c|}{ k. Change the Reducing Agent: Western Coal + Graphite } \\
\hline H-C22G1-L5-S2 & 72 & $22+1$ & \multirow{8}{*}{5} & \multirow{8}{*}{ Starch } & \multirow{8}{*}{2} & \multirow{8}{*}{$\begin{array}{l}\text { Tight } \\
\text { (3000 psi) }\end{array}$} & \multirow{8}{*}{7} & \multirow{8}{*}{1.6} \\
\hline H-C19G3-L5-S2 & 73 & $19+3$ & & & & & & \\
\hline H-C16G5-L5-S2 & 74 & $16+5$ & & & & & & \\
\hline H-C10G9-L5-S2 & 76 & $10+9$ & & & & & & \\
\hline H-C8G10-L5-S2 & 77 & $8+10$ & & & & & & \\
\hline H-C6.5G11-L5-S2 & 77.5 & $6.5+11$ & & & & & & \\
\hline H-C5G12-L5-S2 & 78 & $5+12$ & & & & & & \\
\hline H-C3.5G13-L5-S2 & 78.5 & $3.5+13$ & & & & & & \\
\hline \multicolumn{9}{|c|}{ 1. Mix a certain amount of Polyvinyl Alcohol (99-100\% hydrolyzed) in Hc4 + Graphite } \\
\hline H-C24.5-L5-S2-h8 & \multirow{5}{*}{70.5} & 24.5 & \multirow{5}{*}{5} & \multirow{5}{*}{ Starch } & \multirow{5}{*}{2} & \multirow{5}{*}{$\begin{array}{l}\text { Tight } \\
\text { (3000 psi) }\end{array}$} & \multirow{5}{*}{7} & H8 \\
\hline H-C24.5-L5-S2-h4 & & 24.5 & & & & & & \multirow{4}{*}{ H4 } \\
\hline H-C20G3-L5-S2-h4 & & $20+3$ & & & & & & \\
\hline H-C20G2-L5-S2-h4 & & $20+2$ & & & & & & \\
\hline H-C20G1-L5-S2-h4 & & $20+1$ & & & & & & \\
\hline \multicolumn{9}{|c|}{ m. Reducing Agent: Western Coal; Binder: Wheat Flour + Yeast; Pellet: No pressure } \\
\hline H75-C20-L5-W5 & \multirow{4}{*}{75} & \multirow{4}{*}{20} & \multirow{4}{*}{5} & \multirow{4}{*}{$\begin{array}{l}\text { Flour } \\
+ \\
\text { Yeast }\end{array}$} & 5 & \multirow{4}{*}{ Loose } & \multirow{4}{*}{7} & \\
\hline H75-C20-L5-W10 & & & & & 10 & & & \\
\hline H75-C20-L5-W15 & & & & & 15 & & & 1.6 \\
\hline H75-C20-L5-W20 & & & & & 20 & & & \\
\hline n. Reducing Agent: & $\overline{\text { Carbon } \mathrm{f}}$ & om Fly & ash & & & & & \\
\hline H-C20-L5-S2 & 75 & 20 & & & & & & \\
\hline H-C23-L5-S2 & 72 & 23 & & & & & & \\
\hline H-C25-L5-S2 & 70 & 25 & 5 & Starch & 2 & Tight & 7 & 1.6 \\
\hline H-C27-L5-S2 & 68 & 27 & & & & (3000 psi) & & \\
\hline & nr. R & ducing & Agent: $C$ & rbon fro & Fly-a & + Rice & & \\
\hline H-C19-L5-R4.75 & 71.5 & 19 & & & 4.75 & & & \\
\hline
\end{tabular}




\begin{tabular}{|c|c|c|c|c|c|c|c|c|}
\hline H-C18-L5-R9.5 & 67.5 & 18 & \multirow{3}{*}{5} & \multirow{3}{*}{ Rice } & 9.5 & \multirow{3}{*}{$\begin{array}{l}\text { Tight } \\
\text { (3000 psi) }\end{array}$} & \multirow{3}{*}{7} & \multirow{3}{*}{1.6} \\
\hline H-C17-L5-R14.5 & 63.75 & 17 & & & 14.25 & & & \\
\hline H-C16-L5-R19 & 60 & 16 & & & 19 & & & \\
\hline \multicolumn{9}{|c|}{ br. Add 10\% Rice in Mb1 and Mc1 } \\
\hline $\begin{array}{l}\text { M80-C15-L5-S2 } \\
+10 \% \text { Rice }\end{array}$ & 80 & 15 & \multirow[t]{2}{*}{5} & \multirow[t]{2}{*}{$\begin{array}{l}\text { Rice } \\
10 \%\end{array}$} & $\begin{array}{l}\text { Bent } \\
\text { onite } \\
2 \%\end{array}$ & \multirow[t]{2}{*}{$\begin{array}{l}\text { Tight } \\
\text { (3000 psi) }\end{array}$} & \multirow[t]{2}{*}{7} & \multirow[t]{2}{*}{1.6} \\
\hline $\begin{array}{l}\text { M80-C15-L5-S2 } \\
+10 \% \text { Rice }\end{array}$ & 67.5 & 18 & & & $\begin{array}{l}\text { Star } \\
\text { ch } \\
2 \%\end{array}$ & & & \\
\hline
\end{tabular}


Sample Preparation for Microwave Steel-making (Phase 2)-1

Hematite

June 2002

\begin{tabular}{|c|c|c|c|c|c|c|c|}
\hline \multirow{2}{*}{ Sample } & \multirow{2}{*}{ No. } & \multicolumn{2}{|c|}{ Before Heating (g) } & \multicolumn{4}{|c|}{ After Heating (g) } \\
\hline & & Crucible & Sample & Total wt & Slag & Steel & Yield \% \\
\hline \multicolumn{8}{|c|}{ k. Change the Reducing Agent: Western Coal + Graphite } \\
\hline \multirow[b]{2}{*}{ H72-C22G1-L5-S2 } & Hk1-1 & 18.66 & 4.77 & 21.10 & 0.44 & 1.996 & 95.6 \\
\hline & Hk1-2 & 17.88 & 4.30 & 20.16 & 0.44 & 1.837 & 97.6 \\
\hline \multirow[b]{2}{*}{ H73-C19G3-L5-S2 } & Hk2-1 & 18.53 & 4.67 & 20.92 & 0.36 & 2.034 & 98.2 \\
\hline & Hk2-2 & 18.44 & 4.54 & 20.75 & 0.36 & 1.948 & 96.7 \\
\hline \multirow[b]{2}{*}{ H74-C16G5-L5-S2 } & Hk3-1 & 17.95 & 4.49 & 20.29 & 0.38 & 1.963 & 97.2 \\
\hline & Hk3-2 & 18.08 & 4.44 & 20.38 & 0.39 & 1.913 & 95.8 \\
\hline \multirow[b]{2}{*}{ H76-C10G9-L5-S2 } & Hk4-1 & 18.13 & 4.57 & 20.59 & 0.52 & 1.941 & 91.9 \\
\hline & Hk4-2 & 18.98 & 4.44 & 21.41 & 0.68 & 1.752 & 85.4 \\
\hline \multirow[b]{2}{*}{ H77-C8G10-L5-S2 } & Hk5-1 & 18.16 & 4.31 & 20.47 & 0.29 & 2.015 & 99.9 \\
\hline & Hk5-2 & 18.24 & 4.24 & 20.49 & 0.30 & 1.947 & 96.5 \\
\hline \multirow{2}{*}{$\begin{array}{l}\text { H77.5-C6.5G11-L5- } \\
\text { S2 }\end{array}$} & Hk6-1 & 18.39 & 4.38 & 20.72 & 0.37 & 1.961 & 95.0 \\
\hline & Hk6-2 & 18.53 & 4.5 & 20.94 & 0.30 & 2.108 & 99.4 \\
\hline \multirow[b]{2}{*}{ H78-C5G12-L5-S2 } & Hk7-1 & 18.77 & 4.61 & 21.27 & 0.36 & 2.136 & 97.7 \\
\hline & Hk7-2 & 18.82 & 4.54 & 21.29 & 0.32 & 2.147 & 99.7 \\
\hline \multirow{2}{*}{$\begin{array}{l}\text { H78.5-C3.5G13-L5- } \\
\text { S2 }\end{array}$} & Hk8-1 & 18.71 & 4.70 & 21.28 & 0.42 & 2.148 & 95.8 \\
\hline & Hk8-2 & 18.78 & 4.58 & 21.33 & 0.78 & 1.771 & 81.0 \\
\hline \multirow[b]{2}{*}{ H77-C6G7.5-L5-S2 } & Hk9-1 & 18.74 & 5.15 & 21.68 & 0.66 & 2.285 & 90.6 \\
\hline & Hk9-2 & 18.63 & 4.57 & 21.25 & 0.67 & 1.949 & 87.1 \\
\hline \multirow[b]{2}{*}{ H77-C4G5-L5-S2 } & Hk10-1 & 18.86 & 4.73 & 21.98 & 1.86 & 1.260 & 51.9 \\
\hline & Hk10-2 & 18.34 & 5.13 & 21.71 & 1.91 & 1.459 & 55.4 \\
\hline \multirow{2}{*}{$\begin{array}{c}\text { H77-C8G10-L5-S2 } \\
6 \text { min }\end{array}$} & Hk51-1 & 18.36 & 4.26 & 20.68 & 0.42 & 1.899 & 95.2 \\
\hline & Hk51-2 & 18.00 & 4.28 & 20.29 & 0.35 & 1.941 & 96.9 \\
\hline \multirow{2}{*}{$\begin{array}{c}\text { H77-C8G10-L5-S2 } \\
5 \mathrm{~min}\end{array}$} & Hk52-1 & 18.48 & 4.43 & 20.87 & 0.40 & 1.997 & 96.3 \\
\hline & Hk52-2 & 18.45 & 4.43 & 20.82 & 0.33 & 2.038 & 98.3 \\
\hline \multirow{2}{*}{$\begin{array}{c}\text { H77-C8G10-L5-S2 } \\
4 \text { min }\end{array}$} & Hk53-1 & 18.42 & 4.58 & 20.93 & 0.59 & 1.922 & 89.7 \\
\hline & Hk53-2 & 18.12 & 4.46 & 20.59 & 0.64 & 1.830 & 87.7 \\
\hline \multicolumn{8}{|c|}{ l. Mix a certain amount of Polyvinyl Alcohol (99-100\% hydrolyzed) in Hc4 } \\
\hline & Hl1-1 & 19.34 & 4.00 & 20.20 & 0.45 & 1.412 & 88.8 \\
\hline
\end{tabular}




\begin{tabular}{|c|c|c|c|c|c|c|c|}
\hline H-C24.5-L5-S2-h8 & Hl1-2 & 18.76 & 4.16 & 20.63 & 0.61 & 1.261 & 74.9 \\
\hline \multirow[b]{2}{*}{ H-C24.5-L5-S2-h4 } & $\mathrm{Hl} 2-1$ & 18.72 & 4.30 & 20.74 & 0.19 & 1.831 & 103.3 \\
\hline & Hl2-2 & 18.19 & 4.16 & 20.24 & 0.32 & 1.727 & 100.7 \\
\hline \multirow[b]{2}{*}{ H-C20G3-L5-S2-h4 } & Hl3-1 & 18.28 & 4.07 & 20.31 & 0.42 & 1.609 & 94.5 \\
\hline & Hl3-2 & 17.91 & 4.22 & 19.91 & 0.26 & 1.742 & 98.7 \\
\hline \multirow[b]{2}{*}{ H-C20G2-L5-S2-h4 } & $\mathrm{Hl} 4-1$ & 18.36 & 3.94 & 20.23 & 0.46 & 1.414 & 85.0 \\
\hline & $\mathrm{Hl} 4-2$ & 18.59 & 4.10 & 20.62 & 0.36 & 1.672 & 95.9 \\
\hline \multirow[b]{2}{*}{ H-C20G1-L5-S2-h4 } & Hl5-1 & 18.48 & 4.12 & 20.62 & 0.49 & 1.651 & 94.0 \\
\hline & Hl5-2 & 18.25 & 3.90 & 20.26 & 0.46 & 1.550 & 93.2 \\
\hline \multicolumn{8}{|c|}{ gc. Change the Lime Ratio (coated crucible) } \\
\hline \multirow[b]{2}{*}{ H71.9-C25.1-L3-S2 } & Hgc1-1 & 18.47 & 4.75 & 20.78 & 0.33 & 1.979 & 94.9 \\
\hline & Hgc1-2 & 18.51 & 4.54 & 20.60 & 0.20 & 1.885 & 95.0 \\
\hline \multirow[b]{2}{*}{ H70.5-C24.5-L5-S2 } & Hcc4-1 & 18.73 & 4.40 & 20.84 & 0.26 & 1.85 & 98.1 \\
\hline & Hсc4-2 & 18.57 & 4.63 & 20.75 & 0.28 & 1.90 & 95.8 \\
\hline \multirow[b]{2}{*}{ H66.7-C23.3-L10-S2 } & Hgc2-1 & 18.54 & 4.28 & 20.57 & 0.29 & 1.742 & 100.4 \\
\hline & Hgc2-2 & 18.61 & 4.33 & 20.66 & 0.38 & 1.669 & 95.1 \\
\hline \multirow[b]{2}{*}{ H63-C22-L15-S2 } & Hgc3-1 & 18.61 & 4.19 & 20.61 & 0.44 & 1.561 & 97.3 \\
\hline & Hgc3-2 & 18.89 & 4.34 & 21.17 & 0.60 & 1.683 & 101.3 \\
\hline \multicolumn{8}{|c|}{ m. Reducing Agent: Western Coal; Binder: Wheat Flour + Yeast; Pellet: No pressure } \\
\hline \multirow[b]{2}{*}{ H75-C20-L5-W5 } & Hm1-1 & 17.97 & 3.98 & 20.09 & 0.50 & 1.620 & 91.9 \\
\hline & Hm1-2 & 18.90 & 4.11 & 21.09 & 0.56 & 1.633 & 89.7 \\
\hline \multirow[b]{2}{*}{ H75-C20-L5-W10 } & Hm2-1 & 18.21 & 4.01 & 20.25 & 0.44 & 1.600 & 94.4 \\
\hline & Hm2-2 & 18.60 & 3.90 & 20.57 & 0.42 & 1.548 & 93.9 \\
\hline \multirow[b]{2}{*}{ H75-C20-L5-W15 } & Hm3-1 & 18.32 & 3.85 & 20.198 & 0.38 & 1.492 & 95.8 \\
\hline & Hm3-2 & 18.05 & 3.68 & 19.81 & 0.35 & 1.415 & 95.1 \\
\hline \multirow{7}{*}{ H75-C20-L5-W20 } & Hm4-1 & 18.69 & 3.63 & 20.36 & 0.38 & 1.289 & 91.6 \\
\hline & Hm4-2 & 18.56 & 3.72 & 20.30 & 0.32 & 1.416 & 98.2 \\
\hline & $\mathrm{Hm} 4-3$ & 18.19 & 5.33 & 20.63 & 0.39 & 2.049 & 99.2 \\
\hline & $\mathrm{Hm} 4-4$ & 18.41 & 6.00 & 21.03 & 0.36 & 2.263 & 97.3 \\
\hline & $\mathrm{Hm} 4-5$ & 18.63 & 5.86 & & & & \\
\hline & Hm4-6 & 18.28 & 6.66- & & & & \\
\hline & $\mathrm{Hm} 4-7$ & 8.45 & 4.97 & 20.84 & 0.53 & 1.862 & 96.7 \\
\hline H75-C20G1-L5-W5 & Hm5-1 & 18.49 & 6.18 & 21.72 & 0.64 & 2.595 & 95.7 \\
\hline
\end{tabular}




\begin{tabular}{|c|c|c|c|c|c|c|c|}
\hline WHOLE & Hm5-2 & 18.46 & 5.51 & 21.30 & 0.42 & 2.419 & 100.0 \\
\hline \multicolumn{8}{|c|}{ n. Using carbon from fly-ash as reducing agent } \\
\hline \multirow[b]{2}{*}{ H75-C20-L5-S2 } & Hn1-1 & 18.09 & 4.64 & 20.50 & 0.70 & 1.716 & 81.1 \\
\hline & Hn1-2 & 19.19 & 4.56 & 21.60 & 0.67 & 1.737 & 83.6 \\
\hline \multirow[b]{2}{*}{ H72-C23-L5-S2 } & Hn2-1 & 18.33 & 4.27 & 20.28 & 0.66 & 1.161 & 62.1 \\
\hline & Hn2-2 & 18.84 & 4.33 & 21.05 & 0.67 & 1.538 & 81.2 \\
\hline \multirow[b]{2}{*}{ H70-C25-L5-S2 } & Hn3-1 & 18.56 & 4.33 & 20.77 & 0.53 & 1.683 & 91.3 \\
\hline & Hn3-2 & 18.14 & 4.18 & 20.23 & 0.49 & 1.600 & 90.0 \\
\hline \multicolumn{8}{|c|}{ nr. Reducing agent: Fly-ash Carbon + Rice } \\
\hline \multirow[b]{2}{*}{ H71.25 -C19-L5-R4.75 } & Hnr1-1 & 18.20 & 4.015 & 20.08 & 0.51 & 1.370 & 77.2 \\
\hline & Hnr1-2 & 18.32 & 4.055 & 20.26 & 0.64 & 1.296 & 72.3 \\
\hline \multirow{3}{*}{ H67.5-C18-L5-R9.5 } & Hnr2-1 & 18.24 & 3.74 & 20.08 & 0.48 & 1.360 & 86.9 \\
\hline & Hnr2-2 & 18.72 & 3.98 & 20.77 & 0.43 & 1.614 & 96.9 \\
\hline & Hnr2-3 & 18.58 & 4.08 & 20.50 & 0.44 & 1.485 & 87.0 \\
\hline \multirow{2}{*}{$\begin{array}{c}\text { H71.25 -C19-L5-R4.75- } \\
\text { S2 }\end{array}$} & $\begin{array}{l}\text { Hnr1- } \\
\text { 1s }\end{array}$ & 18.72 & 4.21 & 20.92 & 0.52 & 1.677 & 92.0 \\
\hline & $\begin{array}{l}\text { Hnr1- } \\
\text { 2s }\end{array}$ & 18.37 & 4.39 & 20.69 & 0.47 & 1.849 & 97.3 \\
\hline \multirow{2}{*}{$\begin{array}{c}\text { H67.5-C18-L5-R9.5- } \\
\text { S2 }\end{array}$} & $\begin{array}{l}\text { Hnr2- } \\
\text { 1s }\end{array}$ & 18.38 & 3.97 & 20.42 & 0.46 & 1.579 & 96.9 \\
\hline & $\begin{array}{c}\text { Hnr2- } \\
\text { 2s }\end{array}$ & 18.25 & 4.04 & 20.31 & 0.41 & 1.647 & 99.4 \\
\hline \multicolumn{8}{|c|}{ br. Add 10\% Rice in Mb1 and Mc1 under the pellets } \\
\hline \multirow{2}{*}{$\begin{array}{l}\text { M80-C15-L5-S2 } \\
\text { +10\% Rice }\end{array}$} & Mbr1-4 & $18.43+0.5$ & 4.47 & & & 1.71 & 77.5 \\
\hline & Mbr1-5 & $18.52+0.5$ & 4.40 & & & 1.599 & 73.7 \\
\hline \multirow{2}{*}{$\begin{array}{l}\text { M80-C15-L5-S2 } \\
\text { +10\% Rice }\end{array}$} & Mcr1-4 & $18.35+0.5$ & 4.36 & 21.13 & & 1.543 & 71.7 \\
\hline & Mcr1-5 & & & & & & \\
\hline
\end{tabular}


Steel carbon and sulfur analysis results

\begin{tabular}{|c|c|c|}
\hline Sample ID & C\% & S\% \\
\hline Ha1-3 & 0.0763 & 0.522 \\
\hline Ha2-3 & 0.412 & 0.559 \\
\hline Ha3-3 & 2.21 & 0.575 \\
\hline Hb1-3 & 0.577 & 0.484 \\
\hline Hb2-3 & 2.29 & 0.419 \\
\hline Hb3-3 & 2.22 & 0.405 \\
\hline Hc1-3 & 0.0632 & 0.457 \\
\hline Hc2-3 & 1.95 & 0.413 \\
\hline Hc3-3 & 2.31 & 0.467 \\
\hline Hc4-3 & 2.91 & 0.313 \\
\hline Hc5-3 & 2.87 & 0.417 \\
\hline Hc6-3 & 2.77 & 0.328 \\
\hline Hc7-3 & 2.52 & 0.43 \\
\hline Hd1-3 & 0.385 & 0.501 \\
\hline Hd2-3 & 2.44 & 0.498 \\
\hline Hd3-3 & 2.86 & 0.089 \\
\hline
\end{tabular}

Steel carbon and sulfur analysis results

\begin{tabular}{|c|c|c|}
\hline Sample ID & C\% & S\% \\
\hline He1-3 & 2 & 0.499 \\
\hline He2-3 & 2.29 & 0.477 \\
\hline He3-3 & 1.86 & 0.46 \\
\hline Hf1-3 & 2.54 & 0.455 \\
\hline Hf2-3 & 1.83 & 0.494 \\
\hline Hf3-3 & 1.75 & 0.45 \\
\hline Hg1-3 & 2.05 & 0.505 \\
\hline Hg2-3 & 3.01 & 0.263 \\
\hline Hg3-3 & 3.66 & 0.161 \\
\hline Hh1-3 & 0.026 & 1.14 \\
\hline Hh2-3 & 0.804 & 1.45 \\
\hline Hh3-3 & 0.82 & 1.57 \\
\hline Hh4-3 & 0.872 & 1.24 \\
\hline Hi1-3 & 0.0335 & 0.037 \\
\hline Hi2-3 & 0.739 & 0.027 \\
\hline Hi3-3 & 1.03 & 0.029 \\
\hline Hi4-3 & 2.28 & 0.029 \\
\hline
\end{tabular}


Steel sample chemical analysis results

\begin{tabular}{|c|c|c|c|c|c|c|c|c|c|c|c|c|c|}
\hline Sample & Ha1-3 & Ha2-3 & Ha3-3 & Hb1-3 & Hb2-3 & Hb3-3 & Hc1-3 & Hc2-3 & Hc3-3 & Hc4-3 & Hc5-3 & Hc6-3 & Hc7-3 \\
\hline $\mathrm{Cr}$ & $0.075 \%$ & $0.054 \%$ & $0.065 \%$ & $0.060 \%$ & $0.060 \%$ & $0.059 \%$ & $0.061 \%$ & $0.064 \%$ & $0.058 \%$ & $0.061 \%$ & $0.068 \%$ & $0.062 \%$ & $0.063 \%$ \\
\hline $\mathbf{P}$ & $0.105 \%$ & $0.130 \%$ & $0.131 \%$ & $0.131 \%$ & $0.134 \%$ & $0.141 \%$ & $0.098 \%$ & $0.117 \%$ & $0.125 \%$ & $0.140 \%$ & $0.127 \%$ & $0.133 \%$ & $0.129 \%$ \\
\hline Zn & $0.015 \%$ & $0.008 \%$ & $0.009 \%$ & $0.009 \%$ & $0.009 \%$ & $0.010 \%$ & $0.009 \%$ & $0.009 \%$ & $0.010 \%$ & $0.010 \%$ & $0.011 \%$ & $0.011 \%$ & $0.011 \%$ \\
\hline Si & $0.478 \%$ & $0.164 \%$ & $0.432 \%$ & $0.282 \%$ & $0.198 \%$ & $0.191 \%$ & $0.118 \%$ & $0.393 \%$ & $0.182 \%$ & $0.338 \%$ & $0.197 \%$ & $0.316 \%$ & $0.347 \%$ \\
\hline Mn & $0.038 \%$ & $0.014 \%$ & $0.053 \%$ & $0.042 \%$ & $0.098 \%$ & $0.124 \%$ & $0.020 \%$ & $0.083 \%$ & $0.111 \%$ & $0.221 \%$ & $0.127 \%$ & $0.132 \%$ & $0.103 \%$ \\
\hline $\mathbf{F e}$ & $98.62 \%$ & $98.57 \%$ & $96.44 \%$ & $98.33 \%$ & $96.71 \%$ & $96.74 \%$ & $99.10 \%$ & $96.90 \%$ & $96.65 \%$ & $95.91 \%$ & $96.09 \%$ & $96.14 \%$ & $96.31 \%$ \\
\hline Mg & $0.001 \%$ & $0.003 \%$ & $0.002 \%$ & $0.002 \%$ & $0.002 \%$ & $0.002 \%$ & $0.002 \%$ & $0.001 \%$ & $0.002 \%$ & $0.004 \%$ & $0.002 \%$ & $0.002 \%$ & $0.002 \%$ \\
\hline $\mathbf{V}$ & $0.011 \%$ & $0.013 \%$ & $0.015 \%$ & $0.017 \%$ & $0.018 \%$ & $0.018 \%$ & $0.017 \%$ & $0.022 \%$ & $0.016 \%$ & $0.024 \%$ & $0.020 \%$ & $0.016 \%$ & $0.007 \%$ \\
\hline Al & $0.031 \%$ & $0.047 \%$ & $0.040 \%$ & $0.043 \%$ & $0.033 \%$ & $0.044 \%$ & $0.032 \%$ & $0.033 \%$ & $0.033 \%$ & $0.047 \%$ & $0.055 \%$ & $0.056 \%$ & $0.051 \%$ \\
\hline
\end{tabular}


Steel sample chemical analysis results

\begin{tabular}{|c|c|c|c|c|c|c|c|c|c|c|c|c|}
\hline $\begin{array}{c}\text { Sample } \\
\#\end{array}$ & Hd1-3 & Hd2-3 & Hd3-3 & He1-3 & He2-3 & He3-3 & Hf1-3 & Hf2-3 & Hf3-3 & Hg1-3 & Hg2-3 & Hg3-3 \\
\hline $\mathrm{Cr}$ & $0.074 \%$ & $0.070 \%$ & $0.064 \%$ & $0.077 \%$ & $0.074 \%$ & $0.077 \%$ & $0.071 \%$ & $0.063 \%$ & $0.072 \%$ & $0.075 \%$ & $0.089 \%$ & $0.082 \%$ \\
\hline $\mathbf{P}$ & $0.134 \%$ & $0.125 \%$ & $0.143 \%$ & $0.128 \%$ & $0.132 \%$ & $0.156 \%$ & $0.116 \%$ & $0.140 \%$ & $0.127 \%$ & $0.144 \%$ & $0.178 \%$ & $0.193 \%$ \\
\hline $\mathbf{Z n}$ & $0.012 \%$ & $0.011 \%$ & $0.013 \%$ & $0.011 \%$ & $0.012 \%$ & $0.011 \%$ & $0.011 \%$ & $0.012 \%$ & $0.012 \%$ & $0.011 \%$ & $0.011 \%$ & $0.012 \%$ \\
\hline Si & $0.339 \%$ & $0.349 \%$ & $0.275 \%$ & $0.178 \%$ & $0.374 \%$ & $0.200 \%$ & $0.252 \%$ & $0.382 \%$ & $0.229 \%$ & $0.106 \%$ & $0.174 \%$ & $0.477 \%$ \\
\hline Mn & $0.038 \%$ & $0.072 \%$ & $0.280 \%$ & $0.028 \%$ & $0.067 \%$ & $0.089 \%$ & $0.123 \%$ & $0.080 \%$ & $0.098 \%$ & $0.097 \%$ & $0.168 \%$ & $0.136 \%$ \\
\hline $\mathbf{F e}$ & $98.412 \%$ & $96.335 \%$ & $96.144 \%$ & $96.754 \%$ & $96.124 \%$ & $96.942 \%$ & $96.312 \%$ & $96.903 \%$ & $97.001 \%$ & $96.901 \%$ & $96.000 \%$ & $95.002 \%$ \\
\hline Mg & $0.002 \%$ & $0.002 \%$ & $0.010 \%$ & $0.002 \%$ & $0.002 \%$ & $0.002 \%$ & $0.005 \%$ & $0.002 \%$ & $0.002 \%$ & $0.002 \%$ & $0.003 \%$ & $0.002 \%$ \\
\hline $\mathbf{V}$ & $0.018 \%$ & $0.019 \%$ & $0.021 \%$ & $0.016 \%$ & $0.013 \%$ & $0.016 \%$ & $0.009 \%$ & $0.013 \%$ & $0.000 \%$ & $0.002 \%$ & $0.001 \%$ & $0.013 \%$ \\
\hline Al & $0.058 \%$ & $0.052 \%$ & $0.064 \%$ & $0.055 \%$ & $0.073 \%$ & $0.069 \%$ & $0.063 \%$ & $0.057 \%$ & $0.058 \%$ & $0.050 \%$ & $0.060 \%$ & $0.056 \%$ \\
\hline $\begin{array}{c}\text { Sample } \\
\#\end{array}$ & Hh1-3 & Hh2-3 & Hh3-3 & Hh4-3 & Hi1-3 & Hi2-3 & Hi3-3 & Hi4-3 & & & & \\
\hline $\mathrm{Cr}$ & $0.062 \%$ & $0.071 \%$ & $0.084 \%$ & $0.080 \%$ & $0.070 \%$ & $0.08 \%$ & $0.09 \%$ & $0.081 \%$ & & & & \\
\hline $\mathbf{P}$ & $0.138 \%$ & $0.144 \%$ & $0.164 \%$ & $0.146 \%$ & $0.119 \%$ & $0.15 \%$ & $0.15 \%$ & $0.143 \%$ & & & & \\
\hline Zn & $0.011 \%$ & $0.012 \%$ & $0.012 \%$ & $0.012 \%$ & $0.012 \%$ & $0.01 \%$ & $0.01 \%$ & $0.011 \%$ & & & & \\
\hline Si & $0.080 \%$ & $0.301 \%$ & $0.228 \%$ & $0.158 \%$ & $0.122 \%$ & $0.20 \%$ & $0.20 \%$ & $0.311 \%$ & & & & \\
\hline Mn & $0.009 \%$ & $0.095 \%$ & $0.110 \%$ & $0.149 \%$ & $0.019 \%$ & $0.11 \%$ & $0.07 \%$ & $0.097 \%$ & & & & \\
\hline $\mathbf{F e}$ & $98.413 \%$ & $97.001 \%$ & $96.903 \%$ & $97.242 \%$ & $99.500 \%$ & $98.56 \%$ & $98.30 \%$ & $96.904 \%$ & & & & \\
\hline Mg & $0.003 \%$ & $0.003 \%$ & $0.004 \%$ & $0.002 \%$ & $0.002 \%$ & $0.00 \%$ & $0.00 \%$ & $0.003 \%$ & & & & \\
\hline $\mathbf{V}$ & $0.001 \%$ & $0.015 \%$ & $0.000 \%$ & $0.002 \%$ & $0.012 \%$ & $0.03 \%$ & $0.03 \%$ & $0.009 \%$ & & & & \\
\hline $\mathbf{A l}$ & $0.063 \%$ & $0.064 \%$ & $0.072 \%$ & $0.053 \%$ & $0.048 \%$ & $0.06 \%$ & $0.07 \%$ & $0.065 \%$ & & & & \\
\hline
\end{tabular}


Slag sample chemical analysis results

\begin{tabular}{|c|c|c|c|c|c|c|c|c|c|c|c|c|}
\hline Oxides & Ma1 & Ma2 & Ma3 & Mb1 & Mb2 & Mb3 & Mb4 & Mb5 & Mc1 & Mc2 & Мc3 & Md1 \\
\hline $\mathrm{P} 2 \mathrm{O} 5$ & $0.340 \%$ & $0.390 \%$ & $0.184 \%$ & $0.480 \%$ & $0.465 \%$ & $0.357 \%$ & $0.129 \%$ & $0.156 \%$ & $0.279 \%$ & $0.124 \%$ & $0.164 \%$ & $0.207 \%$ \\
\hline $\mathrm{SiO} 2$ & $53.085 \%$ & $61.853 \%$ & $65.260 \%$ & $53.528 \%$ & $66.101 \%$ & $67.291 \%$ & $61.864 \%$ & $62.961 \%$ & $51.656 \%$ & $63.031 \%$ & $66.958 \%$ & $49.075 \%$ \\
\hline $\mathrm{MnO}$ & $0.195 \%$ & $0.300 \%$ & $0.205 \%$ & $0.181 \%$ & $0.353 \%$ & $0.224 \%$ & $0.293 \%$ & $0.329 \%$ & $0.201 \%$ & $0.318 \%$ & $0.234 \%$ & $0.154 \%$ \\
\hline $\mathrm{FeO}$ & $27.840 \%$ & $6.385 \%$ & $1.410 \%$ & $28.954 \%$ & $4.131 \%$ & $1.239 \%$ & $7.294 \%$ & $1.568 \%$ & $29.832 \%$ & $10.055 \%$ & $2.129 \%$ & $35.203 \%$ \\
\hline $\mathrm{MgO}$ & $2.103 \%$ & $3.618 \%$ & $3.739 \%$ & $1.856 \%$ & $4.224 \%$ & $3.313 \%$ & $3.614 \%$ & $4.428 \%$ & $2.015 \%$ & $3.224 \%$ & $3.672 \%$ & $1.645 \%$ \\
\hline $\mathrm{Al} 2 \mathrm{O} 3$ & $7.443 \%$ & $9.346 \%$ & $12.249 \%$ & $7.365 \%$ & $10.547 \%$ & $11.248 \%$ & $9.371 \%$ & $10.593 \%$ & $7.644 \%$ & $9.433 \%$ & $10.346 \%$ & $7.057 \%$ \\
\hline $\mathrm{TiO} 2$ & $0.173 \%$ & $0.245 \%$ & $0.306 \%$ & $0.172 \%$ & $0.248 \%$ & $0.249 \%$ & $0.245 \%$ & $0.271 \%$ & $0.155 \%$ & $0.216 \%$ & $0.251 \%$ & $0.144 \%$ \\
\hline $\mathrm{CaO}$ & $8.678 \%$ & $17.669 \%$ & $16.156 \%$ & $7.255 \%$ & $13.739 \%$ & $15.813 \%$ & $16.960 \%$ & $19.084 \%$ & $8.080 \%$ & $13.442 \%$ & $15.911 \%$ & $6.356 \%$ \\
\hline $\mathrm{C}$ & $0.075 \%$ & $0.120 \%$ & $0.252 \%$ & $0.143 \%$ & $0.114 \%$ & $0.125 \%$ & $0.127 \%$ & $0.453 \%$ & $0.111 \%$ & $0.105 \%$ & $0.187 \%$ & $0.087 \%$ \\
\hline $\mathrm{S}$ & $0.068 \%$ & $0.073 \%$ & $0.238 \%$ & $0.067 \%$ & $0.077 \%$ & $0.141 \%$ & $0.102 \%$ & $0.157 \%$ & $0.028 \%$ & $0.052 \%$ & $0.146 \%$ & $0.074 \%$ \\
\hline
\end{tabular}

Slag sample chemical analysis results

\begin{tabular}{|c|c|c|c|c|c|c|c|c|c|c|c|c|}
\hline Oxides & Md2 & Md3 & Me2 & Me3 & Mf1 & Mf2 & Mf3 & Mg1 & Mg2 & Mg3 & Mh1 & Mh2 \\
\hline $\mathrm{P} 2 \mathrm{O} 5$ & $0.248 \%$ & $0.287 \%$ & $0.193 \%$ & $0.171 \%$ & $0.165 \%$ & $0.283 \%$ & $0.403 \%$ & $0.343 \%$ & $0.258 \%$ & $0.276 \%$ & $0.334 \%$ & $0.347 \%$ \\
\hline $\mathrm{SiO} 2$ & $61.030 \%$ & $59.578 \%$ & $61.324 \%$ & $67.215 \%$ & $61.600 \%$ & $63.572 \%$ & $63.795 \%$ & $61.196 \%$ & $59.129 \%$ & $62.430 \%$ & $45.716 \%$ & $49.868 \%$ \\
\hline $\mathrm{MnO}$ & $0.272 \%$ & $0.235 \%$ & $0.364 \%$ & $0.272 \%$ & $0.339 \%$ & $0.333 \%$ & $0.342 \%$ & $0.282 \%$ & $0.268 \%$ & $0.207 \%$ & $0.140 \%$ & $0.201 \%$ \\
\hline $\mathrm{FeO}$ & $13.727 \%$ & $13.545 \%$ & $2.170 \%$ & $2.084 \%$ & $5.690 \%$ & $2.613 \%$ & $3.042 \%$ & $6.583 \%$ & $3.608 \%$ & $0.982 \%$ & $38.618 \%$ & $30.153 \%$ \\
\hline $\mathrm{MgO}$ & $2.388 \%$ & $3.422 \%$ & $4.691 \%$ & $3.876 \%$ & $4.332 \%$ & $4.383 \%$ & $4.476 \%$ & $3.764 \%$ & $3.314 \%$ & $2.679 \%$ & $1.587 \%$ & $2.207 \%$ \\
\hline $\mathrm{Al} 2 \mathrm{O} 3$ & $8.662 \%$ & $8.620 \%$ & $8.739 \%$ & $11.295 \%$ & $9.139 \%$ & $9.247 \%$ & $9.276 \%$ & $8.808 \%$ & $8.933 \%$ & $9.411 \%$ & $6.301 \%$ & $7.647 \%$ \\
\hline $\mathrm{TiO} 2$ & $0.215 \%$ & $0.238 \%$ & $0.277 \%$ & $0.255 \%$ & $0.259 \%$ & $0.277 \%$ & $0.241 \%$ & $0.226 \%$ & $0.196 \%$ & $0.197 \%$ & $0.134 \%$ & $0.156 \%$ \\
\hline $\mathrm{CaO}$ & $13.262 \%$ & $13.828 \%$ & $21.805 \%$ & $14.663 \%$ & $18.261 \%$ & $19.062 \%$ & $18.149 \%$ & $18.506 \%$ & $23.969 \%$ & $23.475 \%$ & $6.756 \%$ & $9.057 \%$ \\
\hline $\mathrm{C}$ & $0.132 \%$ & $0.131 \%$ & $0.280 \%$ & $0.107 \%$ & $0.123 \%$ & $0.124 \%$ & $0.174 \%$ & $0.138 \%$ & $0.130 \%$ & $0.136 \%$ & $0.074 \%$ & $0.096 \%$ \\
\hline $\mathrm{S}$ & $0.064 \%$ & $0.116 \%$ & $0.157 \%$ & $0.064 \%$ & $0.091 \%$ & $0.106 \%$ & $0.103 \%$ & $0.153 \%$ & $0.194 \%$ & $0.206 \%$ & $0.340 \%$ & $0.268 \%$ \\
\hline
\end{tabular}


Slag sample chemical analysis results

\begin{tabular}{|c|c|c|c|c|c|c|c|c|c|c|c|c|}
\hline Oxides & Mh3 & Mh4 & Mh5 & Mi0 & Mi1 & Mj1 & Mj2 & Mj3 & Mk1 & Mk3 & Ml1 & Ml3 \\
\hline $\mathrm{P} 2 \mathrm{O} 5$ & $0.288 \%$ & $0.179 \%$ & $0.171 \%$ & $0.013 \%$ & $0.075 \%$ & $0.056 \%$ & $0.199 \%$ & $0.016 \%$ & $0.081 \%$ & $0.261 \%$ & $0.261 \%$ & $0.071 \%$ \\
\hline $\mathrm{SiO} 2$ & $60.116 \%$ & $61.023 \%$ & $48.388 \%$ & $61.132 \%$ & $61.064 \%$ & $53.227 \%$ & $57.537 \%$ & $63.293 \%$ & $51.550 \%$ & $53.350 \%$ & $52.302 \%$ & $65.792 \%$ \\
\hline $\mathrm{MnO}$ & $0.375 \%$ & $0.191 \%$ & $0.149 \%$ & $0.383 \%$ & $0.312 \%$ & $0.221 \%$ & $0.374 \%$ & $0.175 \%$ & $0.184 \%$ & $0.262 \%$ & $0.214 \%$ & $0.169 \%$ \\
\hline $\mathrm{FeO}$ & $5.660 \%$ & $2.436 \%$ & $9.329 \%$ & $1.781 \%$ & $1.171 \%$ & $25.125 \%$ & $8.310 \%$ & $3.276 \%$ & $30.980 \%$ & $11.288 \%$ & $28.476 \%$ & $2.593 \%$ \\
\hline $\mathrm{MgO}$ & $4.208 \%$ & $3.319 \%$ & $2.807 \%$ & $5.122 \%$ & $4.694 \%$ & $2.399 \%$ & $4.148 \%$ & $3.604 \%$ & $1.923 \%$ & $3.793 \%$ & $2.172 \%$ & $3.268 \%$ \\
\hline $\mathrm{Al} 2 \mathrm{O} 3$ & $8.795 \%$ & $12.160 \%$ & $9.195 \%$ & $8.252 \%$ & $9.485 \%$ & $8.098 \%$ & $8.664 \%$ & $12.329 \%$ & $7.254 \%$ & $8.716 \%$ & $7.322 \%$ & $11.589 \%$ \\
\hline $\mathrm{TiO} 2$ & $0.225 \%$ & $0.287 \%$ & $0.246 \%$ & $0.213 \%$ & $0.196 \%$ & $0.178 \%$ & $0.248 \%$ & $0.254 \%$ & $0.134 \%$ & $0.245 \%$ & $0.146 \%$ & $0.281 \%$ \\
\hline $\mathrm{CaO}$ & $19.999 \%$ & $17.392 \%$ & $14.510 \%$ & $22.946 \%$ & $22.824 \%$ & $10.544 \%$ & $20.234 \%$ & $15.901 \%$ & $7.750 \%$ & $18.248 \%$ & $8.913 \%$ & $15.664 \%$ \\
\hline $\mathrm{C}$ & $0.119 \%$ & $2.630 \%$ & $14.500 \%$ & $0.127 \%$ & $0.147 \%$ & $0.085 \%$ & \begin{tabular}{|l}
$0.117 \%$ \\
\end{tabular} & $0.807 \%$ & $0.079 \%$ & $3.490 \%$ & $0.085 \%$ & $0.301 \%$ \\
\hline $\mathrm{S}$ & $0.216 \%$ & $0.383 \%$ & $0.705 \%$ & $0.029 \%$ & $0.032 \%$ & $0.067 \%$ & $0.169 \%$ & $0.345 \%$ & $0.064 \%$ & $0.348 \%$ & $0.110 \%$ & $0.272 \%$ \\
\hline
\end{tabular}


APPENDIX B: Summary of MW/EAF steelmaking results

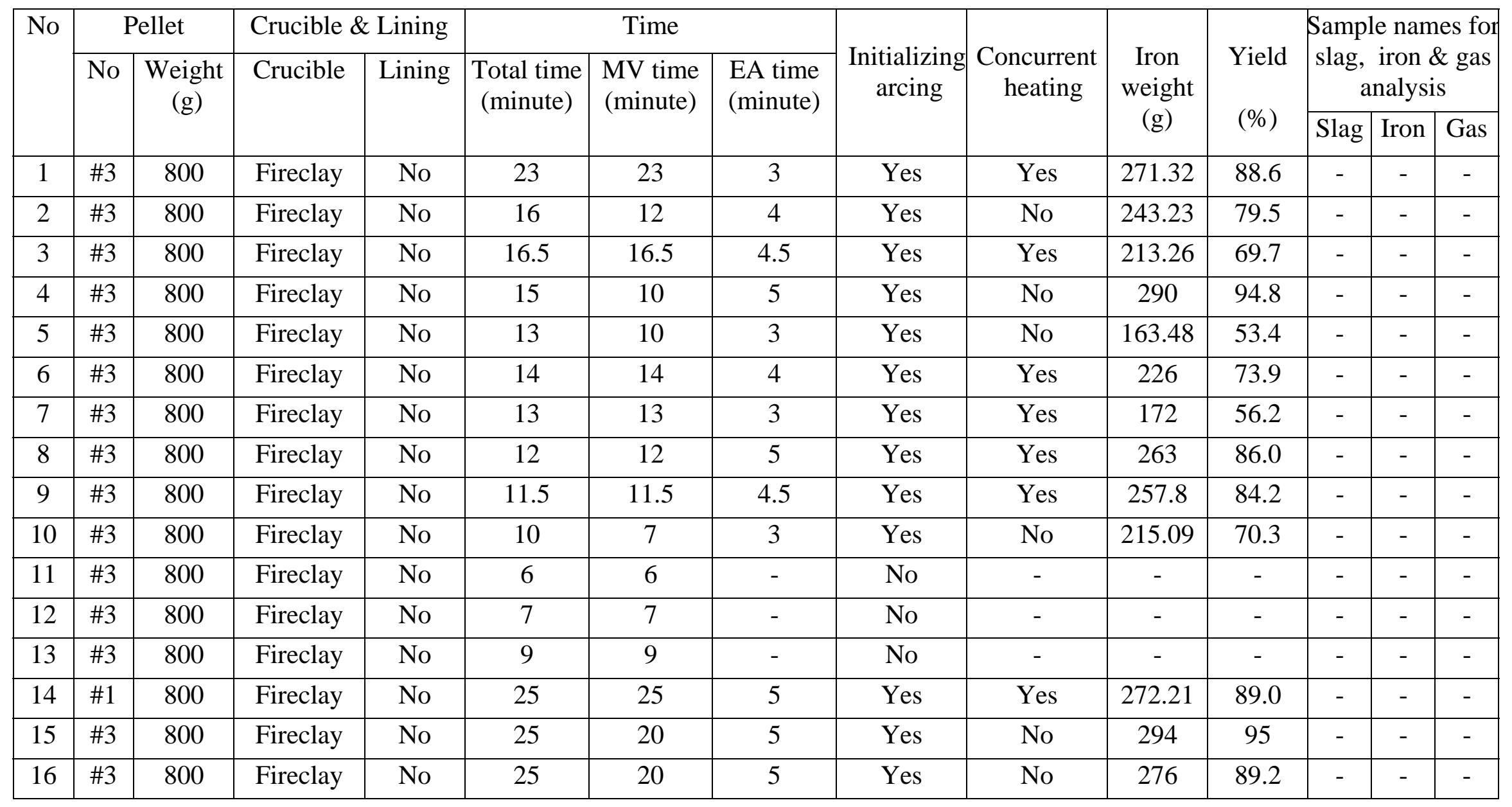




\section{Appendix B Continued}

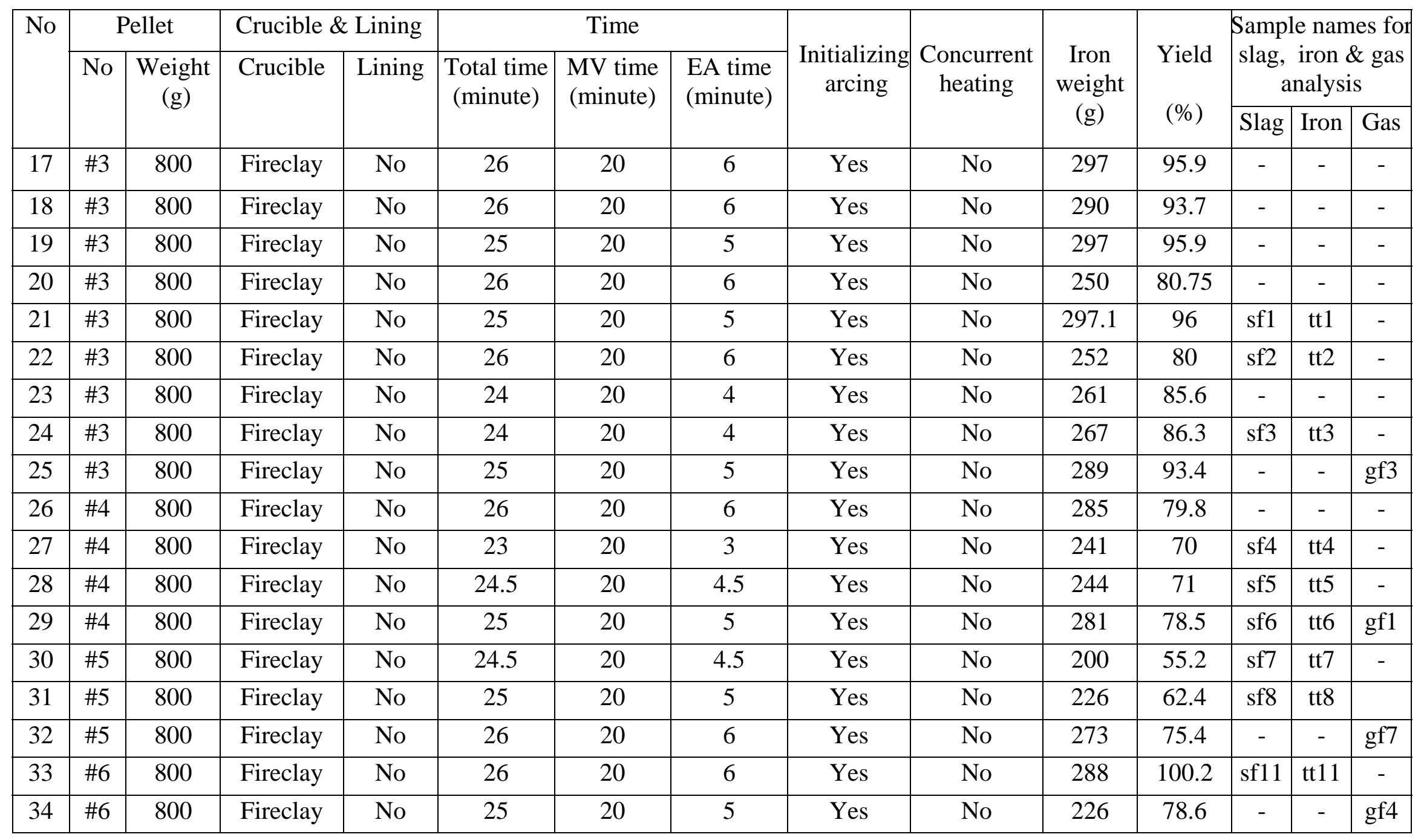




\section{Appendix B Continued}

\begin{tabular}{|c|c|c|c|c|c|c|c|c|c|c|c|c|c|c|}
\hline \multirow[t]{3}{*}{ No } & \multicolumn{2}{|c|}{ Pellet } & \multicolumn{2}{|c|}{ Crucible \& Lining } & \multicolumn{3}{|c|}{ Time } & \multirow{3}{*}{$\begin{array}{c}\text { Initializing } \\
\text { arcing }\end{array}$} & \multirow{3}{*}{$\begin{array}{c}\text { Concurrent } \\
\text { heating }\end{array}$} & \multirow{3}{*}{$\begin{array}{l}\text { Iron } \\
\text { weight } \\
\text { (g) }\end{array}$} & \multirow{3}{*}{$\begin{array}{l}\text { Yield } \\
\text { (\%) }\end{array}$} & \multirow{2}{*}{\multicolumn{3}{|c|}{$\begin{array}{l}\text { Sample names for } \\
\text { slag, iron \& gas } \\
\text { analysis }\end{array}$}} \\
\hline & \multirow[t]{2}{*}{ No } & \multirow{2}{*}{$\begin{array}{c}\text { Weight } \\
\text { (g) }\end{array}$} & \multirow[t]{2}{*}{ Crucible } & \multirow[t]{2}{*}{ Lining } & \multirow{2}{*}{$\begin{array}{l}\text { Total time } \\
\text { (minute) }\end{array}$} & \multirow{2}{*}{$\begin{array}{l}\text { MV time } \\
\text { (minute) }\end{array}$} & \multirow{2}{*}{$\begin{array}{l}\text { EA time } \\
\text { (minute) }\end{array}$} & & & & & & & \\
\hline & & & & & & & & & & & & Slag & Iron & Gas \\
\hline 35 & $\# 7$ & 800 & Fireclay & No & 27 & 20 & 7 & Yes & No & 261 & 95.1 & sf9 & $\mathrm{tt} 9$ & - \\
\hline 36 & \#7 & 800 & Fireclay & No & 20 & 20 & - & No & - & - & - & - & - & gf5 \\
\hline 39 & $\# 2$ & 800 & Fireclay & No & 25 & 20 & 5 & Yes & No & 268 & 81.4 & - & - & gf6 \\
\hline 40 & $\# 1$ & 800 & Fireclay & No & 25 & 20 & 5 & Yes & No & 260 & 68.8 & - & - & gf8 \\
\hline 41 & $\# 9$ & 800 & Fireclay & No & 26 & 20 & 6 & Yes & No & 302 & 96.2 & - & - & gf9 \\
\hline
\end{tabular}

\begin{tabular}{|c|c|c|c|c|c|c|c|c|c|c|c|c|c|c|}
\hline 42 & $\# 3$ & 600 & $\mathrm{MgO}$ & No & 26 & 20 & 6 & Yes & No & 167.7 & 72.3 & sd1 & td1 & - \\
\hline 43 & $\# 3$ & 600 & $\mathrm{MgO}$ & No & 26 & 20 & 6 & Yes & No & 152 & 65.5 & - & - & - \\
\hline 44 & \#3 & 600 & $\mathrm{MgO}$ & Cao & 26 & 20 & 6 & Yes & No & 211 & 91.0 & sd3 & td3 & - \\
\hline 46 & $\# 9$ & 600 & $\mathrm{MgO}$ & No & 26 & 20 & 6 & Yes & No & 177 & 75.2 & sd5 & td5 & - \\
\hline 47 & $\# 9$ & 600 & $\mathrm{MgO}$ & Cao & 26 & 20 & 6 & Yes & No & 220 & 93.4 & sd6 & td6 & - \\
\hline 50 & $\# 9$ & 600 & Fireclay & $\mathrm{MgO}$ & 26 & 20 & 6 & Yes & No & 227 & 96.4 & sd9 & td9 & - \\
\hline 51 & $\# 10$ & 600 & Fireclay & $\mathrm{MgO}$ & 26 & 20 & 6 & Yes & No & 232 & 101.5 & sd10 & td10 & - \\
\hline
\end{tabular}




\section{APPENDIX C: Composition of Offgases in the MW/EAF Process}

\begin{tabular}{|c|c|c|c|c|c|}
\hline \multicolumn{6}{|c|}{ Gas sample: gf1 } \\
\hline & $\mathrm{N}_{2} \%$ & $\mathrm{CO}_{2} \quad \%$ & $\mathrm{O}_{2} \quad \%$ & $\mathrm{SO}_{2} \quad \%$ & $\mathrm{H}_{2} \mathrm{O} \%$ \\
\hline & \multicolumn{5}{|c|}{ Microwave Heating (total time 20 min) } \\
\hline $2 \min$ & 76.1 & 7.07 & 16.4 & 0.0390 & 0.312 \\
\hline $4 \min$ & 76.7 & 7.07 & 15.9 & 0.0209 & 0.309 \\
\hline $6 \mathrm{~min}$ & 76.6 & 6.75 & 16.3 & 0.0212 & 0.326 \\
\hline $8 \mathrm{~min}$ & 76.6 & 6.68 & 16.0 & 0.0208 & 0.719 \\
\hline $10 \min$ & 77.2 & 7.06 & 15.3 & 0.0121 & 0.483 \\
\hline $12 \min$ & 79.6 & 7.44 & 12.4 & 0.0207 & 0.498 \\
\hline $15 \min$ & 78.3 & 8.24 & 12.8 & 0.0256 & 0.681 \\
\hline $18 \mathrm{~min}$ & 78.1 & 8.30 & 12.7 & 0.0289 & 0.849 \\
\hline \multirow[t]{2}{*}{$20 \mathrm{~min}$} & 78.1 & 7.96 & 12.7 & 0.0255 & 1.19 \\
\hline & \multicolumn{5}{|c|}{ Electric Arc Heating (total time 5 min) } \\
\hline $2 \min$ & 78.3 & 10.3 & 9.46 & 0.0138 & 1.89 \\
\hline $4 \min$ & 78.4 & 9.46 & 10.3 & 0.00 & 1.80 \\
\hline
\end{tabular}

\begin{tabular}{|c|c|c|c|c|c|}
\hline \multicolumn{6}{|c|}{ Gas sample: gf2 } \\
\hline & $\mathrm{N}_{2} \%$ & $\mathrm{CO}_{2} \%$ & $\mathrm{O}_{2} \%$ & $\mathrm{SO}_{2} \%$ & $\mathrm{H}_{2} \mathrm{O} \%$ \\
\hline & \multicolumn{5}{|c|}{ Microwave Heating (total time 20 min) } \\
\hline $1 \mathrm{~min}$ & 75.5 & 3.80 & 20.0 & 0.0661 & 0.614 \\
\hline $3 \mathrm{~min}$ & 76.9 & 3.05 & 19.2 & 0.0231 & 0.829 \\
\hline $5 \min$ & 78.9 & 10.7 & 9.32 & 0.0215 & 1.06 \\
\hline $7 \mathrm{~min}$ & 78.2 & 9.93 & 10.7 & 0.0434 & 1.19 \\
\hline $9 \min$ & 76.8 & 12.5 & 9.07 & 0.0426 & 1.59 \\
\hline $12 \min$ & 76.1 & 13.1 & 8.99 & 0.0104 & 1.78 \\
\hline $15 \mathrm{~min}$ & 75.0 & 12.9 & 10.2 & 0.00 & 1.89 \\
\hline $17 \mathrm{~min}$ & 76.5 & 11.5 & 10.2 & 0.0191 & 1.83 \\
\hline \multirow[t]{2}{*}{$20 \mathrm{~min}$} & 76.4 & 10.8 & 10.8 & 0.0191 & 1.96 \\
\hline & \multicolumn{5}{|c|}{ Electric Arc Heating (total time 5 min) } \\
\hline $2 \min$ & 78.2 & 10.7 & 9.87 & 0.00 & 1.23 \\
\hline $4 \mathrm{~min}$ & 77.8 & 10.6 & 10.2 & 0.0320 & 1.31 \\
\hline
\end{tabular}




\section{Appendix C continued}

\begin{tabular}{|c|c|c|c|c|c|}
\hline \multicolumn{6}{|c|}{ Gas sample: gf3 } \\
\hline & $\mathrm{N}_{2} \%$ & $\mathrm{CO}_{2} \%$ & $\mathrm{O}_{2} \quad \%$ & $\mathrm{SO}_{2} \quad \%$ & $\mathrm{H}_{2} \mathrm{O} \%$ \\
\hline & \multicolumn{5}{|c|}{ Microwave Heating (total time 20 min) } \\
\hline $1 \mathrm{~min}$ & 83.9 & 5.89 & 7.34 & 2.90 & 0.000 \\
\hline $3 \mathrm{~min}$ & 81.5 & 9.49 & 8.14 & 0.1120 & 0.815 \\
\hline $5 \mathrm{~min}$ & 79.8 & 9.41 & 9.42 & 0.0689 & 1.31 \\
\hline $7 \mathrm{~min}$ & 79.8 & 8.68 & 10.1 & 0.0334 & 1.38 \\
\hline $9 \mathrm{~min}$ & 78.4 & 7.35 & 12.5 & 0.0243 & 1.65 \\
\hline $11 \mathrm{~min}$ & 80.5 & 6.10 & 11.9 & 0.0421 & 1.46 \\
\hline $13 \mathrm{~min}$ & 78.2 & 6.44 & 13.5 & 0.0323 & 1.77 \\
\hline $15 \mathrm{~min}$ & 78.7 & 5.85 & 13.6 & 0.0483 & 1.78 \\
\hline $17 \min$ & 78.7 & 5.47 & 14.0 & 0.0496 & 1.83 \\
\hline \multirow[t]{2}{*}{$19 \mathrm{~min}$} & 79.3 & 4.68 & 13.9 & 0.0815 & 2.04 \\
\hline & \multicolumn{5}{|c|}{ Electric Arc Heating (total time 5 min) } \\
\hline $2 \min$ & 76.5 & 15.5 & 7.19 & 0.0166 & 0.832 \\
\hline $4 \mathrm{~min}$ & 75.3 & 14.2 & 9.25 & 0.0000 & 1.19 \\
\hline
\end{tabular}

\begin{tabular}{|c|c|c|c|c|c|}
\hline \multicolumn{6}{|c|}{ Gas sample: gf4 } \\
\hline & $\mathrm{N}_{2} \quad \%$ & $\mathrm{CO}_{2} \quad \%$ & $\mathrm{O}_{2} \%$ & $\mathrm{SO}_{2} \quad \%$ & $\mathrm{H}_{2} \mathrm{O} \%$ \\
\hline & \multicolumn{5}{|c|}{ Microwave Heating (total time $20 \mathrm{~min}$ ) } \\
\hline $2 \mathrm{~min}$ & 75.5 & 12.6 & 11.1 & 0.0189 & 0.910 \\
\hline $5 \mathrm{~min}$ & 77.9 & 10.2 & 10.3 & 0.308 & 1.32 \\
\hline $7 \mathrm{~min}$ & 76.2 & 10.5 & 11.8 & 0.00 & 1.53 \\
\hline $9 \mathrm{~min}$ & 76.0 & 9.68 & 12.7 & 0.00 & 1.71 \\
\hline $11 \mathrm{~min}$ & 74.7 & 9.87 & 13.7 & 0.00 & 1.71 \\
\hline $13 \mathrm{~min}$ & 74.8 & 9.75 & 13.5 & 0.00 & 1.90 \\
\hline $15 \mathrm{~min}$ & 74.9 & 9.55 & 13.5 & 0.00 & 2.00 \\
\hline $17 \min$ & 76.0 & 9.36 & 12.7 & 0.00 & 2.03 \\
\hline \multirow[t]{2}{*}{$19 \mathrm{~min}$} & 77.9 & 8.59 & 11.4 & 0.00 & 2.10 \\
\hline & \multicolumn{5}{|c|}{ Electric Arc Heating (total time 5 min) } \\
\hline $2 \min$ & 77.1 & 10.1 & 10.2 & 0.004 & 2.63 \\
\hline $4 \min$ & 77.2 & 9.73 & 10.5 & 0.007 & 2.55 \\
\hline
\end{tabular}




\section{Appendix C continued}

Gas sample: gf5

\begin{tabular}{|c|c|c|c|c|c|c|c|c|}
\hline & $\mathrm{N}_{2} \%$ & $\mathrm{CO}_{2}$ & $\mathrm{O}_{2}$ & $\mathrm{SO}_{2}$ & $\%$ & $\mathrm{H}_{2} \mathrm{O} \%$ & $\mathrm{Ar} \%$ & $\mathrm{CO} \%$ \\
\cline { 2 - 8 } & \multicolumn{7}{|c|}{ Microwave Heating (total time 20 min) } \\
\hline $2 \mathrm{~min}$ & 78.12 & 4.50 & 15.09 & 0.0373 & 1.03 & 1.23 & 0.0321 \\
\hline $4 \mathrm{~min}$ & 76.85 & 6.24 & 14.47 & 0.0280 & 1.33 & 1.09 & 0.0133 \\
\hline $6 \mathrm{~min}$ & 77.99 & 7.46 & 11.85 & 0.0323 & 1.57 & 1.10 & 0 \\
\hline $8 \mathrm{~min}$ & 79.93 & 5.52 & 11.52 & 0.0170 & 1.87 & 1.14 & 0 \\
\hline $10 \mathrm{~min}$ & 80.02 & 4.97 & 11.85 & 0.0028 & 2.00 & 1.15 & 0 \\
\hline $12 \mathrm{~min}$ & 79.37 & 5.29 & 12.17 & 0.0027 & 2.03 & 1.14 & 0 \\
\hline $14 \mathrm{~min}$ & 79.75 & 4.42 & 12.44 & 0.0028 & 2.25 & 1.13 & 0 \\
\hline $16 \mathrm{~min}$ & 79.63 & 4.43 & 12.53 & 0.0027 & 2.29 & 1.13 & 0 \\
\hline $18 \mathrm{~min}$ & 79.58 & 4.60 & 12.34 & 0.0027 & 2.35 & 1.13 & 0 \\
\hline $20 \mathrm{~min}$ & 79.73 & 4.60 & 12.09 & 0.0030 & 2.44 & 1.13 & 0 \\
\hline
\end{tabular}

Gas sample: gf6

\begin{tabular}{|c|c|c|c|c|c|c|c|}
\hline & $\mathrm{N}_{2} \quad \%$ & $\mathrm{CO}_{2} \quad \%$ & $\mathrm{O}_{2} \quad \%$ & $\mathrm{SO}_{2} \quad \%$ & $\mathrm{H}_{2} \mathrm{O} \%$ & Ar \% & $\mathrm{CO} \%$ \\
\hline & \multicolumn{7}{|c|}{ Microwave Heating (total time $20 \mathrm{~min}$ ) } \\
\hline $2 \min$ & 78.67 & 1.85 & 17.34 & 0.0135 & 0.92 & 1.20 & 0.0423 \\
\hline $4 \min$ & 76.68 & 5.22 & 15.70 & 0.0264 & 1.22 & 1.15 & 0.0215 \\
\hline $6 \mathrm{~min}$ & 76.40 & 5.10 & 16.16 & 0.0265 & 1.17 & 1.15 & 0 \\
\hline $8 \mathrm{~min}$ & 75.95 & 5.85 & 15.53 & 0.0397 & 1.51 & 1.12 & 0 \\
\hline $10 \mathrm{~min}$ & 80.05 & 4.86 & 12.07 & 0.0419 & 1.81 & 1.17 & 0 \\
\hline $12 \mathrm{~min}$ & 78.90 & 6.78 & 11.35 & 0.0131 & 1.79 & 1.16 & 0 \\
\hline $14 \mathrm{~min}$ & 79.81 & 6.10 & 11.13 & 0.0001 & 1.80 & 1.16 & 0 \\
\hline $16 \mathrm{~min}$ & 79.22 & 4.98 & 12.68 & 0.0265 & 1.91 & 1.18 & 0 \\
\hline $18 \mathrm{~min}$ & 78.96 & 5.46 & 12.36 & 0.0133 & 1.98 & 1.22 & 0 \\
\hline \multirow[t]{2}{*}{$20 \mathrm{~min}$} & 79.73 & 5.65 & 11.39 & 0.0404 & 1.98 & 1.21 & 0 \\
\hline & \multicolumn{7}{|c|}{ Electric Arc Heating (total time 5 min) } \\
\hline $2 \min$ & 79.28 & 8.45 & 9.32 & 0.0021 & 1.80 & 1.14 & 0 \\
\hline $4 \mathrm{~min}$ & 78.56 & 9.14 & 9.26 & 0.0112 & 1.79 & 1.23 & 0 \\
\hline
\end{tabular}




\section{Appendix C continued}

Gas sample: gf7

\begin{tabular}{|c|c|c|c|c|c|c|c|c|}
\hline & $\mathrm{N}_{2} \%$ & $\mathrm{CO}_{2} \%$ & $\mathrm{O}_{2} \%$ & $\mathrm{SO}_{2} \%$ & $\mathrm{H}_{2} \mathrm{O} \%$ & $\mathrm{Ar} \%$ & $\mathrm{CO} \%$ \\
\cline { 2 - 8 } & \multicolumn{7}{|c|}{ Microwave Heating (total time 20 min) } \\
\hline $2 \mathrm{~min}$ & 77.01 & 0.59 & 19.14 & 0.0226 & 1.68 & 1.56 & 0.0105 \\
\hline $4 \mathrm{~min}$ & 76.25 & 3.82 & 16.48 & 0.0230 & 1.96 & 1.46 & 0.0132 \\
\hline $6 \mathrm{~min}$ & 75.20 & 6.66 & 13.94 & 0.0102 & 2.63 & 1.56 & 0 \\
\hline $8 \mathrm{~min}$ & 75.73 & 7.39 & 12.70 & 0.0353 & 2.64 & 1.51 & 0 \\
\hline $10 \mathrm{~min}$ & 75.45 & 7.26 & 13.20 & 0.0168 & 2.53 & 1.54 & 0 \\
\hline $12 \mathrm{~min}$ & 77.24 & 6.53 & 12.25 & 0.0117 & 2.46 & 1.50 & 0 \\
\hline $14 \mathrm{~min}$ & 76.37 & 6.10 & 13.63 & 0.0266 & 2.41 & 1.47 & 0 \\
\hline $16 \mathrm{~min}$ & 76.31 & 6.58 & 12.99 & 0.0246 & 2.57 & 1.53 & 0 \\
\hline $18 \mathrm{~min}$ & 76.93 & 6.29 & 12.58 & 0.0203 & 2.69 & 1.49 & 0 \\
\hline $20 \mathrm{~min}$ & 77.42 & 6.38 & 12.31 & 0.0169 & 2.37 & 1.50 & 0 \\
\hline & 75.86 & 10.23 & 10.46 & 0.0111 & 1.80 & 1.64 & 0 \\
\hline $2 \mathrm{~min}$ & 75.92 & 10.35 & 9.79 & 0.0104 & 2.21 & 1.71 & 0 \\
\hline $4 \mathrm{~min}$ & 78
\end{tabular}

\begin{tabular}{|c|c|c|c|c|c|c|c|}
\hline \multicolumn{8}{|c|}{ Gas sample: gf8 } \\
\hline & $\mathrm{N}_{2} \%$ & $\mathrm{CO}_{2} \quad \%$ & $\mathrm{O}_{2} \%$ & $\mathrm{SO}_{2} \%$ & $\mathrm{H}_{2} \mathrm{O} \%$ & $\operatorname{Ar} \%$ & $\mathrm{CO} \%$ \\
\hline & \multicolumn{7}{|c|}{ Microwave Heating (total time $20 \mathrm{~min}$ ) } \\
\hline $2 \mathrm{~min}$ & 72.82 & 5.15 & 19.40 & 0.0504 & 0.98 & 1.59 & 0.0276 \\
\hline $4 \mathrm{~min}$ & 76.61 & 4.22 & 16.68 & 0.0264 & 1.28 & 1.18 & 0.0149 \\
\hline $6 \mathrm{~min}$ & 76.04 & 5.71 & 15.46 & 0.0527 & 1.57 & 1.17 & 0.0050 \\
\hline $8 \mathrm{~min}$ & 75.89 & 5.70 & 15.34 & 0.0538 & 1.86 & 1.15 & 0 \\
\hline $10 \mathrm{~min}$ & 77.54 & 4.24 & 14.98 & 0.0271 & 2.07 & 1.13 & 0 \\
\hline $12 \mathrm{~min}$ & 74.55 & 6.33 & 15.97 & 0.0125 & 2.02 & 1.12 & 0 \\
\hline $14 \mathrm{~min}$ & 76.19 & 4.24 & 16.36 & 0.0126 & 2.06 & 1.14 & 0 \\
\hline $16 \mathrm{~min}$ & 73.67 & 7.68 & 15.51 & 0.0242 & 2.02 & 1.09 & 0 \\
\hline $18 \min$ & 73.73 & 7.20 & 15.89 & 0.0238 & 2.05 & 1.11 & 0 \\
\hline \multirow[t]{2}{*}{$20 \min$} & 75.91 & 5.86 & 15.01 & 0.0363 & 2.06 & 1.12 & 0 \\
\hline & \multicolumn{7}{|c|}{ Electric Arc Heating (total time $5 \mathrm{~min}$ ) } \\
\hline $2 \min$ & 75.22 & 9.10 & 13.56 & 0.0317 & 2.09 & 1.28 & 0 \\
\hline $4 \mathrm{~min}$ & 74.22 & 9.71 & 13.39 & 0.0275 & 2.65 & 1.28 & 0 \\
\hline
\end{tabular}




\section{Appendix C continued}

Gas sample: gf9

\begin{tabular}{|c|c|c|c|c|c|c|c|}
\hline & $\mathrm{N}_{2} \quad \%$ & $\mathrm{CO}_{2} \%$ & $\mathrm{O}_{2} \%$ & $\mathrm{SO}_{2} \quad \%$ & $\mathrm{H}_{2} \mathrm{O} \%$ & Ar \% & $\mathrm{CO} \%$ \\
\hline & \multicolumn{7}{|c|}{ Microwave Heating (total time $20 \mathrm{~min}$ ) } \\
\hline $2 \mathrm{~min}$ & 76.59 & 3.85 & 18.49 & 0.0317 & 1.04 & 1.51 & 0.0194 \\
\hline $4 \mathrm{~min}$ & 76.67 & 3.42 & 18.96 & 0.0172 & 0.93 & 1.45 & 0 \\
\hline $6 \mathrm{~min}$ & 80.38 & 6.68 & 11.61 & 0.0179 & 1.32 & 1.31 & 0 \\
\hline $8 \mathrm{~min}$ & 78.38 & 7.22 & 12.92 & 0.0353 & 1.45 & 1.22 & 0 \\
\hline $10 \mathrm{~min}$ & 78.63 & 7.19 & 12.28 & 0.0336 & 1.87 & 1.20 & 0 \\
\hline $12 \mathrm{~min}$ & 79.39 & 7.57 & 11.21 & 0.0259 & 1.79 & 1.27 & 0 \\
\hline $14 \mathrm{~min}$ & 78.71 & 6.74 & 12.83 & 0.0257 & 1.69 & 1.17 & 0 \\
\hline $16 \mathrm{~min}$ & 77.00 & 8.10 & 13.25 & 0.0249 & 1.64 & 1.17 & 0 \\
\hline $18 \mathrm{~min}$ & 76.17 & 8.01 & 14.07 & 0.0166 & 1.72 & 1.13 & 0 \\
\hline \multirow[t]{2}{*}{$20 \mathrm{~min}$} & 76.35 & 8.02 & 13.81 & 0.0122 & 1.81 & 1.19 & 0 \\
\hline & \multicolumn{7}{|c|}{ Electric Arc Heating (total time 6 min) } \\
\hline $2 \min$ & 79.38 & 10.11 & 8.23 & 0.0204 & 2.26 & 1.19 & 0 \\
\hline $4 \min$ & 78.36 & 11.00 & 7.92 & 0.0277 & 2.70 & 1.26 & 0 \\
\hline
\end{tabular}

Gas sample: from coal

\begin{tabular}{|c|c|c|c|c|c|}
\hline & $\mathrm{N}_{2} \%$ & $\mathrm{CO}_{2} \%$ & $\mathrm{O}_{2} \%$ & $\mathrm{SO}_{2} \%$ & $\mathrm{H}_{2} \mathrm{O} \%$ \\
\cline { 2 - 6 } & \multicolumn{5}{|c|}{ Microwave Heating (total time 20 min) } \\
\hline $1.5 \mathrm{~min}$ & 77.8 & 0.889 & 19.4 & 0.227 & 1.62 \\
\hline $3 \mathrm{~min}$ & 80.1 & 0.768 & 17.6 & 0.191 & 1.32 \\
\hline $5 \mathrm{~min}$ & 81.6 & 0.639 & 16.3 & 0.0730 & 1.39 \\
\hline $8 \min$ & 80.7 & 0.655 & 16.7 & 0.103 & 1.82 \\
\hline $11 \mathrm{~min}$ & 80.5 & 1.31 & 16.1 & 0.109 & 2.04 \\
\hline $14 \min$ & 79.7 & 2.24 & 15.9 & 0.155 & 2.02 \\
\hline $16 \min$ & 79.7 & 3.93 & 14.1 & 0.155 & 2.11 \\
\hline $19 \min$ & 77.6 & 5.77 & 14.4 & 0.149 & 2.03 \\
\hline
\end{tabular}


Appendix D: Table.D.1 . Microwave DRI Heat Balance, Preliminary Operating Cost

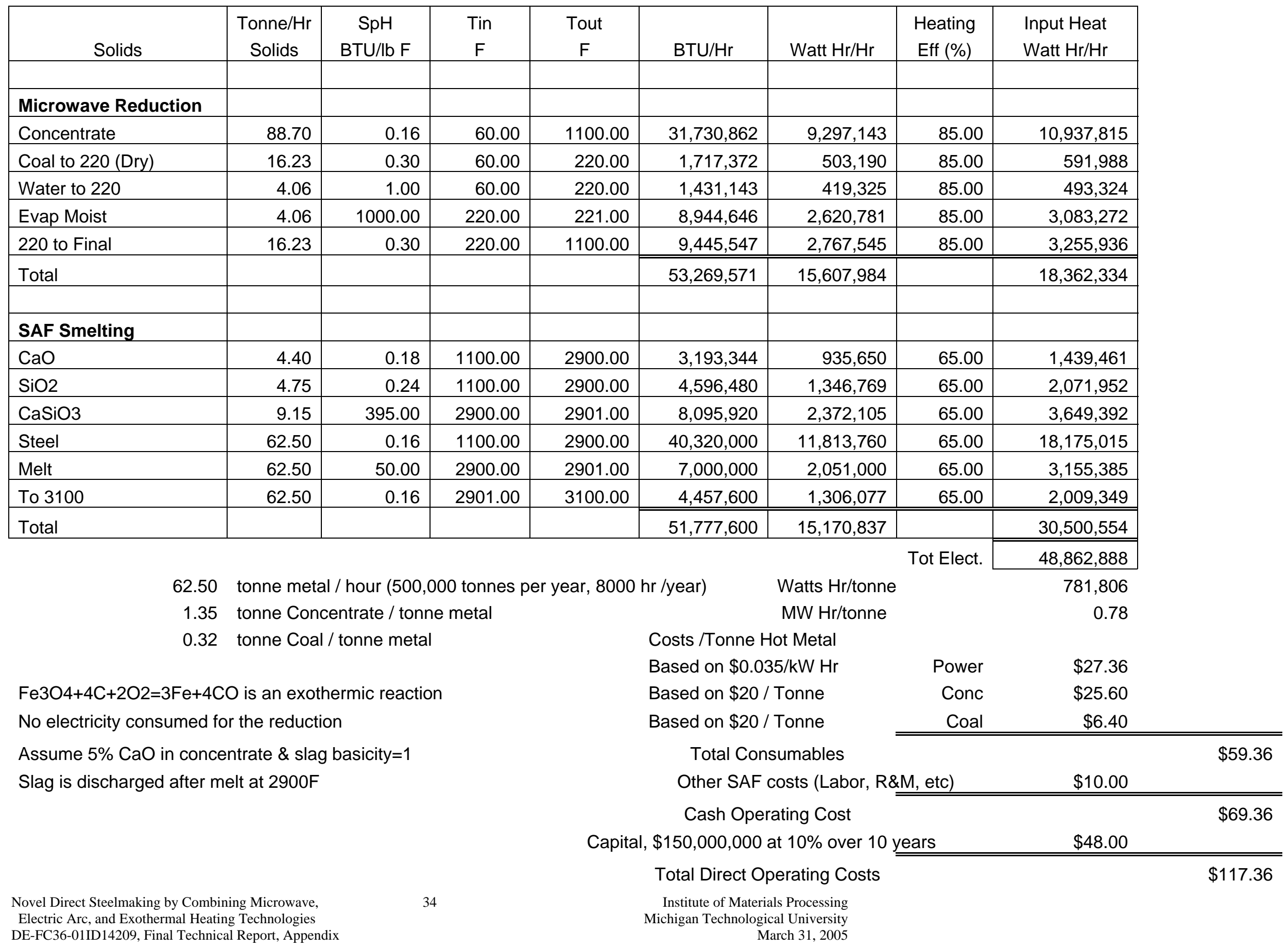




\section{Appendix D: Table D.2. Operating Costs}

\section{Conversion to Steel}

\section{Hot Metal (Cash Cost)}

\section{Scrap (Purchased and Reverts)}

Alloys

\section{Oxygen}

Flux

\section{Energy}

R\&M Supplies

Labor

\begin{tabular}{|c|c|}
\hline Labor & \\
\hline Cash Cost, & \\
\hline Casting & \\
\hline Liquid Steel & $\$ 113.57$ \\
\hline
\end{tabular}

\section{R\&M Supplies}

Labor

\section{Cash Cost Slabs, FOB Plant}

\section{Debt Service, $270,000,000$ @ 10\% for 10 years}

Operating Cost / tonne steel slab

\section{Operating Cost / ton steel slab (2000 lbs)}

Year

\begin{tabular}{|c|c|c|c|}
\hline $\begin{array}{l}\text { \$/tonne } \\
\text { Source }\end{array}$ & $\begin{array}{l}\text { tonnes/ } \\
\text { tonne } \\
\text { Product }\end{array}$ & $\begin{array}{l}\text { \$/tonne } \\
\text { Product }\end{array}$ & $\begin{array}{c}\text { Total Cash } \\
\text { Cost } \\
\text { \$/tonne }\end{array}$ \\
\hline$\$ 69.36$ & 0.83 & $\$ 57.57$ & \\
\hline \multirow[t]{9}{*}{$\$ 100.00$} & 0.21 & $\$ 21.00$ & \\
\hline & & $\$ 15.00$ & \\
\hline & & $\$ 3.00$ & \\
\hline & & $\$ 3.00$ & \\
\hline & & $\$ 2.50$ & \\
\hline & & $\$ 8.50$ & \\
\hline & & $\$ 3.00$ & \\
\hline & & & $\$ 113.57$ \\
\hline & & & \\
\hline \multirow[t]{11}{*}{$\$ 113.57$} & 1.03 & $\$ 116.98$ & \\
\hline & & $\$ 2.00$ & \\
\hline & & $\$ 8.00$ & \\
\hline & & $\$ 2.50$ & \\
\hline & & & $\$ 129.48$ \\
\hline & & & $\$ 73.85$ \\
\hline & & & $\$ 203.32$ \\
\hline & & & \\
\hline & & & $\$ 184.42$ \\
\hline & & & \\
\hline & & & 585,000 \\
\hline
\end{tabular}

\section{Capital Costs}

\begin{tabular}{|l|r|}
\hline Hot Metal & $\$ 150,000,000$ \\
\hline Conversion & $\$ 70,000,000$ \\
\hline Casting & $\$ 50,000,000$ \\
\hline Total & $\$ 270,000,000$ \\
\hline
\end{tabular}

Debt Service / Year

$10 \%$ over 10 Years.

\begin{tabular}{|l|r|}
\hline Hot Metal & $\$ 24,000,000$ \\
\hline Conversion & $\$ 11,200,000$ \\
\hline Casting & $\$ 8,000,000$ \\
\hline Total & $\$ 43,200,000$ \\
\hline
\end{tabular}

Debt Service / tonne Steel $10 \%$ over 10 Years

\begin{tabular}{|l|r|}
\hline Hot Metal & $\$ 41.03$ \\
\hline Conversion & $\$ 19.15$ \\
\hline Casting & $\$ 13.68$ \\
\hline Total & $\$ 73.85$ \\
\hline
\end{tabular}

\section{Capital}

Costs per Annual Tonne

\begin{tabular}{|l|r|}
\hline Hot Metal* $^{*}$ & $\$ 300.00$ \\
\hline Conversion $^{\star \star}$ & $\$ 119.66$ \\
\hline Casting $^{\star *}$ & $\$ 85.47$ \\
\hline Total $^{\star *}$ & $\$ 461.54$ \\
\hline
\end{tabular}

* 500,000 t/a hot metal basis

** 585,000 t/a steel basis 


\section{APPENDIX D: Figure 6.1. Material Flows in Microwave Steel Making}

Hot Metal Operations:

\section{Iron Ore}

1.35

Coal

0.32

Electricity

667,233
Tonnes concentrate per tonne metal

Tonnes coal per tonne metal

Kwhr per tonne metal

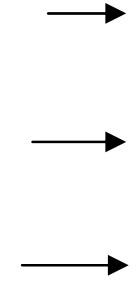

Million Tonnes

per year

333,617

$\sqrt{ }$

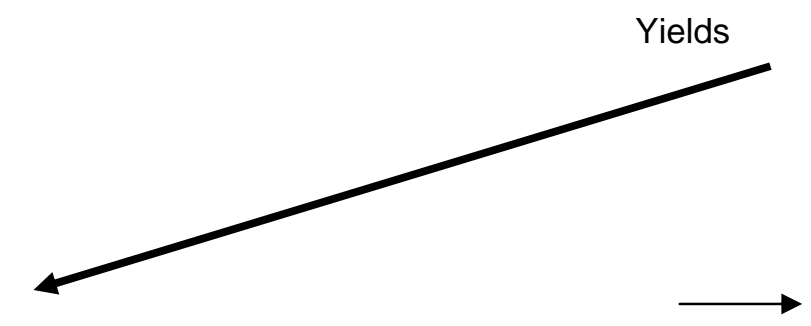

Tonnes hot metal per tonne steel

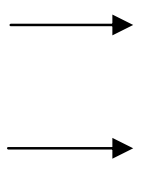

Tonnes scrap per tonne steel

$\sqrt{l}$
0.675

0.160

0.500

0.127

Tonnes Iron Ore

Tonnes Coal

Kwhr Electricity

Tonnes Hot

Metal

Produced per

year

Tonnes Hot

Metal

Tonnes Scrap

Metal

0.602

Tonnes Steel

Produced per

year

Tonnes Steel

Slabs

Produced per

year 
APPENDIX D: Table D.3A: Parameters and assumptions for producing hot metal:

\begin{tabular}{|c|c|}
\hline Parameters \& Assumptions: & Includes Inflation using $\$ 40 /$ tonne iron ore \\
\hline Hot metal production rate $=$ & 0.5 \\
\hline Steel product selling price $=$ & $\$ 300.00$ \\
\hline Royalty Rate $=$ & $1.0 \%$ \\
\hline Steel price escallation rate $=$ & $1.0 \%$ \\
\hline Cost inflation rate $=$ & $2.0 \%$ \\
\hline Steel used per tonne steel slabs produced $=$ & 0 \\
\hline Hot metal used per tonne steel $=$ & 0 \\
\hline Iron ore used per tonne hot metal = & 1.35 \\
\hline Coal used per tonne hot metal $=$ & 0.32 \\
\hline Iron ore cost per tonne $=$ & $\$ 40.00$ \\
\hline Electricity use per tonne hot metal $=$ & 667 \\
\hline Electricity cost per kwhr $=$ & $\$ 0.035$ \\
\hline Coal price per tonne $=$ & $\$ 20.00$ \\
\hline Labor wage rate $=$ & $\$ 25.00$ \\
\hline Employee overhead rate $=$ & $55 \%$ \\
\hline \multicolumn{2}{|l|}{ HOT METAL OPERATIONS: } \\
\hline Hot metal workers per year $=$ & 25 \\
\hline Salary cost per labor cost $=$ & $\$ 0.38$ \\
\hline $\mathrm{R} \& \mathrm{M}$ per tonne hot metal $=$ & $\$ 2.00$ \\
\hline General \& office expense $=$ & $\$ 1.50$ \\
\hline Capital cost per tonne hot metal $=$ & $\$ 300$ \\
\hline Proportion of capital for structures $=$ & $25 \%$ \\
\hline Property Tax Rate $=$ & $1.0 \%$ \\
\hline State Income Tax Rate $=$ & $6.0 \%$ \\
\hline Federal Income Tax Rate $=$ & $35.0 \%$ \\
\hline Capital Gain Tax Rate $=$ & $20.0 \%$ \\
\hline Equipment Salvage Value $\%=$ & $10.0 \%$ \\
\hline Building Salvage Value $\%=$ & $25.0 \%$ \\
\hline Working Capital \% of Total Capital = & $20.0 \%$ \\
\hline Cost of Capital: MARR $=$ & $10.0 \%$ \\
\hline
\end{tabular}


APPENDIX D: Table D.3B: Parameters and assumptions for producing steel slabs:

\begin{tabular}{|c|c|}
\hline Parameters \& Assumptions & \\
\hline Hot metal production rate $=$ & 0.5 \\
\hline Steel product selling price $=$ & $\$ 400.00$ \\
\hline Royalty Rate $=$ & $1.0 \%$ \\
\hline Steel price escallation rate $=$ & $1.0 \%$ \\
\hline Cost inflation rate $=$ & $2.0 \%$ \\
\hline Steel used per tonne steel slabs produced $=$ & 1.03 \\
\hline Hot metal used per tonne steel $=$ & 0.83 \\
\hline Iron ore used per tonne hot metal $=$ & 1.35 \\
\hline Coal used per tonne hot metal $=$ & 0.32 \\
\hline Iron ore cost per tonne $=$ & $\$ 40.00$ \\
\hline Electricity use per tonne hot metal $=$ & 667 \\
\hline Electricity cost per kwhr $=$ & $\$ 0.035$ \\
\hline Coal price per tonne $=$ & $\$ 20.00$ \\
\hline Labor wage rate $=$ & $\$ 25.00$ \\
\hline Employee overhead rate $=$ & $55 \%$ \\
\hline \multicolumn{2}{|l|}{ HOT METAL OPERATIONS: } \\
\hline Hot metal workers per year $=$ & 25 \\
\hline Salary cost per labor cost $=$ & $\$ 0.38$ \\
\hline $\mathrm{R} \& \mathrm{M}$ per tonne hot metal $=$ & $\$ 2.00$ \\
\hline General \& office expense $=$ & $\$ 1.50$ \\
\hline Capital cost per tonne hot metal $=$ & $\$ 300$ \\
\hline Proportion of capital for structures $=$ & $25 \%$ \\
\hline \multicolumn{2}{|l|}{ STEEL MAKING OPERATIONS: } \\
\hline Scrap used per tonne steel $=$ & 0.21 \\
\hline \multirow[b]{2}{*}{ Alloy cost per tonne steel = } & $\$ 116.00$ \\
\hline & $\$ 15.00$ \\
\hline Oxygen used per tonne steel $=$ & $\$ 3.00$ \\
\hline Flux used per tonne steel $=$ & $\$ 3.00$ \\
\hline Energy cost per tonne steel $=$ & $\$ 2.50$ \\
\hline Steel making workers per year $=$ & 22 \\
\hline Salary cost per labor cost $=$ & $\$ 0.38$ \\
\hline $\mathrm{R} \& \mathrm{M}$ per tonne steel $=$ & $\$ 8.50$ \\
\hline General \& office expense $=$ & $\$ 1.00$ \\
\hline Capital cost per tonne steel $=$ & $\$ 120$ \\
\hline Proportion of capital for structures $=$ & $25 \%$ \\
\hline \multicolumn{2}{|l|}{ STEEL CASTING OPERATIONS: } \\
\hline Energy cost per tonne of slabs produced $=$ & $\$ 2.00$ \\
\hline Casting workers per year $=$ & 13 \\
\hline Salary cost per labor cost $=$ & $\$ 0.38$ \\
\hline
\end{tabular}




\begin{tabular}{|r|c|} 
R \& M per tonne slabs $=$ & $\$ 8.00$ \\
\hline General \& office expense $=$ & $\$ 1.00$ \\
\hline Capital cost per tonne slab $=$ & $\$ 86$ \\
\hline Proportion of capital for structures $=$ & $25 \%$ \\
\hline Property Tax Rate $=$ & $1.0 \%$ \\
\hline Federal Income Tax Rate $=$ & $6.0 \%$ \\
\hline Capital Gain Tax Rate $=$ & $35.0 \%$ \\
\hline & \\
\hline Equipment Salvage Value $\%=$ & $20.0 \%$ \\
\hline Building Salvage Value $\%=$ & $10.0 \%$ \\
\hline Working Capital $\%$ of Total Capital $=$ & $25.0 \%$ \\
\hline & $20.0 \%$ \\
\hline Cost of Capital: MARR $=$ & \\
\hline
\end{tabular}


APPENDIX D: Table D.4A: Base case economic evaluation of producing hot metal:

\section{Microwave Steel as Feed for Mini-Mill: From Iron Ore to Hot Metal}

Includes Inflation using \$40/tonne iron ore

\begin{tabular}{|c|c|c|c|c|c|c|c|c|c|c|c|}
\hline & 0 & 1 & $?$ & $?$ & & & & 7 & 0 & 0 & 10 \\
\hline $\begin{array}{r}\text { YEAR } \\
\end{array}$ & $\mathbf{U}$ & $\perp$ & 2 & 5 & 4 & 5 & $\mathbf{0}$ & 7 & $\mathbf{0}$ & $\mathbf{y}$ & 10 \\
\hline \multicolumn{12}{|l|}{ REVENUES: } \\
\hline \multicolumn{12}{|l|}{ PRODUCE STEEL SLABS } \\
\hline Production, Mill. tonne/yr. & & 0.500 & 0.500 & 0.500 & 0.500 & 0.500 & 0.500 & 0.500 & 0.500 & 0.500 & 0.500 \\
\hline Price, \$/unit & & $\$ 300.0$ & $\$ 300.0$ & $\$ 300.0$ & $\$ 300.0$ & $\$ 300.0$ & $\$ 300.0$ & $\$ 300.0$ & $\$ 300.0$ & $\$ 300.0$ & $\$ 300.0$ \\
\hline Revenue \& Inflation & & $\$ 150.0$ & $\$ 153.0$ & $\$ 154.5$ & $\$ 156.1$ & $\$ 157.7$ & $\$ 159.2$ & $\$ 160.8$ & $\$ 162.4$ & $\$ 164.1$ & $\$ 165.7$ \\
\hline Royalty Payments & & $-\$ 1.5$ & $-\$ 1.5$ & $-\$ 1.5$ & $-\$ 1.6$ & $-\$ 1.6$ & $-\$ 1.6$ & $-\$ 1.6$ & $-\$ 1.6$ & $-\$ 1.6$ & $-\$ 1.7$ \\
\hline Net Revenue & & $\$ 148.5$ & $\$ 151.5$ & $\$ 153.0$ & $\$ 154.5$ & $\$ 156.1$ & $\$ 157.6$ & $\$ 159.2$ & $\$ 160.8$ & $\$ 162.4$ & $\$ 164.0$ \\
\hline \multicolumn{12}{|l|}{ COSTS: } \\
\hline \multicolumn{12}{|l|}{ IRON ORE TO HOT METAL: } \\
\hline Iron Ore & & $-\$ 27.0$ & $-\$ 27.0$ & $-\$ 27.0$ & $-\$ 27.0$ & $-\$ 27.0$ & $-\$ 27.0$ & $-\$ 27.0$ & $-\$ 27.0$ & $-\$ 27.0$ & $-\$ 27.0$ \\
\hline Electricity & & $-\$ 11.7$ & $-\$ 11.7$ & $-\$ 11.7$ & $-\$ 11.7$ & $-\$ 11.7$ & $-\$ 11.7$ & $-\$ 11.7$ & $-\$ 11.7$ & $-\$ 11.7$ & $-\$ 11.7$ \\
\hline Coal & & $-\$ 3.2$ & $-\$ 3.2$ & $-\$ 3.2$ & $-\$ 3.2$ & $-\$ 3.2$ & $-\$ 3.2$ & $-\$ 3.2$ & $-\$ 3.2$ & $-\$ 3.2$ & $-\$ 3.2$ \\
\hline Labor & & $-\$ 1.3$ & $-\$ 1.3$ & $-\$ 1.3$ & $-\$ 1.3$ & $-\$ 1.3$ & $-\$ 1.3$ & $-\$ 1.3$ & $-\$ 1.3$ & $-\$ 1.3$ & $-\$ 1.3$ \\
\hline Salaries & & $-\$ 0.5$ & $-\$ 0.5$ & $-\$ 0.5$ & $-\$ 0.5$ & $-\$ 0.5$ & $-\$ 0.5$ & $-\$ 0.5$ & $-\$ 0.5$ & $-\$ 0.5$ & $-\$ 0.5$ \\
\hline Overhead & & $-\$ 1.0$ & $-\$ 1.0$ & $-\$ 1.0$ & $-\$ 1.0$ & $-\$ 1.0$ & $-\$ 1.0$ & $-\$ 1.0$ & $-\$ 1.0$ & $-\$ 1.0$ & $-\$ 1.0$ \\
\hline R \& M Supplies & & $-\$ 1.0$ & $-\$ 1.0$ & $-\$ 1.0$ & $-\$ 1.0$ & $-\$ 1.0$ & $-\$ 1.0$ & $-\$ 1.0$ & $-\$ 1.0$ & $-\$ 1.0$ & $-\$ 1.0$ \\
\hline Lab, insurance, office & & $-\$ 1.5$ & $-\$ 1.5$ & $-\$ 1.5$ & $-\$ 1.5$ & $-\$ 1.5$ & $-\$ 1.5$ & $-\$ 1.5$ & $-\$ 1.5$ & $-\$ 1.5$ & $-\$ 1.5$ \\
\hline TOTAL CASH COST & & $-\$ 47.2$ & $-\$ 47.2$ & $-\$ 47.2$ & $-\$ 47.2$ & $-\$ 47.2$ & $-\$ 47.2$ & $-\$ 47.2$ & $-\$ 47.2$ & $-\$ 47.2$ & $-\$ 47.2$ \\
\hline INFLATED CASH COST & & $-\$ 47.2$ & $-\$ 49.1$ & $-\$ 50.0$ & $-\$ 51.0$ & $-\$ 52.1$ & $-\$ 53.1$ & $-\$ 54.2$ & $-\$ 55.2$ & $-\$ 56.4$ & $-\$ 57.5$ \\
\hline Equipment Depreciation & & $-\$ 16.1$ & $-\$ 27.6$ & $-\$ 19.7$ & $-\$ 14.1$ & $-\$ 10.0$ & $-\$ 10.0$ & $-\$ 10.0$ & $-\$ 5.0$ & $\$ 0.0$ & $\$ 0.0$ \\
\hline Building Depreciation & & $-\$ 0.9$ & $-\$ 1.0$ & $-\$ 1.0$ & $-\$ 1.0$ & $-\$ 1.0$ & $-\$ 1.0$ & $-\$ 1.0$ & $-\$ 1.0$ & $-\$ 1.0$ & $-\$ 1.0$ \\
\hline TOTAL HOT METAL COST & & $-\$ 64.1$ & $-\$ 77.6$ & $-\$ 70.7$ & $-\$ 66.1$ & $-\$ 63.1$ & $-\$ 64.1$ & $-\$ 65.2$ & $-\$ 61.2$ & $-\$ 57.3$ & $-\$ 58.4$ \\
\hline Total Cost: Hot Metal Only & & $-\$ 64$ & $-\$ 78$ & $-\$ 71$ & $-\$ 66$ & $-\$ 63$ & $-\$ 64$ & $-\$ 65$ & $-\$ 61$ & $-\$ 57$ & $-\$ 58$ \\
\hline Income before Taxes & 0 & $\$ 84.4$ & $\$ 73.9$ & $\$ 82.3$ & $\$ 88.5$ & $\$ 93.0$ & $\$ 93.5$ & $\$ 94.0$ & $\$ 99.6$ & $\$ 105.1$ & $\$ 105.6$ \\
\hline Property Taxes & 0 & $-\$ 1.5$ & $-\$ 1.5$ & $-\$ 1.5$ & $-\$ 1.5$ & $-\$ 1.5$ & $-\$ 1.5$ & $-\$ 1.5$ & $-\$ 1.5$ & $-\$ 1.5$ & $-\$ 1.5$ \\
\hline
\end{tabular}




\begin{tabular}{|c|c|c|c|c|c|c|c|c|c|c|c|}
\hline State Income Tax & 0 & $-\$ 5.0$ & $-\$ 4.3$ & $-\$ 4.8$ & $-\$ 5.2$ & $-\$ 5.5$ & $-\$ 5.5$ & $-\$ 5.6$ & $-\$ 5.9$ & $-\$ 6.2$ & $-\$ 6.2$ \\
\hline Federal Income Tax & 0 & $-\$ 27.3$ & $-\$ 23.8$ & $-\$ 26.6$ & $-\$ 28.6$ & $-\$ 30.1$ & $-\$ 30.3$ & $-\$ 30.4$ & $-\$ 32.3$ & $-\$ 34.1$ & $-\$ 34.2$ \\
\hline & & & & & & & & & & & \\
\hline After Tax Income & 0 & $\$ 50.6$ & $\$ 44.2$ & $\$ 49.4$ & $\$ 53.1$ & $\$ 55.9$ & $\$ 56.2$ & $\$ 56.5$ & $\$ 59.9$ & $\$ 63.3$ & $\$ 63.6$ \\
\hline Non-cash Charges & 0 & $\$ 17.0$ & $\$ 28.5$ & $\$ 20.6$ & $\$ 15.0$ & $\$ 11.0$ & $\$ 11.0$ & $\$ 11.0$ & $\$ 6.0$ & $\$ 1.0$ & $\$ 1.0$ \\
\hline \multicolumn{12}{|l|}{ Capital Investment } \\
\hline Hot Metal Structures & $-\$ 38$ & & & & & & & & & & $\$ 13.1$ \\
\hline Steel Making Equipment & $\$ 0$ & & & & & & & & & & $\$ 0.0$ \\
\hline Steel Making Structures & $\$ 0$ & & & & & & & & & & $\$ 0.0$ \\
\hline Casting Equipment & $\$ 0$ & & & & & & & & & & $\$ 0.0$ \\
\hline Casting Structures & $\$ 0$ & & & & & & & & & & $\$ 0.0$ \\
\hline Working Capital & & $-\$ 15.0$ & $-\$ 15.0$ & & & & & & & & $\$ 30.0$ \\
\hline
\end{tabular}

Net Present Value $=\quad \$ 262$ million $\quad$ (assumes a 10 year life)

Total Cash Costs including

royalties, taxes \& inflation

\begin{tabular}{|l|r|r|r|r|r|r|r|r|r|r|}
\hline & $-\$ 99$ & $-\$ 31$ & $-\$ 34$ & $-\$ 37$ & $-\$ 39$ & $-\$ 39$ & $-\$ 39$ & $-\$ 41$ & $-\$ 43$ & $-\$ 44$ \\
\hline
\end{tabular}

Total

per year

-\$288 Per tonne Per short ton

$\begin{array}{clll}\text { Present Worth of Cash Costs }= & -\$ 288 & \text { Per tonne } & \text { Per short } \\ \text { Present Worth of Capital Costs }= & -\$ 150 & \text { per year } & \text { per year }\end{array}$

Annualized "operating" costs =

$-\$ 47$

(1)

Annualized capital cost $=$

$-\$ 94$

per year

Total annual cost =

$-\$ 70$

$\frac{-\$ 46}{-\$ 140}$

$-\$ 85$

$-\$ 42$

$-\$ 127$ 
APPENDIX D: Table D.4B: Base case economic evaluation of producing steel slabs:

\section{Stand Alone Plant: From Iron Ore to Cast Steel Slabs}

\begin{tabular}{|c|c|c|c|c|c|c|c|c|c|c|c|}
\hline \multicolumn{12}{|c|}{ Amounts in Millions (Display is rounded to nearest $\$ 0.1$ million) } \\
\hline YEAR & 0 & 1 & 2 & 3 & 4 & 5 & 6 & 7 & 8 & 9 & 10 \\
\hline \multicolumn{12}{|l|}{ REVENUES: } \\
\hline \multicolumn{12}{|l|}{ PRODUCE STEEL SLABS } \\
\hline Production, Mill. tonne/yr. & & 0.585 & 0.585 & 0.585 & 0.585 & 0.585 & 0.585 & 0.585 & 0.585 & 0.585 & 0.585 \\
\hline Price, \$/unit & & $\$ 400.0$ & $\$ 400.0$ & $\$ 400.0$ & $\$ 400.0$ & $\$ 400.0$ & $\$ 400.0$ & $\$ 400.0$ & $\$ 400.0$ & $\$ 400.0$ & $\$ 400.0$ \\
\hline Revenue \& Inflation & & $\$ 233.9$ & $\$ 238.6$ & $\$ 241.0$ & $\$ 243.4$ & $\$ 245.9$ & $\$ 248.3$ & $\$ 250.8$ & $\$ 253.3$ & $\$ 255.9$ & $\$ 258.4$ \\
\hline Royalty Payments & & $-\$ 2.3$ & $-\$ 2.4$ & $-\$ 2.4$ & $-\$ 2.4$ & $-\$ 2.5$ & $-\$ 2.5$ & $-\$ 2.5$ & $-\$ 2.5$ & $-\$ 2.6$ & $-\$ 2.6$ \\
\hline Net Revenue & & $\$ 231.6$ & $\$ 236.3$ & $\$ 238.6$ & $\$ 241.0$ & $\$ 243.4$ & $\$ 245.9$ & $\$ 248.3$ & $\$ 250.8$ & $\$ 253.3$ & $\$ 255.8$ \\
\hline \multicolumn{12}{|l|}{ COSTS: } \\
\hline \multicolumn{12}{|l|}{ IRON ORE TO HOT METAL: } \\
\hline Iron Ore & & $-\$ 27.0$ & $-\$ 27.0$ & $-\$ 27.0$ & $-\$ 27.0$ & $-\$ 27.0$ & $-\$ 27.0$ & $-\$ 27.0$ & $-\$ 27.0$ & $-\$ 27.0$ & $-\$ 27.0$ \\
\hline Electricity & & $-\$ 11.7$ & $-\$ 11.7$ & $-\$ 11.7$ & $-\$ 11.7$ & $-\$ 11.7$ & $-\$ 11.7$ & $-\$ 11.7$ & $-\$ 11.7$ & $-\$ 11.7$ & $-\$ 11.7$ \\
\hline Coal & & $-\$ 3.2$ & $-\$ 3.2$ & $-\$ 3.2$ & $-\$ 3.2$ & $-\$ 3.2$ & $-\$ 3.2$ & $-\$ 3.2$ & $-\$ 3.2$ & $-\$ 3.2$ & $-\$ 3.2$ \\
\hline Labor & & $-\$ 1.3$ & $-\$ 1.3$ & $-\$ 1.3$ & $-\$ 1.3$ & $-\$ 1.3$ & $-\$ 1.3$ & $-\$ 1.3$ & $-\$ 1.3$ & $-\$ 1.3$ & $-\$ 1.3$ \\
\hline Salaries & & $-\$ 0.5$ & $-\$ 0.5$ & $-\$ 0.5$ & $-\$ 0.5$ & $-\$ 0.5$ & $-\$ 0.5$ & $-\$ 0.5$ & $-\$ 0.5$ & $-\$ 0.5$ & $-\$ 0.5$ \\
\hline Overhead & & $-\$ 1.0$ & $-\$ 1.0$ & $-\$ 1.0$ & $-\$ 1.0$ & $-\$ 1.0$ & $-\$ 1.0$ & $-\$ 1.0$ & $-\$ 1.0$ & $-\$ 1.0$ & $-\$ 1.0$ \\
\hline R \& M Supplies & & $-\$ 1.0$ & $-\$ 1.0$ & $-\$ 1.0$ & $-\$ 1.0$ & $-\$ 1.0$ & $-\$ 1.0$ & $-\$ 1.0$ & $-\$ 1.0$ & $-\$ 1.0$ & $-\$ 1.0$ \\
\hline Lab, insurance, office & & $-\$ 1.5$ & $-\$ 1.5$ & $-\$ 1.5$ & $-\$ 1.5$ & $-\$ 1.5$ & $-\$ 1.5$ & $-\$ 1.5$ & $-\$ 1.5$ & $-\$ 1.5$ & $-\$ 1.5$ \\
\hline TOTAL CASH COST & & $-\$ 47.2$ & $-\$ 47.2$ & $-\$ 47.2$ & $-\$ 47.2$ & $-\$ 47.2$ & $-\$ 47.2$ & $-\$ 47.2$ & $-\$ 47.2$ & $-\$ 47.2$ & $-\$ 47.2$ \\
\hline INFLATED CASH COST & & $-\$ 47.2$ & $-\$ 49.1$ & $-\$ 50.0$ & $-\$ 51.0$ & $-\$ 52.1$ & $-\$ 53.1$ & $-\$ 54.2$ & $-\$ 55.2$ & $-\$ 56.4$ & $-\$ 57.5$ \\
\hline Equipment Depreciation & & $-\$ 16.1$ & $-\$ 27.6$ & $-\$ 19.7$ & $-\$ 14.1$ & $-\$ 10.0$ & $-\$ 10.0$ & $-\$ 10.0$ & $-\$ 5.0$ & $\$ 0.0$ & $\$ 0.0$ \\
\hline Building Depreciation & & $-\$ 0.9$ & $-\$ 1.0$ & $-\$ 1.0$ & $-\$ 1.0$ & $-\$ 1.0$ & $-\$ 1.0$ & $-\$ 1.0$ & $-\$ 1.0$ & $-\$ 1.0$ & $-\$ 1.0$ \\
\hline TOTAL HOT METAL COST & & $-\$ 64.1$ & $-\$ 77.6$ & $-\$ 70.7$ & $-\$ 66.1$ & $-\$ 63.1$ & $-\$ 64.1$ & $-\$ 65.2$ & $-\$ 61.2$ & $-\$ 57.3$ & $-\$ 58.4$ \\
\hline \multicolumn{12}{|l|}{ HOT METAL TO STEEL: } \\
\hline Scrap iron & & $-\$ 14.7$ & $-\$ 14.7$ & $-\$ 14.7$ & $-\$ 14.7$ & $-\$ 14.7$ & $-\$ 14.7$ & $-\$ 14.7$ & $-\$ 14.7$ & $-\$ 14.7$ & $-\$ 14.7$ \\
\hline Alloys & & $-\$ 9.0$ & $-\$ 9.0$ & $-\$ 9.0$ & $-\$ 9.0$ & $-\$ 9.0$ & $-\$ 9.0$ & $-\$ 9.0$ & $-\$ 9.0$ & $-\$ 9.0$ & $-\$ 9.0$ \\
\hline Oxygen & & $-\$ 1.8$ & $-\$ 1.8$ & $-\$ 1.8$ & $-\$ 1.8$ & $-\$ 1.8$ & $-\$ 1.8$ & $-\$ 1.8$ & $-\$ 1.8$ & $-\$ 1.8$ & $-\$ 1.8$ \\
\hline
\end{tabular}




\begin{tabular}{|c|c|c|c|c|c|c|c|c|c|c|c|}
\hline Flux & & $-\$ 1.8$ & $-\$ 1.8$ & $-\$ 1.8$ & $-\$ 1.8$ & $-\$ 1.8$ & $-\$ 1.8$ & $-\$ 1.8$ & $-\$ 1.8$ & $-\$ 1.8$ & $-\$ 1.8$ \\
\hline Energy & & $-\$ 1.5$ & $-\$ 1.5$ & $-\$ 1.5$ & $-\$ 1.5$ & $-\$ 1.5$ & $-\$ 1.5$ & $-\$ 1.5$ & $-\$ 1.5$ & $-\$ 1.5$ & $-\$ 1.5$ \\
\hline Labor & & $-\$ 1.1$ & $-\$ 1.1$ & $-\$ 1.1$ & $-\$ 1.1$ & $-\$ 1.1$ & $-\$ 1.1$ & $-\$ 1.1$ & $-\$ 1.1$ & $-\$ 1.1$ & $-\$ 1.1$ \\
\hline Salaries & & $-\$ 0.4$ & $-\$ 0.4$ & $-\$ 0.4$ & $-\$ 0.4$ & $-\$ 0.4$ & $-\$ 0.4$ & $-\$ 0.4$ & $-\$ 0.4$ & $-\$ 0.4$ & $-\$ 0.4$ \\
\hline Overhead & & $-\$ 0.9$ & $-\$ 0.9$ & $-\$ 0.9$ & $-\$ 0.9$ & $-\$ 0.9$ & $-\$ 0.9$ & $-\$ 0.9$ & $-\$ 0.9$ & $-\$ 0.9$ & $-\$ 0.9$ \\
\hline R \& M Supplies & & $-\$ 5.1$ & $-\$ 5.1$ & $-\$ 5.1$ & $-\$ 5.1$ & $-\$ 5.1$ & $-\$ 5.1$ & $-\$ 5.1$ & $-\$ 5.1$ & $-\$ 5.1$ & $-\$ 5.1$ \\
\hline Lab, insurance, office & & $-\$ 1.0$ & $-\$ 1.0$ & $-\$ 1.0$ & $-\$ 1.0$ & $-\$ 1.0$ & $-\$ 1.0$ & $-\$ 1.0$ & $-\$ 1.0$ & $-\$ 1.0$ & $-\$ 1.0$ \\
\hline TOTAL CASH COST & & $-\$ 37.4$ & $-\$ 37.4$ & $-\$ 37.4$ & $-\$ 37.4$ & $-\$ 37.4$ & $-\$ 37.4$ & $-\$ 37.4$ & $-\$ 37.4$ & $-\$ 37.4$ & $-\$ 37.4$ \\
\hline INFLATED CASH COST & & $-\$ 37.4$ & $-\$ 38.9$ & $-\$ 39.7$ & $-\$ 40.5$ & $-\$ 41.3$ & $-\$ 42.1$ & $-\$ 43.0$ & $-\$ 43.8$ & $-\$ 44.7$ & $-\$ 45.6$ \\
\hline Equipment Depreciation & & $-\$ 7.7$ & $-\$ 13.3$ & $-\$ 9.5$ & $-\$ 6.8$ & $-\$ 4.8$ & $-\$ 4.8$ & $-\$ 4.8$ & $-\$ 2.4$ & $\$ 0.0$ & $\$ 0.0$ \\
\hline Building Depreciation & & $-\$ 0.4$ & $-\$ 0.5$ & $-\$ 0.5$ & $-\$ 0.5$ & $-\$ 0.5$ & $-\$ 0.5$ & $-\$ 0.5$ & $-\$ 0.5$ & $-\$ 0.5$ & $-\$ 0.5$ \\
\hline TOTAL STEEL COST & & $-\$ 45.6$ & $-\$ 52.7$ & $-\$ 49.6$ & $-\$ 47.7$ & $-\$ 46.6$ & $-\$ 47.4$ & $-\$ 48.3$ & $-\$ 46.7$ & $-\$ 45.2$ & $-\$ 46.1$ \\
\hline \multicolumn{12}{|l|}{ CASTING STEEL INTO SLABS: } \\
\hline Energy & & $-\$ 1.2$ & $-\$ 1.2$ & $-\$ 1.2$ & $-\$ 1.2$ & $-\$ 1.2$ & $-\$ 1.2$ & $-\$ 1.2$ & $-\$ 1.2$ & $-\$ 1.2$ & $-\$ 1.2$ \\
\hline Labor & & $-\$ 0.7$ & $-\$ 0.7$ & $-\$ 0.7$ & $-\$ 0.7$ & $-\$ 0.7$ & $-\$ 0.7$ & $-\$ 0.7$ & $-\$ 0.7$ & $-\$ 0.7$ & $-\$ 0.7$ \\
\hline Salaries & & $-\$ 0.3$ & $-\$ 0.3$ & $-\$ 0.3$ & $-\$ 0.3$ & $-\$ 0.3$ & $-\$ 0.3$ & $-\$ 0.3$ & $-\$ 0.3$ & $-\$ 0.3$ & $-\$ 0.3$ \\
\hline Overhead & & $-\$ 0.5$ & $-\$ 0.5$ & $-\$ 0.5$ & $-\$ 0.5$ & $-\$ 0.5$ & $-\$ 0.5$ & $-\$ 0.5$ & $-\$ 0.5$ & $-\$ 0.5$ & $-\$ 0.5$ \\
\hline R \& M Supplies & & $-\$ 0.2$ & $-\$ 0.2$ & $-\$ 0.2$ & $-\$ 0.2$ & $-\$ 0.2$ & $-\$ 0.2$ & $-\$ 0.2$ & $-\$ 0.2$ & $-\$ 0.2$ & $-\$ 0.2$ \\
\hline Lab, insurance, office & & $-\$ 0.6$ & $-\$ 0.6$ & $-\$ 0.6$ & $-\$ 0.6$ & $-\$ 0.6$ & $-\$ 0.6$ & $-\$ 0.6$ & $-\$ 0.6$ & $-\$ 0.6$ & $-\$ 0.6$ \\
\hline TOTAL CASH COST & & $-\$ 3.4$ & $-\$ 3.4$ & $-\$ 3.4$ & $-\$ 3.4$ & $-\$ 3.4$ & $-\$ 3.4$ & $-\$ 3.4$ & $-\$ 3.4$ & $-\$ 3.4$ & $-\$ 3.4$ \\
\hline INFLATED CASH COST & & $-\$ 3.4$ & $-\$ 3.6$ & $-\$ 3.6$ & $-\$ 3.7$ & $-\$ 3.8$ & $-\$ 3.9$ & $-\$ 3.9$ & $-\$ 4.0$ & $-\$ 4.1$ & $-\$ 4.2$ \\
\hline Equipment Depreciation & & $-\$ 5.4$ & $-\$ 9.2$ & $-\$ 6.6$ & $-\$ 4.7$ & $-\$ 3.4$ & $-\$ 3.4$ & $-\$ 3.4$ & $-\$ 1.7$ & $\$ 0.0$ & $\$ 0.0$ \\
\hline Building Depreciation & & $-\$ 0.3$ & $-\$ 0.3$ & $-\$ 0.3$ & $-\$ 0.3$ & $-\$ 0.3$ & $-\$ 0.3$ & $-\$ 0.3$ & $-\$ 0.3$ & $-\$ 0.3$ & $-\$ 0.3$ \\
\hline TOTAL SLAB COST & & $-\$ 9.1$ & $-\$ 13.1$ & $-\$ 10.6$ & $-\$ 8.7$ & $-\$ 7.5$ & $-\$ 7.5$ & $-\$ 7.6$ & $-\$ 6.0$ & $-\$ 4.4$ & $-\$ 4.5$ \\
\hline $\begin{array}{l}\text { Total Cost: } \\
\text { Metal+Steel+Casting }\end{array}$ & & $-\$ 119$ & $-\$ 143$ & $-\$ 131$ & $-\$ 123$ & $-\$ 117$ & $-\$ 119$ & $-\$ 121$ & $-\$ 114$ & $-\$ 107$ & $-\$ 109$ \\
\hline Income before Taxes & 0 & $\$ 112.8$ & $\$ 92.9$ & $\$ 107.8$ & $\$ 118.5$ & $\$ 126.3$ & $\$ 126.8$ & $\$ 127.3$ & $\$ 136.9$ & $\$ 146.4$ & $\$ 146.8$ \\
\hline Property Taxes & 0 & $-\$ 2.7$ & $-\$ 2.7$ & $-\$ 2.7$ & $-\$ 2.7$ & $-\$ 2.7$ & $-\$ 2.7$ & $-\$ 2.7$ & $-\$ 2.7$ & $-\$ 2.7$ & $-\$ 2.7$ \\
\hline State Income Tax & 0 & $-\$ 6.6$ & $-\$ 5.4$ & $-\$ 6.3$ & $-\$ 6.9$ & $-\$ 7.4$ & $-\$ 7.4$ & $-\$ 7.5$ & $-\$ 8.0$ & $-\$ 8.6$ & $-\$ 8.6$ \\
\hline Federal Income Tax & 0 & $-\$ 36.2$ & $-\$ 29.7$ & $-\$ 34.6$ & $-\$ 38.1$ & $-\$ 40.7$ & $-\$ 40.8$ & $-\$ 41.0$ & $-\$ 44.1$ & $-\$ 47.3$ & $-\$ 47.4$ \\
\hline Income after Tax & 0 & $\$ 67.2$ & $\$ 55.1$ & $\$ 64.2$ & $\$ 70.7$ & $\$ 75.5$ & $\$ 75.8$ & $\$ 76.1$ & $\$ 82.0$ & $\$ 87.8$ & $\$ 88.1$ \\
\hline
\end{tabular}




\begin{tabular}{|c|c|c|c|c|c|c|c|c|c|c|c|}
\hline Non-cash Charges & 0 & $\$ 30.9$ & $\$ 51.8$ & $\$ 37.5$ & $\$ 27.3$ & $\$ 20.0$ & $\$ 20.0$ & $\$ 20.0$ & $\$ 10.9$ & $\$ 1.7$ & $\$ 1.7$ \\
\hline \multicolumn{12}{|l|}{ Capital Investment } \\
\hline Hot Metal Equipment & $-\$ 113$ & & & & & & & & & & $\$ 9.0$ \\
\hline Hot Metal Structures & $-\$ 38$ & & & & & & & & & & $\$ 13.1$ \\
\hline Casting Equipment & $-\$ 38$ & & & & & & & & & & $\$ 3.0$ \\
\hline Casting Structures & $-\$ 13$ & & & & & & & & & & $\$ 4.4$ \\
\hline Working Capital & & $-\$ 27.3$ & $-\$ 27.3$ & & & & & & & & $\$ 54.5$ \\
\hline Net Cash Flow & $-\$ 273$ & $\$ 71$ & $\$ 80$ & $\$ 102$ & $\$ 98$ & $\$ 95$ & $\$ 96$ & $\$ 96$ & $\$ 93$ & $\$ 90$ & $\$ 184$ \\
\hline
\end{tabular}

Net Present Value $=$

\$316 million (assumes a 10 year life)

All non-capital costs including royalties, taxes \& inflation

Only operating costs with inflation

\begin{tabular}{|c|c|c|c|c|c|c|c|c|c|c|}
\hline & $-\$ 136$ & $-\$ 132$ & $-\$ 139$ & $-\$ 145$ & $-\$ 150$ & $-\$ 153$ & $-\$ 155$ & $-\$ 161$ & $-\$ 166$ & $-\$ 169$ \\
\hline & $-\$ 88$ & $-\$ 92$ & $-\$ 93$ & $-\$ 95$ & $-\$ 97$ & $-\$ 99$ & $-\$ 101$ & $-\$ 103$ & $-\$ 105$ & $-\$ 107$ \\
\hline
\end{tabular}

Present Worth of all non-capital Costs =

Total

Present Worth of only operating costs =

per year

$-\$ 906$

Present Worth of Capital Costs =

$-\$ 593$

$-\$ 273$

(million \$)

Annualized "operating" costs =

$-\$ 96$

\$ per tonne

\$ per ton

Annualized "operating" costs =

$-\$ 147$

$\underline{-\$ 42}$

Annualized capital cost =

$-\$ 189$

per year

$-\$ 165$

per year

$-\$ 150$

$-\$ 252$

$-\$ 229$

Total annual cost $=$

$\underline{-\$ 72}$

$\underline{-\$ 65}$

$-\$ 324$

$-\$ 294$ 